\title{
Actionable Capability for Social and Economic Systems (ACSES)
}

\section{Georgia Institute of Technology}

Peter Brecke

Patricia Voorheis

Thom McLean

\section{IntePoint, LLC}

Mark Armstrong

Oak Ridge Center for Advanced Studies

Paul Gilman

Heather O'Maonaigh

\section{Oak Ridge National Laboratory}

Steven Fernandez

Auroop Ganguly

Erin Middleton

Amy Rose

Phil Coleman

Olufemi Omitaomu

Cathy Jiao

University of North Carolina at Charlotte

Mirsad Hadzikadic

Chris Eichelberger

Theodore Carmichael

Moutaz Khouja

Joseph Rutherford

Amar Saric

Min Sun

Joseph Whitmeyer

Contract POC:

Steven Fernandez

PO Box 2008, MS 6017

Oak Ridge, TN 37831

Voice: (865) 576-3565

fernandezsj@ornl.gov

Date of Report

May 2008 


\section{TABLE OF CONTENTS}

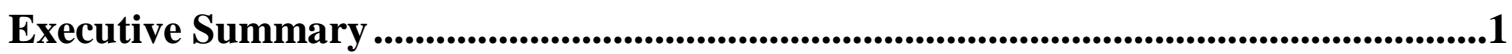

\section{Chapter 1: Evaluation of Data Availability and Worldwide Survey}

of Data Quality .......................................................................................................6

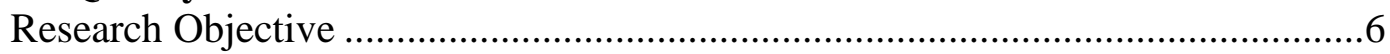

Definition of Data-quality Levels At the National Level ........................................6

Definition of Independent Variables.....................................................................

Assigning Data-Quality Levels at a Dimension Level ............................................7

Maps of Data Quality by Dimension ....................................................................

The Afghan Example ..............................................................................................13

Lessons Learned and Insights ..............................................................................17

Chapter 2: Agent-Based Modeling.......................................................................................19

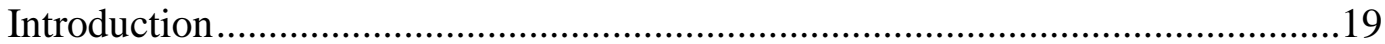

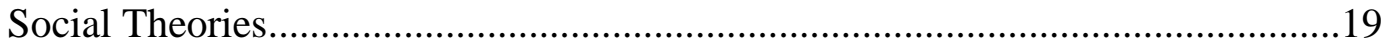

The Leadership Theories........................................................................21

The Social Mobilization Theories............................................................22

The Relationship between Social Theories..................................................23

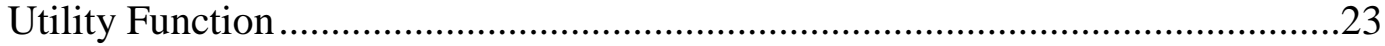

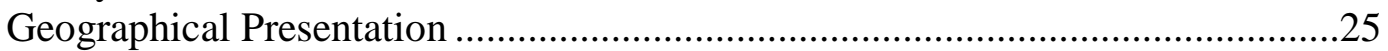

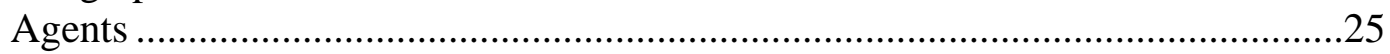

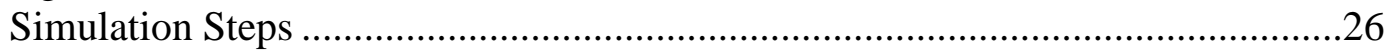

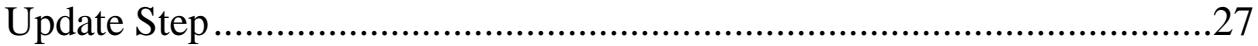

Action Step......................................................................................27

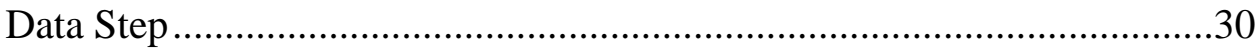

Learning Step ……….........................................................................

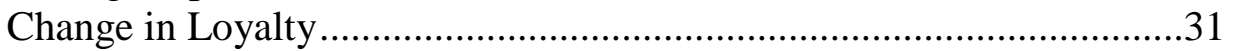

Change in Ideology ..........................................................................34

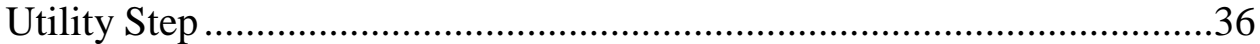

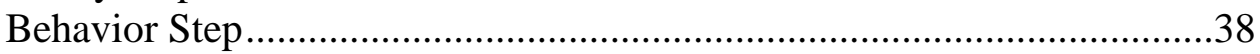

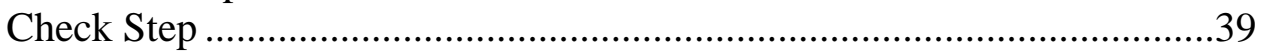

NetLogo Simulation Outputs ...............................................................................39

Territorial Control................................................................................39

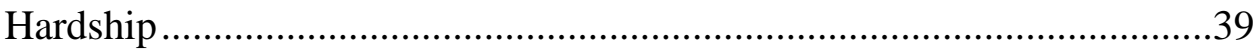

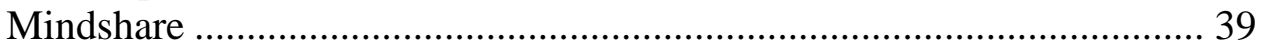

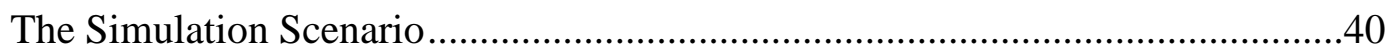

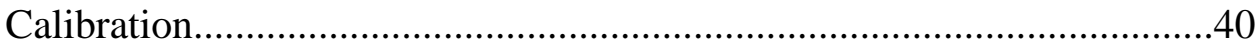

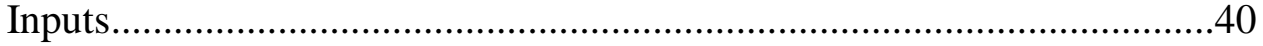

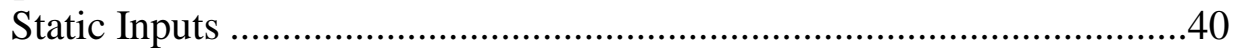

Regarding Assassination.......................................................................

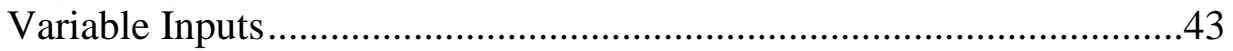

Concerning Time and Scheduling........................................................ 43

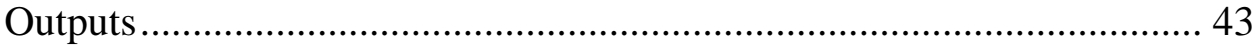


Three Incremental Agent Designs ..................................................................44

Approach 1 - Fixed Attribute Values .......................................................44

Generalizing across Parameter Settings...............................................45

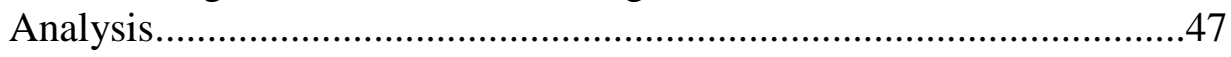

Approach 2 - Learning .................................................................... 49

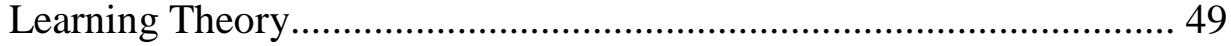

Implementation of Learning ..........................................................50

Results from Implementing Learning ................................................51

Analysis...............................................................................53

Approach 3 - Calibration via a Genetic Algorithm ................................54

Technical Design of the GA ......................................................... 54

Results from the GA …..................................................................55

Analysis of the GA.......................................................................57

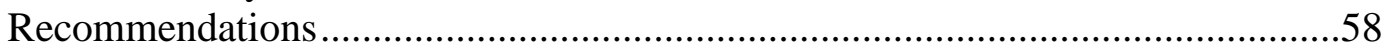

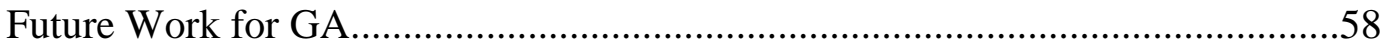

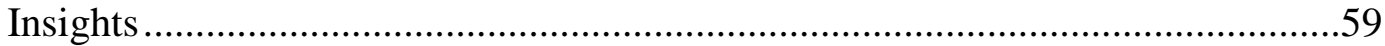

Chapter 3: Real-Time Updating ...............................................................................................60

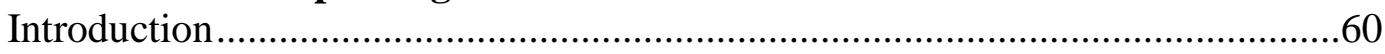

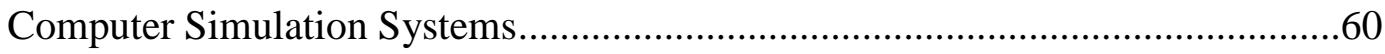

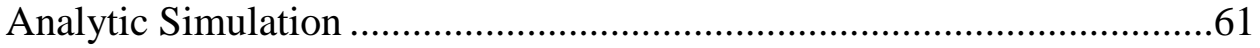

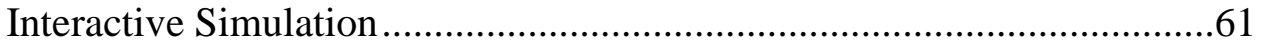

Simulation Execution Models .................................................................62

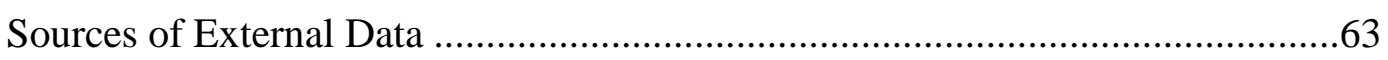

Autocoding of Free Text and Real-Time Event Generation ....................67

Anticipated Benefit from Runtime Input .........................................................68

Problem Description and Conceptual Model .....................................................69

The Synchronization Problem..............................................................69

The Mapping Problem .................................................................... 71

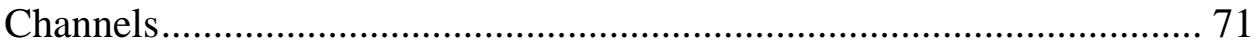

Event Handlers...................................................................................... 71

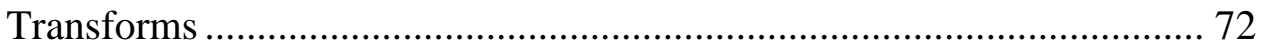

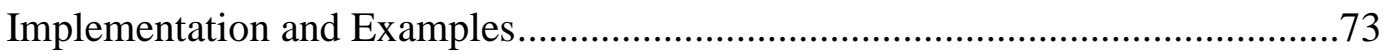

Autocoding and External Data Sources .......................................................... 75

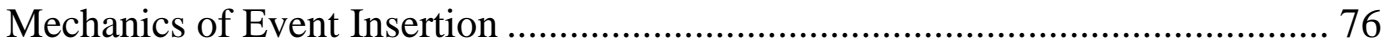

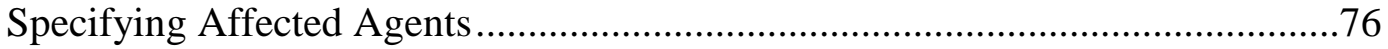

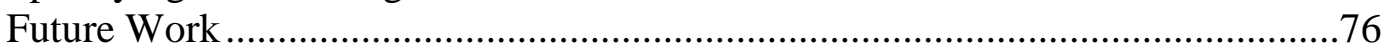

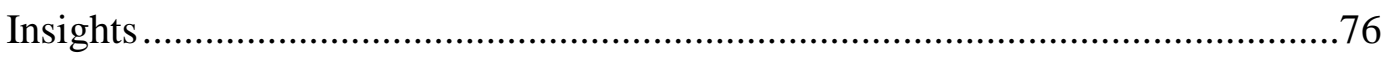

Chapter 4: Accounting for Surprise.....................................................................78

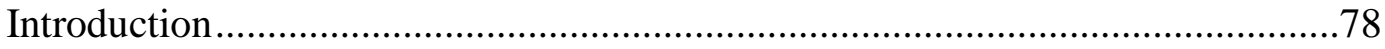

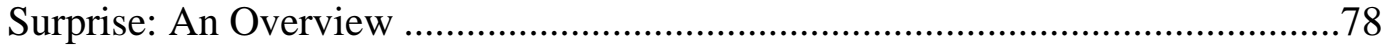

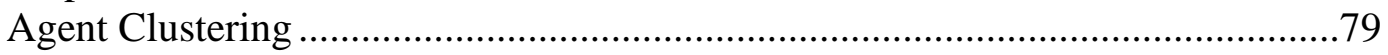

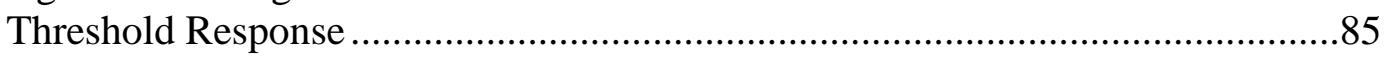

Evaluating Command Options for Ghazni in 2006.........................................88

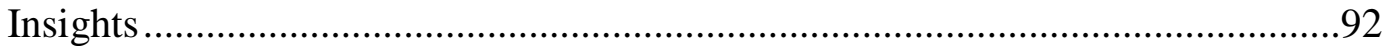


Chapter 5: Required Computational Complexity ...............................................................93

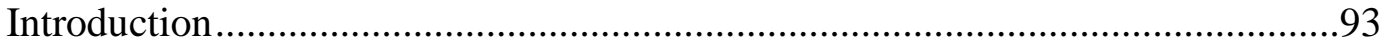

Addressing the Computational Complexity Issue..................................................95

Computational Costs ......................................................................................103

Simulation Setup of the ORMAC System ........................................................... 104

Summary of the Adaptation of the Social Theories for

the Comparison Experiment ...................................................................104

Design of the ORMAC Agent................................................................107

Geographical Attributes......................................................................107

Mandatory Agent Attributes ................................................................108

Flow Diagram of the ORMAC Simulation..............................................109

ORMAC Simulation Output Description-Feasibility Experiment.........................110

Comparison of NetLogo and ORMAC Results in Response

to the Commander's Questions .................................................................116

Insights and Conclusions .....................................................................................123

References ..............................................................................................................................125

Appendices

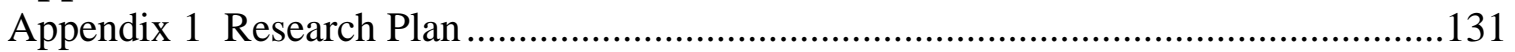

Appendix 2 Data Sources for Independent Variables.......................................................136

Appendix 3 LandScan Methodology ………………..................................................144

Appendix 4 Narrative Description of Key Data Sources................................................151

Appendix 5 Dimension Data Quality by Country............................................................166

Appendix 6 Real-World Correlation..............................................................................173

Appendix 7 Real-Time Update Code Description ...................................................177

Appendix 8 Generating Narrative.............................................................................188

Appendix 9 Visual Analytics ....................................................................................191

Appendix 10 Visual Plots of NetLogo and ORMAC Comparisons ................................197

\section{Tables}

Table 2-1 Theories implemented in the simulation model .........................................20

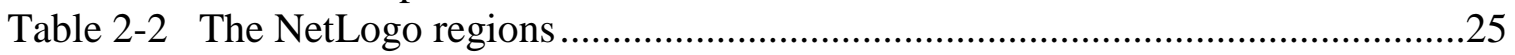

Table 2-3 Agent ability to identify other agents ............................................................27

Table 2-4 Simulation procedures within the conceptual flow diagram ...........................42

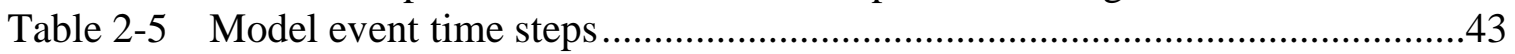

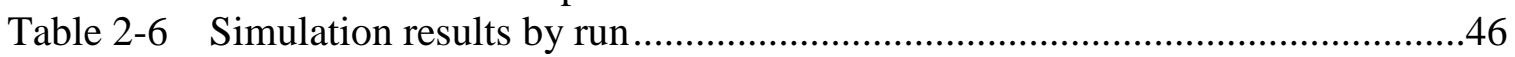

Table 2-7 Statistically significant parameter settings ........................................................46

Table 2-8 Recommended parameter settings ................................................................47

Table 2-9 The additional evaluation series ................................................................51

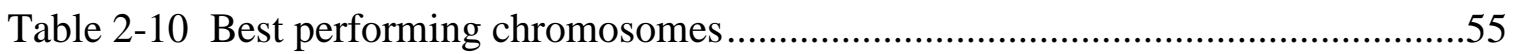

Table 3-1 Analytic and interactive execution models...................................................62

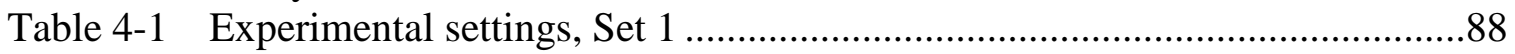

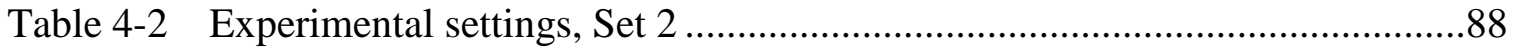

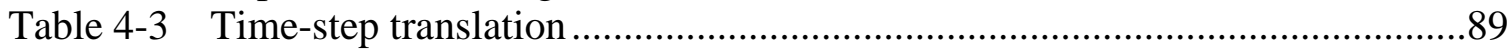

Table 5-1 Complexity comparison between ACSES and Germann simulation systems ................................................................................102

Table 5-2 Mindshare from Gallup World Poll..............................................................107 
Table 5-3 Correlation coefficients between minority opinion

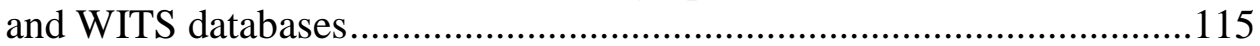

Table 5-4 Points of event insertion ..........................................................................118

Table 5-5 Correlation coefficients between NetLogo and ORMAC outputs ................119

Table 5-6 MSE between NetLogo and ORMAC outputs ...............................................120

Table 5-7 Correlation coefficients between runs for NetLogo outputs .........................120

Table 5-8 Correlation coefficients between runs for ORMAC outputs ..........................120

Table 5-9 Linear correlation between ORMAC and NetLogo runs at district levels .........................................................................122

Table A4-1 Example district profile ..............................................................................159

Table A4-2 Gallup World Poll coverage …………….................................................161

Table A4-3 Performance indices of the World Path...........................................................164

\section{Figures}

Figure 1-1 Quality of data needed to build a synthetic population ...................................9

Figure 1-2 Flow of data source employment in construction of the Afghan synthetic population.............................................................13

Figure 1-3 Geospatial distribution of agents representing the Afghan population...........14

Figure 1-4 Ethno-linguistic mappings used for ethnic interpolation ................................15

Figure 1-5 Perceived war zones ..................................................................................16

Figure 1-6 Areas of opium cultivation.......................................................................17

Figure 2-1 Conceptual flow chart of the simulation .....................................................26

Figure 2-2 Results of action choices ............................................................................30

Figure 2-3 The results of varying parameters across multiple replications ......................51

Figure 2-4 Course of simulations..................................................................................56

Figure 2-5 The difference between mindshare and control in the simulation ..................57

Figure 3-1 Logical structure of a TENA system...........................................................64

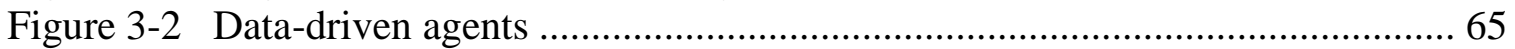

Figure 3-3 A process for updating forecasts (Plale 2005) …………………………........66

Figure 3-4 Comparing models for updating forecasts ......................................................66

Figure 3-5 Two-step transformation process .................................................................70

Figure 3-6 Notional geocoding construct ………………...........................................73

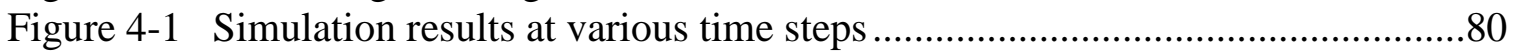

Figure 4-2 Simulation results for Runs 1-5 .............................................................82

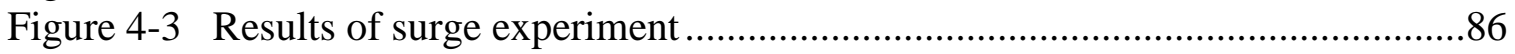

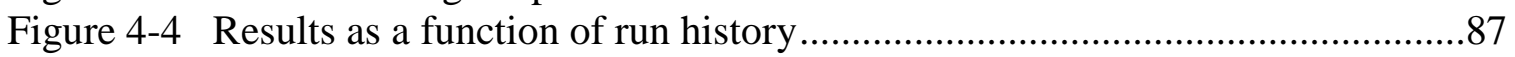

Figure 4-5 Resulting Ghazni mindshare for various parameter settings...........................89

Figure 5-1 The conceptual design of the agent ……………..........................................97

Figure 5-2 Germann model notional architecture ............................................................99

Figure 5-3 Example goal, standard, and preference tree ………………………….........100

Figure 5-4 Flow diagram of social mobilization theories plus cognitive dissonance theory .....................................................................109

Figure 5-5 Flow diagram of social mobilization theories plus homophily theory .........110

Figure 5-6 Percentage pro-Taliban minority opinion by district .......................................111

Figure 5-7 Distribution of pro-Taliban minority opinion ................................................111

Figure 5-8 Distribution of Taliban terrorist incidents.....................................................112 
Figure 5-9 Distribution of Taliban terror incidents with Ghazni

province highlighted .........................................................................113

Figure 5-10 Distribution of pro-Taliban minority opinion

under representative theory..............................................................113

Figure 5-11 Minority pro-Taliban opinion correlated with 2006 terrorist incidents ......114

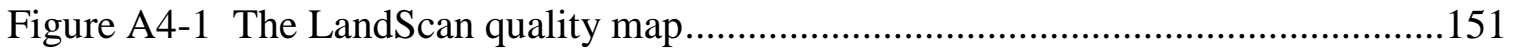

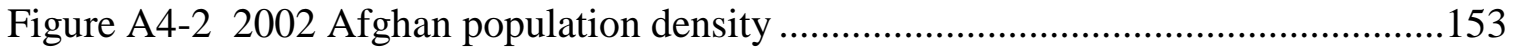

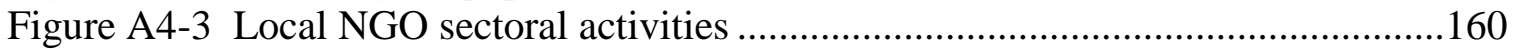

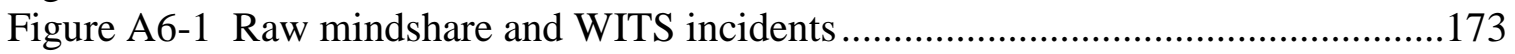

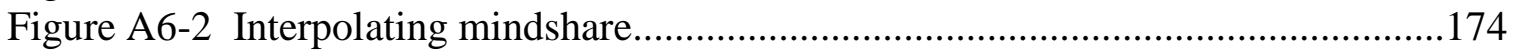

Figure A6-3 Distributing a single discreet WITS incident over 5 days.........................174

Figure A6-4 Distributing a series of 7 discreet WITS incidents over 14 days ................175

Figure A6-5 Visualization of distributed WITS data.................................................175

Figure A6-6 Correlations with assumed delay..........................................................176

Figure A9-1 Original NetLogo home page .............................................................191

Figure A9-2 Example workspace in new probe-based interface ..................................193

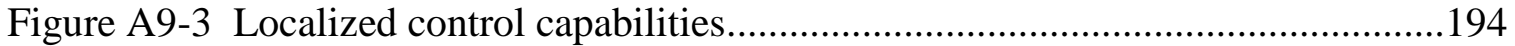

Figure A9-4 Two probes over a Taliban-controlled area.............................................195

Figure A10-1 Time-series plots of NetLogo and ORMAC simulations........................ 197 


\section{Executive Summary}

The foundation of the Actionable Capability for Social and Economic Systems (ACSES) project is a useful regional-scale social-simulation system. This report is organized into five chapters that describe insights that were gained concerning the five key feasibility questions pertaining to such a system:

1. Should such a simulation system exist, would the current state of data sets or collectable data sets be adequate to support such a system?

2. By comparing different agent-based simulation systems, is it feasible to compare simulation systems and select one appropriate for a given application with agents behaving according to modern social theory rather than ad hoc rule sets?

3. Provided that a selected simulation system for a region of interest could be constructed, can the simulation system be updated with new and changing conditions so that the universe of potential outcomes are constrained by events on the ground as they evolve?

4. As these results are constrained by evolving events on the ground, is it feasible to still generate surprise and emerging behavior to suggest outcomes from novel courses of action?

5. As these systems may for the first time require large numbers (hundreds of millions) of agents operating with complexities demanded of modern social theories, can results still be generated within actionable decision cycles?

Chapter 1 identifies and characterizes global data sources and the granularity needed to synthesize and model populations with sufficient detail to support social-simulation environments. Data describing worldwide demographic, economic, and social attributes of the world population have previously been deemed inadequate for these purposes. However, geospatial sciences have advanced a number of techniques for merging disparate databases to achieve new levels of granularity for social simulators.

A survey was conducted to determine whether the data required to build synthetic populations exist and if so, to assess data quality as it pertains to building simulationuseful synthetic populations. The suitability of indicator data sets was illustrated by building an example synthetic population for a typical case study. Afghanistan was selected for this case study because gathering data for Afghanistan supported other tasks of the ACSES project and because its data availability situation was thought to be especially challenging. Chapter 1 describes how the following disparate types of information can be integrated to construct useful synthetic populations.

- $\quad$ LandScan Global is used by a majority of key U.S. and foreign agencies as their base data system for worldwide geospatial distribution of populations. Population dynamics are available on a $1 \mathrm{sq} \mathrm{km}$ grid and can be augmented through current imagery programs. This capability is a recent contribution to the National Geospatial-Intelligence Agency's (NGA) geospatial data mission. 
- Data on military and peacekeeper forces' loyalties, readiness, and deployment are widely available through a combination of UN and classified force projections and reside mostly within classified sources.

- $\quad$ Economic data, although usually collected at the national level, can be disaggregated by applying data fusion techniques (similar to those applied to the LandScan Global databases) to data on industrial plant location, agricultural land use, and workforce distribution.

- Data sets on social attitudes, beliefs, and social cleavages have been developed for a majority of the globe (on a population basis) through anthropological studies, worldwide polling data, and classified sources. These sets can be disaggregated to the level needed to construct synthetic populations.

- $\quad$ Informational systems networks can be built to imitate civilian and propaganda channels in addition to traditional command and control networks, including communication infrastructures and transportation routing. These data sets typically require access to classified data sources.

- $\quad$ Infrastructure systems and networks have adequate modeling of engineered structures and can handle infrastructure interruption and repair from targeted and widespread disruption. Information on infrastructure systems is available in both open and classified sources.

Although direct, highly disaggregated data (at the lowest level of a population) would be difficult to collect directly, attitudes and beliefs can be credibly ascertained using two forms of disaggregation. The most common form is geographical disaggregation. In the case of modeling a population, the location of different population subgroups can be inferred from land-use patterns.

The second form of data disaggregation is category disaggregation. Data is collected and individual cases sorted with respect to different categories of a theoretically relevant variable. For example, a population can be disaggregated by ethnicity. Ethnically differentiated data such as that gleaned from opinion polls can be combined with geographically disaggregated data, as has been done by LandScan, to establish a value for local populations’ attitudes about the government or insurgent groups.

Within Chapter 2, we demonstrated that it is possible to build agent-based systems informed by modern social theory and compare the results in such a way that the relative skill of the different models can be evaluated. For this comparison we employed three theories within a case study about control and mindshare in modern Afghanistan. Critically important is the feasibility of modeling the relevant behavior of populations, beginning at the level of the individual, or agent. In Chapter 2, we explored three options for describing agent behavior in our simulation. Each approach built upon the previous one, as more adaptability was added to agent behavior. Specifically, the first approach employed agents with static attributes, fixed at the beginning of the run and unchanged 
throughout the simulation. In the second approach, agents were initially assigned attributes, but these could change over time as the agents learned and adapted to their situations. The third approach began with assignment of random attribute values, and the model was then calibrated with a genetic algorithm to favor those values that produced simulation results most predictive of the real Afghanistan. The shift over time in mindshare of Afghans, as predicted by simulation runs with each of these agent models, was compared with the real-world occurrence of terrorist events in Afghanistan. Some of the key lessons learned and insights were as follows:

1. It is possible - even within a simulation that relies heavily on stochastic processes - to evaluate multiple replications of discrete combinations of parameter values to identify optimal simulation settings and trends.

2. The simulation can exploit the underlying social models. This is a direct consequence of the equations embodying the social models, rather than an emergent behavior of the society of agents.

3. Adaptation can increase the verisimilitude of the running model in some cases.

4. The comparisons we performed suggest that the third (genetic algorithm) approach to agent behavior performed the best: it explored the most granular search space, and produced results that were entirely consistent with our earlier findings.

Chapter 3 evaluates the feasibility of injecting real-time events, and several approaches are suggested. To handle the volume of documents that could explain relevant events, documents and cables and knowledge discovery tools are crucial to allow feasible real time updating. Currently employed systems can be deployed to retrieve real-time information, de-duplicate, and screen data, and an information-extraction software component can be inserted to extract events from the data produced by Piranha. These events can be incorporated into the ACSES simulation as real-time updates.

Chapter 4 discusses the feasibility of constraining results by evolving events while still generating surprise and emerging behavior with a complex adaptive system model of roughly 10,000 agents, each of which has its own rules and attributes that take into account social, economic, and military factors at the local level. The experiments identified two distinct types of surprising, or emergent, behavior and used those results as a basis for evaluating various courses of action (COA).

Many recent advances described within Chapter 5 permit us to consider the computational requirements of the next generation of human, social, behavioral, and cultural (HSBC) modeling and simulation systems that support the ability to anticipate adverse human behavioral developments, and that allow us to evaluate alternative courses of action to mitigate their impact on U.S. national security interests. These recent advances include 
- new approaches to multi-agent systems that allow us to envision goal-oriented agents and event responses that span the HSBC domain, as well as modern frameworks and architectures that support models using agents that obey rule sets informed by modern social theory;

- advances in high-performance computing (HPC) resources that can assist computationally intensive cases when large numbers of agents are required to adequately describe regional stability and when aggregation obscures causal factors;

- emergence of architectures that can handle $10^{8}$ agents, disaggregated to the level of individuals behaving according to modern social theories within community scales important to regional stability, while still providing useful predictions in computationally tractable times.

Key to the next generation of social modeling and simulation is the ability to (a) recognize and characterize a surprising event that threatens or impacts a region's stability, (b) model historical events that led to the event with sufficient transparency to allow insight into causal factors, and (c) use this insight to evaluate diplomatic, information, military, and economic (DIME) options for increasing regional stability by influencing emerging events.

In Chapter 5 we illustrate the feasibility of operating such a complex agent-based simulation system that can generate courses of action in computational times much less than 24 hours. The approach to this task included

- estimating the maximum hypothetical complexity of the agents that provides for each dimension of the HSBC, incorporating elements of modern social theory;

- characterizing the maximum complexity of the environment that allows for realtime updating of the environment with unfolding events;

- developing literature examples of analogous systems of comparable complexity;

- comparing employed computational resources to those currently available with similar run times for case studies.

Disaggregated models that also track the motion of individual social atoms are computationally intensive. The complexity of moving resources and people is compounded by increasing granularity in data that allows inclusion of finer behavioral dynamics, thereby increasing the computational intensity. Given that dynamic planning and operational support require large-scale simulations within narrow time windows, reducing execution times is the most critical challenge and can be met only with significant computing resources beyond desktop environments. High-performance computing is, therefore, a necessary enabler for simulations that generate multiple courses of action for a large social region.

Testing the 31-million-agent model of Afghanistan's population demonstrated the ability to computationally control and analytically manipulate a system with the large number of 
agents (i.e., $10^{8}$ ) necessary to model regions at the individual level. Simulations in computationally tractable times can be performed with computational costs that range from less than a dollar for simulations with simple interactions to a few hundred dollars for the largest and most complex simulations. 


\section{Chapter 1: Evaluation of Data Availability and Worldwide Survey of Data Quality}

\section{Research Objective}

The research plan for the ACSES project is provided in Appendix 1 -Research Plan. The objective for Task 1 was to identify and characterize global data sources and the granularity needed to synthesize and model populations at the level required to support the simulation environment. The metrics for this task are

- Demonstrate, or document the feasibility of, direct use of observed or simulated data to seed the behavioral dynamics governing heterogeneous agents representing the entire Human, Social, Behavioral, Cultural (HSBC) domain at each scale of aggregation.

- Demonstrate or document the adequacy of available public domain or classified data sources that relate directly to individual human behavior as well as overall social or economic phenomena, with the objective of guiding the formulation of CAS-based models capable of generating actionable predictive insights and modeling surprising emergent behavior.

- Demonstrate or document the adequacy of disparate, multiscale, and possibly dynamic data sources that can validate the causal patterns and the predictive insights extracted from CAS-based models for social or economic systems.

- Demonstrate the feasibility of utilizing the observed and simulated data in a scalable fashion within a realistic modeling and simulation environment. Specifically, demonstrate the feasibility of enabling the online data-model feedback loop in an environment with more than a 100 million agents, with complex behavior based on realistic social or economic theory, and with disparate data feeds coming in at real-time or near real-time.

In summary, for the seedling effort, Task 1 seeks convincing evidence that the data and related tools exist to construct a synthetic population for the simulation tasks. As a first step, we assessed data quality and then illustrated the concepts by constructing a synthetic population. The criteria used to assess the data quality follow.

\section{Definition of Data-Quality Levels at the National Level}

The criteria for assigning a data quality level are based on the ability to use the assembled data sources to build synthetic populations. Scores of $0,1,2$, and 3 are assigned to variables describing geographical territories. This scale reflects the approximate difficulty in building synthetic populations that support a wide range of potential social theories. 
Areas assigned the value 0 have no data available for building synthetic populations. Examples of such areas include Antarctica and the Australian Indian Territories.

Areas assigned a value of 1 have below-average data quality. In general, these countries are characterized by infrequent or nonexistent census activities and include the central African countries; countries whose data submissions to the United Nations are under suspicion of gross manipulation, such as Cuba and some Former Soviet republics; and a few Central and South American countries. Conflict zones typically fall within this category because of the transient nature of their populations and the difficulty in collecting data in conflict zones. Exceptions include areas where U.S. interest in the political and social stability is high (e.g., North Korea, Iran, Iraq and Myanmar).

Areas assigned a value of 2 have average data quality. These countries routinely submit data to the United Nations, and the data is generally useable with only ordinary qualityassurance checking. Census surveys and data reporting may be less frequent than in North America or the European Union. This category includes most of the developing countries and relatively small participants in world trade flows.

Areas assigned a value of 3 have better-than-average data quality. Census, military structure, economic, social, and infrastructure data are collected annually with known accuracy and precision. This category comprises North America and the European Union, members of the OECD, and major trading partners.

\section{Definition of Independent Variables}

The data required to build a synthetic population can be organized into six broad dimensions - political, military, economic, social, information, and infrastructure-that are important for understanding how populations react and respond to changes in their environment. Within each dimension, the ACSES social theorists surveyed various models and cataloged the independent variables that would satisfy a large fraction of the modeling and surveying needs. These variables are listed in Appendix 2- Data Sources for Independent Variables, where representative sources are also presented to demonstrate source availability.

\section{Assigning Data-Quality Levels at a Dimension Level}

Within a dimension of the independent variables, the following criteria were used to assign the data-quality values.

Political. Almost all countries meet the criteria for data quality level 1, which is identifiable national legislative, executive, and judicial bodies, roles, and representation. Data at this level are of equal or better quality than data in the CIA Fact Book. Data quality level 2 is reached if data exist to interpolate or derive political attitudes and beliefs. Regular polling, census, or anthropological studies that define a time series of loyalties may provide this data. Likewise, information on subpopulations that is derived from data on social cleavages (as determined from social variables) may enable sub- 
national detailing of political ideology, freedom of action, and resources. Data quality level 3 requires that data be available to describe or interpolate the regulation and actions of governmental and nongovernmental organizations as well as their motivation, intent, and objectives. Most close U.S. allies meet the criteria for data quality level 3.

Military. The military dimension of a synthetic population can be separated into two components: (1) the make-up, reliability, and capability of the country's armed forces and (2) attitudes and beliefs about a country's security. Values of 0 or 1 are assigned to areas of active conflict for which little data exists on the make-up, structure, or force level of combatant forces, the acceptance of these forces by the population, or the popular attitudes and beliefs regarding these forces in their roles as peacekeepers, humanitarian support, or a positive influence on general well-being. A data quality level 2 is assigned to countries participating in UN peacekeeping activities or to countries where other data allows a social cleavage analysis of the armed forces. A data quality level 3 is assigned to countries that have entered into mutual security arrangements with the United States and to countries that have been topics of a classified National Intelligence Estimate to address these factors.

Economic. The data quality levels for the economic dimension are highly dependent upon the occupational distribution of the population and the granular distribution of economic activity within the country. Therefore, the assigned quality level for each country was no greater than the LandScan Global data quality level for that country. The methodology for generating the LandScan Global data is described in Appendix 3 - LandScan Methodology. Members of the World Bank and countries contributing data to the International Monetary Fund development and statistics database typically have data that, when combined with other sources, is sufficient to address economic welfare and a leader's ability to allocate resources. Exceptions are nations that have been the subject of classified National Intelligence Estimates that address these factors. In those cases, a data quality level 3 has been assigned.

Social. Data in the social areas can be considered a fusion of data from other domains. However, we have called out this dimension separately because many of the interesting social theories are highly dependent on the variables in this domain. The social dimension depends not only on this fused data and open sources of collected data (Appendix 2), but also is heavily dependent upon attitudes, beliefs, and cultural values that can be developed only by Human Terrain methodologies. As a proxy for these attitudes and belief mapping, we used Gallup World Poll data and the Gallup methodology to make an open assessment of the information available to populate the attitudes and beliefs of a synthetic population. A data quality level of 1 was assigned if the Gallup World Poll was not available within that country and supplemental data sources were not available for human terrain mapping; a level of 2 was assigned if the country had only Gallup World Poll coverage; and a level of 3 was assigned if the polling coverage was supplemented with continuing coverage from additional sources. Appendix 4 - Narrative Description of Key Data Sources describes some of these supplemental sources, the polling methodology, and some examples of the allocation of attitudes and beliefs. 
Infrastructure. The data quality levels for infrastructure were based primarily on engineering sufficiency of the data to allow the performing community to build models of the key infrastructure sectors listed in Appendix 2. For most sectors, the necessary data exist within the U.S. Department of Defense (DoD) mission planning community. This all-source data is currently being supplemented by NGA's open-source infrastructure resources. For example, NGA's Palanterra, a Web-based interface designed to describe, assess, and depict physical features and geographically referenced activities, integrates disparate stores of spatial data to create a spatially and analytically enabled operational picture. Access to the database can be gained at

https://palanterra.nga.mil/PalSec/jsp/JTXRequestAccess.jsps

The data quality level reflects a qualitative assessment of the data available through Palanterra.

Information. The criteria for data quality in the information dimension were based on a qualitative assessment of the ability to model the telecommunications infrastructure sector. For most of the sectors, the necessary data exists within the DoD mission planning community. The open-source baseline data are available in the World Bank's Little Data Book on Information and Communication Technology 2007, and this is supplemented by resources like NGA’s Palanterra.

\section{Maps of Data Quality by Dimension}

Country-by-country data quality levels as assessed using the criteria above are shown in the following maps (Figure 1-1) for each dimension. In Appendix 5, the values for each country's dimensions are presented in tabular form.

Figure 1-1. Quality of data needed to build a synthetic population, evaluated on a country-by-country basis for the following dimensions: (a) political, (b) military, (c) economic, (d) social, (e) infrastructure, and (f) information. 
(a)

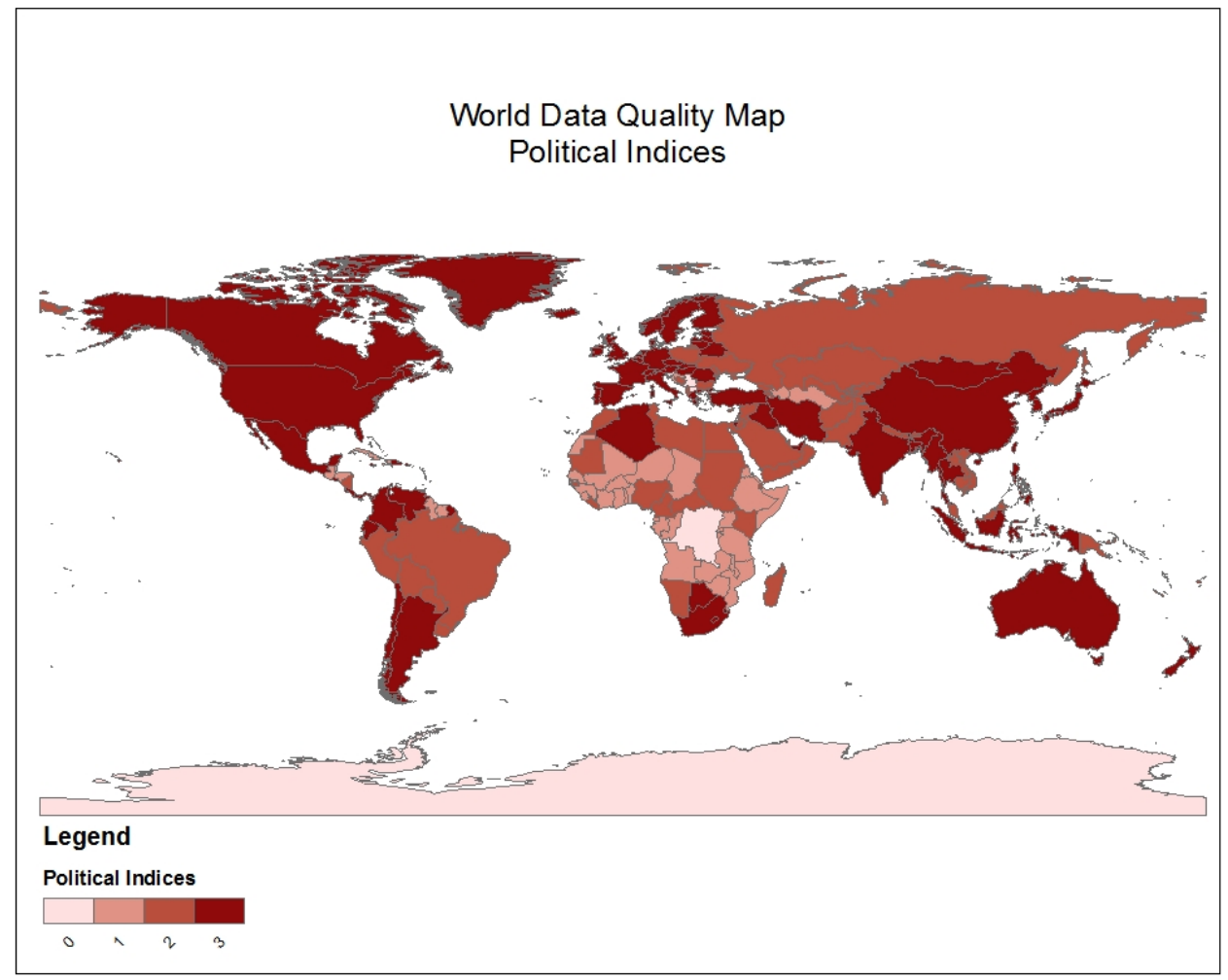

(b)

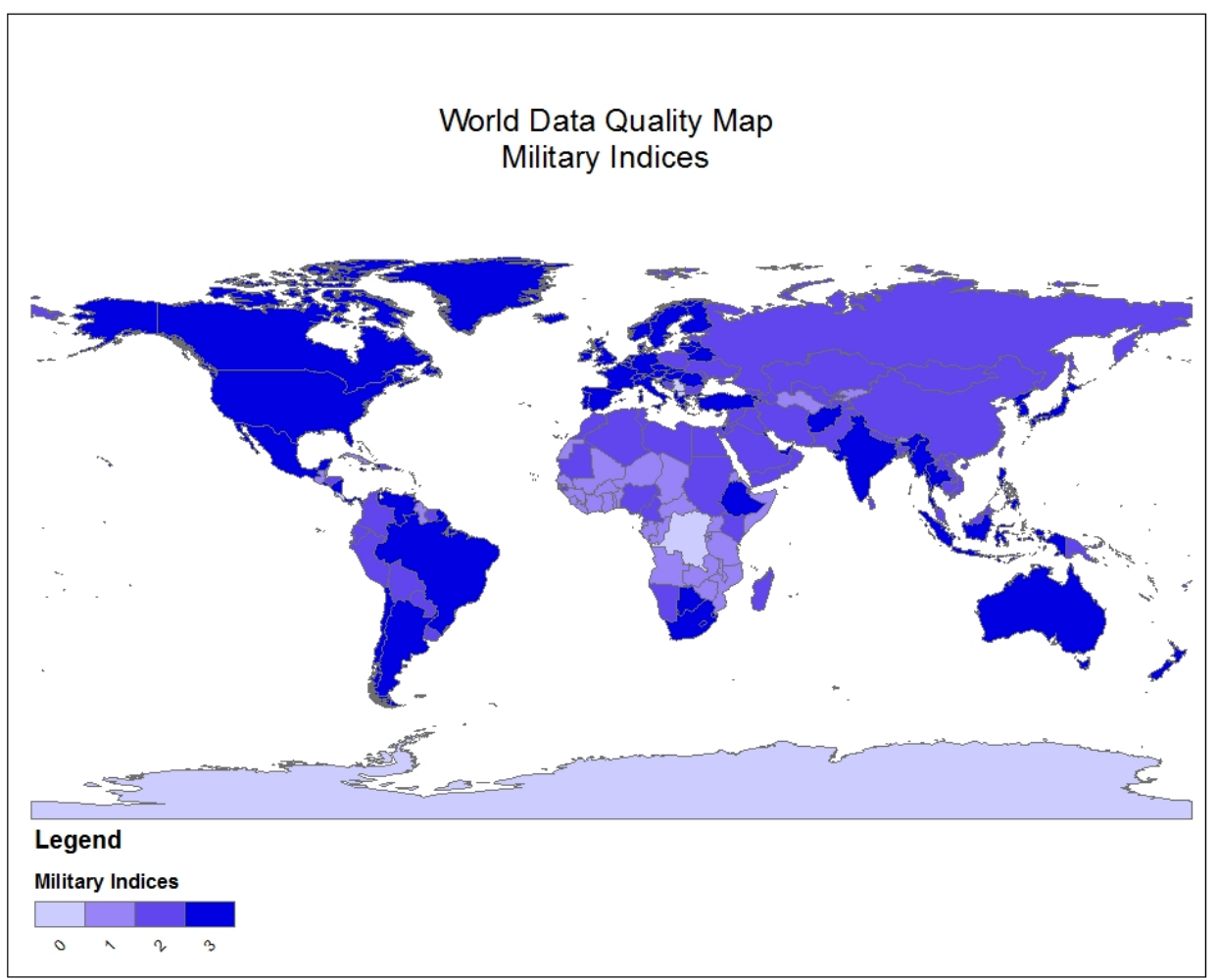


(c)

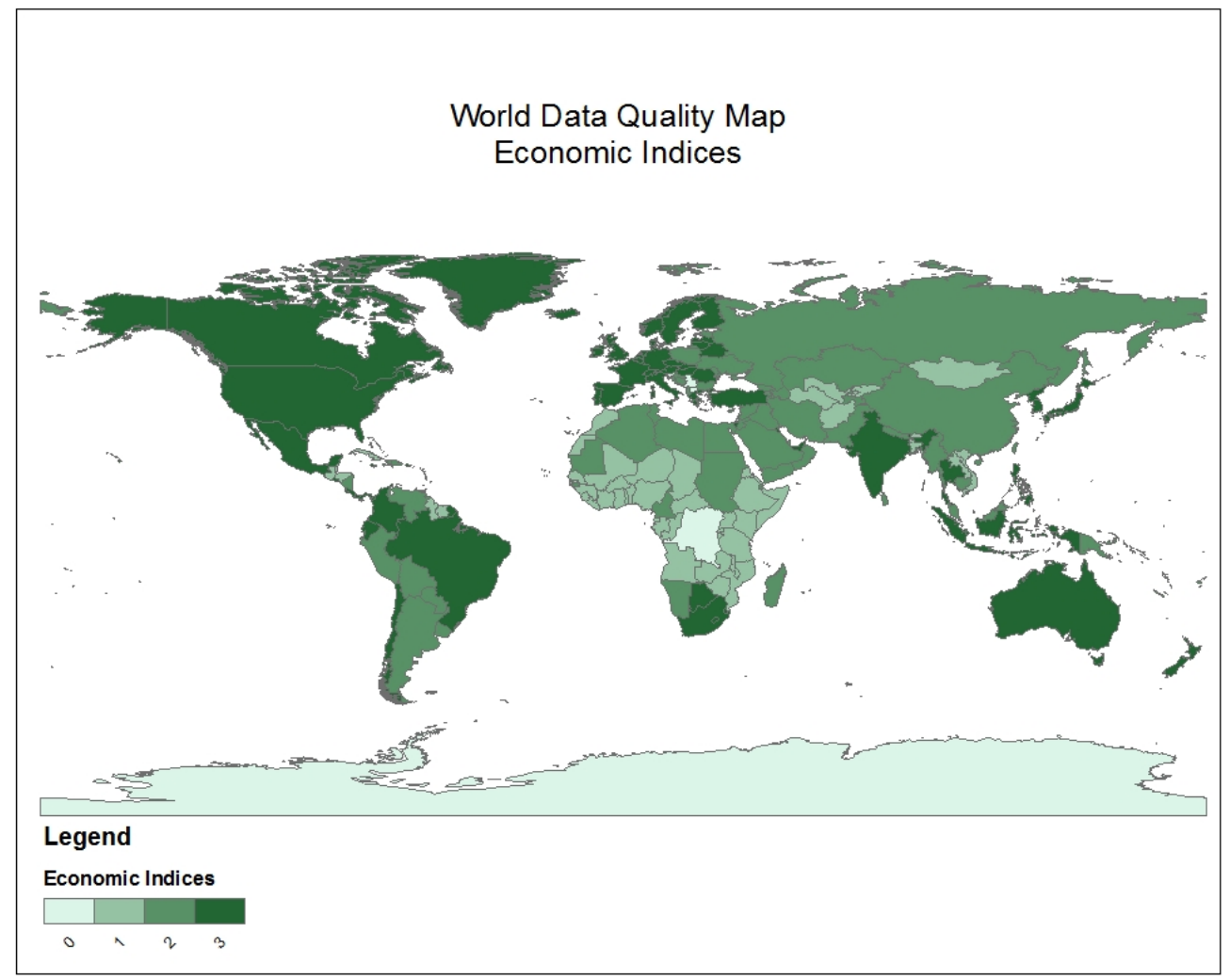

(d)

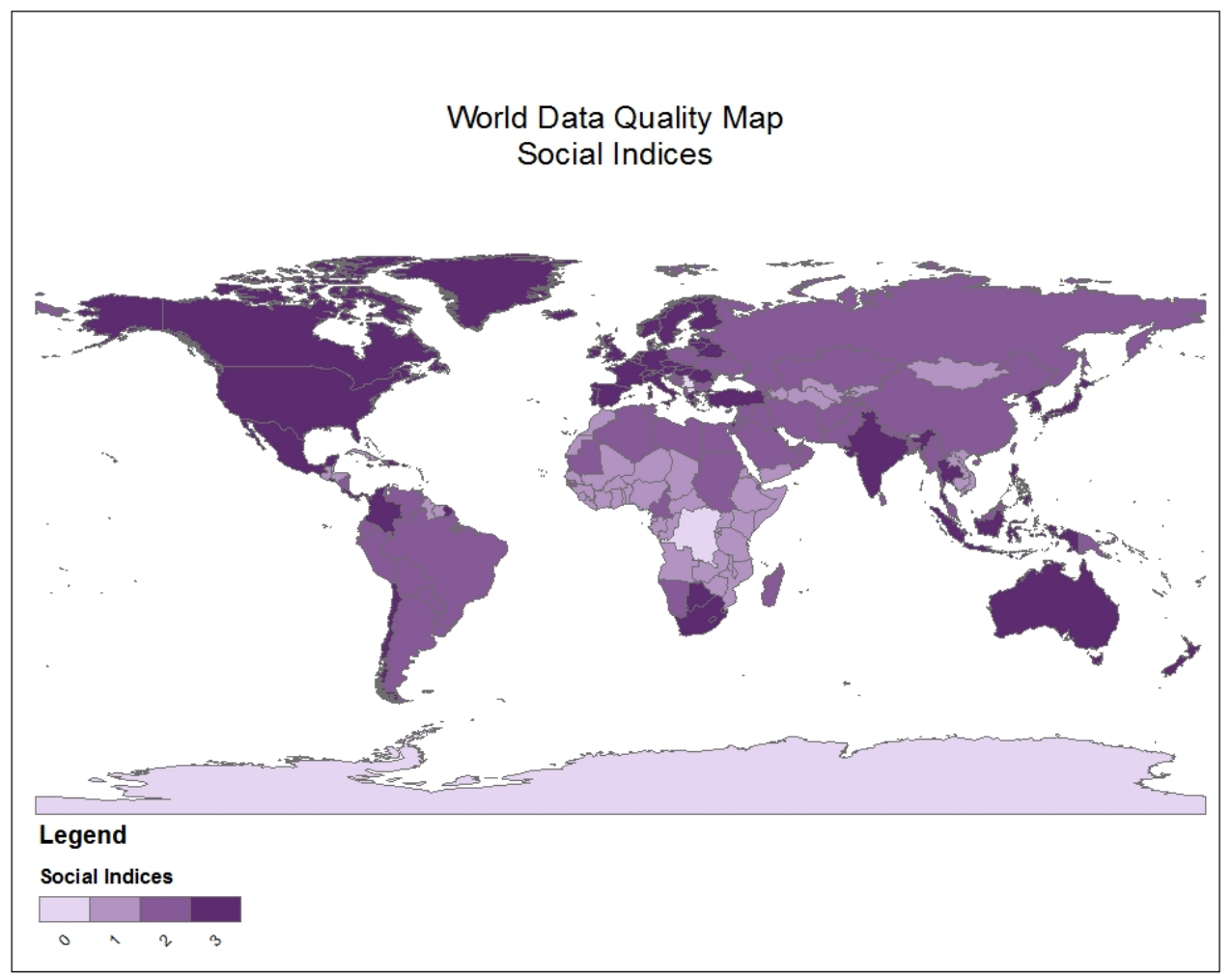


(e)

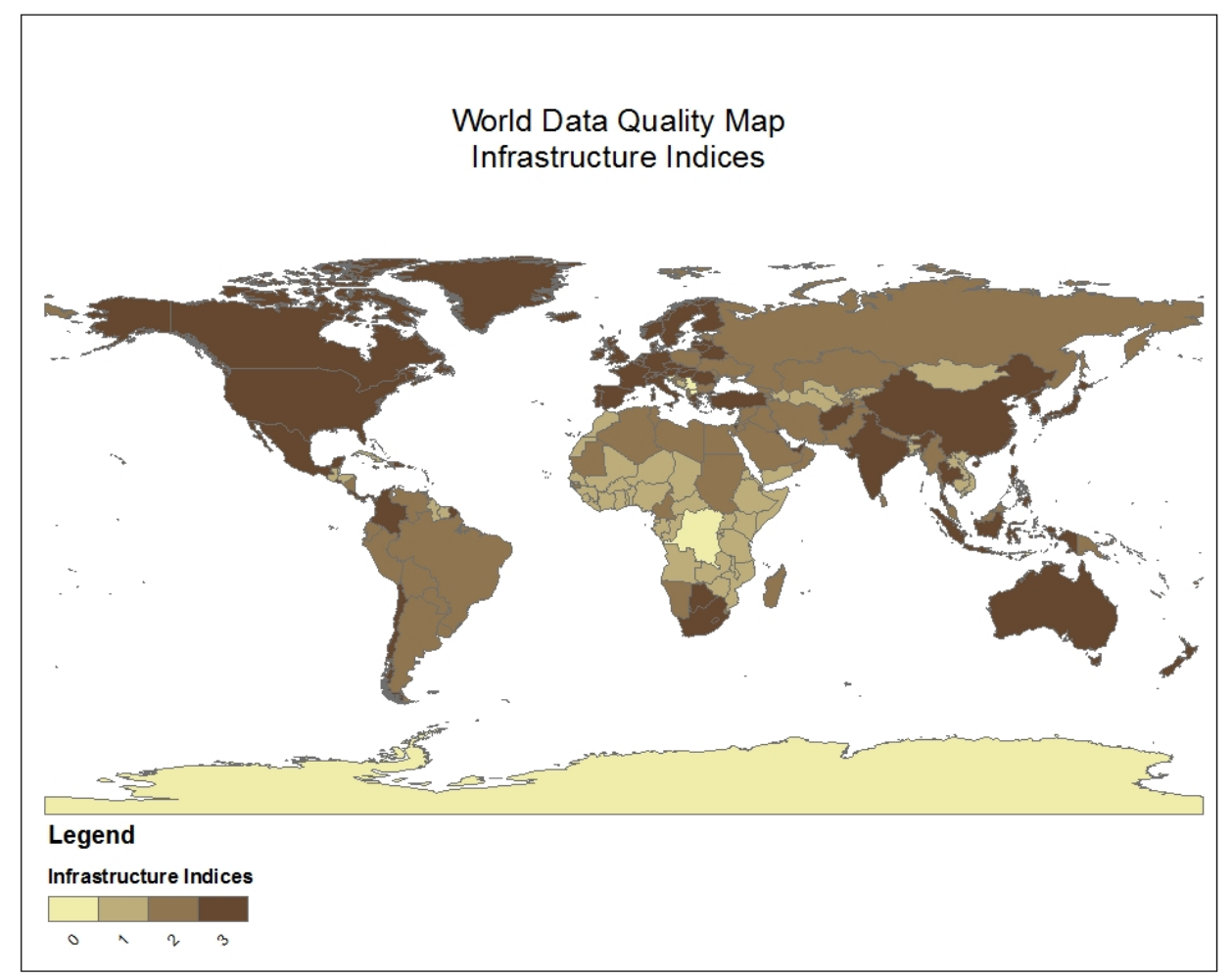

(f)

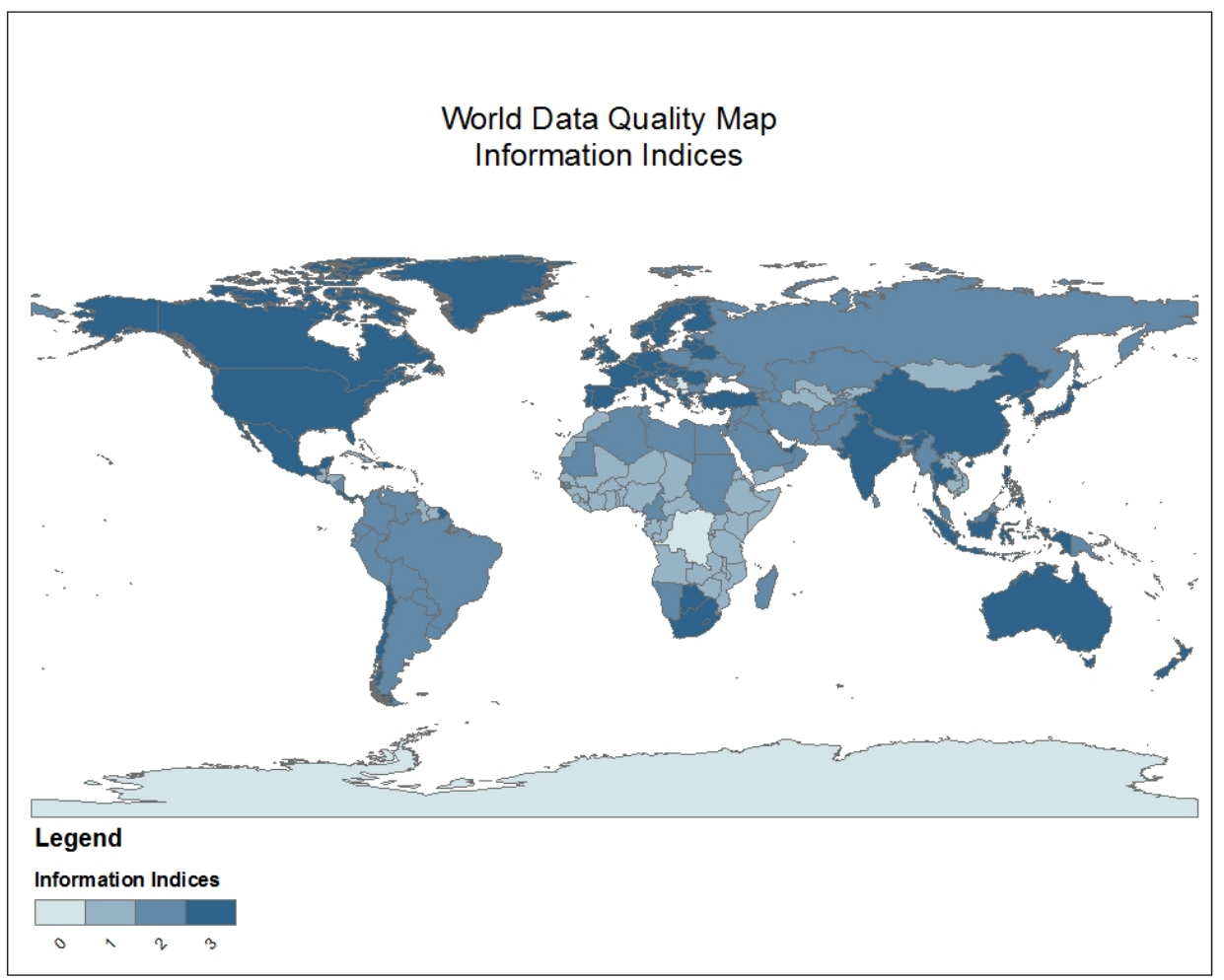




\section{The Afghan Example}

As an example, we constructed a synthetic population representative of Afghanistan's 2006 population, with the ultimate objective of testing the ability of various leadership theories to answer strategic questions. One of these questions asks how to reduce the overall level of terror incidents. In this case, the leadership theories require input on loyalty, coercion, ideology, economic welfare, and security from violence for each agent of the population. To geospatially assign attributes for the agent's support for the leadership, the leadership's resources, the agent's ideology, and the agent's degree of satisfaction with the economic and security situations, databases and data sources were employed according to Figure 1-2.

Figure 1-2. The flow of data source employment in construction of the Afghan synthetic population

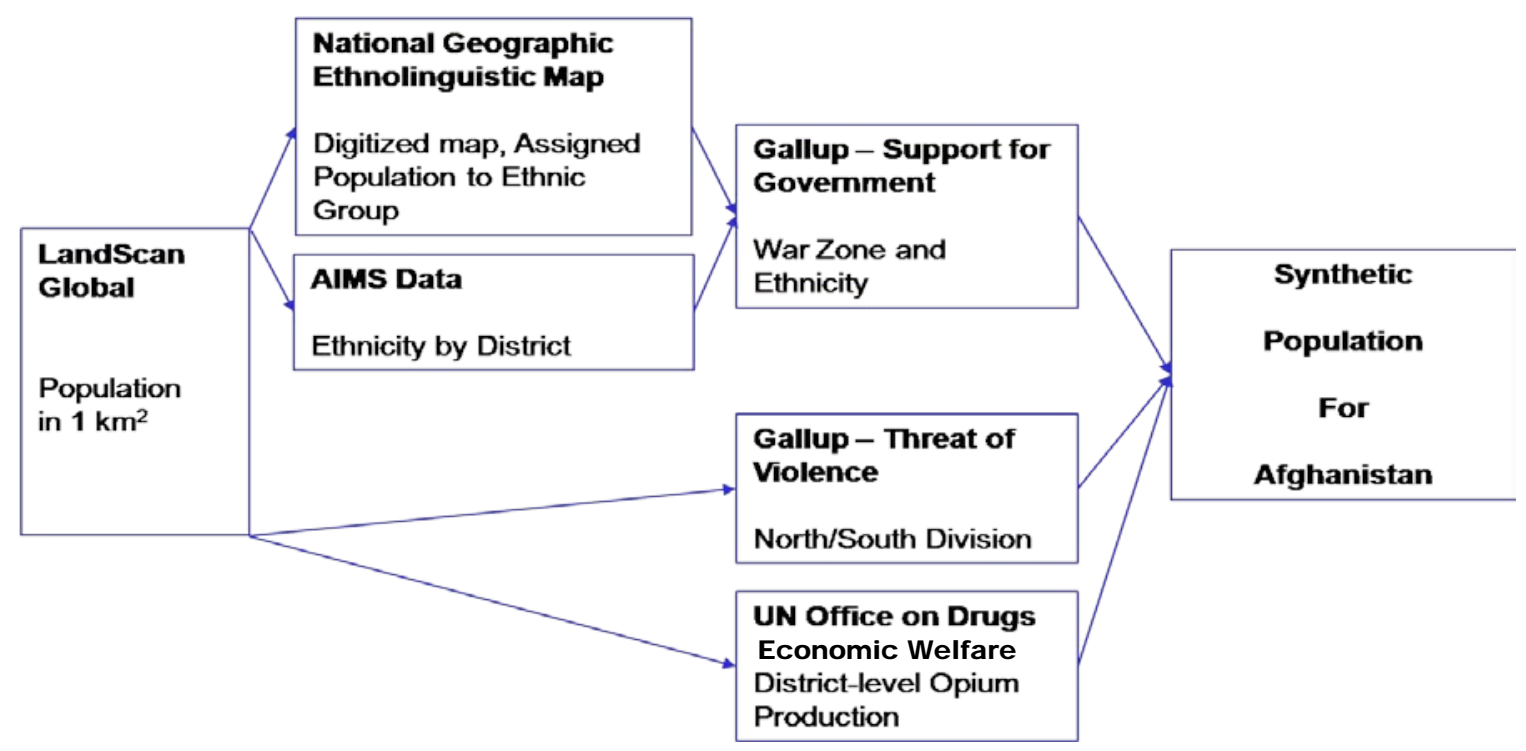


The first step in building the synthetic population was geospatially locating the 31 million agents representing the Afghan population within grids approximately one kilometer on each side. The LandScan Global techniques (Appendix 3 - LandScan Methodology) for disaggregating a population produced the geographical population distribution illustrated in Figure 1-3.

Figure 1-3. Geospatial distribution of agents representing the Afghan population is represented on one square kilometer square cells.

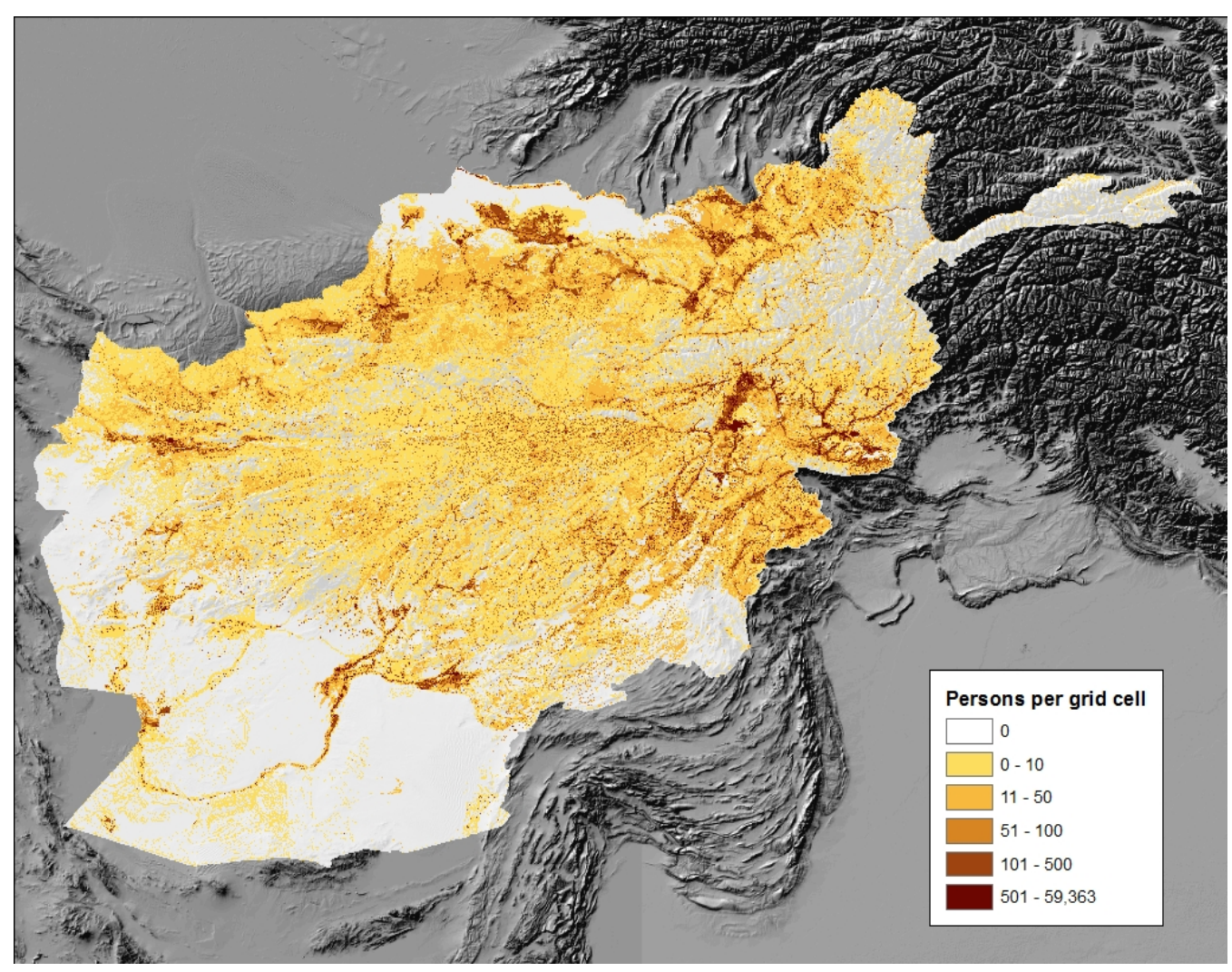

Assigning attributes to this disaggregated population was achieved by fusing appropriate indicator databases. A diverse body of literature describes the major Afghan political and social cleavages that can be used to deduce ideology and perceptions of security against violence. We began by consulting district profiles (example in Appendix 4 - Narrative Description of Key Data Sources) collected through human-terrain-type efforts by the Afghan Information Management Service (AIMS) from which we can determine the ethnicity of each element in 206 of the 329 Afghan districts. For the remaining 123 districts, the ethnic breakdown was estimated by interpolating data on ethno-linguistic maps, such as in Figure 1-4, that delineate the distribution of ethnic groups. Ethnic data from adjacent districts was used for the interpolation when it was available. Otherwise, data from nearby areas sufficed. Although many granular maps exist, the National 
Geographic maps were used to demonstrate the value of common maps of limited granularity.

Figure 1-4. Ethno-linguistic mappings used for ethnic interpolation of ethnicities in missing districts. Source: National Geographic.

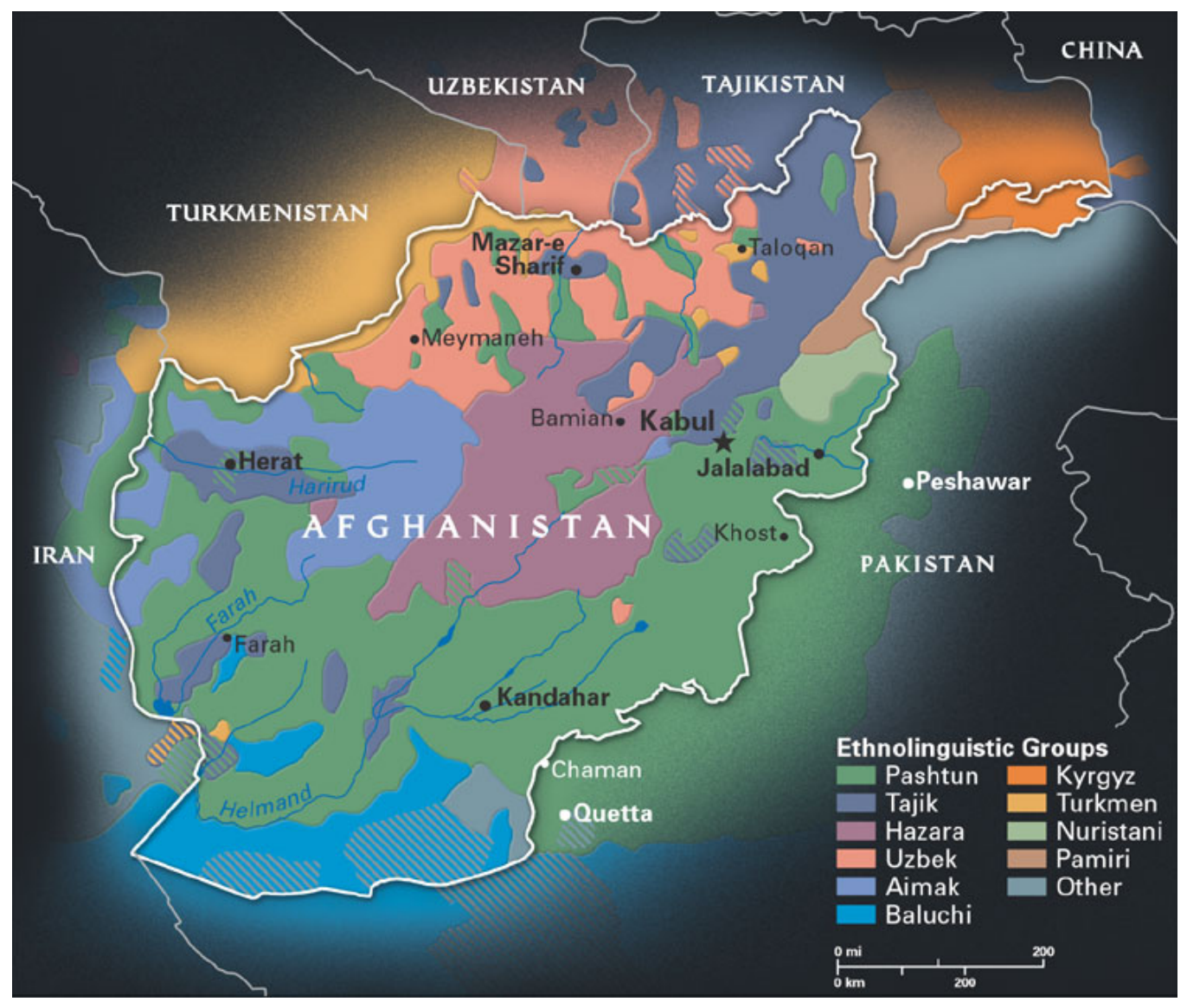

Once the ethnicity for each of the 31 million agents was assigned, then the ideology and attitudes toward the economic and security situation were assigned to each social atom. Although very specific polling data can be gathered in areas of high U.S. national interest, we used existing Gallup World Poll data for this study to illustrate the methodology. The methodology, poll summary, and potential Gallup coverage are described in Appendix 4 - Narrative Description of Key Data Sources. 
The attitudes within the Gallup study group seem to have significant cleavages along ethnic (Pashtun, Tajik, and Other), location (north, south), and combat proximity (war zone vs. nonwar zone) lines. A mapping for war zone location is reproduced in Figure 15.

Figure 1-5. Perceived war zones. Perceptions of violence are dependent upon the agents' locations relative to the war zone.

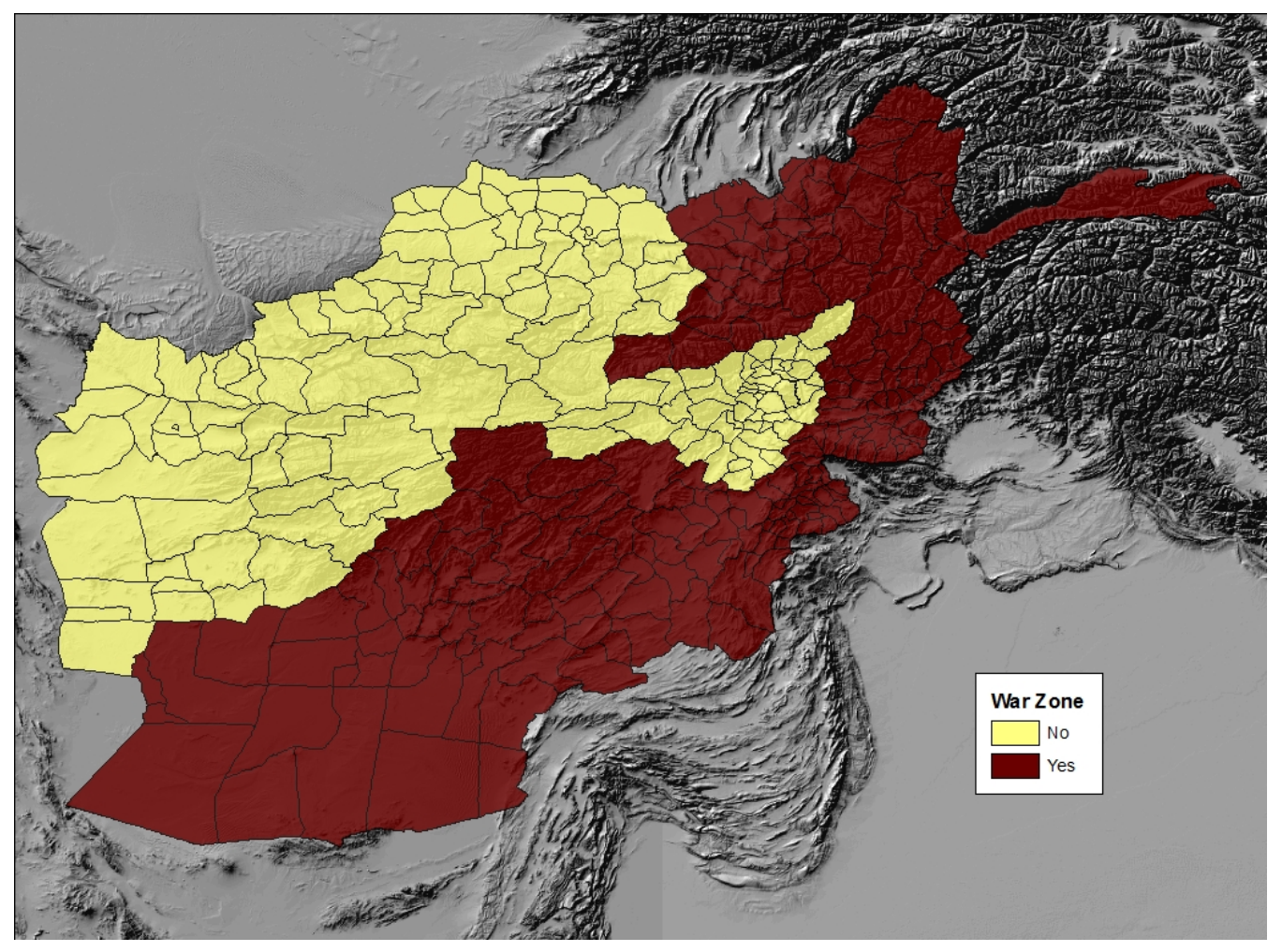


The indicator database for how economic welfare is perceived by the individual and is tied to behaviors in support of leaders in the Afghan case is highly dependent upon the social atom's proximity to opium production (Figure 1-6). The Afghan example is simpler than most countries where corruption indices might create usable indicator databases and where economic activity under leadership control may be more complex.

Figure 1-6. Areas of opium cultivation based on 2004 data from the UN Office on Drugs and Crime. The district leaders' ability to control economic resources correlates with the presence of opium cultivation.

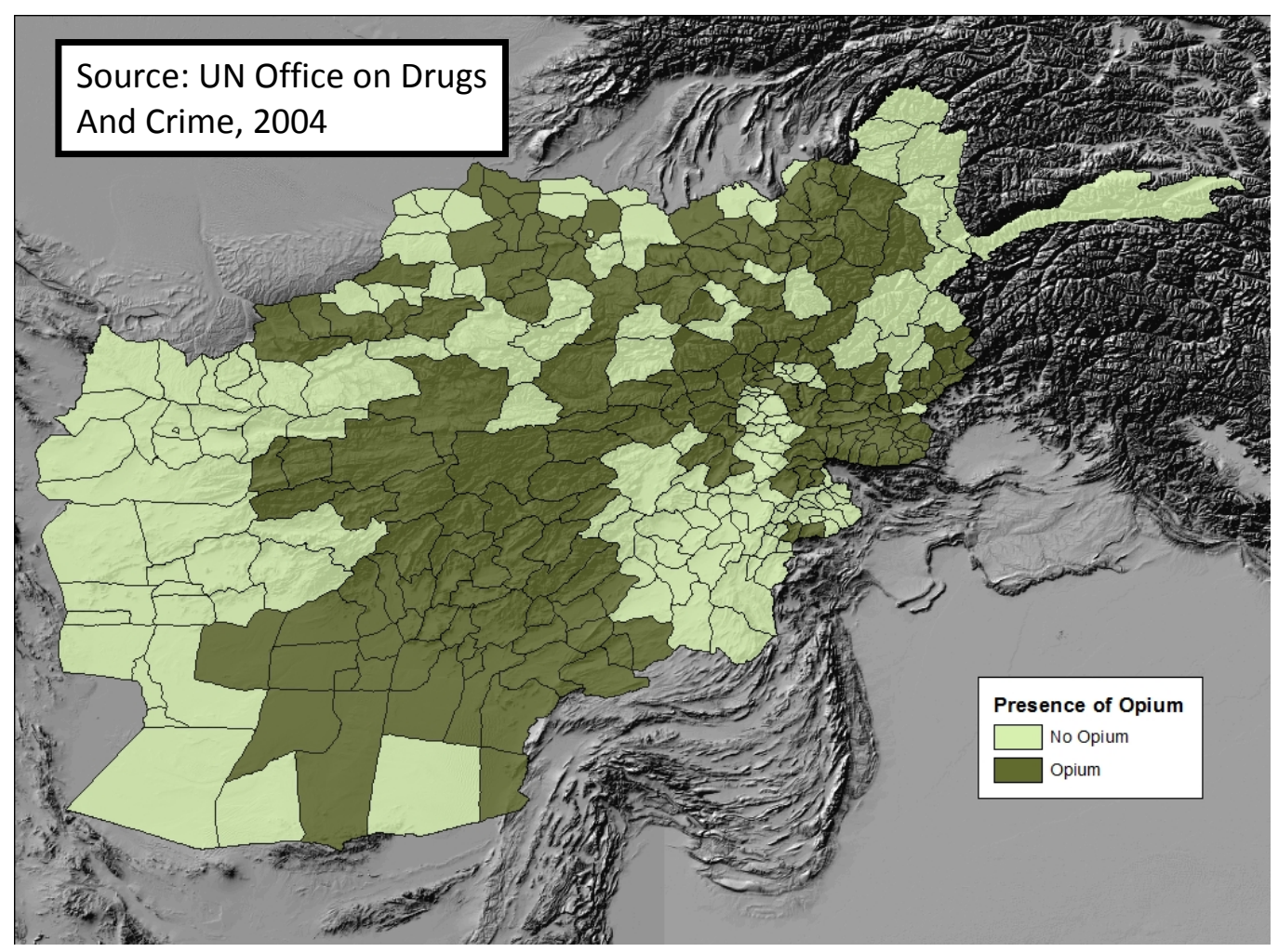

The elements above, taken together, were the building blocks of our synthetic population. Tasks 2 through 5 used this population for simulations that could then be compared to real-world activities.

\section{Lessons Learned and Insights}

In the course of this study, a large number of sources were assembled to acquire information in support of the flexible simulation model. From this study, we have learned that recent government efforts coordinated through the National Geospatial-Intelligence Agency have improved the granularity and coverage of the data required by many social theory simulations. Because motivations and intent of either individuals or small groups are difficult to collect in real-time, databases of populations' spatial distributions and 
behaviors require demographic experts to apply data fusion, knowledge discovery, and demographic modeling techniques to build synthetic populations.

Fortunately, these techniques exist, and active federal research programs are further developing the techniques. Validation studies on denied areas of the world are continuing to evolve, but a variety of modeling techniques is providing new access to social behaviors in these portions of the world.

In the effort to demonstrate, or document the feasibility of, direct use of observed or simulated data to seed the behavioral dynamics governing heterogeneous agents representing the entire HSBC domain at each scale of aggregation, we have learned it is feasible. Although assigning nonobservable beliefs and attitudes to individual social atoms remains beyond the state-of-the-art, modern demographic techniques such as those embodied within NGA efforts can build synthetic populations with a low number of clones.

In this task, we also attempted to demonstrate or document the adequacy of available public domain or classified data sources that relate directly to individual human behavior as well as overall social or economic phenomena, with the objective of guiding the formulation of CAS-based models capable of generating actionable predictive insights as well as modeling surprising emergent behavior. We learned that a successful effort that includes both political and military HSBC domains requires classified data sources, but synthetic populations can still be constructed.

In addition, this study attempted to demonstrate or document the adequacy of disparate, multiscale, and dynamic data sources that can validate the causal patterns and the predictive insights extracted from CAS-based models for social or economic systems. The classified data sources concerning those areas suffering the greatest regional instability are essential to the construction of synthetic populations. Unclassified sources are adequate for demonstrating certain principles and methodologies and future technical issues. However, the new simulation systems will create demands for survey and data collection in a fashion similar to the Army's Human Terrain collection efforts and the Strategic Command's Human Network Attack capabilities.

As part of Task 1 we sought to ascertain the range of variables for which data can be effectively gathered. A key insight from this initiative is that it is possible to identify and assemble data sources pertaining to many higher-level social concepts such as freedom, corruption, poverty, and empowerment. In most instances these data are calculated and disseminated only at the national level, but data fusion and disaggregation techniques such as those used in this task can be applied to these variables as well. 


\section{Chapter 2: Agent-Based Modeling}

\section{Introduction}

The goal of this project is to develop a simulation capability to monitor, assess, forecast, and respond to adverse human behavioral developments around the world in near realtime and to use the simulations to compare the performance of agent-based models grounded in modern social theory. We review the general agent-based approach in a simulation of Afghanistan and then segue into a review of the three incremental approaches to modeling agents. The chapter begins with a description of the NetLogo simulation, including a description of the country, agents, their rules (which are derived from the utility function), outcomes, and learning.

\section{Social Theories}

This section describes the social theories implemented in the simulation along with the rationale for their use. The rationale involves the domain of the theory (i.e., the social phenomenon it concerns) and scientific justification for the theory.

The social theories implemented concern only the citizen agents because a primary interest, if not the primary interest, of the ultimate users of such a simulation model would be the allegiance of the population. That is, the users, whether social scientists investigating social theories or commanders in the field examining real-world options, would want to know if ordinary citizens actively supported the insurgents, actively supported the government, or neither.

Each theory pertains to one of three domains:

1. The effect of leaders on the behavior of citizen agents (leadership theories)

2. The effect of the behaviors of similar others (neighbors and community) on the behavior of citizen agents (social mobilization theories)

3. The factors influencing how a citizen agent's situation and behaviors may lead to change in the agent's support for the leadership and in the agent's ideology (psychological change or learning theories)

Table 2-1 summarizes the theories implemented in the simulation, as well as their domains, outputs, locations in the simulation, and parameter settings that instantiate the theories. There are a total of nine theories: three leadership theories, two social mobilization theories, and four theories of psychological change, each of the last four implementable for both change in support for a leader and change in ideology. This means that $96(3 \times 2 \times 4 \times 4)$ combinations of theories are possible.

All of the theories are implemented via a model of the agent. The leadership and social mobilization theories are instantiated with parameter settings in the agent model, and the change theories determine inputs to the agent model. Any model of how an individual agent makes behavioral choices can be realized as maximization of a utility function. The utility function incorporates social theories that model human decision processes. 
We chose to model at the level of the agent for several reasons. First, it is possible. Any social process, any social system dynamics, must occur through the behavior of individuals. Models of agents, therefore, together with rules concerning interactions of those individuals, should be able to reproduce system-level patterns. Second, it gives flexibility. Modeling at the individual level allows for exploration of the effects of particular events and individuals. Third, it facilitates development of the simulation model. For example, the simulation model currently allows the user to control the orders and resources of the leadership by region.

In the future, it would be desirable to allow leadership to be endogenous to the simulation. Citizen agents from the population would be allowed to become leaders, at which point they would have a disproportionately large effect on events. Processes such as this are realistic and potentially consequential, and they would be straightforward to model through an agent-based simulation.

Table 2-1. Theories implemented in the simulation model

\begin{tabular}{|c|c|c|c|c|}
\hline Theory & Domain & $\begin{array}{l}\text { Output } \\
\text { Level }\end{array}$ & Location in & Parameter Settings \\
\hline legitimacy & leadership & $\begin{array}{l}\text { individual } \\
\text { behavior }\end{array}$ & $\begin{array}{l}\text { utility } \\
\text { function }\end{array}$ & high loyalty \\
\hline coercion & leadership & $\begin{array}{l}\text { individual } \\
\text { behavior }\end{array}$ & $\begin{array}{l}\text { utility } \\
\text { function }\end{array}$ & high coercion \\
\hline representative & leadership & $\begin{array}{l}\text { individual } \\
\text { behavior }\end{array}$ & $\begin{array}{l}\text { utility } \\
\text { function }\end{array}$ & $\begin{array}{l}\text { high economics, violence, } \\
\text { ideology }\end{array}$ \\
\hline social influence & $\begin{array}{l}\text { social } \\
\text { mobilization }\end{array}$ & $\begin{array}{l}\text { individual } \\
\text { behavior }\end{array}$ & $\begin{array}{l}\text { utility } \\
\text { function }\end{array}$ & high influence \\
\hline $\begin{array}{l}\text { resistance to } \\
\text { repression }\end{array}$ & $\begin{array}{l}\text { social } \\
\text { mobilization } \\
\text { psvchological }\end{array}$ & $\begin{array}{l}\text { individual } \\
\text { behavior }\end{array}$ & $\begin{array}{l}\text { utility } \\
\text { function } \\
\text { component of }\end{array}$ & high repression \\
\hline socialization & $\begin{array}{l}\text { change - to } \\
\text { ideology \&/or } \\
\text { support for } \\
\text { leader }\end{array}$ & $\begin{array}{l}\text { input to } \\
\text { utility } \\
\text { function }\end{array}$ & $\begin{array}{l}\text { utility } \\
\text { function- } \\
\text { ideology } \\
\text { \&/or loyalty }\end{array}$ & soc $>0$ \\
\hline homophily & $\begin{array}{l}\text { psychological } \\
\text { change - to } \\
\text { ideology \&/or } \\
\text { support for } \\
\text { leader }\end{array}$ & $\begin{array}{l}\text { input to } \\
\text { utility } \\
\text { function }\end{array}$ & $\begin{array}{l}\text { component of } \\
\text { utility } \\
\text { function- } \\
\text { ideology } \\
\text { \&/or loyalty }\end{array}$ & $\begin{array}{l}\text { delta (rate) }>0 \text {, } \\
\text { hom }>0\end{array}$ \\
\hline results-based & $\begin{array}{l}\text { psychological } \\
\text { change - to } \\
\text { ideology \&/or } \\
\text { support for } \\
\text { leader }\end{array}$ & $\begin{array}{l}\text { input to } \\
\text { utility } \\
\text { function }\end{array}$ & $\begin{array}{l}\text { component of } \\
\text { utility } \\
\text { function- } \\
\text { ideology } \\
\text { \&/or loyalty }\end{array}$ & delta (rate) $>0, \mathrm{RB}>0$ \\
\hline cognitive & $\begin{array}{l}\text { psychological } \\
\text { change - to } \\
\text { ideology \&/or } \\
\text { support for } \\
\text { leader }\end{array}$ & $\begin{array}{l}\text { input to } \\
\text { utility } \\
\text { function }\end{array}$ & $\begin{array}{l}\text { component of } \\
\text { utility } \\
\text { function- } \\
\text { ideology } \\
\text { \&/or loyalty }\end{array}$ & delta (rate) $>0, \mathrm{CD}>0$ \\
\hline
\end{tabular}




\section{The Leadership Theories}

The leadership theories concern the effect that the leadership of a group has on the allegiance - pro-insurgent, pro-government or neutral—of the members of the group. We implemented these theories in the simulation model because considerable social theory says that leadership has important effects on people's behavior and because empirical reports concerning conflict in Afghanistan and Iraq, as well as in many areas of social conflict throughout the world, also stress the important effects of leadership. In particular, the effects of leaders on the population almost certainly would be important elements of many questions a user would want to ask of the simulation model. It is hard to imagine any scenario of interest in Afghanistan, for example, that would not involve the effects of leaders and would not consider them consequential. The three leadership theories implemented are all common views in social science of the effects leaders have. The theories may be considered alternatives and compared, or they may be treated as complementary and combined.

The legitimacy theory posits that a citizen agent wants to follow the orders of the leadership simply because it is the legitimate leadership. This idea is common in the social science literature. It corresponds to the concept of authority either granted willingly by an agent, or delegated or enforced (Coleman 1990). It fits the idea of legitimacy granted to group members who have certain characteristics or demonstrate certain abilities (Berger et al. 1998). It also can encompass Weber’s (1968 [1922]) concept of charisma, according to which some individuals have qualities that induce people to follow them.

The coercion theory posits that a citizen agent wants to follow the orders of the leaders because he or she believes they are likely to punish those who do not obey or to reward those who do. This theory can be traced back at least to Machiavelli, who advised in The Prince (Machiavelli 1985 [1513]) that rulers could be most effective by making their subjects fear them, and also has contemporary advocates (e.g., Kiser and Linton 2002; Levi 1988). It fits the behaviorist perspective in psychology (e.g., Sidman 2000) and deterrence theory in criminology (Becker 1968; Stigler 1970).

The representative theory posits that a citizen agent follows the leadership only if the leadership advocates what the citizen agent otherwise wants. Thus, the leadership's orders have only a weak effect on the behaviors of the population. This idea goes back at least to Marx (1990 [1867]), who saw the government as merely implementing the desires of the ruling class, but it also has been argued for recently, for example, in the context of Latin American rebellions and popular nationalism (Whitmeyer 2002; Wickham-Crowley 1991).

All three of these theories are likely to be useful to an operator using the simulation model. The validity and effects of the legitimacy theory are relevant, for example, to the question of how important it is to work through traditional and legitimate leaders of the 
population in combating insurgency. The validity and effects of the coercion theory are relevant to the question of the likely effects of terrorist activities by the insurgency and how to deal with local strongmen. The representative theory is a strong alternative if the other two theories are not acceptable.

\section{The Social Mobilization Theories}

The simulation model implements two social mobilization theories that concern the effects of social influence on participation in collective action (Table 2-1). These two theories are clearly relevant to the allegiance - pro-government, pro-insurgency, or neutral behavior —of the population in a country with significant internal conflict. We implemented these theories in the simulation model because considerable empirical evidence shows that people's participation in collective action is affected by the behavior of those around them and those close to them. This is true around the world, for example in the United States (Roscigno and Danaher 2004), in the former East Germany (Opp and Gern 1993), and in recent China (Calhoun 1994). Many theoretical models of collective action exist, and most if not all of them incorporate the effects of social influence (Oliver 1993; Ostrom 2007).

Inclusion of the social mobilization theories gives the simulation model more social realism directly relevant to the outcome the simulation generates, allegiance. People are not independent, autonomous actors, but rather affect each other. This may mitigate or exacerbate the effects of their situation and, as research has shown, is likely to be responsible for nonlinear and emergent effects in social phenomena generally (Coleman 1990) and in collective action in particular (Ostrom 2007). The social influence theory posits that a person is more likely to join collective action the more his or her immediate friends do (McPhail 1991, 1994). This concept originated in the study of riots. The repression theory is complementary to the social influence theory. It posits two effects. First, people are less likely to join a collective action the greater the local presence of forces to repress it. Second, in the presence of some repression, people are more likely to join collective action the larger the proportion of people in the observable population who are engaged in this action.

These effects are shown, for example, by studies of the overthrow of the East German regime (Opp and Roehl 1990; Opp and Gern 1993). They are also present in the critical mass idea that often collective action can accelerate once a critical mass of people has joined (Marwell and Oliver 1993). This theory is likely to be used in tandem with one of the leadership theories as well as with the social influence theory. Again, the weights instantiating these theories will have to be reduced proportionally as $w_{R}$ is raised. The effect of these adjustments in the model is that (1) the citizen agent will tend to have a lower utility for behaviors that are opposed by the forces controlling the agent's locality, but (2) this effect will be ameliorated to the extent that the agent observes other citizen agents engaging in the behaviors.

Both of these theories are likely to be useful to an operator using the simulation model, especially in conjunction with one of the leadership theories. Neither social influence nor 
repression is likely to initiate pro-government or pro-insurgent activity. For that, some other cause is needed, such as an order from the leadership. The repression theory adds, however, the ability of local force to suppress unwanted activity, and both theories bring in people's influence on each other, which introduces the possibility that activities can spread across a population. These features will increase an operator's ability to use the model to assess the efficacy of measures and countermeasures.

\section{The Relationship between Social Theories}

The leadership theories and social mobilization theories are at the level of the human actor; they concern the choice of behavior. The change or "learning" theories are theories of psychological change and are at a more micro level; they are internal to an individual. There are four change theories for ideology and the same four for support for the leadership. In each area, the four theories are alternatives. They can be combined, but necessarily the more important one is, the less important the others will be. The two areas, ideology and support for the leadership, are completely independent. Thus, one theory could be implemented in one area and a different theory in the other area. The change theories are also not alternatives to the leadership or social mobilization theories because they are at a lower level. The variables the change theories affect feed into the utility function that implements the actor theories.

The social mobilization theories are functionally alternatives to each other and to the leadership theories, because they all concern what values or motives affect behavior. Specifically, they are all implemented as weights for values in the utility function, so if the weight implementing the social influence theory is greater, the sum of the weights applied to a leadership theory and the repression theory must be lower.

Conceptually, however, the social influence theory, the repression theory, and any given leadership theory are not alternatives. That is, it is not the case that the more one theory fits an empirical situation, the less the others do, which is the case between the three leadership theories. Rather, the more important that one theory (say, social influence) is in an empirical situation, the less important the other theories (the leadership theory and the repression theory) will be. The other theories may fit and help explain the situation but may just be less important.

Finally, the effects of the change theories and the actor theories are not independent, of course, in the sense that they are not additive. For example, the change theories concerning support for the leader matter if the legitimacy theory is implemented, but they do not matter much if the representative theory is implemented.

\section{Utility Function}

One of the key challenges was finding a way to allow multiple social theories to participate in the same simulation so that could be compared in situ, and without writing a great deal of new code. The linchpin that ties together the various social models we incorporated is the utility function. 
Many forms of utility functions have been devised. We chose the Cobb-Douglas utility function, which specifies utility as the product of values (i.e., preferences, motivations) raised to a fractional power such that the exponents sum to 1 [Eqn (1)]. An agent chooses to perform one behavior, from a set of considered behaviors that has the highest expected utility. This utility function has been used for instantiating a variety of social theories (Coleman 1990) and has several desirable features. It is simple. It is modular, thus easily expandable to include additional values or motivations if they are important for a theory.

Moreover, even if the number of values in the utility function rises to a large number, any value can be turned off by setting its parameter to 0 , so that if desired, users can operate with what is effectively a much smaller utility function. Finally, the model takes into account the important experimental finding that losses tend to affect people more than gains (Tversky and Kahneman 1981). Mathematically, this means that the second partial derivative of the utility, $U$, with respect to any given value is negative. Eqn (1) gives the utility function that is currently implemented for citizen agents in the simulation model:

$$
U=(1-L)^{w_{L}}(1-C)^{w_{C}}(1-I)^{w_{I}}(1-E)^{w_{E}}(1-V)^{w_{V}}(1-F)^{w_{F}}(1-R)^{w_{R}} .
$$

$L$ (loyalty to leader), $C$ (coercion), $I$ (ideology), $E$ (economic welfare), $V$ (security against violence), $F$ (influence of close associates), and $R$ (repression and social influence for defying repression) are the deviations from an ideal state (or "motives") corresponding to the seven different values currently implemented. The $w$ 's are the weights of different values in the utility function. The weights are required to be nonnegative, to be less than or equal to 1 , and to sum to $1\left(w_{L}+w_{C}+w_{I}+w_{E}+w_{V}+w_{F}+w_{R}=1\right)$. Their particular values are parameters set by the user and instantiate different social theories and combinations of theories, as detailed in the descriptions of the theories in the following sections.

The user implements the legitimacy theory by setting the parameter $w_{L}$ to a relatively high value, i.e., giving high importance to the loyalty component of the utility function. This means that the citizen agent will tend to have a lower utility for behavior the more the behavior runs contrary to the leadership's orders.

The user implements the coercion theory by setting the parameter $w_{C}$ to a relatively high value, i.e., giving high importance to the coercion component of the utility function. This means that the citizen agent will tend to have a lower utility for behavior the more it runs contrary to the leadership's orders to the extent that the leadership has a high level of resources.

The user implements the representative theory by setting the parameters $w_{I}, w_{E}$, and $w_{V}$ to relatively high values, i.e., giving high importance to the ideology, economic welfare, and security against violence components of the utility function. This means that the orders of the leadership, even if the leadership has ample resources, are likely to have little effect on the behavior of the citizen agent compared with the effects of the citizen agent's situation and own predilections. 
The user implements the social influence theory by setting the parameter $w_{F}$ to a relatively high value, i.e., giving high importance to the influence-of-close-associates component of the utility function. A second parameter sets the radius of social influence affecting a single citizen agent. This theory is likely to be used in tandem with one of the leadership theories, but the weights instantiating the leadership theory will have to be reduced proportionally as $w_{F}$ is raised. The effect of these adjustments in the model is that the citizen agent will tend to have a lower utility for behaviors the more they are contrary to the behaviors of those close to the agent.

The user implements the repression theory by setting the parameter $w_{R}$ to a relatively high value, i.e., giving high importance to the repression component of the utility function. A second parameter sets the radius of the area a citizen agent observes to see how prevalent a pro-government or pro-insurgent behavior is.

We will show the feasibility of developing an evaluation, characterization, and validation framework that, when fully developed, will be able to provide guidelines to a decision maker regarding the best choice of theory or combination of theories. The recommendation would be based on an initial analysis of prevailing conditions in a new country, answers provided by end users and subject matter experts to targeted questions, as well as end user requirements.

\section{Geographical Presentation}

The country is divided into six regions, and each region is divided into patches (Table 22). The regions are simple and somewhat arbitrary divisions of the country. The purpose of delineating six regions was to enable multiple leaders for the Pashtun tribe such that each Pashtun leader had a geographic-based influence on Pashtun agents. Therefore, the regions apply only to the Pashtun tribe. Other tribes have only one leader each, and those leaders have influence on their agents across the entire country.

Table 2-2. The NetLogo regions

\begin{tabular}{|c|c|c|}
\hline Region & Name & $\begin{array}{c}\text { Number } \\
\text { of Patches }\end{array}$ \\
\hline 1 & Northeast & 155 \\
\hline 2 & Mideast & 195 \\
\hline 3 & Northwest & 93 \\
\hline 4 & Midwest & 92 \\
\hline 5 & Central & 199 \\
\hline 6 & South & 131 \\
\hline
\end{tabular}

\section{Agents}

The system has five types of agents:

- Afghan government soldiers (denoted by $S$ ) 
- Coalition forces soldiers (denoted by $C$ )

- Taliban (denoted by $T$ )

- Leaders (denoted by $L$ )

- Citizen agents (denoted by $I$ ). Citizens who are supportive of the Taliban become Taliban helpers (denoted by $T H$ ), whereas citizens who are supportive of soldiers/coalition forces become soldier helpers (denoted by $\mathrm{SH}$ ). The rest are neutral and are denoted by $N$.

\section{Simulation Steps}

The following is a description of the simulation that tracks the process outlined in Figure 2-1, beginning at the Update step.

Figure 2-1. Conceptual flow chart of the simulation

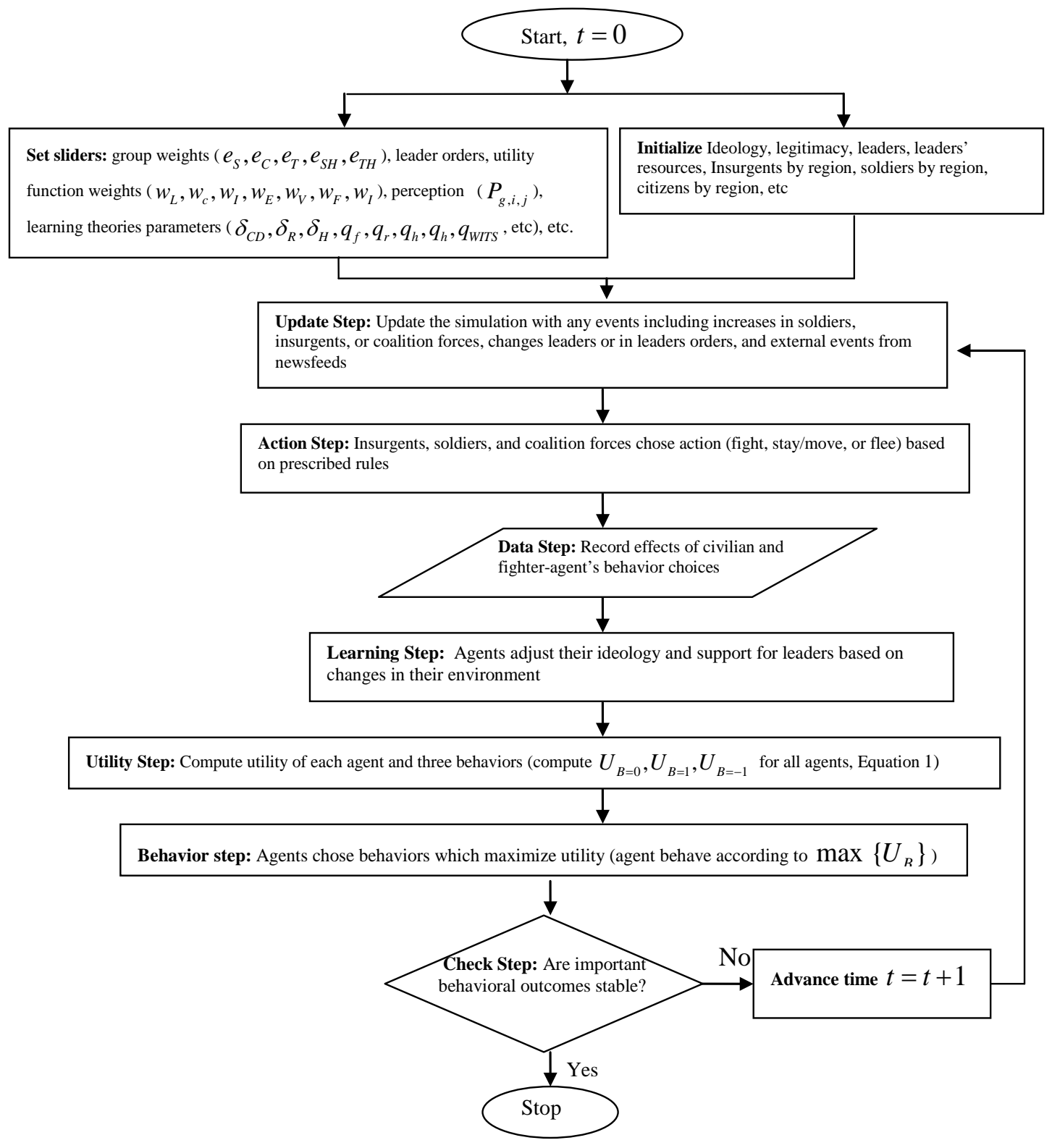




\section{Update Step}

In this step, all external events are inputted into the simulation. These events include important political, military, and economic events. Examples would include arrival or departure of some Afghanistan government forces (AGF), coalition forces (CF), or Taliban, the death of a leader, news that may inflame or pacify the public, etc. The events in this step may lead to changes in the leaders' orders or their resources, and the changes are then inputted into the system.

\section{Action Step}

This step simulates the actions of AGF, CF, and Taliban on each patch. These actions may be to fight, to make an organized move, to stay on the patch but avoid fighting, or to flee. The decision of each group is influenced by its own strength, its perception of the strength of the opposing forces, and by the degree of support the group has from the local population. Furthermore, each group is assigned a certain effectiveness that depends on the quality of the group's training, command and control, weapons, etc. A detailed description of this step follows.

Table 2-3. Agent ability to identify other agents

\begin{tabular}{|c|c|c|c|c|c|}
\hline \multirow[t]{2}{*}{ True Identity } & \multicolumn{5}{|c|}{ Perceived Identity } \\
\hline & $\begin{array}{l}\text { Coalition } \\
\text { Forces }\end{array}$ & $\begin{array}{l}\text { Soldier } \\
\text { Helper }\end{array}$ & Neutral & $\begin{array}{l}\text { Taliban } \\
\text { Helper }\end{array}$ & Taliban \\
\hline $\begin{array}{l}\text { Coalition } \\
\text { Forces }\end{array}$ & $P_{S, C, C}$ & $P_{S, C, S H}$ & $P_{S, C, N}$ & $P_{S, C, T H}$ & $P_{S, C, T}$ \\
\hline Soldier Helper & $P_{S, S H, C}$ & $P_{S, S H, S H}$ & $P_{S, S H, N}$ & $P_{S, S H, T H}$ & $P_{S, S H, T}$ \\
\hline Neutral & $P_{S, N, C}$ & $P_{S, N, S H}$ & $P_{S, N, N}$ & $P_{S, N, T H}$ & $P_{S, N, T}$ \\
\hline Taliban Helper & $P_{S, T H, C}$ & $P_{S, T H, S H}$ & $P_{S, T H, N}$ & $P_{S, T H, T H}$ & $P_{S, T H, T}$ \\
\hline Taliban & $P_{S, T, C}$ & $P_{S, T, S H}$ & $P_{S, T, N}$ & $P_{S, T, T H}$ & $P_{S, T, T}$ \\
\hline
\end{tabular}

Each group of agents is given the ability to identify other agents correctly or incorrectly as shown in Table 2-3, where $P_{g, i, j}$ is the percentage of individuals from group $i$ perceived to belong to group $j$ by individuals in group $g$, e.g., $P_{S, T, N}$ is the percentage of Taliban perceived to be neutral citizens by soldiers. For example, if there are 100 coalition forces on a patch (true identity), then those 100 agents can be perceived as coalition forces, soldier helpers, neutrals, etc. The five percentages in each row need not add up to exactly $100 \%$; if the sum is more than $100 \%$, then the perception is that the total is more than reality; less than $100 \%$ would mean some agents are not being perceived. We assume that each group, including soldiers, identifies its own members correctly $100 \%$ of the time. 
The percentages $P_{g, i, j}$ are set using a slider. Note that it is assumed Afghan government forces will always be correctly perceived by Afghan government forces, and these settings therefore do not have sliders.

Each group is assigned an effectiveness that is computed on the patch level. For example, the effectiveness of soldiers is computed at the patch level according to

$$
E S=\frac{e_{S} \times n_{S}+e_{C} \times n_{C}+e_{S H} \times n_{S H}}{e_{S} \times n_{S}+e_{C} \times n_{C}+e_{T} \times n_{T}+e_{T H} \times n_{T H}+e_{S H} \times w_{T H}} \in[0,1.0]
$$

where $e_{i}$ is the weight associated with agent group $i$. $e_{i}$ depends on many attributes of group $i$, including training, equipment, effectiveness of command and control, and ability to bring additional support to bear, etc. Future iterations may take into account "force multiplier" effects of lopsided numbers; however, for now this assumes a linear relationship of force effect to force size.

$n_{i}$ is the number of agents of group $i$ present on this patch. The weights are set for each group using a slider called "force weights." Force weights indicate the relative strength of each group.

The soldiers have a perceived effectiveness that other groups evaluate according to

$$
\begin{aligned}
& \text { Soldier-Force }=S F=e_{C} \times n_{C}+e_{S} \times n_{S}+e_{S H} \times n_{S H} \\
& \text { Taliban-Force }=T F=e_{T} \times n_{T}+e_{T H} \times n_{T H} \\
& \text { Perceived-Soldier-Force }=P S F=e_{C} \times n_{C}+e_{S} \times n_{S}+e_{S H} \times n_{S H}
\end{aligned}
$$

In plain terms, the action that either the soldiers or the Taliban choose depends on the perceived effectiveness of the opposing force relative to the known effectiveness of their own force. The coalition forces and the Afghanistan government forces always act together, i.e., they choose the same action. Therefore, the perceptions by these two groups of the number of Taliban are averaged together to determine perceived Taliban count (PTC below). PTC is then used to calculate the perceived Taliban force, i.e., the number of perceived Taliban times the force weight for the Taliban, plus the number of perceived Taliban helpers times the force weight for Taliban helpers.

Once the perceived Taliban force is calculated, the soldiers calculate their own soldier force, which is the number of CF and AGF times their force weights, plus the perceived number of soldier helpers (PSHC) times the SH force weight.

$$
\begin{aligned}
& \text { Temp }=\left(P_{S, S H, T} \times n_{S H}+P_{S, N, T} \times n_{N}+P_{S, T H, T} \times n_{T H}+P_{S, T, T} \times n_{T}\right) \\
& P T C_{0}=\left(P_{C, S H, T} \times n_{S H}+P_{C, N, T} \times n_{N}+P_{C, T H, T} \times n_{T H}+P_{C, T, T} \times n_{T}\right) \\
& P T C=\frac{P T C_{0}+T e m p}{2} \\
& P T H C=\left(P_{C, S H, T H} \times n_{S H}+P_{C, N, T H} \times n_{N}+P_{C, T H, T H} \times n_{T H}+P_{C, T, T H} \times n_{T}\right) \\
& \text { PSHC }=\left(P_{C, S H, S H} \times n_{S H}+P_{C, N, S H} \times n_{N}+P_{C, T H, S H} \times n_{T H}+P_{C, T, S H} \times n_{T}\right) \\
& \text { Perceived-Taliban-Force }=P T F=\left(e_{T} \times P T C+e_{T H} \times P T H C\right)
\end{aligned}
$$


Total-Agents-Count-Sum $=$ tac $=(S F+P T F)$

Perceived-Effectiveness-Soldier $=P E S=(S F-P T F) /$ tac

Once the soldiers calculate the soldier force and the Taliban force, then they compare these two numbers to get a perceived soldier effectiveness ("Perceived-EffectivenessSoldier") that will range from -1 to +1 .

The perceived soldiers' effectiveness is computed by subtracting the perceived Taliban force in the numerator instead of adding as was done to compute the effectiveness of soldiers. Because this is a perceived effectiveness, this measure is allowed to fall below zero to indicate that a group may perceive itself to be at a severe disadvantage. This differs from the calculation for real effectiveness, ES, which will range from 0 to 1.

In determining the perceived effectiveness of the Taliban, perceptions of the coalition forces and the Afghan government forces on a patch for Taliban are averaged; for Taliban helpers, neutrals, and soldier helpers, the perceptions of the coalition forces are used.

The soldiers choose a course of action based on the "Perceived-Effectiveness-Soldier." They choose one of three actions: flee, move/stay, or fight. The thresholds for these actions are set by the sliders labeled "action choice." Essentially, if things look bad for the soldiers, they'll choose to flee; if things look good, they will choose to fight; somewhere in the middle, and they will choose to move/stay. The soldiers (coalition forces and the Afghan government forces) make this choice together, even though their force multipliers and success rates may be different.

Once an action is chosen, the success rate of that action is based upon the "success rate" sliders for the coalition, Afghan, and Taliban forces, respectively. The "fight" success rate of the CF and AGF is normalized on the basis of relative populations; for move and flee, both the success rates and the determinations of outcome for these two factions are calculated independently.

In some cases, these success rates are modified, depending upon the combination of choices that the two sides make. For example, if the soldiers choose to fight and the Taliban choose to flee, it is assumed that the soldier's choice will impede the Taliban's choice; hence, a negative adjustment to the success rate. The amount of the adjustment is set by the slider labeled "action-modifier." In the matrices below, a '-' sign indicates a negative adjustment and a '+' sign indicates a positive adjustment. (Initiative is currently assigned at random.)

Soldiers (CF and AGF) and Taliban should have an additional choice between "move" (to an adjoining patch) or "stay" (on the current patch). However, we also thought that this choice should not be predicated on the danger level previously calculated. Therefore, the choice "move/stay" is further divided by the slider labeled "stay?" for each of these three combatant factions. Note that the choice between move and stay is no longer made by the CF and AGF agents combined, but rather separately for each. Also, the success rate is separate for each. Finally, "move" is distinct from "flee" in that all the agents 
attempt to move to the same randomly-chosen (neighboring) patch, rather than fleeing to any randomly-chosen (neighboring) patch.

Figure 2-2. Results of action choices

Soldiers Have Initiative
\begin{tabular}{|l|l|l|l|}
\hline TIS & flee & $\begin{array}{l}\text { move/ } \\
\text { stay }\end{array}$ & fight \\
\hline flee & AlA & AlB & A-IC+ \\
\hline $\begin{array}{l}\text { move/ } \\
\text { stay }\end{array}$ & BVA & BIB & C-IC \\
\hline fight & CIA & C-IB- & CIC \\
\hline
\end{tabular}

Taliban Have Initiative
\begin{tabular}{|l|l|l|l|}
\hline TIS & flee & $\begin{array}{l}\text { move/ } \\
\text { stay }\end{array}$ & fight \\
\hline flee & AIA & AIB & A-IC+ \\
\hline $\begin{array}{l}\text { move/ } \\
\text { stay }\end{array}$ & BVA & BIB & C-IC \\
\hline fight & CIA & C-IB- & CIC \\
\hline
\end{tabular}

The grids in Figure 2-2 indicate the results of the action choice for each side. $\mathrm{T}$ is the Taliban choice (left column and left side in each cell) and $S$ is the soldier choice (top row and right side of each cell). The top row and left column indicate the choice of each side, but the cells indicate the action; in two cases - both when the Taliban choose move/stay and the soldiers choose fight - the Taliban are forced to fight, but now with a negative adjustment to their success rate. For the inner cells: $\mathrm{A}=$ flee, $\mathrm{B}=$ move/stay, and $\mathrm{C}=$ fight. Both the Taliban and the AGF agents have similar sets of sliders for perception, and identical formulae for perception, perceived effectiveness, etc.

\section{Data Step}

The outcomes of the Action Step are recorded. This includes the casualties, movement of agents, ideology of agents, mindshare, and territorial control.

\section{Learning Step}

Each agent has a tribal affiliation, a location within Afghanistan, and an initial ideology. Afghan citizens may support one side or the other based on which behavior maximizes their utility given by Eqn (1). In this step, citizen agents adjust their ideology and loyalty to the leaders based on the latest developments on their patch, given their previous choices. This step incorporates the learning theories described in the main body of this report. Agents' beliefs and attitudes change depending on the information available and the situation on their patch, the consequences they experience, and the beliefs and attitudes of other agents around them. A detailed description of this step follows. Each tribe has one or more leaders. Larger tribes such as the Pashtuns have several leaders. Each leader has

- resources $\in[0,1]$ : 0 none, 1 maximum possible; set using a slider;

- orders: $-1=$ help Taliban, $0=$ be neutral, +1=help government/coalition forces; 
- ideology: currently, leaders' ideologies are reflected in their orders.

Initial ideology of citizens is in the range $[-1,+1]$ where $-1=$ fully support the Taliban and $+1=$ fully support the government. The ideology is initialized with a normal distribution around a given mean for the tribe or subtribal group (set by a series of sliders). Each agent is responsive to exactly one leader.

For all of the equations concerning psychological change (learning), boundaries are set at 0 and 1 for loyalty and at -1 and 1 for ideology. Change can occur to push an agent's values up to those boundaries at which point no further change in that direction (i.e., beyond the boundary) is possible. This is realistic. Loyalty and ideology have natural endpoints: complete loyalty or none; completely behind the government or completely behind the insurgency. And there are people, often many people, who are at the boundaries. Hence, change should be able to push an agent until the agent reaches a boundary, and then the agent can go no further. This is analogous to a physical process where a boundary (e.g., a wall) exists that is independent of the process (e.g., some force) causing movement toward the boundary.

\section{Change in Loyalty}

Let $L=$ loyalty of the citizen agent to the leader. $L$ is given by

$L=\eta|O-B| / 2$

$O=$ leader's order: $-1=$ help Taliban, $0=$ be neutral, $1=$ help government;

$B=$ agent's behavior: $-1=$ help Taliban, $0=$ be neutral, $1=$ help government;

$\eta=$ agent $i$ 's perception of her leader's legitimacy: minimum of 0 and maximum of 1 .

Agent $i$ 's perception of her leader's legitimacy $\left(\eta_{i}\right)$ changes at each time step according to

$$
\eta_{i}(t)=\frac{w_{\eta 1} \eta_{1, i}(t-1)+w_{\eta 2} \eta_{2, i}(t-1)+w_{\eta 3} \eta_{3, i}(t-1)+w_{\eta 4} \eta_{4, i}(t-1)}{w_{\eta 1}+w_{\eta 2}+w_{\eta 3}+w_{\eta 4}}
$$

where $w_{\eta i}$ are weights assigned to each of the variables below.

1. Cognitive dissonance effect (Festinger 1957): The agent changes her loyalty on the basis of the distance between her own behavior and the order of her leader. Thus, let $\delta_{C D}$ be the speed with which cognitive dissonance operates. Then

$$
\eta_{1, i}^{\prime}(t)=\eta_{i}(t-1)+\frac{\left[1-5|O-B| / 2+(O-B)^{2} / 2\right] \delta_{C D}}{2}
$$


The above equation pushes loyalty up by half of $\delta_{C D}$ if the agent does what her leader orders, down by half of $\delta_{C D}$ if she differs a little, and down by a whole $\delta_{C D}$ if she does the opposite of what her leader orders. $\delta_{C D}$ is set using a slider and $\delta_{C D} \in[0,1] . \eta_{1, i}(t)$ is then forced on the range $[0,+1]$ using

$$
\eta_{1, i}(t)= \begin{cases}+1 & \text { if } \eta_{1, i}^{\prime}(t)>1 \\ \eta_{1, i}^{\prime}(t) & \text { if } 0 \leq \eta_{1, i}^{\prime}(t) \leq 1 \\ 0 & \text { if } \eta_{1, i}^{\prime}(t)<0\end{cases}
$$

2. Results-based effect (Coleman 1990): If the agent's situation improves at a time step, the agent's loyalty to her leader increases, and the reverse. Thus, let $\delta_{R}$ be the speed with which results-based adjustment operates. Then

$$
\eta_{2, i}^{\prime}(t)=\eta_{i}(t-1)-\left[\left(H_{i}(t-1)-H_{i}(t-2)\right] \delta_{R}\right.
$$

where $H_{i}(t)$ is agent $i$ 's welfare, which is a combination of the economic welfare ( $E$, from the utility function equation) and the security threat ( $S$ ). Economic welfare is described below. The security threat $(S)$ is calculated in the following way, using the four components of the security factor $\left(r_{f}, r_{h}, r_{b}\right.$, and $\left.r_{c}\right)$ :

$$
r_{f}= \begin{cases}1.0 & \text { if there is conflict this step } \\ 0.5 & \text { otherwise }\end{cases}
$$

Clearly, the 0.5 constant could be changed to something else, as long as it remains between 0 and 1 .

$$
r_{h}=\tanh \left(\frac{1}{k_{h}} \cdot D_{t-1}\right)
$$

where $k_{h}$ is a constant that is currently set to 10 , and $D_{t-1}$ is the number of deaths resulting from fighting in the previous time step.

$$
r_{b}=\frac{S+I}{S+I+N}
$$

where $S$ is the number of Afghan and coalition forces, $I$ is the number of Taliban, and $N$ is the number of citizens on the patch.

$$
r_{c}=\frac{\text { foes }}{\text { friends }+ \text { foes }}
$$

The above are combined to compute 


$$
\begin{aligned}
& S_{B}=\left(1-r_{f}\right)^{q_{f}}\left(1-r_{h}\right)^{q_{h}}\left(1-r_{b}\right)^{q_{b}}\left(1-r_{c}\right)^{q_{c}} \\
& q_{f}+q_{h}+q_{b}+q_{c}=1 .
\end{aligned}
$$

Note: The real-time inputs from the WITS data can be substituted into the security threat $(S$ ) in one of two ways:

i) They can be substituted in for $r_{f}$ using the formula:

$$
r_{f}= \begin{cases}1.0 & \text { if there is WITS conflict effects this step } \\ 0.5 & \text { otherwise }\end{cases}
$$

ii) They can be substituted in for $r_{h}$ using the formula:

$$
r_{h}=\tanh \left(\frac{1}{k_{h}} \cdot D_{t-1}\right)
$$

The security threat components with the WITS data added is

$$
S_{B}=\left(1-r_{f}\right)^{q_{f}}\left(1-r_{h}\right)^{q_{h}}\left(1-r_{r}\right)^{q_{r}}\left(1-r_{c}\right)^{q_{c}}\left(1-r_{\text {WITS }}\right)^{q_{\text {WITS }}}
$$

To complete the substitution of the WITS data, the user should reduce the weight of either $q_{f}$ or $q_{h}$ to zero and set the weight for $q_{\text {WITS }}$ to $q_{\text {WITS }}>0$, such that

$$
q_{f}+q_{h}+q_{r}+q_{c}+q_{\text {WITS }}=1
$$

The WITS data have a larger temporal and spatial effect than other measures of security threat. The focal patch of the effect is determined by the geospatial coordinates of the real event. The intensity at the moment and place of maximum effect are determined by the input data. The effect has both a temporal and spatial decay away from the moment and place of impact and has a maximum radius of effect around the point of impact, as set by the user.

$H_{i}(t)$ is calculated at time $t$ as a ratio between the sliders for the economics and security weights, $w_{E}$ and $w_{S}$, such that

$$
H_{i}(t)=\frac{E(t) \times w_{E}+S(t) \times w_{S}}{w_{E}+w_{S}}
$$

$\delta_{R}$ is set using a slider and $\delta_{R} \in[0,1]$.

$\eta_{2, i}(t)$ is then forced on the range $[0,+1]$ using 


$$
\eta_{2, i}(t)= \begin{cases}+1 & \text { if } \eta_{2, i}^{\prime}(t)>1 \\ \eta_{2, i}^{\prime}(t) & \text { if } 0 \leq \eta_{2, i}^{\prime}(t) \leq 1 \\ 0 & \text { if } \eta_{2, i}^{\prime}(t)<0\end{cases}
$$

3. Homophily effect (Bourdieu 1984, Carley 1986): The agent's beliefs become more similar to the beliefs of agents like himself who are around him at each time step. Thus, let $\delta_{\mathrm{H}}$ be the speed with which homophily-based adjustment operates. Then

$$
\eta_{3, i}(t)=\eta_{i}(t-1)\left(1-\delta_{H}\right)+\bar{\eta}_{i}(t-1) \delta_{H}
$$

where $\bar{\eta}_{i}(t)$ is the mean loyalty to agent $i$ 's leader in the eyes of other followers in agent $i$ 's vicinity at time $t$.

$\delta_{H}$ is set using a slider and $\delta_{H} \in[0,1]$.

4. Socialization (St. Augustine, $4^{\text {th }}$ century): This is the default theory, i.e., no change in agent's loyalty.

$$
\eta_{4, i}(t)=\eta_{i}(t-1)
$$

\section{Change in Ideology}

Agent $i$ 's ideology changes over time according to the following:

$$
P_{i}(t)=\frac{w_{P 1} P_{1, i}(t-1)+w_{P 2} P_{2, i}(t-1)+w_{P 3} P_{3, i}(t-1)+w_{P 4} P_{4, i}(t-1)}{w_{P 1}+w_{P 2}+w_{P 3}+w_{P 4}}
$$

where $w_{P i}$ are weights assigned to each of the variables below.

1. Cognitive dissonance effect (Festinger 1957): The agent's ideology changes in the direction of her own behavior. Let $\gamma_{C D}$ be the speed with which cognitive dissonance operates. Then

$$
P_{1, i}^{\prime}(t)=P_{i}(t-1)+\frac{\left[\left(B_{i}(t-1)-P_{i}(t-1)\right] \gamma_{C D}\right.}{2}
$$

$\gamma_{C D}$ is set by a slider and $\gamma_{C D} \in[0,1] . P_{1, i}(t)$ is then forced on the range $[-1,+1]$ using 


$$
P_{1, i}(t)= \begin{cases}+1 & \text { if } P_{1, i}^{\prime}(t)>1 \\ P_{1, i}^{\prime}(t) & \text { if }-1 \leq P_{1, i}^{\prime}(t) \leq 1 \\ -1 & \text { if } P_{1, i}^{\prime}(t)<-1\end{cases}
$$

2. Results-based effect (Coleman 1990): If the agent's situation improves, the agent's ideology shifts toward whoever controls the territory. Thus, let $\gamma_{R}$ be the speed with which results-based adjustment operates. Then

$$
P_{2, i}(t)=P_{i}(t-1)+C(t-1)\left[\left(H_{i}(t-1)-H_{i}(t-2)\right] \gamma_{R}\right.
$$

where $H_{i}(t)$ is agent $i$ 's welfare at time $t$ and reflects economic welfare, $E$ (from the utility function equation), and the agent's security, $S$. Economic welfare is described below. $H_{i}(t)$ is calculated as a ratio between the sliders for economics and security weights, $w_{E}$ and $w_{S}$ :

$$
H_{i}(t)=\frac{E(t) \times w_{E}+S(t) \times w_{S}}{w_{E}+w_{S}}
$$

$C(t)$ denotes the faction in control of agent $i$ 's territory at time $t$ (with the usual coding: $-1=$ Taliban, 1 =government, intermediate values indicate some degree of contested control).

$\gamma_{R}$ is set using a slider and $\gamma_{R} \in[0,1] . P_{2, i}(t)$ is then forced on the range $[-1,+1]$ using

$$
P_{2, i}(t)= \begin{cases}+1 & \text { if } P_{2, i}^{\prime}(t)>1 \\ P_{2, i}^{\prime}(t) & \text { if }-1 \leq P_{1, i}^{\prime}(t) \leq 1 \\ -1 & \text { if } P_{2, i}^{\prime}(t)<-1\end{cases}
$$

3. Homophily effect (Bourdieu 1984, Carley 1986): The agent's beliefs become more like the beliefs of others like himself. Thus, let $\gamma_{H}$ be the speed with which homophily-based adjustment operates. Then

$$
P_{3, i}(t)=P_{i}(t-1)\left(1-\gamma_{H}\right)+\bar{P}_{i}(t-1) \gamma_{H}
$$

where $\bar{P}_{i}(t)$ is the mean ideology of agents in agent $i$ 's territory at time $t . \gamma_{H}$ is set using a slider and $\gamma_{H} \in[0,1]$.

4. Socialization (St. Augustine, $4^{\text {th }}$ century): This is the default theory, i.e., no change in adult agent's ideology. 


$$
P_{4, i}(t)=P_{i}(t-1)
$$

\section{Utility Step}

At this step, given the updated ideology and loyalty from the learning step together with the economic, coercion, violence, repression and social influence variables, each agent computes the relative utility of each of the three behavior choices he or she has: be neutral (0), help the AGF and CF (+1), or help the Taliban (-1). The utility function for citizen agents is given by the Cobb-Douglas function in Eqn (1). This function is evaluated three times at each time step for each agent, representing the three behavior choices each agent considers. A detailed description of this step follows:

$C$ = coercion experienced by the agent from the leader. $C$ is given by

$$
C=R|O-B| / 2
$$

where $R \in[0,1]$ is the relative magnitude of the resources that can be brought by the leader to bear against an agent. $R$ is set using a slider.

$I$ = force exerted by the difference in ideology between the agent and the course of action he is considering. $I$ is given by

$$
I=|P-B| / 2
$$

where $P \in[-1,+1]$ denotes the ideology of the agent , $-1=$ fully pro-Taliban, $+1=$ fully pro-government.

$E=$ economic welfare experienced by the citizen agent. $E$ is given by

$$
E=\left(E^{+}-E B\right) /\left(E^{+}-E^{-}\right)
$$

where $E^{+}$denotes the best possible feasible economic situation for an agent, $E^{-}$denotes the worst possible economic situation for an agent, and $E B$ denotes the economic situation the agent expects by following the given considered behavior. If the citizen agent belongs to an area where opium is grown (a 38\% chance on "opium" patches), then $E^{+}=\$ 2000$; if the citizen agent belongs to an area where opium is not grown, then $E^{+}=\$ 300$; and the worst economic situation all agents expect is $E^{-}=\$ 50$.

$V=$ violence welfare experienced by the citizen agent. $V$ is given by

$$
V=\frac{v_{c}}{v_{C}^{-}}
$$


where

$$
v_{c}=\frac{\text { foes }}{\text { friends }+ \text { foes }}
$$

$v_{c}^{-}$is the worst possible value of $v_{c}$.

Repression operates only if the citizens are helping the side that is outnumbered on their patch. If the considered behavior is neutrality or helping the predominant force on their patch, no repression occurs $(R=0)$. If repression occurs, it is less effective the more that other citizens in some circle around the deciding agents are defying repression. Thus, citizens are less likely to defy factions that have a relatively strong presence on their patch, especially when that faction has little support among other citizens in the general area. Let $R=$ effectiveness of repression by armed forces (Taliban or Afghan forces) at the patch level. $R$ is computed as follows:

$$
\kappa=\frac{n_{S}+n_{C}-n_{T}}{n_{S}+n_{C}+n_{T}+n_{I}}
$$

where

$n_{s}=$ the number of government soldier agents in the focal agent's patch, $n_{C}=$ the number of coalition soldier agents in the focal agent's patch, $n_{T}=$ the number of Taliban insurgent agents in the patch, and $n_{I}=$ the total number of citizen agents in the patch.

Positive values of $\kappa$ indicate that the AGF and CF outnumber the Taliban, whereas negative values indicate the opposite. The magnitude of $\kappa$ indicates the degree to which one faction outnumbers the other. $R$ is then given by

$$
R=\max [0,-\operatorname{Sign}(\kappa) \times \operatorname{Sign}(B)] * \max \left[\kappa^{2}-\frac{1}{2}(L B)_{t-1}^{2}, 0\right]
$$

where $L B=\frac{\text { Number of citizen agents taking action } B}{\text { Number of citizen agents }}$, the proportion of citizen agents in a larger geographical area (one or more patches) performing behavior $B$.

The first factor in Eqn (45) is 0 unless the considered behavior opposes the dominant force on the patch. The second factor increases with the predominance of the fighters of one side and decreases the more citizens in the given area are performing the behavior this particular agent is considering.

For example, suppose that for a given patch, $n_{S}=0, n_{C}=0, n_{T}=200, n_{I}=50$, and all citizens were neutral in the preceding time step. Then $\kappa=-200 / 250=-0.8$. For behavior $B=+1$ (support soldiers) 


$$
\begin{aligned}
R_{(B=+1)} & =\max [0,-\operatorname{Sign}(-0.80) \times \operatorname{Sign}(+1)]^{*} \max \left[(-0.80)^{2}-\frac{1}{2}\left(\frac{0}{50}\right)^{2}, 0\right] \\
& =\max [0,1]^{*} \max [0.64,0]=0.64
\end{aligned}
$$

Suppose 30 citizens support the Taliban. Then for behavior $B=-1$ (support Taliban)

$$
\begin{aligned}
R_{(B=-1)} & =\max [0,-\operatorname{Sign}(-0.80) \times \operatorname{Sign}(-1)] * \max \left[(-0.80)^{2}-\frac{1}{2}\left(\frac{-30}{50}\right)^{2}, 0\right] \\
& =\max [0,-1] * \max [0.46,0]=0
\end{aligned}
$$

For the above example, if $w_{R}=0.2$, for support soldiers $(1-R)^{w_{R}}=(1-0.64)^{0.2}=0.815$, whereas for support Taliban, $(1-R)^{w_{R}}=(1-0)^{0.2}=1$, which implies that for this patch, repression hinders support for soldiers but does not hinder support for the Taliban.

For the influence of close associates, a quantity represented by $(1-F)^{w_{F}}$ in the utility function, let $F_{B}=$ the deviation of an agent's behavior from the behavior of agents within a certain radius of the focal agent in the previous time step (i.e., $t-1)$. $F$ is computed as follows:

$$
F_{j}=\frac{1}{2}\left|B_{j}-\left(\frac{1}{n_{I}} \sum_{i \in n_{I}} B_{i}\right)_{t-1}\right|
$$

For example, if there are $n_{I}=400$ on a patch and 100 of them supported the soldiers last time and 20 supported the Taliban in the last time step, then for "support Taliban"

behavior $F_{B=-1}=\frac{1}{2}\left|-1-\frac{-20+100}{400}\right|=0.60$, for "neutral” behavior $F_{B=0}=\frac{1}{2}\left|0-\frac{-20+100}{400}\right|=0.10$,

and for "support the soldiers" behavior $F_{B=+1}=\frac{1}{2}\left|1-\frac{-20+100}{400}\right|=0.40$. If $w_{F}=0.2$, then for “support soldiers" $(1-F)^{w_{F}}=(1-0.6)^{0.2}=0.83$, for "neutral” $(1-0.1)^{0.2}=0.98$, whereas for "support Taliban," $(1-0.4)^{0.2}=0.90$, which implies that for this patch, influence works to increase support for soldiers relative to Taliban.

\section{Behavior Step}

In this step, each agent picks the behavior that maximizes his or her utility, which was computed in the Utility step. Every Taliban helper and soldier helper has a force weight that indicates his or her relative contribution to the battles. Therefore, this aggregate behavior of the citizen agents influences the subsequent action choices for the CF, AGF, and Taliban by affecting the perceived effectiveness for each. It also contributes to the real effectiveness of each, which is used to determine the Action step's outcomes. 


\section{Check Step}

In this step, the outputs from the simulation (i.e., territorial control, hardship, and mindshare) from the current step are compared to those from the previous steps to determine if all of the outcomes have become sufficiently stable to terminate the simulation. The length of the simulation run will depend on the frequency and the significance of the events and changes inputted in the Update step. If the purpose of a run is training and testing the relationship between simulation outputs and real-world events, then we are constrained to using the putative relationship between the simulated and realworld clock. In this case the stabilization criterion is no longer used because these experiments constrain the total number of simulated time steps that are allowed to elapse to represent the seven months that transpired real-world.

\section{NetLogo Simulation Outputs}

\section{Territorial Control}

This describes the control at the patch level and is computed as follows:

$$
\text { Control }=\frac{n_{S} \times e_{S}+n_{C} \times e_{C}-n_{T} \times e_{T}}{n_{S} \times e_{S}+n_{C} \times e_{C}+n_{T} \times e_{T}}
$$

where $n_{S} \times w_{S}+n_{C} \times w_{C}$ is the effectiveness of soldiers and $n_{T} \times w_{T}$ that of the Taliban.

\section{Hardship}

$$
\text { hardship }=\text { clip-to-range }_{0}^{1}\left(\frac{\text { security } \cdot \mathrm{V}+\text { economics } \cdot \mathrm{E}}{\text { security }+ \text { economics }}\right)
$$

Here clip-to-range is a "squashing" function used to keep hardship values between 0 and 1. Economics and security sliders range from 1 to 10 and can be used to select the relative importance of these two aspects of pressure that agents are facing.

\section{Mindshare}

Mindshare refers to which side the citizen agents support on the aggregate. It is calculated on the patch level based upon how many of the citizen agents choose to support the government, stay neutral, or support the Taliban. The aggregate of this attribute is used to track and measure certain regional effects. Mindshare that is heavily tilted one way or the other can also influence the outcome of combat. Mindshare is calculated as follows: 


$$
\text { Mindshare }=\frac{n_{\mathrm{SH}}-n_{\mathrm{TH}}}{n_{\mathrm{N}}+n_{\mathrm{SH}}+n_{\mathrm{TH}}}=\frac{n_{\mathrm{SH}}-n_{\mathrm{TH}}}{n_{I}}
$$

\section{The Simulation Scenario}

These specific simulations are based on the situation from 2005 to 2007 in the Ghazni region of Afghanistan, about halfway between Kabul and Kandahar. The region was chosen because of a high level of Taliban activity in that period and a counterinsurgency operation (Operation Maiwand) by the Afghan government and coalition forces in June 2007. The Taliban use removal and intimidation of the local leadership as a tactic to increase their control over the population. As one of our input variables, therefore, we use assassinations or attempted assassinations of local leaders. Our output variable will be the allegiance of the citizen population.

\section{Calibration}

The simulation is trained on data covering seven months in 2007 (3/15/2007 to $10 / 15 / 2007$, to avoid a seasonal effect in violence unaccounted for by our simulation). This year was selected because it contains a good variety of input data events on which to train the simulation.

The three significant components of the calibration process, in which key parameter values are tried and evaluated, are the inputs, the outputs, and the correlation measure between the simulation and the real-world data.

\section{Inputs}

The simulation accepts both static inputs and variable inputs. The static inputs are those that come directly from data, including the initial population settings as well as events that were known to transpire during the simulation period. The variable inputs are controls that we perturb during calibration to identify useful settings.

\section{Static Inputs}

The static inputs to the simulation are detailed in other sections of this document. Briefly, they include

- geography

- population per patch (including religious affiliations and tribal memberships)

- hand-selected political events (assassinations of prominent figures; see below); not all political events have direct analogues within the current simulation, but assassinations do, and all were incorporated into the event stream 
- perceptive acuity for both factions: this is the likelihood of each faction correctly identifying its own units and its opponents' units, expressed in a matrix of sliders within the NetLogo simulation

- effectiveness measures for both factions

- leaders' resources and standing orders (coarse allegiance)

- periodic infusion rates of new soldiers and Taliban

\section{Regarding Assassination}

These are the specific assassination events that are introduced into the simulation: ${ }^{1}$

- April 16 $6^{\text {th }}, 2007$ - In Andar, Ghazni, Afghanistan, assailants launched rockets near a high school, causing no injuries or damage. The attack targeted the governor of the province, who was attending a road construction inauguration event. No group claimed responsibility, although it was widely believed the Taliban was responsible.

- June $1^{\text {st }}, 2007$ - In Khwaja Umari, Ghazni, Afghanistan, armed assailants abducted the Chief of Gomal District from his house. The Taliban claimed responsibility.

- June $2^{\text {nd }}$, 2007 - In Angor Village, Jaghuri, Ghazni, Afghanistan, armed assailants stormed the house of a police chief then fired upon and killed five civilians. The Taliban claimed responsibility.

- August $3^{\text {rd }}, 2007$ - Near Ghazni, Ghazni, Afghanistan, assailants abducted a provincial government official. The Taliban claimed responsibility.

- September $3^{\text {rd }}$, 2007 - Near the Leewani Bazaar, in Qareh Bagh, Ghazni, Afghanistan, assailants kidnapped two civilian relatives of a female legislator. The Taliban claimed responsibility.

The simulation assumes that Taliban assassination of pro-government leaders will have the effect of moving the aggregate leadership toward a pro-Taliban stance. Each act of aggression by the Taliban against that leader will move the leader's order toward -1 and away from +1 .

Furthermore, the leader's order is adjusted according to which side (the Taliban or the government) controls the Ghazni patches. Control is measured using the number of, and the force weights for, CF, AGF, and Taliban, such that

$$
\begin{aligned}
& \text { Soldier-Force }=S F=e_{C} \times n_{C}+e_{S} \times n_{S}+e_{S H} \times n_{S H} \\
& \text { Taliban-Force }=T F=e_{T} \times n_{T}+e_{T H} \times n_{T H}
\end{aligned}
$$

\footnotetext{
${ }^{1}$ These descriptions are copied verbatim from the WITS database.
} 


$$
\text { Control }=\frac{\left(n_{s} \times e_{s}+n_{c} \times e_{c}-n_{T} \times e_{T}\right)}{\left(n_{s} \times e_{s}+n_{c} \times e_{c}+n_{T} \times e_{T}\right)}
$$

where the $n$ values are the number of different factions present, the $e$ values are the effectiveness of each faction, and the $\mathrm{S}, \mathrm{C}$, and $\mathrm{T}$ subscripts refer to the factions themselves.

The leader's order is adjusted by control using the following function:

$$
O_{t+1}=O_{t}+\left(1-O_{t}\right) \times \max \left(0, \text { Control }_{t}\right)
$$

To understand this equation and why Taliban control does not influence the order, think of the status quo ante as the baseline: the leadership's order with no assassinations by the Taliban and no government troop surge. Then the Taliban and the government each have an activity available to them to affect the order: the Taliban can assassinate and the government can initiate a troop surge. If the surge fails militarily, meaning that the government fails to achieve control, then the leadership's order should remain unaffected, that is, return to the presurge status quo. (The rationale that the Taliban somehow seem more like "winners" by that failure so the leadership should shift to them was not assumed, because a failed surge could just as easily make a larger surge likely, and the leadership would not become more pro-Taliban in that case.)

In other words, control in the area by the coalition forces can affect a leader's orders, but control by the Taliban does not. These equations are incorporated into the flow diagram in Table 2-4.

Table 2-4. Simulation procedures within the conceptual flow diagram

\begin{tabular}{|l|c|}
\hline Simulation Procedures & Conceptual Flow Diagram \\
\hline $\begin{array}{l}\text { 1) Regenerate new agents - add surge } \\
\text { Time decay of WITS violence per patch }\end{array}$ & $\begin{array}{r}\text { Start, } t=0 \\
\text { 1) Input new WITS data - input assassination data; } \\
\text { update leader's order }\end{array}$ \\
$\begin{array}{l}\text { Small chance of civilians moving to neighboring patch } \\
\text { Record fighter-agent deaths (previous turn deaths: } \\
\text { "violence-last-turn") }\end{array}$ & $\bullet \quad$ Initialize \\
$\begin{array}{l}\text { Calculate influence and repression for utility function } \\
\text { Calculate real and perceived effectiveness }\end{array}$ & $\bullet \quad$ Action step \\
$\begin{array}{l}\text { Commence battle, or troops may move if no battle } \\
\text { Record fighter-agent deaths (deaths this turn: } \\
\text { "violence-this-turn”) }\end{array}$ & $\bullet \quad$ 2) Data step \\
$\begin{array}{l}\text { 2) Store control, mindshare, and E for each patch } \\
\text { store control for Ghazni region; adjust leader's } \\
\text { order based on control }\end{array}$ & $\bullet \quad$ Learning step \\
Calculate partial security threat for learning theory & $\bullet \quad$ 3) Utility step \\
\hline
\end{tabular}




\begin{tabular}{|l|c|}
\hline $\begin{array}{l}\text { Run learning theory module, which affects agent's } \\
\text { support for leaders and ideology }\end{array}$ & 4) Behavior step \\
$\begin{array}{l}\text { 3) Calculate remaining factors for utility function - } \\
\text { use adjusted leader's order from above }\end{array}$ & $\begin{array}{l}\text { Advance time or } \\
\text { 4) Run utility function } \\
\text { Refresh and visualize effects }\end{array}$ \\
\hline
\end{tabular}

\section{Variable Inputs (Key Parameters)}

The variable inputs differ among the three approaches to the simulation described in the body of this document.

\section{Concerning Time and Scheduling}

Note that the time scale is one of the parameters we allow to vary over the runs. We hope to identify a setting that best contributes to the realism of the simulation output while making no claim that the resulting time scale is psychologically or sociologically motivated, only that it was useful.

Later in this document, we describe stabilization criteria that are used to terminate runs. These criteria are appropriate in the context described later, but here, we are training and testing the relationship between simulation outputs and real-world events, so we are constrained to using the putative relationship between the simulated and real-world clock. Hence, these experiments constrain the total number of simulated time steps (ticks) that are allowed to elapse, to represent the seven months that transpired real-world.

The political events are, therefore, scheduled to correspond to ticks on the simulation clock. Table 2-5 shows the exact time steps used for events in the model runs. This is just an example; clearly, the experiment includes more than seven events in the simulations.

Table 2-5. Model event time steps

\begin{tabular}{|l|c|c|c|c|c|c|c|}
\hline & Event $\# \mathbf{1}$ & $\mathbf{\# 2}$ & $\mathbf{\# 3}$ & Surge & $\mathbf{\# 4}$ & $\mathbf{\# 5}$ & End \\
\hline 3 days & 11 & 26 & 27 & 37 & 47 & 58 & 68 \\
\hline 7 days & 5 & 12 & 12 & 16 & 21 & 25 & 35 \\
\hline 14 days & 3 & 6 & 6 & 8 & 11 & 13 & 23 \\
\hline
\end{tabular}

\section{Outputs}

The critical output is the mindshare, or the net level of support that a region has for the coalition government. Briefly, mindshare is computed using the proportion 
of the general citizenry that is charged (helping either the Taliban or the coalition solders).

\section{Three Incremental Agent Designs}

We pursued three options for describing the agents in our simulation, with the overriding goal of finding a description that produces simulation results most predictive of the real Afghanistan. The specific goals were as follows:

1. Generate three agent descriptions

a. One description should have entirely static agents.

b. One description should support adaptation.

c. One description should represent free exploration.

2. Run these three scenarios.

3. Evaluate the results.

4. Recommend an agent-based modeling solution based on the preceding efforts.

The three agent descriptions were embodied in three approaches, each an independent prototype (system) but all sharing a common base infrastructure. Each approach built upon (added functionality to) the previous approach:

1. The first approach used fixed values of attributes that describe the agents and their environment and then evaluated how faithfully the results represented the real world.

2. The second approach began with what we perceived were reasonable initial attribute values, but the model could learn and adapt over time to adjust these values.

3. The third approach began with random attribute values and was calibrated by a distributed genetic algorithm (GA) to favor those values in simulations that more faithfully represented the real world.

Each of the next sections summarizes one of these approaches and how each approach was motivated, designed, implemented, run, and evaluated.

\section{Approach 1 - Fixed Attribute Values}

This was the most direct case of the three. The values for the important parameters were fixed for each run, the simulation was conducted, and then the results were compared to what was observed in the real Afghanistan. For all of these approaches, the benchmarking against the real Afghanistan was conducted using an assumption that the mindshare produced by the simulation should positively correlate with the number of terrorist 
incidents recorded in real Afghanistan as reported in the Worldwide Incidents Tracking System, or WITS, database $;^{2}$ see Appendix 2 for an expanded discussion on precisely how this correlation is built.

These are the key parameters we perturbed per fixed simulation run, including their discrete values:

- $\quad$ troop surge magnitude: 0, 50, 100, 200, 500 troops

- time scale: 3 days/tick, 7 days/tick, 14 days/tick

- leadership theories: legitimacy, coercion, representative

- influence: on, off

- repression: on, off

Troop surge is a parameter because this time period within 2007 included Operation Maiwand. Although the number of real coalition and Afghanistan government soldiers deployed is known, we experimented with different troop deployment settings to calibrate the model's understanding of how many effective simulated troops this represents. The three leadership theories were combined with influence and repression, which attenuate the utility-function weights for the leadership theories as described in the appendices.

We evaluated all 180 combinations of these settings and selected the combination of parameter values that did the best job of producing outputs that matched real-world observations. NB: “doing the best job” was identified across multiple runs from clustering of the results and identifying common themes within well-performing groups.

\section{Generalizing across Parameter Settings}

We wished to know which settings worked best for the five parameters that we varied. For each parameter, the entire 180-run set was divided into partitions on the basis of that parameter's values, and the correlation was averaged among all members of each partition (Table 2-6). Having previously analyzed the effect of the number of periods on the correlation, we divided the 215-day window into 109 periods, because that figure represents a compromise between achieving steady-state (by using more periods) and minimizing computational time. In our analysis, no loss of generality ensued from using only 109 periods.

\footnotetext{
${ }^{2}$ We use this assumption for two main reasons: (1) it has some intuitive appeal and (2) the project has no better data available for benchmarking the per-agent state-of-mind.
} 
Table 2-6. Simulation results by run

\begin{tabular}{|c|c|c|c|}
\hline Parameter & Setting & Mean & $\begin{array}{l}\text { Standard } \\
\text { Deviation }\end{array}$ \\
\hline influence & $\mathbf{0}$ & 0.0761 & 0.0735 \\
\hline influence & 1 & -0.0773 & 0.0929 \\
\hline repression & 1 & 0.0318 & 0.0465 \\
\hline repression & 0 & 0.0241 & 0.0974 \\
\hline $\begin{array}{l}\text { social } \\
\text { theory }\end{array}$ & $\mathbf{L}$ & 0.2426 & 0.0301 \\
\hline $\begin{array}{l}\text { social } \\
\text { theory }\end{array}$ & $\mathrm{C}$ & 0.0399 & 0.0911 \\
\hline $\begin{array}{l}\text { social } \\
\text { theory }\end{array}$ & $\mathrm{R}$ & -0.1962 & 0.0414 \\
\hline surge & $\mathbf{0}$ & 0.2158 & 0.0486 \\
\hline surge & 50 & 0.0958 & 0.0767 \\
\hline surge & 100 & 0.0753 & 0.0707 \\
\hline surge & 200 & -0.0196 & 0.0947 \\
\hline surge & 500 & -0.1244 & 0.1383 \\
\hline time scale & 3 & 0.0887 & 0.1155 \\
\hline time scale & 7 & -0.0397 & 0.0488 \\
\hline time scale & 14 & 0.0505 & 0.0712 \\
\hline
\end{tabular}

We also conducted an analysis to determine which parameter setting combinations produced means that were statistically significant (Table 2-7). ${ }^{3}$

Table 2-7. Statistically significant parameter settings

\begin{tabular}{|l|l|r|r|r|r|r|}
\hline \multicolumn{1}{|c|}{ Parameter } & \multicolumn{1}{c}{ Settings } & $90.0 \%$ & $95.0 \%$ & $99.0 \%$ & $99.5 \%$ & $99.9 \%$ \\
\hline repression & $(0,1)$ & No & No & No & No & No \\
\hline influence & $(0,1)$ & Yes & Yes & Yes & Yes & Yes \\
\hline surge & $(50,500)$ & Yes & Yes & Yes & Yes & Yes \\
\hline surge & $(50,200)$ & Yes & Yes & Yes & Yes & Yes \\
\hline surge & $(200,500)$ & Yes & Yes & Yes & Yes & Yes \\
\hline surge & $(100,500)$ & Yes & Yes & Yes & Yes & Yes \\
\hline surge & $(100,200)$ & Yes & Yes & Yes & Yes & Yes \\
\hline surge & $(0,500)$ & Yes & Yes & Yes & Yes & Yes \\
\hline surge & $(0,50)$ & Yes & Yes & Yes & Yes & Yes \\
\hline surge & $(0,200)$ & Yes & Yes & Yes & Yes & Yes \\
\hline surge & $(0,100)$ & Yes & Yes & Yes & Yes & Yes \\
\hline
\end{tabular}

\footnotetext{
${ }^{3}$ We used a two-tailed t-test that assumes unequal variance.
} 


\begin{tabular}{|l|l|r|r|r|r|r|}
\hline surge & $(50,100)$ & No & No & No & No & No \\
\hline time scale & $(7,14)$ & Yes & Yes & Yes & Yes & Yes \\
\hline time scale & $(3,7)$ & Yes & Yes & Yes & Yes & Yes \\
\hline time scale & $(3,14)$ & Yes & Yes & No & No & No \\
\hline social theory & $(\mathrm{L}, \mathrm{R})$ & Yes & Yes & Yes & Yes & Yes \\
\hline social theory & $(\mathrm{C}, \mathrm{R})$ & Yes & Yes & Yes & Yes & Yes \\
\hline social theory & $(\mathrm{C}, \mathrm{L})$ & Yes & Yes & Yes & Yes & Yes \\
\hline
\end{tabular}

These results suggest that the best class of parameter settings we can recommend from these 180 runs is as follows:

Table 2-8. Recommended parameter settings

\begin{tabular}{|l|r|}
\hline \multicolumn{1}{|c|}{ Parameter } & Setting \\
\hline influence & off \\
\hline social theory & legitimacy \\
\hline surge & 0 \\
\hline time scale & 3 \\
\hline
\end{tabular}

The value of repression does not appear to matter significantly in these examples. Interpreting these results is challenging, because the argument here is only that we can identify single parameter settings that tend to increase the mean correlation between simulated mindshare and the number of WITS incidents within Ghazni. Saying that "influence $=$ off $(0.0761+/-0.0735)$ performs better than influence $=$ on (-0.0773+/-0.0929)" does not make any claim about what the higher correlation means. We take it as given that a higher correlation is more representative of what occurred in the real world, but we make no statement about how to map results observed in the simulated environment to outcomes one would expect.

\section{Analysis}

Among the runs in this approach, the largest separation between values was in the social theory parameter. Legitimacy performed (on average) significantly better than did either coercion or representative. For the legitimacy theory, and only for this theory, the violence weight is set to zero, meaning that the leaders' orders have more weight in the utility function than does any other single variable. Once assassinations are factored in, the positive correlation between mindshare and the number of WITS incidents follows naturally.

$U(B)=(1-L)^{W_{L}(1-C)^{W_{C}}(1-I)^{W_{I}}(1-E)^{W_{E}}(1-V)^{W_{V}}(1-F)^{W_{F}}(1-R)^{W_{R}}}$

where L is loyalty, $\mathrm{C}$ is coercion, I is ideology, $\mathrm{E}$ is economics, $\mathrm{V}$ is violence, $\mathrm{F}$ is influence, and $\mathrm{R}$ is repression. With the current inputs to the simulation, only three variables in the utility function really matter for changing allegiance: 
loyalty (L), coercion (C), and violence (V). Ideology and economic situation do not vary. Collapsing these variables into a single constant allows us to write a degenerate form of the utility function:

$U(B)=K(1-L)^{W_{L}}(1-C)^{W_{C}(1-V)^{W_{V}}}$

Assassinations translate into effects primarily on the leadership's orders and secondarily on the leadership's resources and those affect choice through loyalty and coercion. Letting $|\mathrm{A}|$ be the number of assassinations that have occurred within the simulation, $\mathrm{R}$ be the amount of resources the leader can bring to bear, and $\mathrm{O}$ be the leader's current order:

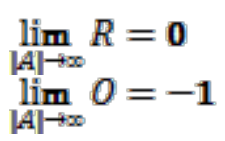

Rewriting the utility function, expanding the loyalty and coercion terms produces $U(B)=K\left(1-\frac{\eta|O-B|}{2}\right)^{\mathbf{W}_{\mathrm{L}}}\left(\mathbf{1}-\frac{\mathbf{R}|\mathbf{0}-\mathbf{B}|}{2}\right)^{\mathbf{w}_{\mathrm{C}}}(1-\mathrm{v})^{\mathbf{W}_{\mathrm{V}}}$

where $\eta$ is the degree to which agents perceive the leader to be legitimate. Because the factor weights sum to 1.0 by definition, decreasing $w_{\mathrm{V}}$ typically correlates with an increase in $w_{\mathrm{L}}$ and $w_{\mathrm{C}}$; thus, as the weight of violence goes down, the more the leadership's orders and resources matter. This suggests that, by the end of the simulation run (when the number of assassinations has plateaued), the utility function has become approximately

$U(B) \approx K\left(1-\frac{\eta \mathbf{l}-1-B \mid}{2}\right)^{\mathbf{w}_{\mathrm{L}}}(\mathbf{1}-\mathbf{v})^{\mathbf{w}_{\mathrm{V}}}$

Allowing $w_{\mathrm{V}}$ to approach zero makes this

$U(B) \approx K\left(1-\frac{\eta \mathrm{l}-1-B \mathrm{l}}{2}\right)^{\mathrm{w}_{\mathrm{L}}}$

This form is almost entirely dominated by the leader's order, which has become so close to -1.0 as to be indistinguishable from that value. This means that the utility function —once the violence weight approaches zero and a sufficient number of (WITS) assassinations has occurred-would be expected to produce mindshare outcomes that correlate positively with the number of WITS incidents, at least near the end of the simulated time period.

Note that the higher correlations generated when the violence weight is low are primarily artifacts of assumptions made about the data and their impacts. Specifically, we assumed that assassinations changed orders (i.e., a positive relationship existed). When the loyalty (and possibly coercion) weights are relatively high, the simulation produces a positive correlation between orders and allegiance as described above. Lastly, within the WITS data, other WITS 
incidents also correlate positively with assassinations, probably because both are aspects of terrorist violence. (We separated the two and used the former as inputs and the latter to evaluate outputs for purposes of calibration and sensitivity analysis.) We therefore would expect the positive correlation between WITS incidents and allegiance, especially when the violence weight is low.

\section{Approach 2 - Learning}

Once the initial, static system was complete, we sought to expand the dynamic range of the simulation by adding adaptation at the per-agent level. We did this by building a second prototype system that incorporated four theories of psychological change, or learning theories: cognitive dissonance, results-based, homophily, and socialization. The theories affect two agent attributes that enter into the utility function, namely support for the leadership and ideology. We present both the theory and the results from implementation of these theories within the NetLogo simulation.

\section{Learning Theory}

These theories add an important capability to the simulation for the social scientist or the operator on the ground who is interested in long-term changes and effects. The population's ideology and support for the leadership, and the ability to change these, have long been considered important operationally, as reflected in the goal of "winning hearts and minds." Reflecting their empirical importance, these two attributes are important in the leadership theories in the simulation model. Support for the leadership feeds into loyalty, a value that is especially important in the legitimacy theory. Ideology is emphasized in the representative theory.

From the theoretical side as well, it is valuable to add these theories of change in attributes. In the short term, it may be acceptable to take agents' attributes as fixed, but research in psychology and social psychology tells us that in the long run, attributes may change, especially psychological attributes such as attitudes. People's beliefs and attitudes may change because of information they receive and consequences they experience, as a result of the beliefs and attitudes of those close to them, and even as a consequence of their own behavior (Ajzen 2001, Petty et al. 1997). In fact, these effects have been shown specifically for the two attributes in question, support for leadership (Berger et al. 1998) and ideology (Nisbett and Ross 1980).

With respect to the two attributes, the theories operate identically although independently in the simulation, so we describe them only once. In each theory, each attribute has a separate weight parameter that the user sets to dictate the strength of its effect in the simulation. In addition, one parameter specifies the speed at which the change in support for the leadership can occur, and a parallel parameter specifies the speed for change in ideology. 
Cognitive dissonance theory (Festinger 1957, Nisbett and Ross 1980) posits that an individual resolves a contradiction between a behavior and a belief or attitude by changing the belief. In the simulation, therefore, this means that if an agent's behavior runs contrary to the leadership's order, the agent will lower his or her loyalty to, or support for, the leader; if the agent's behavior is in accord with the leadership's order, his or her support for the leader will increase. This is important to include in the simulation because the variety of values in the citizen agent's utility function may lead the agent to undertake a behavior that contradicts the leadership's orders, the agent's ideology, or both. Empirically, this certainly occurs as well.

The results-based theory (Coleman 1990) posits that an individual's beliefs and attitudes shift in response to how well he or she is doing. In the simulation, therefore, if an agent's situation improves, the agent's ideology shifts toward whichever side is in control of the agent's locality, and toward the opposite side if the agent's situation deteriorates. Likewise, the agent's support for the leadership increases if the agent's situation improves and decreases if it deteriorates. This matches the idea in the field of lowering unemployment and improving the local infrastructure to gain the loyalty and shift the ideology of the population.

The homophily theory (Bourdieu 1984, Carley 1986) posits that an individual's beliefs and attitudes become more similar to the beliefs and attitudes of those close to and similar to the individual. This is an attitudinal version of the effects of social influence seen for behavior in the social mobilization theories, and it has similar empirical support, such as attitudes in the former East Germany (Opp and Gern 1993). In the simulation, an agent shifts his or her support for the leadership toward the average support of similar others in the vicinity. Likewise, the agent shifts his ideology toward the average ideology of similar others in the vicinity.

Finally, the socialization theory posits that an individual's attitudes do not change in adulthood. Theoretically, this can be because attitudes are set in early adulthood, as proposed by the Jesuit motto, "Give me a child until he is seven, and I will give you the man," or because they are heritable (Olson et al. 2001), or both. In the simulation, this means that the agent does not change his or her support for the leadership and does not change his or her ideology. This theory can be combined meaningfully with another change theory to effect the idea that the support for the leadership or the ideology may be changeable, but only up to a point. This accords with empirical evidence that beliefs and ideologies are often stubborn and difficult to change.

\section{Implementation of Learning}

The learning theories were put in place to affect two key variables in the simulation: the support of the citizenry for their leaders, and the change in the ideology of the general populace. Each of these influences has the same general form: 
$v_{t+1}=v_{t}+\delta \Delta$

That is, the value at time $t+1$ is equal to the value at time $t$ plus some mitigated change (the lower-case delta is the maximum change allowable per one time step; the upper-case delta is the recommended change from the learning theory).

\section{Results from Implementing Learning}

Because both support and ideology share similar (high-level) functional form, testing these two learning theories was most easily accomplished by varying the value for $\delta$, the maximum allowable change resulting from learning in any single time step (Table 2-9). When $\delta=0$, the learning theories are effectively turned off, and the simulation degenerates into the system presented as Approach 1 in the preceding section.

Table 2-9. The additional evaluation series

\begin{tabular}{c|c}
$\delta_{\text {support }}$ & $\delta_{\text {ideology }}$ \\
\hline 0.0 & 0.2 \\
0.2 & 0.0 \\
0.2 & 0.2
\end{tabular}

Additional experimentation-not documented here-led us to the conclusion that delta values greater than 0.2 did not provide any additional benefit, as larger values often made the simulation too volatile to be useful. The results of varying these parameters across multiple replications are reported in Figure 2-3.

Figure 2-3. The results of varying ideology and leadership support parameters across multiple replications: (a) $\delta_{\text {support }}=0, \delta_{\text {ideology }}=0.2$; (b) $\delta_{\text {support }}=0.2, \delta_{\text {ideology }}$ $=0$; (c) $\delta_{\text {support }}=0.2, \delta_{\text {ideology }}=0.2$. 
(a)

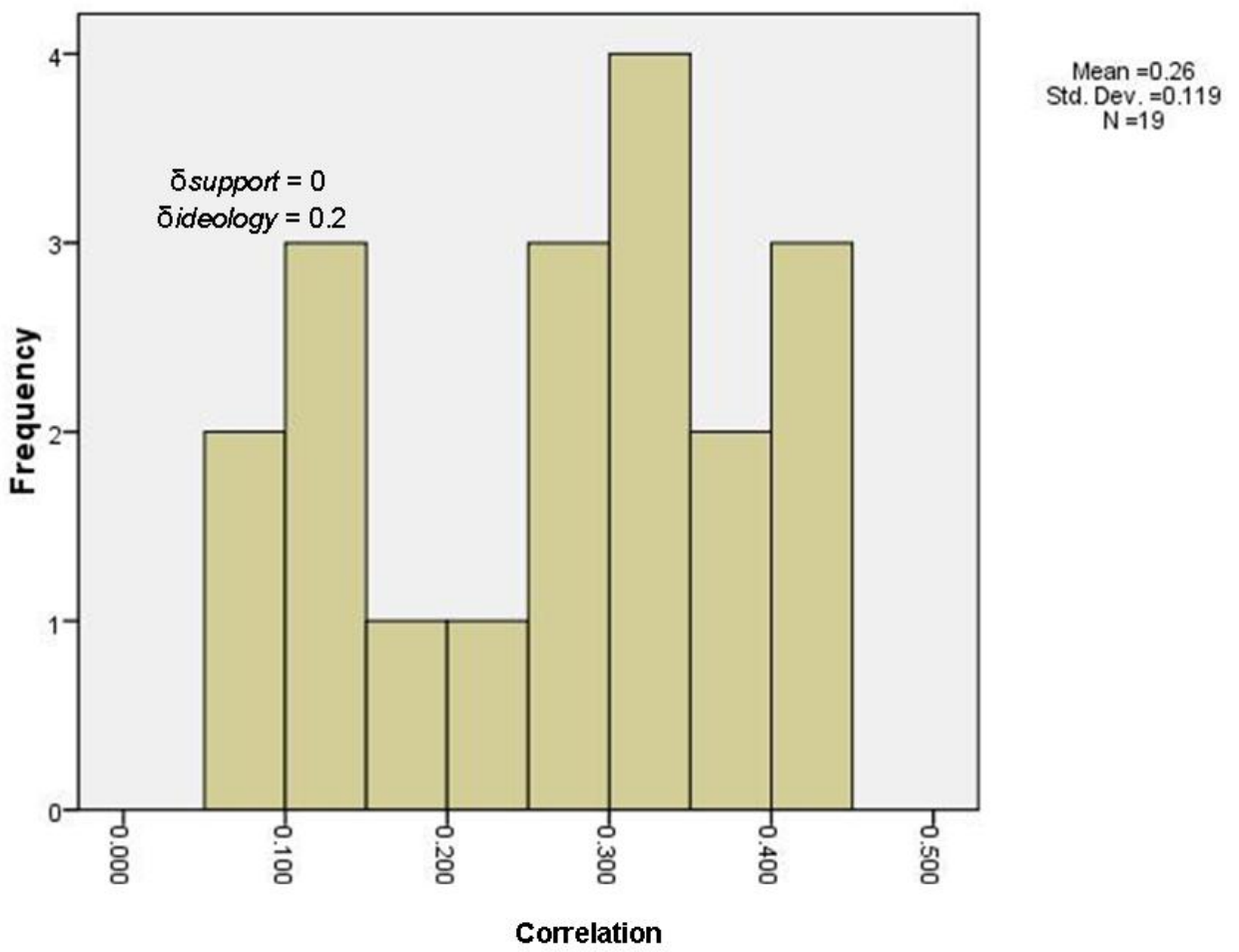

(b)

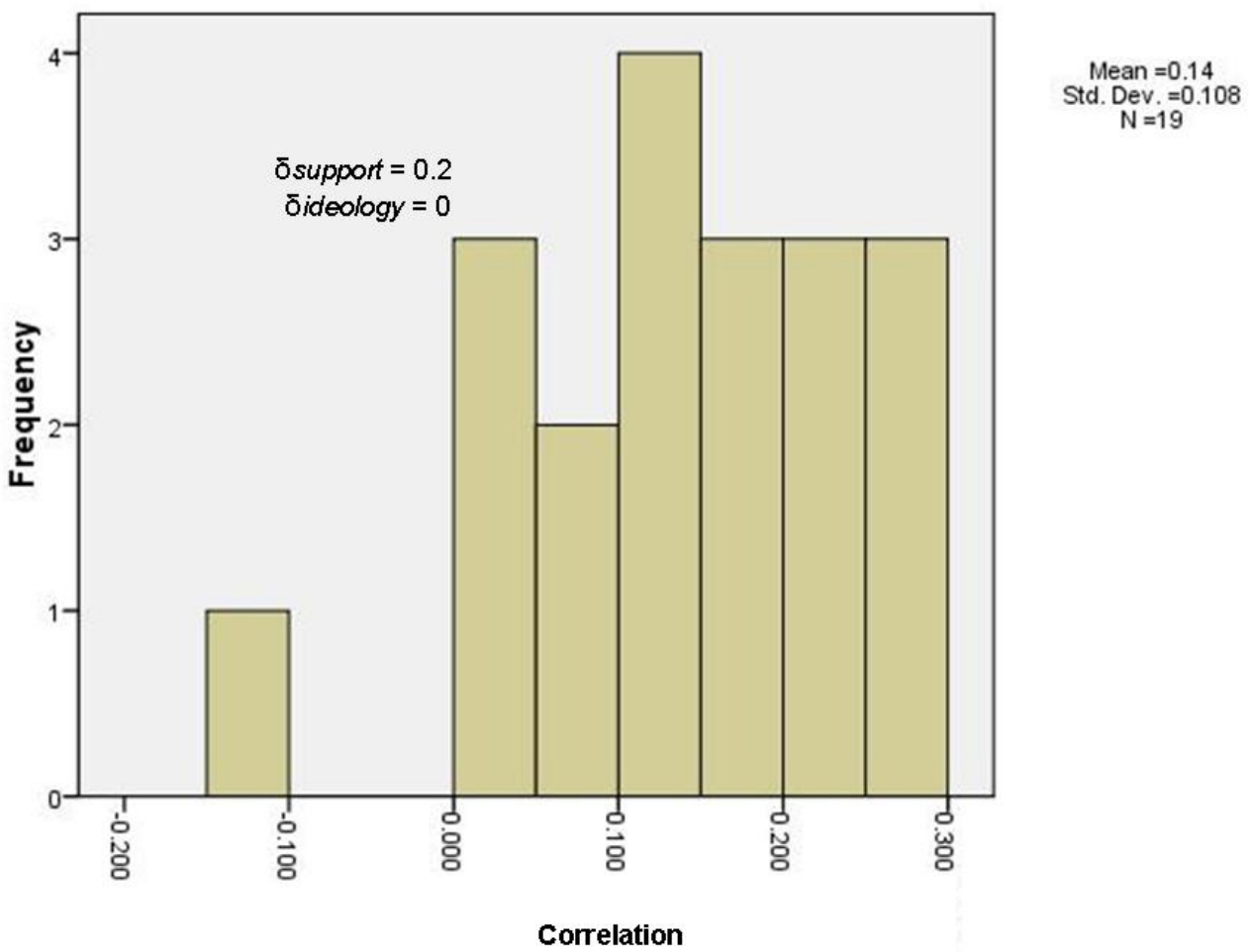


(c)

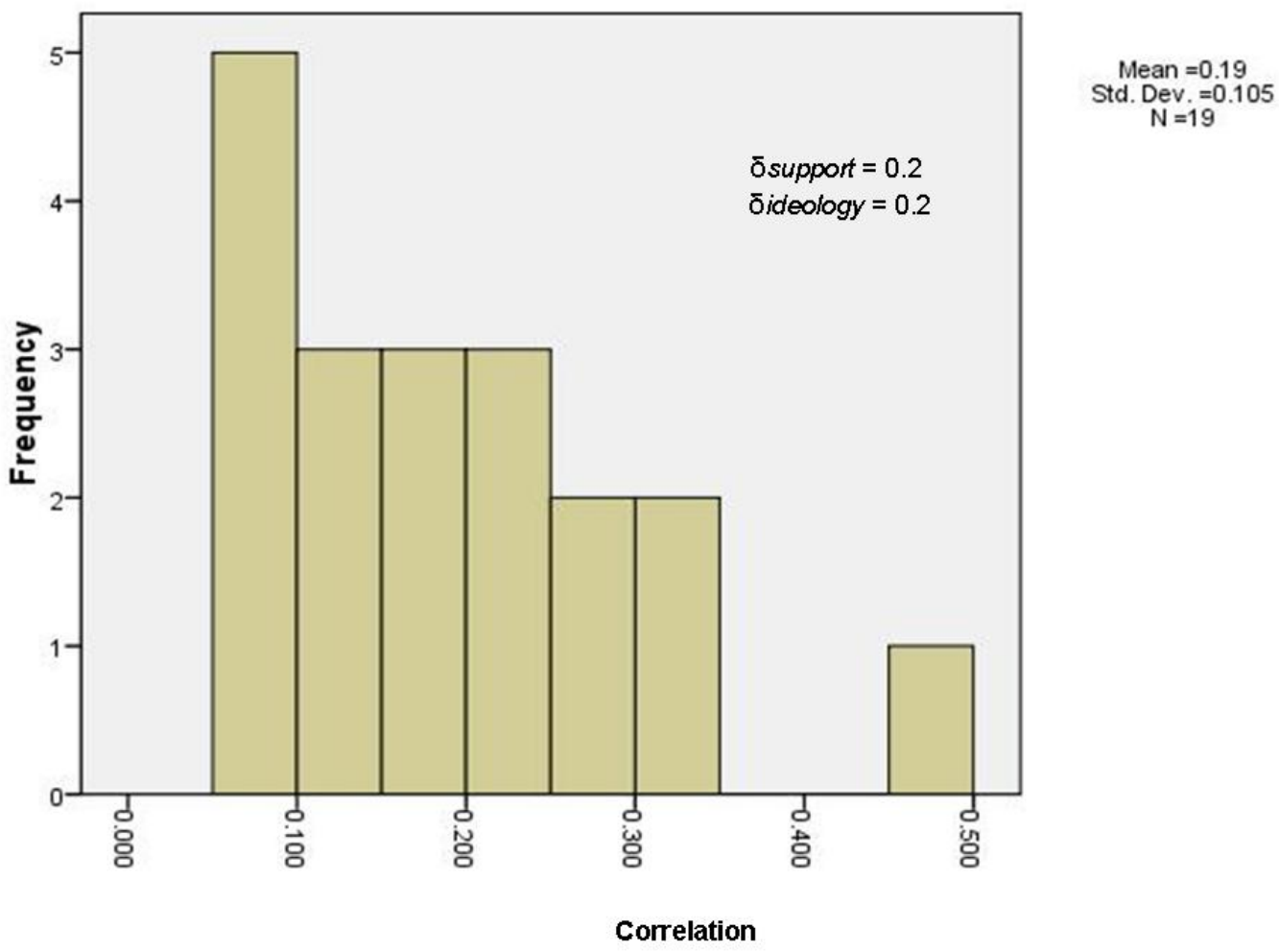

These plots suggest that the model may have slightly more expressive power when ideology - but not support for the leader-is allowed to change according to the learning theories (adaptation). It is important to note, however, that these three distributions are not significantly different from one another.

\section{Analysis}

Both support (loyalty) and ideology are top-level terms in the utility function, and both have four separate influences: cognitive dissonance; results-based effect; homophily; and socialization. Two of these theories have different impacts on the two variables:

1. Cognitive dissonance

a. updates support by moving the citizen in closer agreement with his leader

b. updates ideology by adding a momentum term to the agent's own behaviors

2. The results-based effect 
a. updates support on the basis of whether violence is nearby

b. updates ideology using only terms unrelated to violence, but rooted in our definitions of agent-based welfare

Homophily and socialization share direction and influence between support for leadership and changes in ideology, and hence should not contribute to the observed difference in mean correlation observed here.

The differences in the update procedures suggest a possible reason for the difference in mean correlation: because violence is sporadic in the simulations, its influence on support will also be sporadic; ideology is free of this chaotic influence. Additionally, updating ideology based on the agent's own momentum provides for yet another more consistent driver of the agent's behavior. These two effects combined may allow the agents to pursue a more consistent course of action with respect to ideology than with respect to support for leadership, providing a better foundation for the positive correlation.

\section{Approach 3 - Calibration via a Genetic Algorithm}

After completing the first two systems, the team moved on to the third and most flexible prototype system. In this approach, we chose to free a number of the simulation parameters, specifically those related to the social theories of leadership, and to allow a free search of the space by a distributed genetic algorithm (GA). We will review the technical design, the results and analysis, and directions for future work.

\section{Technical Design of the GA}

The core technical challenge is that the NetLogo platform on which the simulation is based is decidedly single-threaded, which stymies parallelism. To work around this, we constructed our GA system so that each processing node runs exactly one instance of the NetLogo simulation (in headless mode; see the NetLogo documentation at http://ccl.northwestern.edu/netlogo/docs/). A master node is always responsible for managing the central distribution of work and collecting results from the processing nodes. We dedicated three 8-core machines to the GA, running principally at night and during off-hours.

Because NetLogo is a Java application, we used a Java-based distribution framework for managing the GA: The Distributed Parallel Programming Environment for Java from IBM Alphaworks

(http://www.alphaworks.ibm.com/tech/dppej). This saved considerable effort, because we were able to merely subclass elements that had already been defined to manage the parallelization.

The specifics of the GA are as follows: 
1. The population size was dictated by the number of processing nodes available, typically between 16 and 24 chromosomes (at one chromosome per processing node).

2. Generations were synchronized by the master node.

3. Each chromosome was a vector of social theory (leadership) parameter values. Upon evaluation, each chromosome was instantiated in a headless instance of NetLogo, the simulation was run for the requisite 64 time steps, and the correlation between mindshare and WITS incidents was computed and returned—normalized to $(1+\mathrm{CORR}) / 2$ —as the fitness value.

4. When computing the next generation, we used single-point crossover (80\%) and simple Gaussian mutation (20\%).

5. The GA was stopped after completion of 100 generations; the members of the final generation were reported as the GA results, along with their fitness values.

6. A single run required almost 13 hours of clock time using 24 parallel computing nodes.

\section{Results from the GA}

After a number of trials, we found that individuals like the one reported below are representative of the best-performing chromosomes discovered by the GA:

Table 2-10. Best performing chromosomes

\begin{tabular}{|l|r|}
\hline Loyalty weight & 0.346 \\
\hline Coercion weight & 0.249 \\
\hline Ideology weight & $<0.001$ \\
\hline Economics weight & 0.096 \\
\hline Violence weight & 0.000 \\
\hline Influence weight & 0.167 \\
\hline Repression weight & 0.141 \\
\hline Correlation & 0.152 \\
\hline Fitness $(1+$ CORR)/2 & 0.576 \\
\hline
\end{tabular}

The course of the simulation is expressed in the following two charts (Figure 2-4) taken directly from the NetLogo instance. 
Figure 2-4. Course of simulations
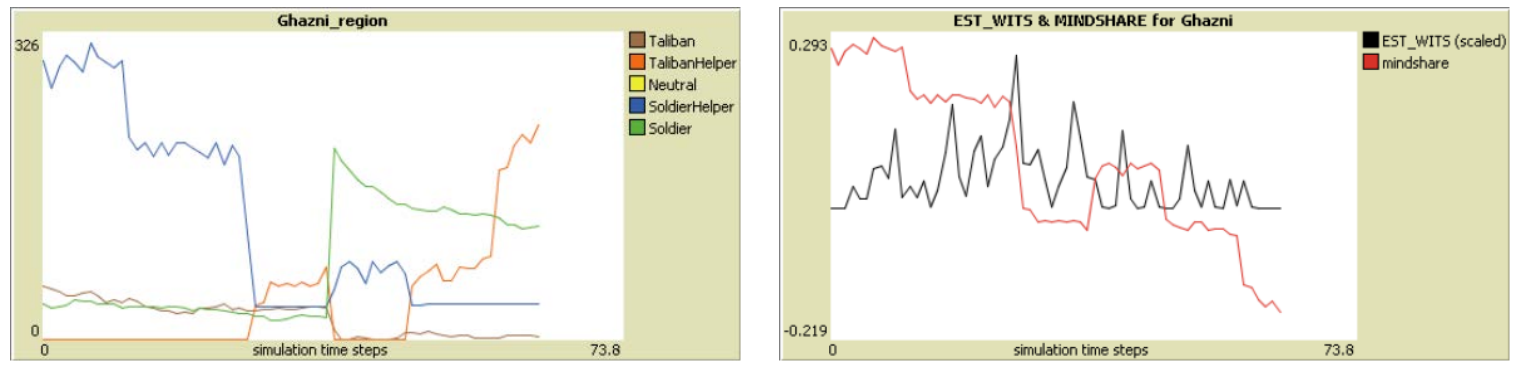

The left chart displays the composition of the Ghazni population over time. Most notable in this chart is the surge of soldiers corresponding to operation Maiwand. The right chart displays the two series we are trying to correlate: mindshare and the number of WITS incidents within Ghazni.

The most significant finding is that the weights for both ideology and violence are essentially zero. This is consistent with our findings in Approach 1, in which the legitimacy theory of leadership was identified as the best (average) performer; the legitimacy theory also specifies that the violence weight should be zero.

Furthermore, even though the correlation between mindshare and the number of WITS incidents is low (0.152), the correlation between mindshare and the leader's orders is high (0.977), again consistent with our hypothesis-developed within the analysis of Approach 1, above-that this is an artifact both of the nature of the model that is embedded within the simulation as well as the data with which we are testing the model.

It is interesting to note the difference between mindshare and control in the simulation for this winning chromosome. Consider these two images directly from the NetLogo simulation (Figure 2-5). 
Figure 2-5. The difference between mindshare and control in the simulation

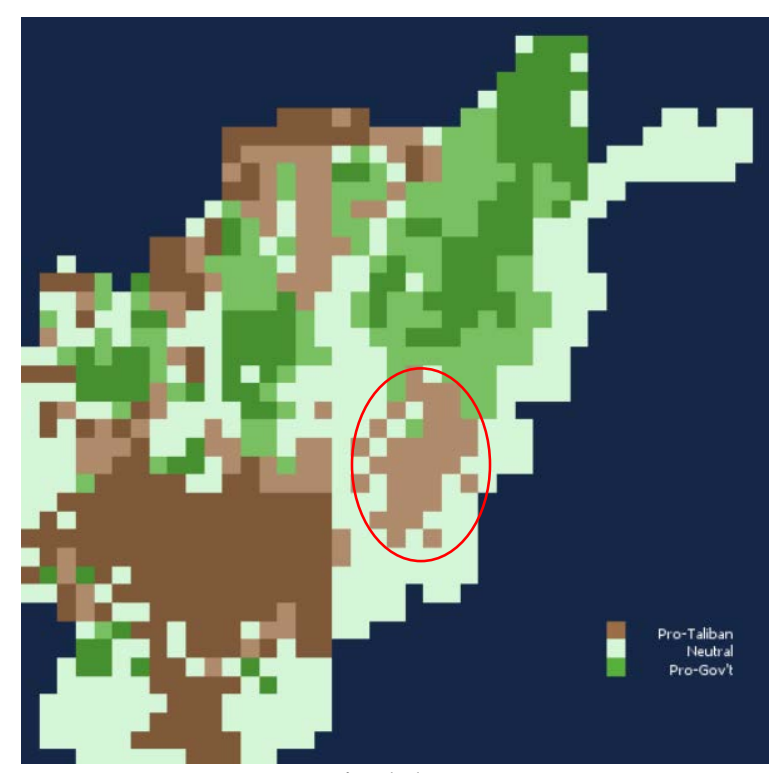

Mindshare

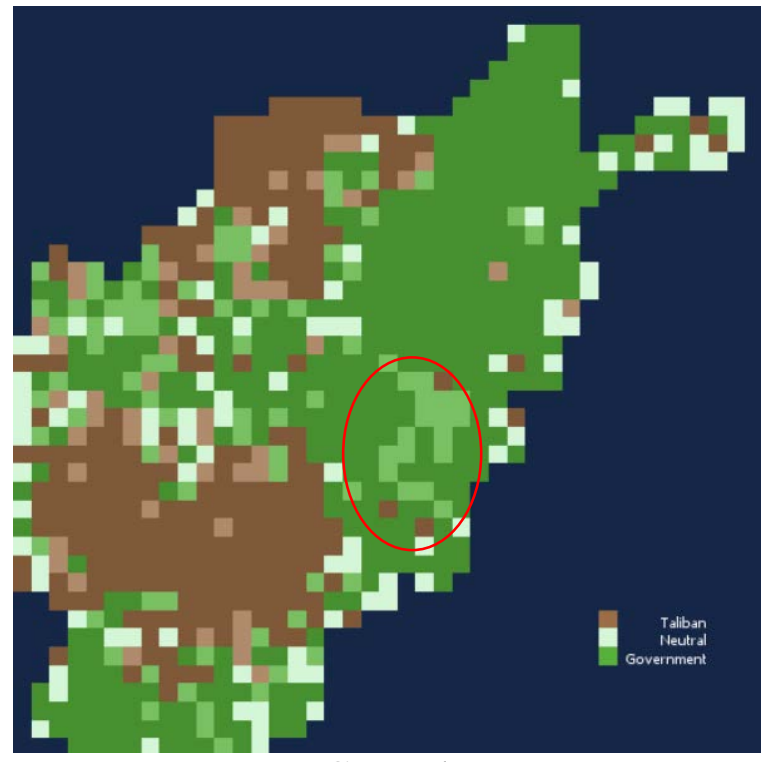

Control

Ghazni, loosely represented by the red oval superimposed on the two images, has been strongly influenced toward a pro-Taliban mindshare, yet it remains firmly in control of the coalition and pro-Afghan government.

We also conducted, but do not report here, additional work in which we ran the GA assuming that simulated mindshare and the number of WITS incidents were anti-correlated. Expectedly, a different set of parameter values bubbled to the top of the list of most-fit chromosomes. Of greater interest, though, was the fact that those runs also highlighted effects on mindshare resulting from the Maiwand surge that were not visible when the correlation was presumed to be positive: when the troops surged, the populace responded by rallying in a pro-government manner.

\section{Analysis of the GA}

The GA demonstrated the following advantages over both Approach 1 (static, predefined test cases) and Approach 2 (static cases with learning/adaptation):

1. The combinations of parameter values that we explored were significantly more varied than those we had hand-selected for Approach 1. Flexibility in choosing parameter combinations is especially important in a search algorithm operating in a search space as significantly multimodal as this simulated Afghanistan. Adaptation succeeds best only when it can overcome the exceedingly difficult problem of avoiding local minima, but without knowing the exact bounds on solution quality and volatility, a grid-based search is not appropriate. 
It is not reasonable to claim that the GA does any better job of recreating ground truth as observed in the real-world Afghanistan than does the set of discrete, static tests employed in Approach 1. Instead, the claim is simply that, not knowing precisely how well these simulations reproduce what happened in the real world, we find an advantage within the GA implementation because the technique is independent of the arbitrary constraints that underlie the selection of discrete simulations tested in Approach 1. The GA, then, is subject to far fewer social theory assumptions than is either of the preceding approaches.

While it is certainly possible that this flexibility is not required for this specific simulation, especially if there is some innate resilience within the simulation to change in light of varied parameter settings, the generality of the approach has merit.

2. Even though the parameter values were unconstrained, as mentioned in the previous point, the results we achieved were consistent with the conclusions drawn in Approaches 1 and 2: the simulation performs most successfully when violence weight is low.

3. Although the number of potential candidates (chromosomes) to evaluate is much higher with a GA than with either of the other two approaches described in this document, the confidence we have in the corroborating results among the three methods makes this increased computational effort worthwhile.

\section{Recommendations}

Because of the properties listed above, this third approach — in which a rule-based agent system adapts to extend its own behaviors, and yet can explore a more varied search space in a manner that produces findings that are consistent with our spot-checking of discrete cases - should allow us to conduct a more resilient investigation across the parameter search space within social science simulations.

\section{Future Work for GA}

Our work leads to the following conclusions and recommendations:

1. NetLogo was initially selected for our simulation platform, because it is easy for noncomputer scientists to use effectively. Unfortunately, being single-threaded is a significant drawback. Though we were able to overcome this difficulty, we strongly recommend using an alternate, more parallel-friendly agent-based simulation platform.

2. The top performers after 100 generations did not yield very significant correlation values. Many more of the chromosomes, even in the final population, produced negative correlations instead of positive ones. Combined with the previous analysis that showed more favorable simulation results when the violence weight 
is low, this suggests that the data we are using to calibrate/train the simulations may not be well suited for this task. It does not, however, reflect poorly on the calibration method itself.

3. A larger number of chromosomes should be evaluated. The 2400 we tested were too few to feel confident that we have explored a significant portion of the search space. Significantly greater computing power will be required to do this.

Computing the correlation between mindshare and WITS incidents within the NetLogo simulation, although convenient, was probably not the most effective way to gauge performance. Instead, the database we have put in place to capture NetLogo simulation data per tick should be used as a standard platform for performing the interpolation and aggregation of data required to assemble the correlation statistics. Unfortunately, this requires much deeper integration between the components.

\section{Insights}

This task offered several insights and key lessons learned:

- It is possible - even within a simulation that relies heavily on stochastic processes - to evaluate multiple replications of discrete combinations of parameter values to identify optimal simulation settings and trends.

- The simulation can exploit the underlying social models. This is a direct consequence of the equations embodying the social models, rather than an emergent behavior of the society of agents.

- Adaptation can increase the verisimilitude of the running model in some cases.

- The performed comparisons suggest that the third (genetic algorithm) approach to agent behavior performed the best: it explored the most granular search space, and produced results that were entirely consistent with earlier findings. 


\section{Chapter 3: Real-Time Updating}

\section{Introduction}

Simulations can be given several sets of alternative data, thereby enabling what-if analysis. A sufficiently robust simulation might actually be able to predict certain events, or learn to predict events over time (though such a capability is well beyond the scope of the initial project). However, the universe of potential outcomes must be constrained by evolving real-world events and as such, is limited to the universe of realistic options.

To incorporate these real-world events, a method was needed to collect data, transform it to the simulation semantics, and insert it as an event at the appropriate time in the simulation. For agent-based simulations, this implies that each of the agents would need to be stopped at the appropriate simulation time to allow for the insertion of the event. The techniques for managing the execution of simulation processes are well understood. We explain these techniques next and then apply them to agent-based simulation. Later in the report, we review the mechanisms available for harvesting external data and using them as simulation events.

\section{Computer Simulation Systems}

In a broad sense, we can divide computer-based simulation systems into two general classes. The first class, which is frequently called analytic simulation, intends the solution to a problem or experiment. Distribution of the simulation processes is a means to address scalability or performance issues of monolithic sequential simulations. In agent-based simulations, the simulation processes correspond to atomic units of composition. The natural decomposition of the simulation into groups of agents is an effective means of distributing the computation across processors. This, however, implies that the simulation processors will need to coordinate their progress so that the agents' processes are synchronized.

The second class of systems, which are called interactive simulations, embraces virtual reality, training simulators, and computer games. As one might expect, a major emphasis in distributed interactive simulation (DIS) is on creating a compelling, realistic, or entertaining distributed virtual environment (DVE) for human interaction. A relatively recent topic in interactive simulation is the melding of live, virtual, and constructive (LVC) objects into a blended (so-called “seamless”) environment.

The differences in the design priorities of the two classes of simulation directly influenced the development of message-passing protocols used to exchange data during a simulation execution. For example, a natural result of the analytic simulation perspective was a straightforward definition of correctness based on the answer provided by a sequential simulation execution, such as is done in our NetLogo simulations. Using this definition, regulating the delivery order of simulation messages is an important primary concern. Indeed, NetLogo implements a mechanism that ensures the agents stay in lock step (at the same tick) at all times. In contrast, the development of distributed virtual environments was not encumbered by such a strict notion of computational correctness. 
The overriding performance objective was providing real-time interaction for the users. It was convenient for designers of these systems to largely ignore the ordering of messages in favor of binding simulation time to the real-world (wall-clock) time.

\section{Analytic Simulation}

The first concepts of distributed analytic simulation appear in literature in the mid-1970s (Chandy and Misra 1978). The foundational research by Chandy and Misra focused on a distributed systems perspective of simulation (Chandy and Misra 1979). They presented concepts that paralleled the traditional notions of correctness for distributed computation. A fundamental test of a distributed computation algorithm was comparison to a sequential computation. Thus, a distributed simulation could be considered correct if its results were equivalent to those produced by a sequential execution of the same simulation. Synchronization techniques were designed to ensure sequential equivalence.

While preliminary notions of distributed simulation were being developed, additional models of message-passing distributed systems (not necessarily simulations) were evolving. Partial-ordering concepts, such as "happens-before" and "causality" (Lamport 1978), increased the asynchrony possible in an execution by removing certain constraints on delivery order. Using these concepts, several paradigms evolved as methods for ordering messages during an execution. The term time-management refers to general ways in which simulation processes coordinate message delivery to provide a correctly ordered distributed execution. Fujimoto provides an excellent overview of the evolution of parallel and distributed simulation and explains the important classes of timemanagement algorithms (Fujimoto 1990).

\section{Interactive Simulation}

Quite apart from the development of analytic simulations, a different form of distributed simulation was developed to enhance the quality of human-computer interaction. Military efforts in distributed interactive simulation were primarily a means of extending the usefulness of training simulators. (More about the military role in the development of distributed simulation is explained later.) Concurrent with the military-sponsored training initiatives, the computer-graphics community was extending the concepts of virtual reality to include multiple participants. The first articles describing distributed virtual environments appeared in the early 1990s, notably at the 1993 IEEE Virtual Reality Annual Symposium. Several independent efforts pursued a variety of architectural alternatives including client-server (Signh et al. 1994, Funkhouser 1995), peer-to-peer (Carlsson and Hagsand 1993), and independently replicated environment data (Macedonia at al. 1994). Overviews of (1) early development and (2) various paradigms of distributed virtual environments were provided by Stytz (1996) and Macedonia (Macedonia and Zyda 1997), respectively.

In these forms of distributed simulation, the simulation processes provide a means of real-time interaction with users (or user applications). Consequently, suspending a simulation execution is undesirable and frequently impossible. This highlights a fundamental difference between analytic and interactive simulation. Whereas analytic simulations may enforce strict message order constraints, many real-time simulations do 
Table 3-1. Analytic and interactive execution models

\section{$\underline{\text { Time-Managed Execution }}$}

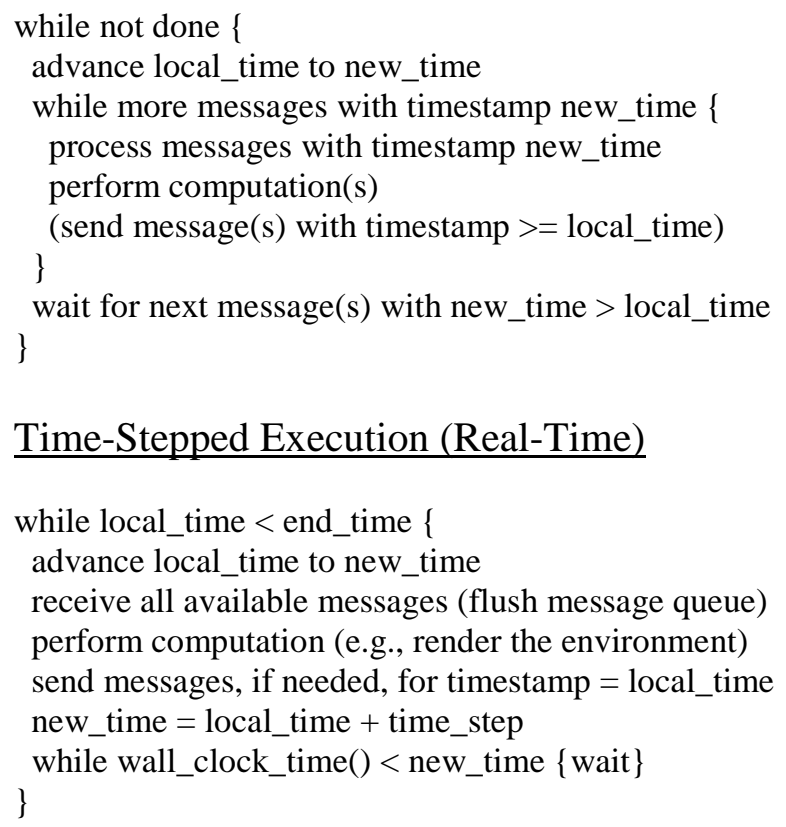

Time-Stepped Execution (Real-Time)

not synchronize their event processing at all, due to the overhead required. Practically, this means that messages in real-time distributed simulation are processed as soon as possible, in the order received. We call this receive-order (RO) message processing.

\section{Simulation Execution Models}

The differences between analytic and interactive simulation paradigms can be seen in the models of execution of simulation processes. Table 3-1 shows simplified examples of processing loops for analytic (time-managed) and real-time, interactive (time-stepped) simulations. Two fundamental differences are apparent in the example process models: (1) incoming message order and (2) wait condition. In time-managed execution, the messages are delivered in timestamp order (TSO). In time-stepped execution, incoming messages need not be delivered in any particular order. It follows that, in time-managed execution, a process must wait until the correct order of incoming messages can be determined. A real-time, time-stepped execution, on the other hand, simply waits until the wall-clock time has advanced to the next time step (which we commonly refer to as $\Delta \mathrm{t}$ ).

The real-time, time-stepped execution model shown suffers from two problems that are avoided in time-managed simulations. Since the sender and receiver of a message proceed independently, messages can arrive arbitrarily long after they are sent. Some protocols, therefore, include mechanisms to compensate for latency in certain types of messages. Dead-reckoning, for example, can be used to extrapolate the state of an object from a late message. 
A more significant problem in the real-time, time-stepped execution model stems from the lack of a delivery order. During an execution, messages received independently from two logical processes (LPs and agents, for example) may convey information that is causally related. Simply explained, a message A, sent by process 0 , may have caused some message $\mathrm{B}$, sent by process 1 . Without a delivery ordering mechanism, it is possible for processes (other than 0 and 1) to receive messages in either order $\mathrm{AB}$ or BA. The order represented by BA is called a causal-anomaly and is a significant concern in the analysis of distributed simulation systems.

The parallels between real-time, distributed simulation and the ACSES architecture are important. In our system, we can treat the sources of external data as a sort of independent, real-time subsystem. The order of events in the real-world is fixed; however, the order that they are received and processed by our agent-based simulation must be regulated by our architecture.

\section{Sources of External Data}

Data for insertion into the simulation are available from several interesting sources, e.g., online sources like RSS feeds, news wire services, and even Google alerts. Generally, we hoped to use these sources to inform us of new developments in the modeled area of interest. Though the methods developed here are novel, connecting real-world data to a running simulation system is not a new idea. In the defense-related distributed simulation world, connecting simulations to test ranges can provide additional complexity and realism to those environments. The Test and Training Enabling Architecture (TENA) provides standard methods for integrating many types of real-world hardware, range equipment, and simulations. Figure 3-1 shows the logical structure of a TENA system. 
Figure 3-1. Logical structure of a TENA system.

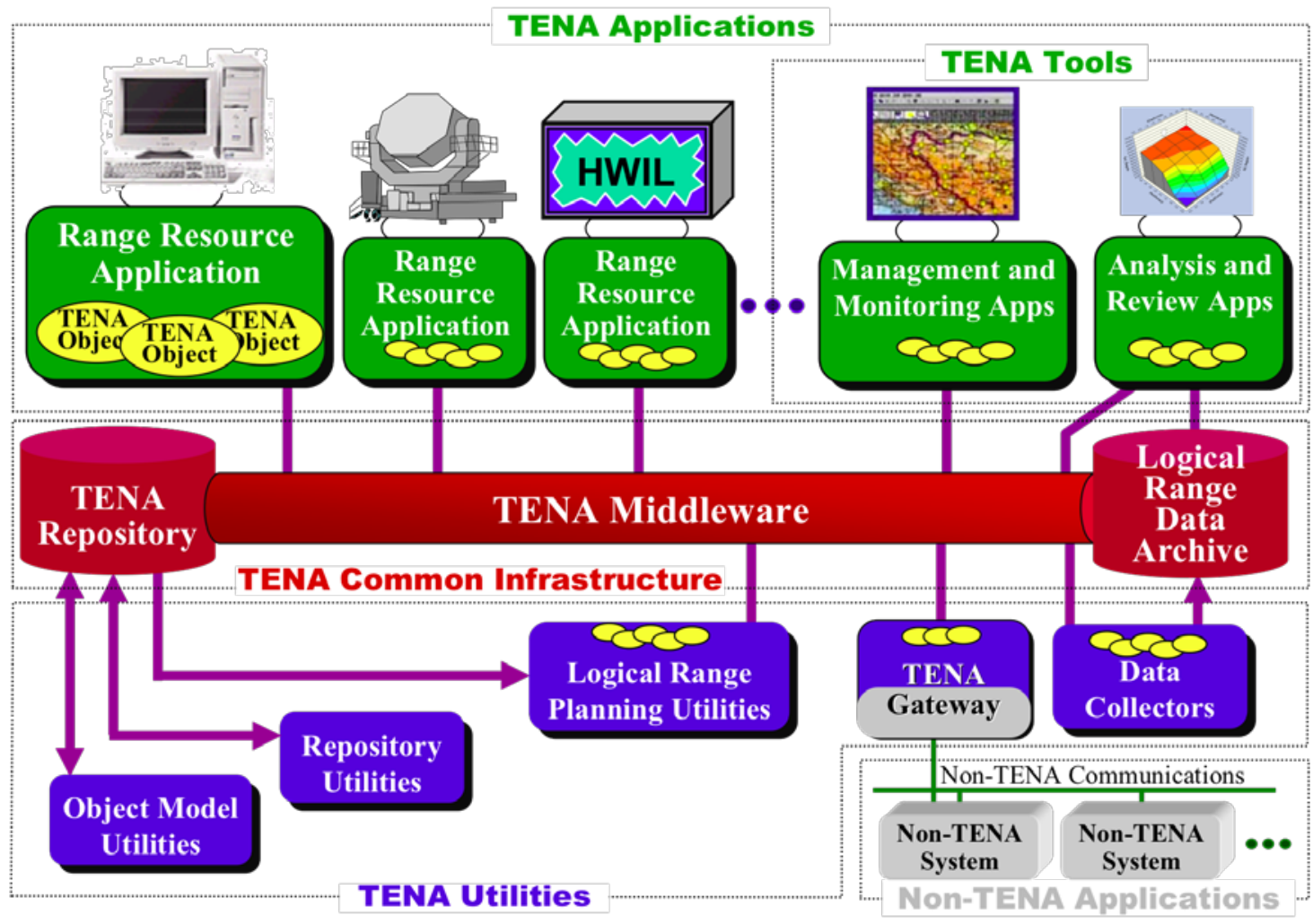

In a paper on the use of data-driven simulation for social sciences, Kennedy and Theodoropoulos suggested architecture for using agent-based simulations connected to real-world sensors (Kennedy and Theodoropoulos 2005). This is shown below. They went on to suggest that the models of the world within the simulation could be driven by these sensor agents; however, they did not specify a methodology. The system they proposed includes a feedback loop, of sorts, in that they also suggest the possibility of "effectors" that could alter the state of the real-world based upon some chosen course of action. 
Figure 3-2. Data-driven agents

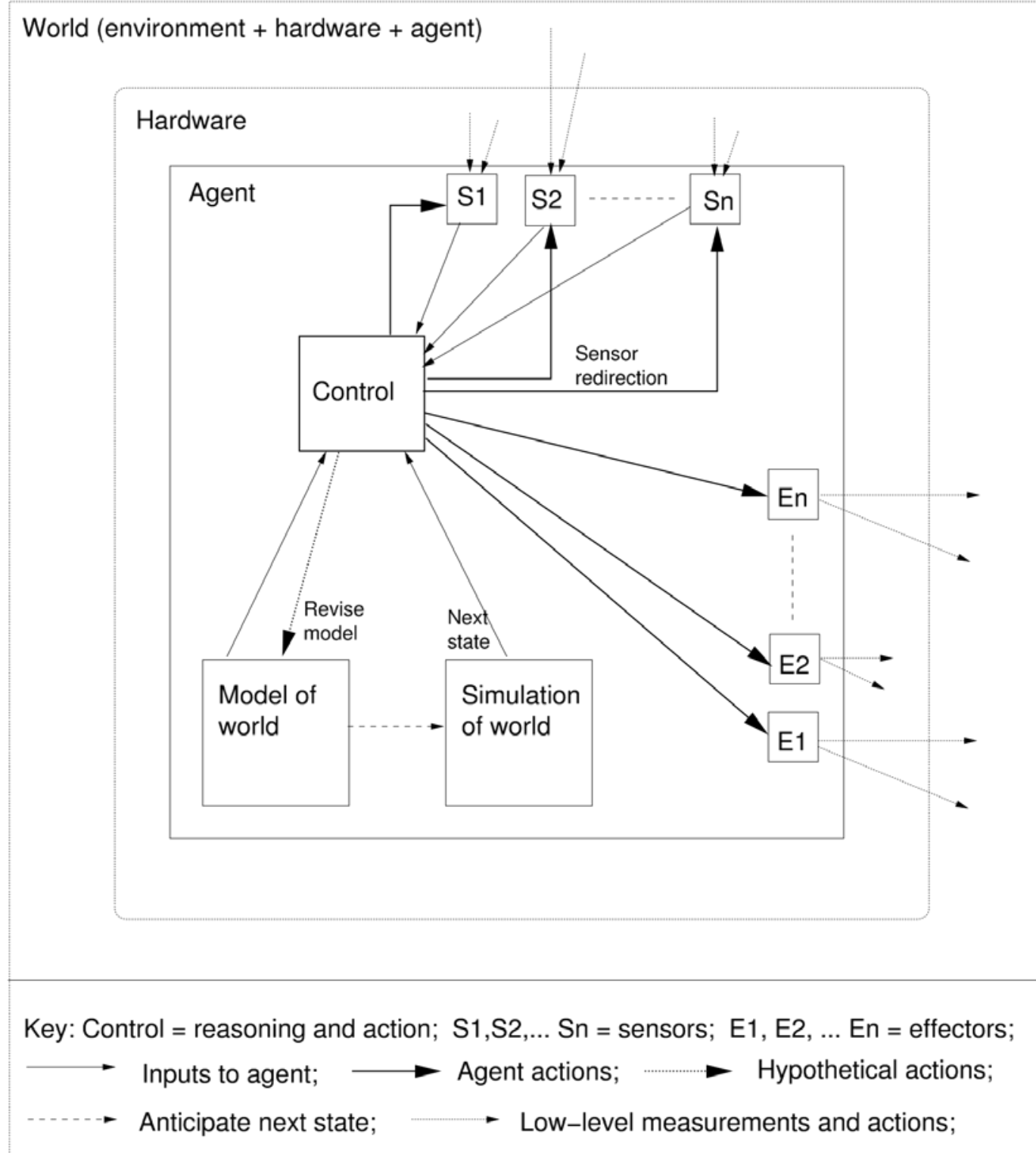

Weather simulations provide another parallel to the ACSES program goals. In the LEAD project, Plale and others (2005) describe a process for updating forecasts based on simulations where the current conditions are constantly changing. 
Figure 3-3. A process for updating forecasts (Plale 2005)

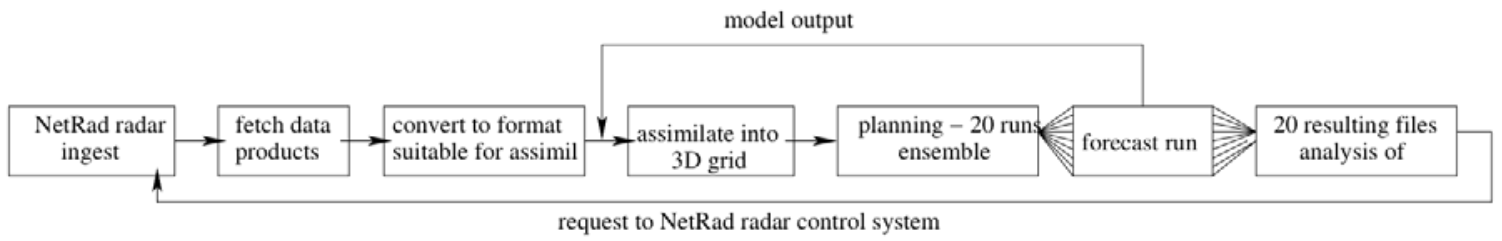

Figure 3-3 shows their process flow. Currently, computer models for forecasting weather must be rerun with new data at each observation. This process takes time and does not capitalize on the previous computation.

Figure 3-4. Comparing models for updating forecasts

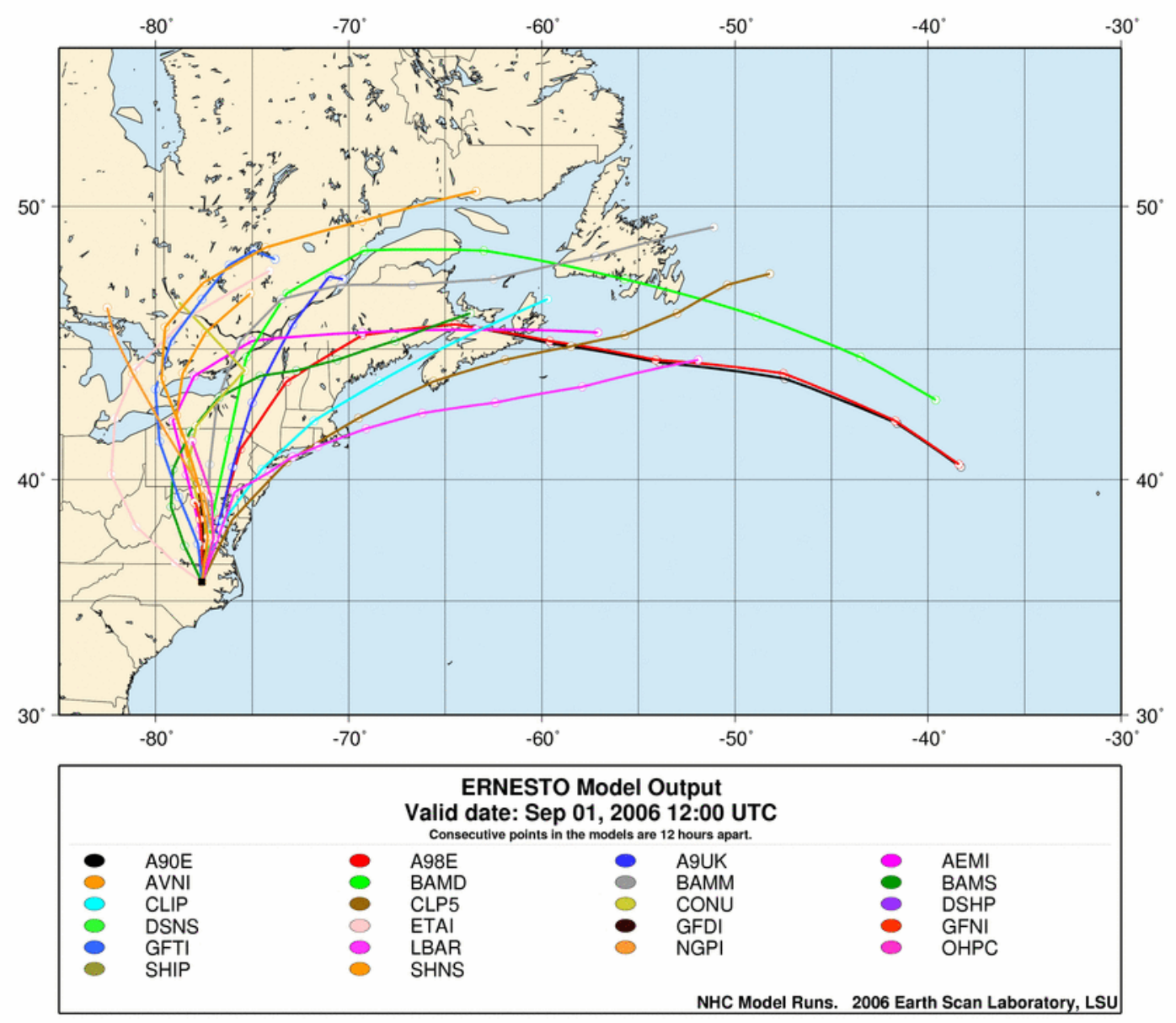

(Source http://www.esl.lsu.edu/quicklinks/hurricanes/2006/ERNESTO/) 
Moreover, a set of computer models may produce widely varying predictions, as is illustrated by the numerous models shown in Figure 3-4. The figure shows the independent predictions of a set of models using the same initial conditions. Even though some of these predictions ended up being more accurate than others, no effective way existed to incorporate the observed accuracy of a particular model into subsequent runs. This problem parallels our issues with ACSES predictions.

\section{Autocoding of Free Text and Real-Time Event Generation}

Our presumption, in the study of insertion of real-time events, was that the best sources for relevant data would be online sources. We assumed that news feeds, Google alerts, and media websites would provide near real-time information that was otherwise unavailable. We also understood that other, nonpublic sources might be available, such as remote sensors, command-and-control systems, and similar military-type inputs.

Although the format and meaning of non-public sources is fairly well understood (typically using prescribed protocols for information exchange), the content of public sources is much more difficult to deal with. For this reason, we examined the state of the art in free text processing.

So-called automatic coding methods are designed to take textual descriptions of events and create equivalent event data, constrained to a limited, precise vocabulary. Much of this work is familiar to the social science community, as it has been used to analyze large sets of historically significant events. We examined the Kansas Event Data System (KEDS) and its successor, Textual Analysis By Augmented Replacement Instructions (TABARI), both of which use an extended form of World Event Interaction Survey (WEIS) encoding. The Integrated Data for Event Analysis (IDEA) typology was also examined.

The purpose of autocoding is primarily to replace the labor-intensive manual coding of data required for analysis. Effective autocoding, therefore, is judged with respect to the time, effort and accuracy of a similar job by a human. A good autocoding effort can be applied to vast amounts of event data efficiently. The effort associated with autocoding is nicely typified online by the KEDS team (excerpt from

http://web.ku.edu/keds/index.html):

\section{STEP 1: Locate and reformat a set of machine-readable texts}

\section{STEP 2: Develop the initial coding dictionaries}

\section{STEP 3: Fine-tune the dictionaries}

\section{STEP 4: Autocode the entire data set}

\section{STEP 5: Aggregate the data for statistical analysis}

The suggested steps in the KEDS process are significant to us in three important ways. First, they presume the ability to reformat machine-readable texts. For our systems, many such sources of text exist. We would like to be able to concurrently exploit many sources 
of information, but the methods for reformatting the various texts are not the same. For example, the structure of a data feed from AP wire may not be the same as from CNN. We would require an additional step to reconcile the various formats. Moreover, some of the structure or style of the different sources may produce different coding for the same event.

Second, the autocoding process expects that dictionaries can be derived for all the significant events in the system. This may be a reasonable assertion for a limited, retrospective analysis. For a running, continuous update, the dictionary would also need to be modified. For example, simple changes to terminology, like "suicide-bomber" being altered for a while to a less-precise "homicide-bomber," can create ambiguity in the parsing of text. Practically, this means that a human would have to scan each incoming text to ensure that the dictionary accounted for the terms used and that the definitions were consistent.

Third and perhaps most importantly, step 4 and 5 imply that the proper way to analyze and understand the success of the autocoding is in a cumulative, statistical analysis. This makes sense, because the success of autocoding is measured in aggregate and does not depend on successful encoding of one or two very critical events. In our scenarios, the correct encoding of certain critical, possibly unexpected events is key to our success.

\section{Anticipated Benefit from Runtime Input}

Keeping the execution of the simulation bound to real-world data has clear analytic advantages. As in the examples for weather prediction, we can use the data to ensure our simulation does not become obsolete. Automating the process of incorporating new data would allow the simulation to automatically re-run any execution results that were invalidated by new events. This facility has several other possible uses.

- What-if analysis - By implementing a general-purpose external data input we need not be tied to specific devices or sources of real-world information. We can, if we choose, create fictitious data to perform whatif analysis of the modeled system. By creating future events and inserting them into the running simulation, we can, for example, assess the impact of an assassination attempt, or the outcome of an election. We can also explore the impact of reordering events, such as withdrawal from different parts of a region.

- Validation - With a sufficiently robust simulation, we should be able to use certain observations about the population to verify that the simulation is progressing correctly. By measuring and comparing some attribute of the modeled population and the simulation population, we should be able to determine a metric of convergence or divergence between the two. It should be noted, however, that agent-based simulations of social systems can often exhibit dynamical properties that are not easily compared. [See Gintis (2007) for a detailed explanation.]

- Goal states for adaptive control - Another use of external data is as a goal state for a learning algorithm. By allowing the simulation to explore different states and transitions, the system may be able to discover one or 
more configurations that produce the observed event. Similar to hidden Markov modeling, this allows the system to reproduce the behavior seen in the real-world, while not being prejudiced toward one specific behavioral mode. The approach may help validate possible assertions concerning the governing dynamics of the real-world population.

- Surrogates for difficult models - Some objects or activities in the modeled system may be difficult to construct. Simulation of rare events and of complex interdependent behaviors is challenging and can be computationally intractable. Further, a system may have certain key actors, like leaders or government officials, whose actions cannot be predicted by a simulation, but whose impact is critical. In such circumstances, an external input can serve as a sort of script for that individual's behavior. By relating external events to that actor's agent, the agent can generate behaviors appropriately interleaved with other agents. The events can simply be played back, or can be used to trigger a state that will produce a different response to other agents. External events can be an effective way to augment agents with complex behavior, without computational costs.

\section{Problem Description and Conceptual Model}

We began the task with an understanding of what would be required to insert events into an executing agent-based simulation. The two basic issues are synchronization and mapping. The synchronization problem involves regulating the simulation execution such that the event can be inserted at the right point in the computation. The mapping problem deals with determining how an event, with explicit content and meaning in the realworld, should manifest itself as a change to the state of the simulation. In this section we describe these problems more explicitly for our context and provide an abstraction for addressing them.

\section{The Synchronization Problem}

All discrete event simulations have a synchronization model, used to order the processing of events. In simple, monolithic simulations, this may simply be a queue of events. In more complex simulations, the progress of the simulation is related to an internal clock or counter. The value of the clock is referred to as simulation time. To insert an external event, we have to create a relationship between simulation time and real-world time and create a device for processing the event at its indicated time-stamp. In NetLogo, the only notion of time is the "tick" or time step. The progress of a simulation is measured in these ticks. Any number of logical operations can happen between ticks, and the advancement of ticks can be controlled manually. The tick counter can also be reset to zero. It seems natural to exploit the tick function in addressing the synchronization problem in NetLogo.

Maintaining relationships between independent clocks is explored in detail in Lamport (1978). In this situation, we need to do two things: (1) create an equivalence between a tick value in the NetLogo simulation and a real-world time and (2) create a method for 
relating the advancement of ticks to real-world time. The first part creates an "anchor time" in the real-world that can relate to a specific tick value (initially zero, of course). Given this anchor, the second part is simply a way of determining one clock value from the other. For equal time steps, we can use a linear function to express this relationship:

wall-clock $=$ anchor-time + tick $*$ timestep

With a constant time step, this relationship can be used throughout the simulation. Unfortunately, it is frequently necessary to change the size of a time step during a simulation. Simply modifying the value of time step in the above equation will not work, because calculations have already been performed at the previous time step and cannot be scaled. Without additional changes, simply allowing the value of time step to change would have the effect of an instantaneous change in time, forward or backward. To solve this, one can reset the anchor time to the current wall-clock time.

We created an extension that maps the tick to clock time by specifying a scale factor. Further it provides a call that retrieves events relevant to the current time step for processing.

Figure 3-5. Two-step transformation process

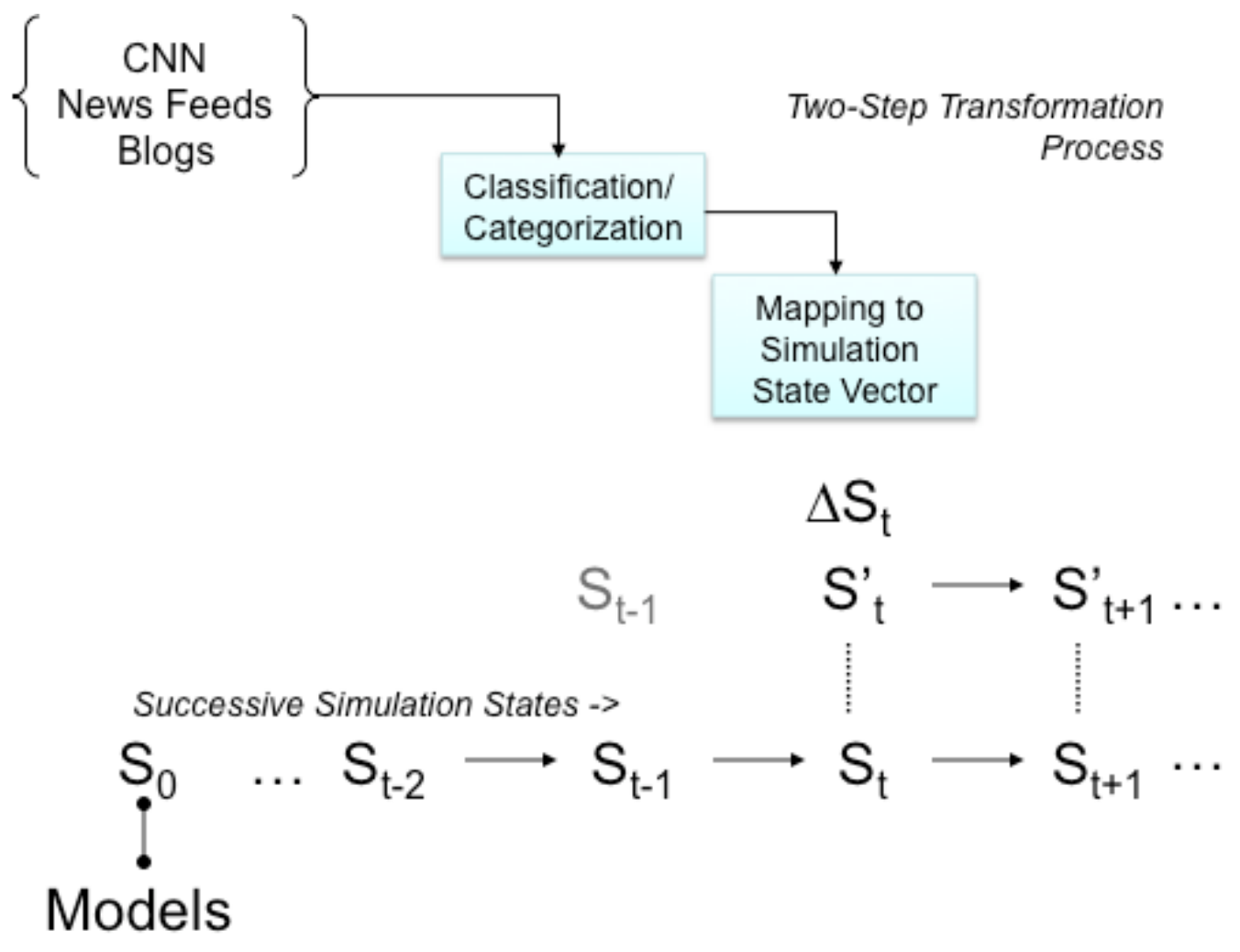




\section{The Mapping Problem}

Fundamentally, the need to insert external events implies that the events were not modeled within the simulation, or that they did not occur in the simulation execution. The challenge, therefore, is to determine what effect those external events would have had on the simulation state. In a sense, the event insertion mechanism is a surrogate for models of phenomena. The problem become relating the external description of the event to a change in the simulation state. This is depicted in Figure 3-5.

The mapping problem is related to the study of linguistics, ontologies and semiotics. Mapping from real-world semantics to simulation semantics is a form of universal translation. General solutions to the mapping problem are broadly understood to be impractical. Overly restrictive approaches will not provide much utility either, because they would need to be implemented for each type and source of data. Our approach, therefore, attempts to allow arbitrary external data and semantics, while easing the burden of custom code creation.

In determining an approach, the first observation we make is that it is intractable to try to assess all possible state changes from all possible events (or apprehensions of events). So, we assume that all of the events that can possibly occur can be categorized into sets of events for which the impact to the population will be about the same for any member. This forms an important first step: mapping free or unconstrained text to categorical data. Autocoding utilities, such as KEDS and TABARI, perform this transformation. In those cases, the events are transformed into WEIS codes. The approach we pursue here is not restricted to any particular code set, and IDEA or arbitrary coding would work just as well.

\section{Channels}

The result of categorization is simply a vector of data values and a real-world timestamp. We refer to this vector as a channel. A channel delivers a specific class of event and is associated with a particular source. In practice, it will be necessary to refer to a channel by some means, so we might allow an arbitrary label. Thus we can define a channel:

channel name att-list;

For example, a channel delivering events parsed from Reuter's newsfeed parsed by TABARI would be

channel reuters weis-code subject direct-object goldstein-wgt;

\section{Event Handlers}

This structured input format is the entry point for the second step of the process, the event handler. Since the category data is not yet related to the simulation semantics, we must 
allow the user to specify how this data will change the simulation. This implies a choice of mapping logic based on the channel and event type. By defining a mapping function available to a channel together with the channel descriptor, we have an attribute signature that can be used to select the appropriate function. In essence, we can use the values of the event attributes to determine the appropriate mapping logic.

The handlers themselves perform the heavy-lifting of the mapping process. They must deal with both the categorical data and the simulation data. We implemented a way of calling arbitrary simulation code upon the processing of an event. This allowed the person incorporating the external data source to express the needed changes in the semantics of both the external data and the simulation models. The method for doing this is an event handler, which specifies both the source data to be used and the transformation processes for the events.

event event-label $(*<$ attribute_matching_logic $>)\{\langle$ java_code $>\}$

For example, the code below specifies that an event with any value for the first attribute (location) and with a value of 10 for the second attribute (amount) will highlight the location of that event.

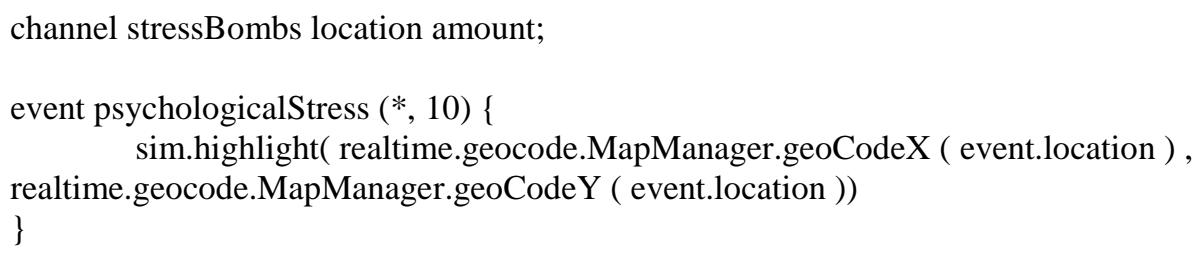

\section{Transforms}

In defining the mapping process, it was apparent that some transformations would appear frequently. Notice the geocode example above. To accommodate arbitrary transforms without the need to recompile NetLogo, we added the notion of a transformation as part of the mapping logic. A transform is a function that performs a mapping between a symbol in an event to a construct in the semantics of the simulation itself (in NetLogo, a set of agents or patches). For example, Address() would be a transform that mapped "Golden Gate Bridge" to (x,y) in simulation space, while Near() would transform "Golden Gate Bridge" into a set of agents. These are intended to be primitives, and therefore transforms are simply signatures that map via native calls to the running simulation.

transform name event-attribute list to simulation-type with native-call

A transform that would be useful for the above example might be

transform Address subject direct-object to location with realTime.googleAddressLookup;

Simulation-type can be one of the following: 
- agent

- agents

- location

- locations

Transforms can return a null value if the particular set of symbols does not map to an event. By default, a transform is assumed to map to all possible values of an attribute it is concerned with. For example, Address maps to "Golden Gate Bridge" but could also map to "Joe Random." An event that handles "collapsed" for a bridge probably will not have the desired behavior for the event "Joe Random collapsed at work today."

transform Address subject, direct-object in [“San Fransisco”, “Golden Gate Bridge”, "Bay Area”] to location with realTime.staticPlaceMap;

\section{Implementation and Examples}

Our implementation allows NetLogo models to accept synchronized, external inputs from a variety of sources. We have included extensions to NetLogo that implement time keeping, facilitate geocoding of locations to latitude-longitude (lat-long) coordinates, and prescribe external data sources and transformations for event data. Figure 3-6 shows the components of our extensions. The timer or wall-clock extension allows a user to specify a relationship between ticks and the cpu clock. Geocode uses the Google maps API to do easy transformation of location names to lat-long coordinates. It communicates directly with google.com and therefore requires internet connectivity for geocoding.

Figure 3-6. Notional geocoding construct

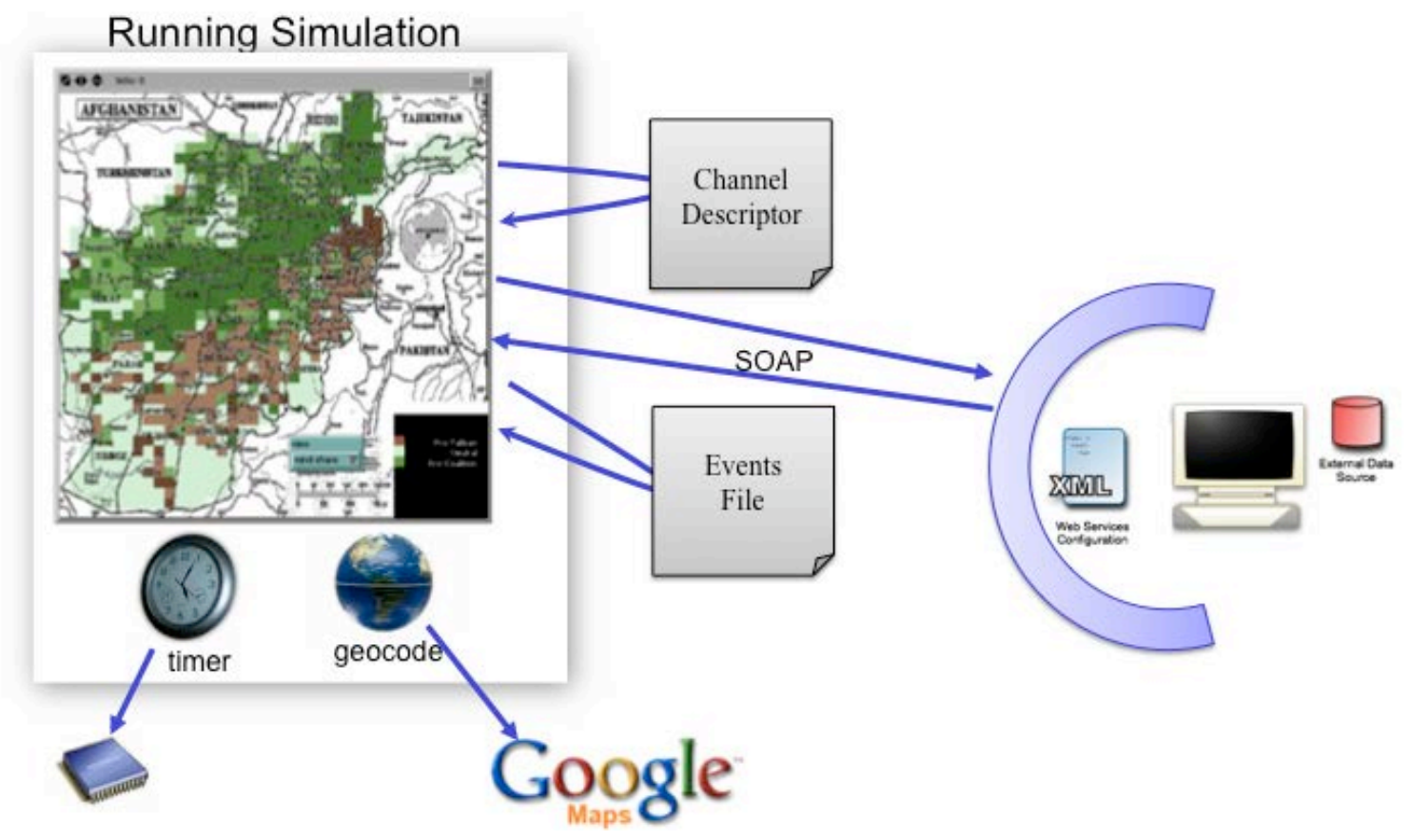


Mapping data and inserting the events are performed by the simframe extension. This extension allows users to register channels with the simulation, as sources of external data. These sources may be simple files, or they may be URLs, indicating a remote data source. Simple Object Access Protocol (SOAP) requests, a web standard, are used to get events from the external data source. In both cases the event data must be preformatted and include a timestamp. The format expected by simframe is compatible with the output of TABARI. An example of an event file is shown below.

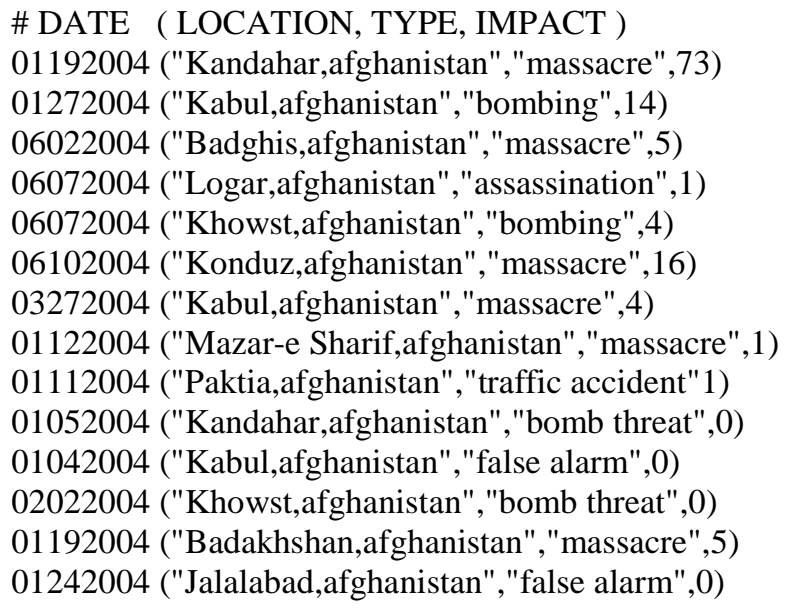

Example event file

An example SOAP event server is also provided to illustrate how easy it can be to set up an external data source for simframe. In the example, the SOAP server simply reads a file and provides the events, but it should be clear that the file data could be replaced with any data available on that server. Thus, instrumenting a sensor or remote website to provide data feeds is a straightforward job.

The channel descriptor file, in addition to containing the source information, also includes the logic for selecting a transformation and performing the update to the simulation.

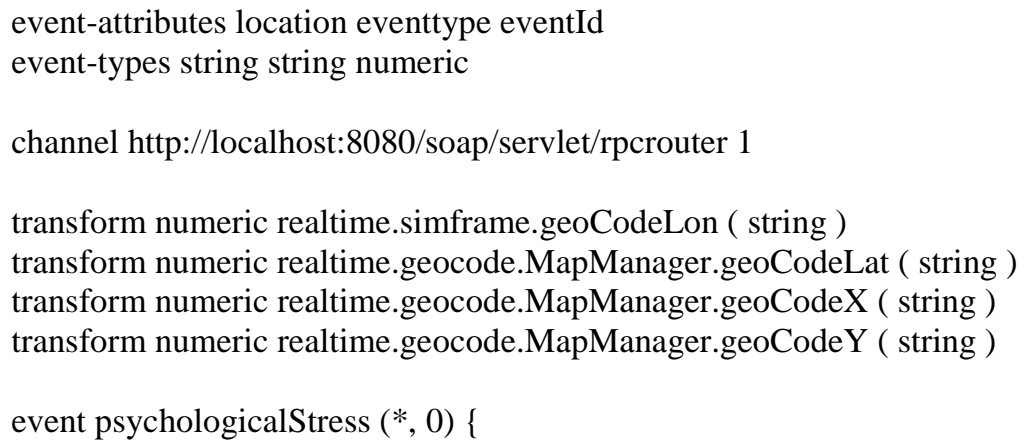




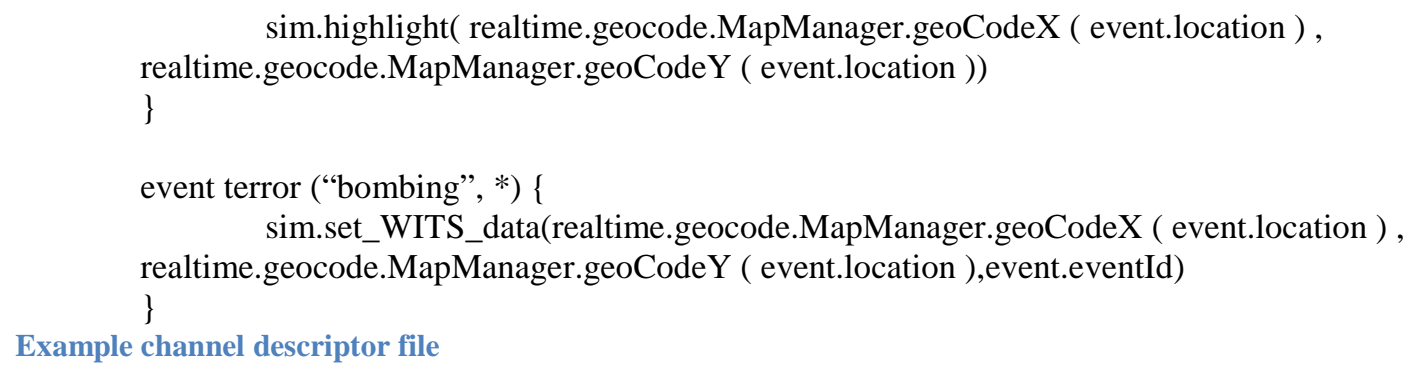

Descriptions of the example NetLogo simulations are provided in Appendix 7. Each simulation includes an explanation of the function of the extension it demonstrates, as well as instructions on how to use it in a more complex simulation.

\section{Autocoding and External Data Sources}

Our insights regarding autocoding are that the technology requires a robust and temporally expressive ontology of events and the ability to characterize and extract the relevant information in computationally tractable times. Moreover, problems of reconciling and collating events and capturing multiple perspectives of a single event is difficult.

To handle the volume of documents that could explain relevant events, documents and cable documents and knowledge discovery tools are rapidly evolving. Currently employed systems include the PACOM deployed ORNL-developed Piranha system, although other systems are also in use.

Piranha was first installed at PACOM’s Virtual Information Center in 1997. More recently, ORNL has worked with PACOM to improve the performance of Piranha by utilizing parallel processing in 2005- 2006 to address the document volume issue illustrated within this chapter. Piranha was subsequently installed at multiple intelligence center (IC) locations. Piranha has been developed and trained to search and retrieve realtime information from sources such as news agencies and the Internet. It looks at thousands to millions of documents and in a matter of minutes selects the 50 to 100 most relevant documents for in-depth analysis.

The real-time streams of data to the ACSES system may be of similar size, and relevant events must be quickly selected for insertion into the simulation. Piranha can be deployed to retrieve real-time information, de-duplicate, and screen data, and an informationextraction software component can be inserted to extract events from the data produced by Piranha. These events can be incorporated into the ACSES simulation as real-time updates. If analysts are to manually capture these events, this knowledge discovery approach is crucial to making real-time updating feasible.

Any negative insights about autocoding are more than offset by our discovery of the wealth of this type of coded data already available. Several government and nongovernment agencies provide large data sets of world events, frequently geocoded to the region or city. A few online sources can be tapped in real-time. Notable among these is http://www.globalincidentmap.com, a private endeavor that continuously monitors and reports on various types of events. 


\section{Mechanics of Event Insertion}

The intermittent nature of important events necessitates a certain execution model. This is commonly known as optimistic execution. The utility of the online analysis is driven by the ability to predict. This means executing into the future. The arrival of a new event (in the present) will require the simulation to be rerun from the point of the new event forward. If we require the exploration of many alternative futures (an important feature, of course), then the simulation system will need to manage many states, separate threads of execution, and outcomes. The management of this type of predictive simulation capability is beyond the scope of current analytic simulation engines.

\section{Specifying Affected Agents}

We have realized that the agents affected by an event can be specified in two important ways: (1) by a geographic region and (2) by communication-connection pattern. For geographic impact, the simple solution is to place an effect at a position and let the agents sense them based upon their distance. For more sophisticated propagation, we must determine a way to select agents on the basis of their connections to media or to each other, then meter the impact taking into consideration the characteristics of the connection network.

\section{Future Work}

The primary remaining work is to investigate and tune the algorithms for affecting the agents for each type of event. Our current implementation simply "irritates" all agents in the specified region. A better model will discriminate on the basis of agent alignment, the cause of the event, and its nature. We are hopeful that events like those provided by the Worldwide Incidents Tracking System (WITS) or the GlobalIncidentMap can be used to show realistic changes to the motivation and subsequent behavior of a population.

We also hope to explore more sophisticated analytical techniques suggested by the need to insert events and rerun simulations. Because this will produce a large space of possible futures, it is important to be able to efficiently process and analyze the data, gleaning insights quickly as the situations change.

\section{Insights}

In this chapter, we detailed the design and implementation of a method for insertion of external data into a running simulation. Our contribution to the state of the art is most significant in our generation of code to support arbitrary online sources of data. We have implemented a web-services-based method for creating and connecting to any data source that can be converted to appropriate categorized events. This means that we can potentially tap into any available online feed, through either source or local conversion. Additionally, of course, we've augmented the NetLogo toolset and proved the viability of our approach.

The most interesting problem presented by the work to date is the efficient simulation of vast alternative spaces, updated in real-time. To efficiently manage this type of 
computation, one needs to address resource management of memory, processing power, disk space, network bandwidth, and more. To do this effectively, the simulation must also be analyzed in real-time to provide clues concerning the relative importance or redundancy of a particular vignette. This places new and interesting requirements upon high-performance computing applications.

To handle the volume of documents that could explain relevant events, documents and cables and knowledge discovery tools are crucial to allow feasible real time updating. Currently employed systems can be deployed to retrieve real-time information, deduplicate, and screen data, and an information-extraction software component can be inserted to extract events from the data produced by Piranha. These events can be incorporated into the ACSES simulation as real-time updates.

Other challenges suggested by this work include the analysis of temporally indeterminate Kripke structures (a way of expressing a computation as a state diagram, modified to include equivalence relationships, and critical attributes for disambiguation of states) and exploring the viability of generating complex adaptive system (CAS) covering spaces (for example, to determine how many runs of a simulation are needed to have confidence that the possibilities are understood). 


\section{Chapter 4: Accounting for Surprise}

\section{Introduction}

The research plan for the ACSES project is provided in Appendix 1 - Research Plan. A detailed description of our methodology for training the agents is provided in Chapter 2. Briefly, we evaluated (1) real-world data sources, their quality and availability (Chapter 1); (2) a distributed GA for training the simulation (calibrating) based on a subset of this data in static form (Chapter 2); and (3) the feasibility and technical challenges for utilizing real-time (nonstatic) data in the simulation (Chapter 3). For a detailed description of the NetLogo CAS simulation, please refer to Chapter 2: The NetLogo Simulation.

This chapter will document our efforts to identify and explain surprise in the CAS simulation of Afghanistan. We also explore causal factors for these behaviors through a partial-order temporal analysis of the state space of our model. Appendix 8 reviews methods and issues surrounding the construction of plain-text narratives from event-level simulation data. Appendix 9 details the extended toolset we have created for more direct and intuitive manipulation and analysis of the NetLogo model.

\section{Surprise: An Overview}

The purpose of a CAS simulation is necessarily two-fold: to explain the causal reasons for known significant events and to predict unknown significant events. In the first case, we must know the environment and the events in order to hypothesize the causes; in the second case, we create a likely or interesting environment, and then we use the hypothesized causes to see what will happen.

Many of the underlying causes in a CAS are things that, by their nature, cannot be readily measured. So we create agents that have certain properties - both attributes and rulesand then we calibrate the attribute values and the rules so that the outputs of the system match the macro-level events that we can measure. These refined attribute values and rules become our hypothesis to be used in the prediction stage.

The surprise is also twofold: if we are successful, we have deduced the underlying causes of the events, which we did not know previously; furthermore, given these underlying causes, we can find out what will happen in a different environment, either under new conditions or during simulated courses of action. The key element in "surprise" is that we have measurable, macro-level events that are not explicitly programmed, but rather occur implicitly. These events are therefore defined by the continuous interaction of all the attributes and values at the agent level. It is this agent-level interaction that produces the emergent, macro-level behavior we term "surprise."

In our model of Afghanistan, we have created attribute values for each agent that define such things as support for leaders, economic incentives, influence from peers, and 
reactions to violence. All of these factors, and others, combine together in a utility function that each agent runs for itself, thereby choosing which behavior seems to be best suited for that agent, under the terms we have defined. The choices are individual ones, but they also provide feedback for other agents, and for the environment. This feedback then becomes another factor in the next round of agent decisions.

In the following sections we link this description of surprise to specific examples taken from our experiments with the Afghanistan model, as implemented in the NetLogo environment. The first section describes the macro-level agent behavior of clustering and explores some possible causal determinates for this behavior, as it is not explicitly programmed into the model. This clustering is representative of social behavior emergence; that is, it is a self-organizing phenomenon that is a consequence of the agents' individual actions and changes in state as they react to other agents and to the environment. These threshold responses are measured against different levels of external stimuli, as represented by discrete input parameters for various scenario modeling. Again, the thresholds that are evident in these results are not explicitly accounted for in the set of rules that govern the agents' behavior.

We then move beyond identifying and explaining surprising behavior. That is not to say that the results from this section do not also represent emergent behavior; they do. But here we present the prediction phase of the experimental design. As mentioned previously, once we understand the causal relationships and have satisfactorily calibrated and validated the model, we can use that understanding to discover what will happen under certain conditions. As such, in this section we describe a set of simulation runs that are aimed at evaluating specific courses of action (COAs) that would be relevant for a commander and his or her area of responsibility - in this case, the Ghazni region.

\section{Agent Clustering}

Our model of Afghanistan has two types of agents: civilians and fighters. Although the civilian agents are further differentiated by their choice of affiliation (i.e., whether they are pro-government, neutral, or pro-Taliban), they are essentially the same type of agent across all three categories. Conversely, the fighter agents have fundamentally different attribute-values, depending on which group they belong to, and they cannot move between groups. These three groups are Taliban forces, Afghan government forces (AGF), and coalition forces (CF). The Taliban forces make up the "Taliban" faction; the CF and AGF collectively make up the "soldier" faction. These fighter agents can choose at each time step whether to fight, move, stay (on the current patch), or flee. They make this action choice based on their perception of their own effectiveness relative to the local perceived effectiveness of the opposing force.

These simple rules produce effects in the system that are not explicitly accounted for, but that occur as a natural and predictable consequence of these rules. One such effect is that both the soldiers and the Taliban will begin to group together. The fighter agents are more likely to survive in large groups. Hence, larger groups will emerge in the system. No rule explicitly tells the fighter agents to group together; it just happens. 
Below are images and statistics from a single simulation run. The brown dots represent Taliban agents, the green dots are soldier agents, and the other colors represent the civilians-blue for civilians who choose to be pro-government, yellow for those who remain neutral, and red for those who are pro-Taliban. The first image [Figure 4-1(a)] is from the initial setup for the run. The civilians are placed according to demographic information on Afghanistan, while the soldiers and Taliban (1500 agents each) are allocated across Afghanistan randomly. Notice that the placement or grouping of the green or brown agents follows no discernable pattern.

Figure 4-1. Simulation results at (a) initialization, (b) 50 time steps, (c) 100 time steps, (d) 150 time steps, and (e) 200 time steps

(a)

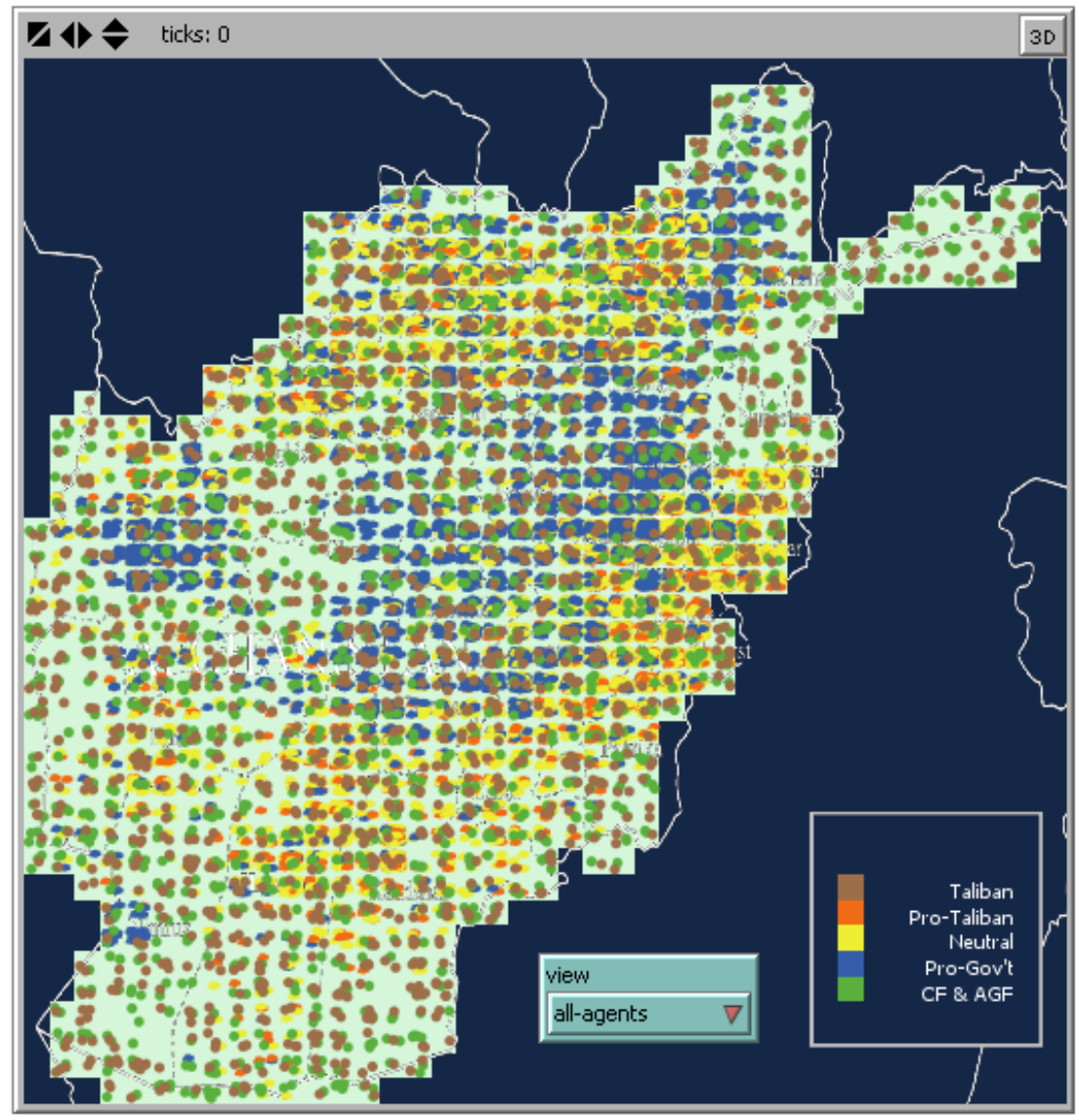


(b)

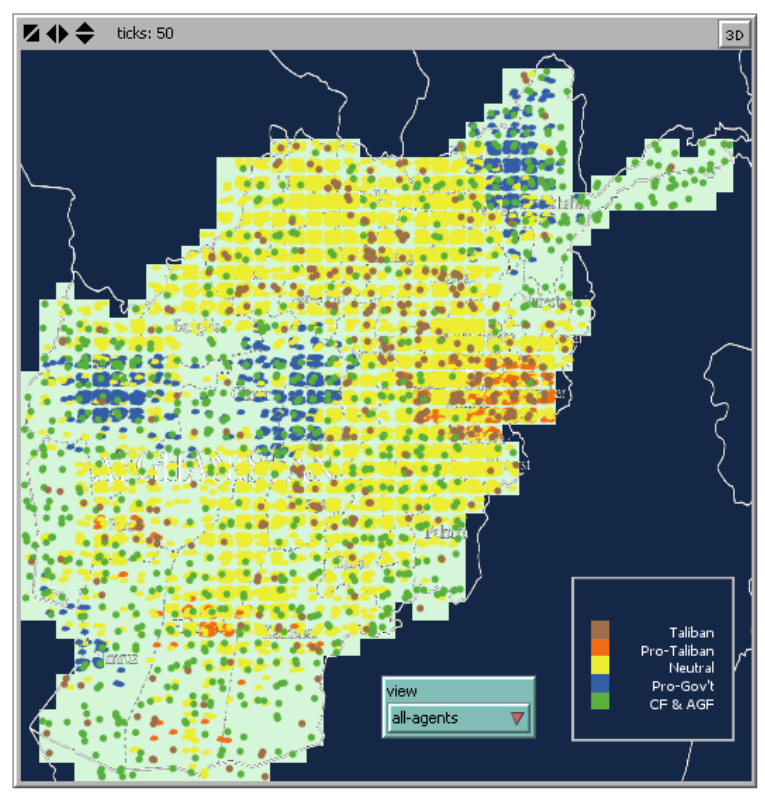

(d)

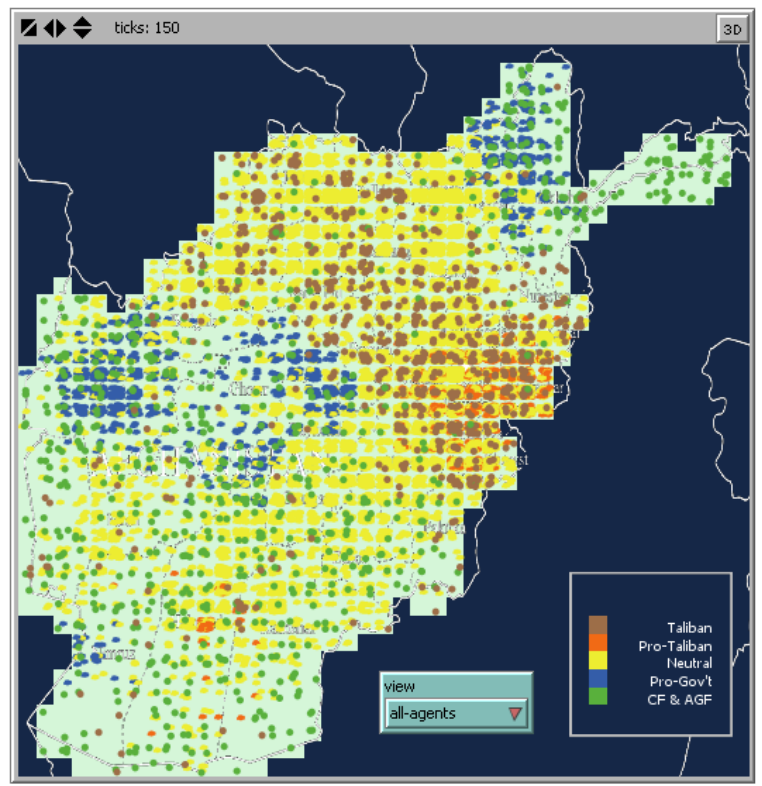

(c)

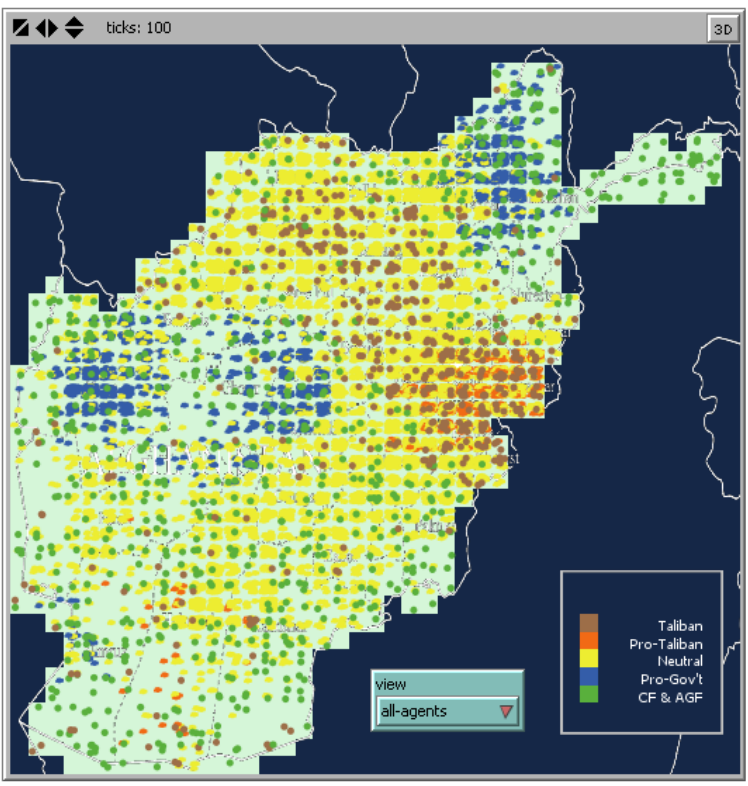

(e)

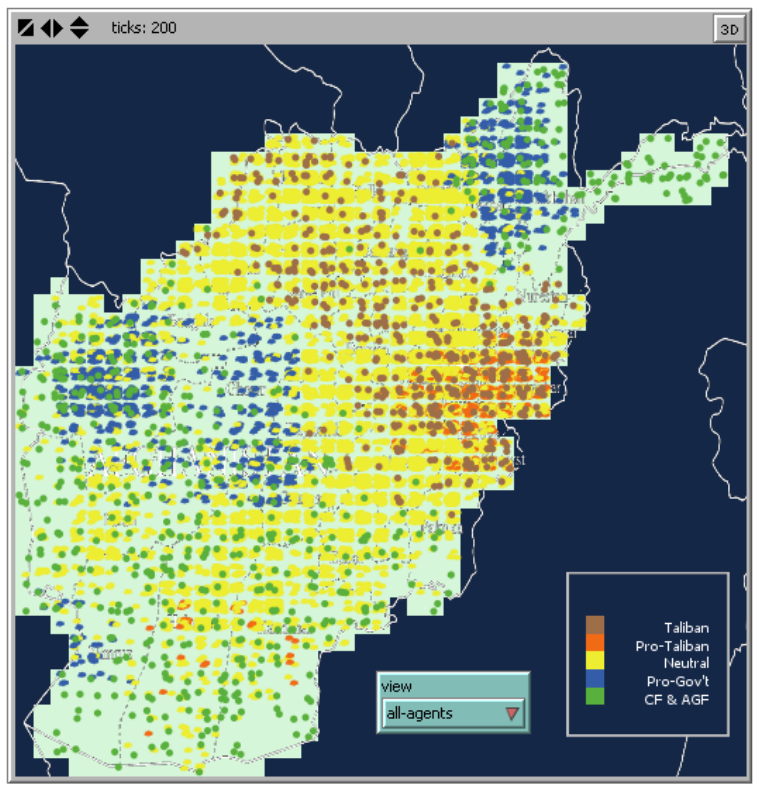

After 50 time steps in the simulation, some patterns begin to emerge. Of course, many of the Taliban and, to a lesser extent, the soldiers have disappeared from the simulation. This die-off of agents occurs in the early stages of the simulation run as, presumably, many of the weak, isolated agents have been killed. The Taliban do not have the same force strength or success rates as the CF agents. Thus, their die rate is more pronounced. 
Note that in this simulation run, 50 additional Taliban agents are injected into the system randomly at each step, while 7 CF agents and 8 AGF agents are also injected. This is done to preserve a level of stability in the number of these agents, which facilitates the study of emergent patterns during the course of the simulations.

After 100 steps, we can see a continuation of the same patterns from 50 time steps. The number of each faction has somewhat stabilized, meaning the number of new agents injected in each step approximately matches the number of agents killed in each step. At this time step, 515 Taliban agents and 778 combined soldier forces (CF and AGF agents) remain, down from an initial 1500 each.

At time steps 150 and 200, we note that areas of particular stronghold have emerged for both factions, particularly for the Taliban. At time step 150, the injection of new agents was turned off for both factions, to see the continuing effects with no new agents placed randomly across Afghanistan. In the end, 478 Taliban and 631 soldiers remain, with the majority of the latter (442) being the more powerful CF agents.

As we can see, the Taliban agents dominate mainly in the Eastern-Central area, but they also control the region that extends northwest from there, up to the northern border of Afghanistan.

During five additional independent simulation runs, the same simulation parameters were used, but the simulation was extended over a few hundred more time steps. Furthermore, the infusion of new agents was manipulated so that the number of each faction at the end of the simulations would approximately equal the initial settings, i.e., 1500 for each faction. Images of the results are shown in Figure 4-2.

Figure 4-2. Simulation results from (a) Run 1, (b) Run 2, (c) Run 3, (d) Run 4, and (e) Run 5 
(a)
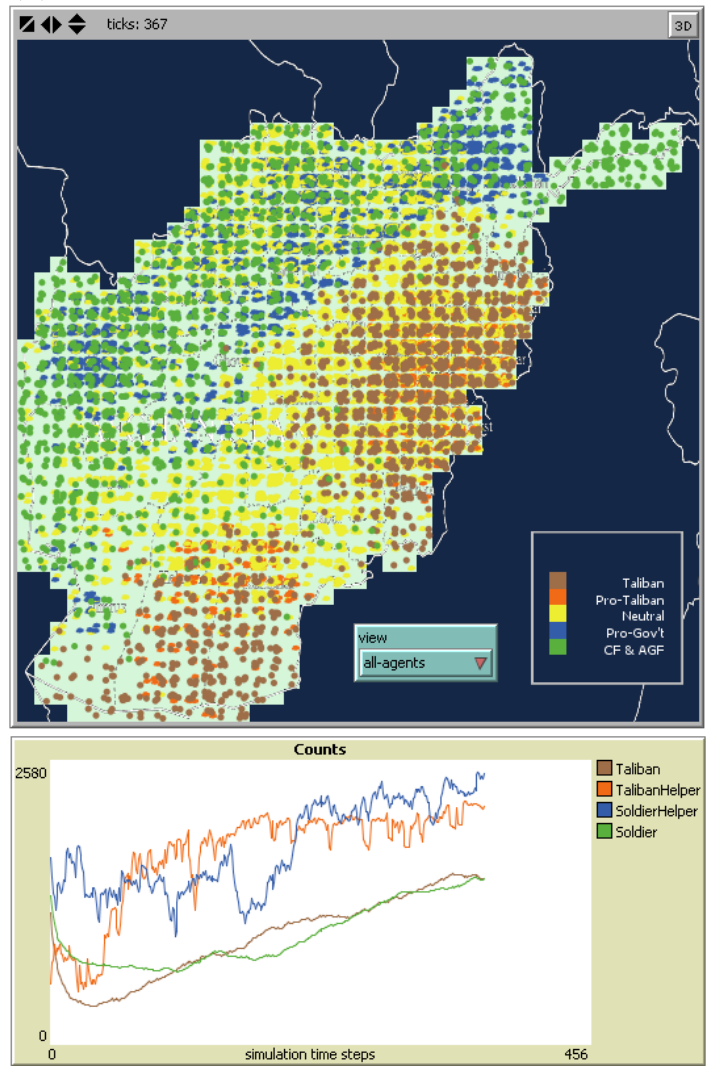

(c)
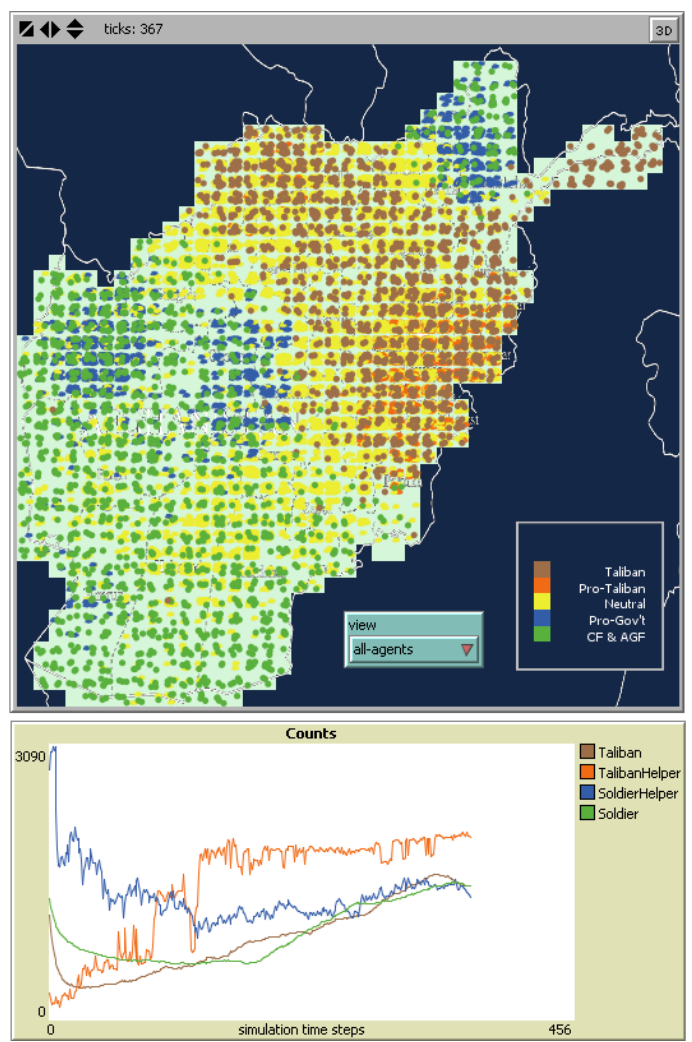

(b)
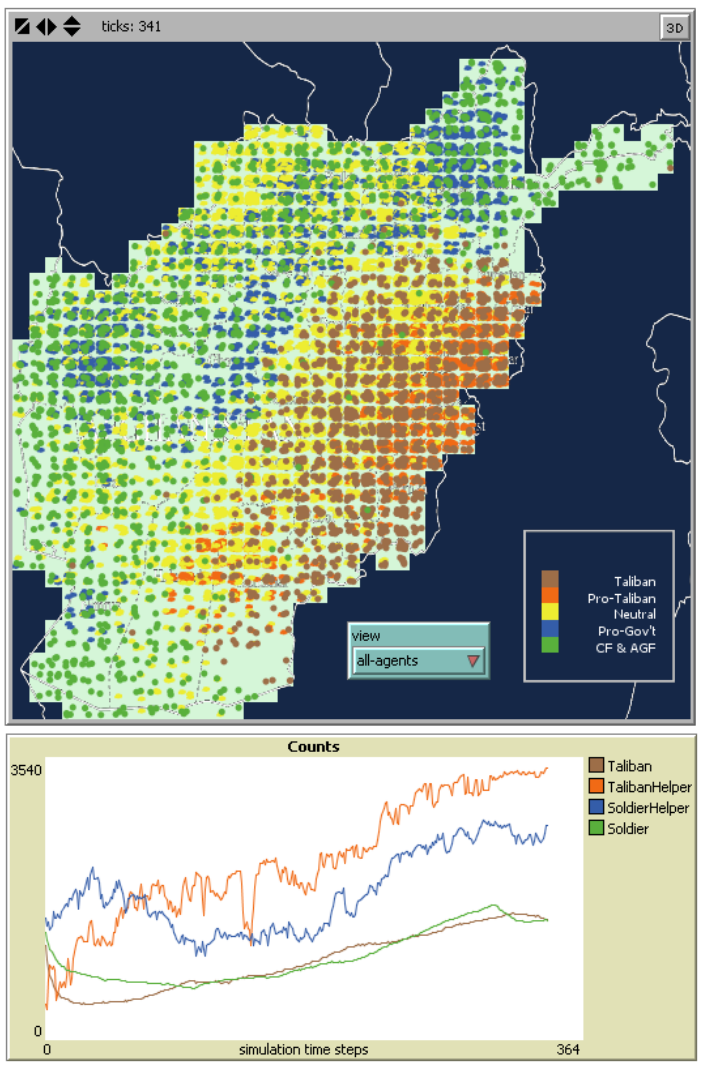

(d)

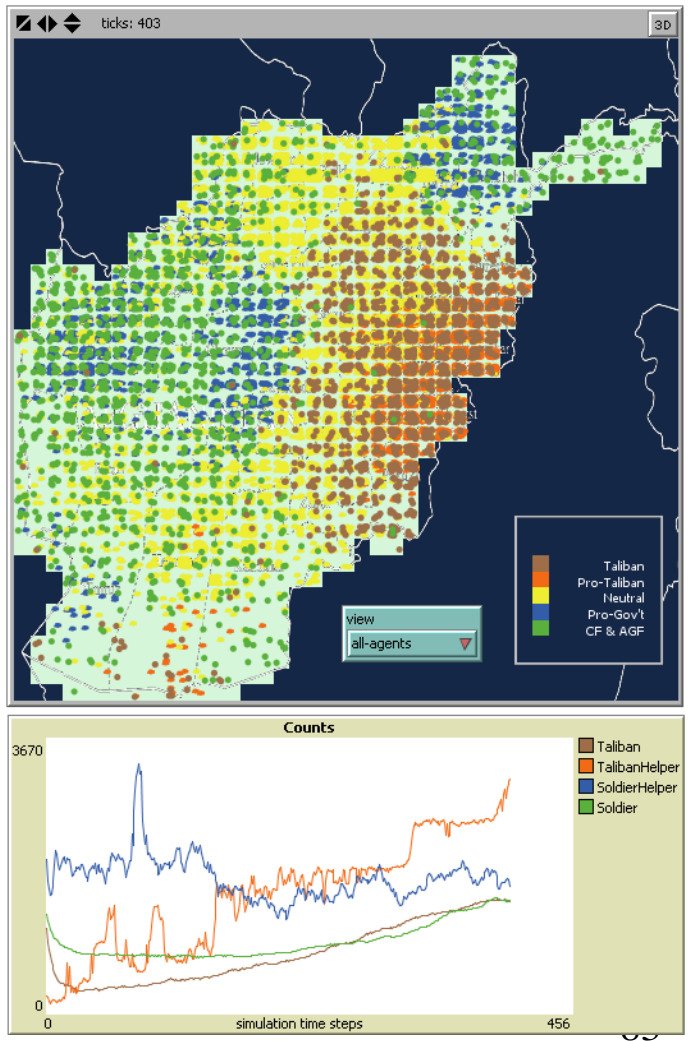


(e)

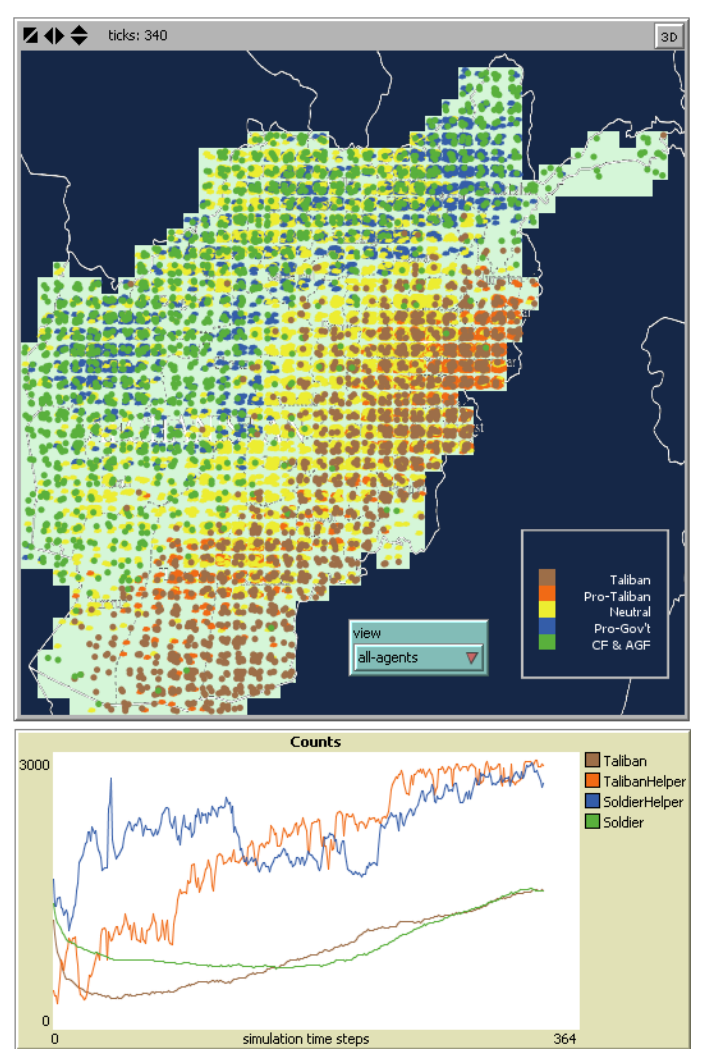

Now we can see more clearly that the Taliban seems to always dominate the EasternCentral portion of the map, but sometimes the Taliban's control extends northwest from there, and sometimes it extends southwest. In Figure 4-2(a) and, to a much lesser extent, in Figure 4-2(e), the Taliban presence seems to form two distinct groups rather than a single large group. Nevertheless, these runs support the theory that the Taliban agents will tend to cluster together to increase their chance of survival, a trait that is not explicitly programmed into the simulation.

The process of analyzing these results is like that for any similar simulation. Because many of the effects that we see are not explicitly programmed, we must explore the results and try to determine causality, which will hopefully give us insight into the real situation that we have attempted to model.

For example, we can clearly see that in all five runs above, the Eastern-Central area is always dominated by the Taliban. Two hypothesized reasons for this effect are (1) this area tends to have the highest population density and (2) this area tends to have the most Taliban support among the civilians. In terms of population, further investigation shows that a small but distinct correlation exists between population size and the number of Taliban agents (Pearson correlation $=0.227$, significant at the 0.01 level). Moreover, all 15 of the highest-density patches have some Taliban agents present (anywhere from 3 to 20 per patch) and no soldier agents. In the 60 highest-density patches, only 12 have any 
soldier presence at all, with two of those cases showing that the Taliban still outnumber the soldiers. We speculate that the difference in perception ability between the Taliban and the soldiers (particularly the soldiers who are CF) is the main influence for this trend. Because the soldiers tend to wrongly identify civilians as Taliban, they perceive a larger Taliban force than really exists in the high-density areas, simply because these areas have more civilians. Therefore, the soldiers will decline to engage whatever Taliban are there, allowing the Taliban to grow stronger in these high-population areas.

In terms of Taliban support among the population, we can clearly see [Figure 4-2(a)] that even early on, high-density areas are also areas of greatest density for Taliban helpers (in red). The role this plays is difficult to measure, however, because a feedback mechanism is at work here. Clearly, as the number of Taliban increases, the number of Taliban helpers also increases. The Taliban helpers number the lowest in Figure 4-2(a) (2157) and Figure 4-2(c) (2041). The other three runs have 3401, 3119, and 2868 Taliban helper agents [Figures 4-2 (b), (d), and (e), respectively]. Notice also that across all five simulation runs, Taliban helper agents seldom reside in places that do not have a Taliban presence.

Remember that all five of these runs have the same number of Taliban and soldiers (within +/-5), yet the relative positions of Taliban and soldiers within Afghanistan can create a range of 1500 or so among the Taliban helper agents (assuming that the disposition of these forces is the main causal factor in the civilians' choices; all other settings remain the same, but stochastic processes may account for at least some of the variation between runs).

\section{Threshold Response}

In this section we explore what effects different surge levels of coalition forces in the Ghazni region would have on the simulation, particularly how it would influence the number of soldier helpers. We ran this experiment with one set of settings from the experimental design changing only the size of the surge. Those setting are: Time Scale = 3; Social Theory = Representative; Influence $=$ On; Repression $=$ Off. Also, the number of CF and AGF at startup was 500 each, and the number of Taliban agents was set to 1000. To match our experimental design, we concentrated on the Ghazni region, i.e., the surge takes place in Ghazni. No agents were injected at each time step, so the Ghazni region had only its share of the initial soldier forces supplemented by the subsequent surge of coalition forces.

We started with a surge level of 40 and increased that by 10, up to 110. Each of these surge levels was run 60 times. We also ran three different mid-range surge levels per interval, 30 times each (i.e., we ran surge levels of 42, 45, 48 and 52, 55, 58, etc., up to 102, 105, and 108). The complex interactions and stochastic mechanisms inherent in the system produce a wide variance within each set of runs, and conclusions are thus difficult to ascertain. However, some trends do emerge as we graph the analysis. 
We defined a surge as successful if the number of soldier helpers at the end of the run was greater than the number of soldiers. Figure 4-3 is a plot of the number of runs that met the criterion for a successful surge. In this graph, the count results from runs of 30 repetitions are doubled to normalize with results from runs of 60 repetitions.

Figure 4-3. Results of surge experiment

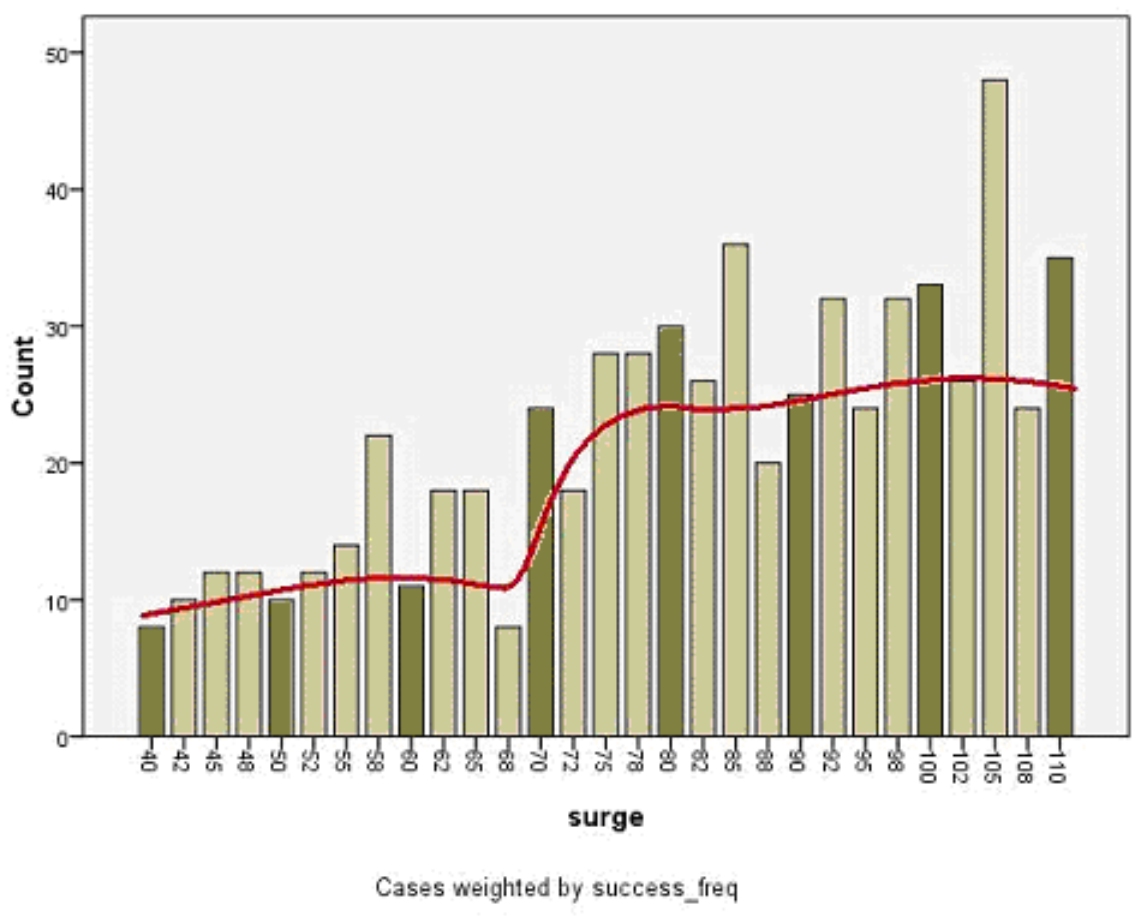

According to our results, when the surge level reaches approximately 70 soldiers, the number of successes increases dramatically. With a few exceptions, when the surge is less than 70, the number of successes tends to be around 10 to 15 out of every 60 runs; when the surge level is greater than 70, then the success rate tends to be 25 to 30 out of every 60 runs. Some threshold is apparently reached at the 70-soldier surge level that allows the system to greatly increase the success rate.

This is not the only threshold-type response we can see with this data. When one considers all the main surge values - 40, 50, 60, 70, 80, 90, 100, and 110 - in terms of how many soldier helpers exist at the end of each run, we can construct a histogram for each surge level and compare the results across the levels (Figure 4-4). 
Figure 4-4. Results as a function of run history

\section{D Graph 1}

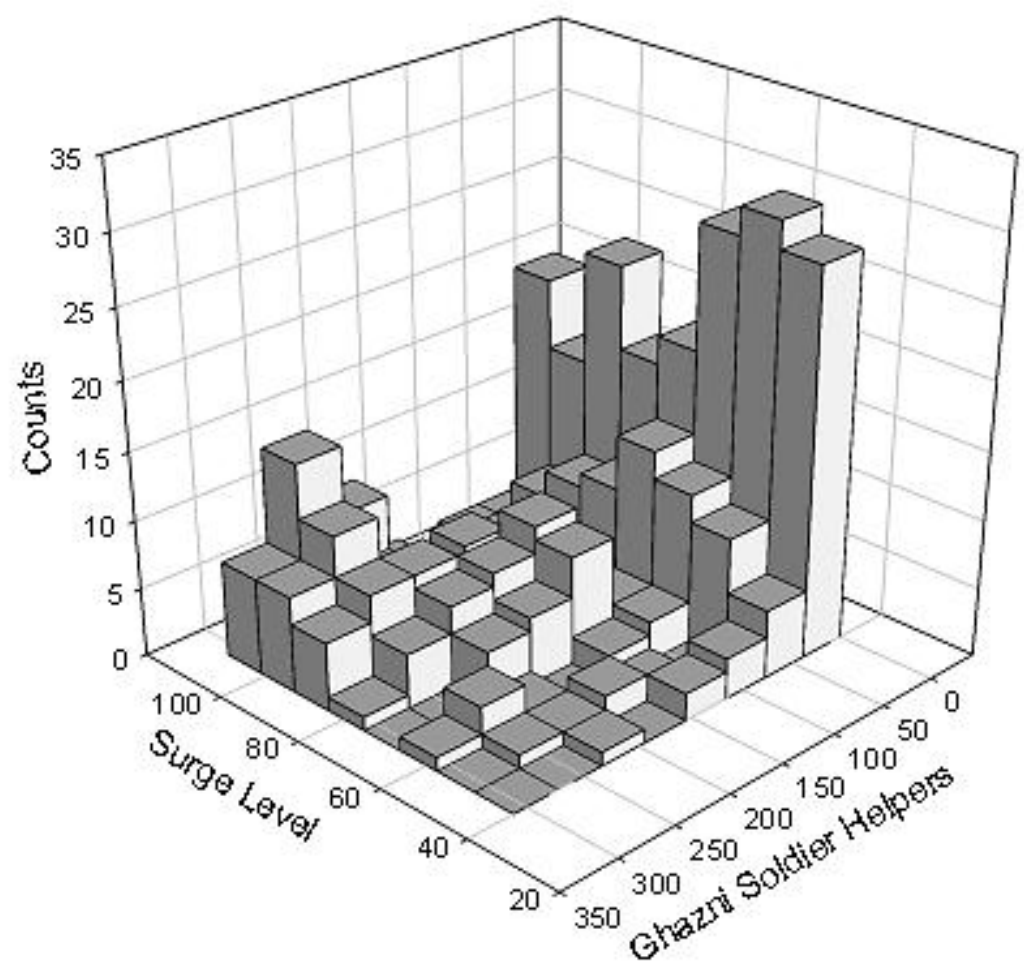

At a surge level of 40, most of the simulation runs result in somewhere between 0 and 40 soldier helpers in the Ghazni region, with very few runs resulting in more than 40 soldier helpers. The same is generally true for surge levels of 50 and 60. At surge levels of 70 and 80, far fewer runs end up with the number of soldier helpers in the $0-40$ range, and more runs fall in the 41-80 range. We can also see an increase in runs that result in 120300 soldier helpers. Finally, on the back left wall of the 3D histogram, we see that the highest surge levels usually end up with either a very low number of soldier helpers or a very high number of soldier helpers; rarely, do these surge levels result in a number of soldier helpers in the middle ranges.

In general, we can see that the trend pushes toward a greater and greater degree of separation between success and failure. At the higher surge levels, this threshold is more pronounced, resulting in either a low or a high number of soldier helpers, but rarely somewhere in between. 


\section{Evaluating Command Options for Ghazni in 2006}

Another set of experiments was used to evaluate COAs for the Ghazni region. For this set of simulation runs, we used the following settings that resulted from the calibration and validation procedures (using 2007 and 2005 data respectively) as outlined in Chapter 2.

Table 4-1. Experimental settings, Set 1

\begin{tabular}{|l|r|}
\multicolumn{1}{|c|}{ Parameter } & \multicolumn{1}{c|}{ Setting } \\
\hline influence & off \\
\hline social theory & legitimacy \\
\hline surge & 0 \\
\hline time scale & 3 \\
\hline
\end{tabular}

Repression was also turned on, not because it was significant, but because it had to have a setting, and turning repression on rather than off afforded a marginal (if not statistically significant) gain. We ran 60 replications for each of six combinations of the following settings:

Table 4-2. Experimental settings, Set 2

\begin{tabular}{|l|r|}
\hline \multicolumn{1}{|c|}{ Parameter } & \multicolumn{1}{c|}{ Setting } \\
\hline Economics & Off/On \\
\hline Surge Level & $0 / 200 / 400$ \\
\hline
\end{tabular}

The economics parameter is a refinement to the model that allows for nonmilitary intervention into the region. With economics on, the agent value for $\mathrm{E}+$ is changed from 300 to 1000, such that each agent in Ghazni will get an additional economic benefit for choosing a pro-government stance. The surge level parameter works as before, injecting new CF agents into Ghazni at time step 37. As this experiment is aimed at predicting the outcomes for these COAs during 2006, we used the following assassination data, taken from the WITS information for the Ghazni region:

- 3/18/2006 - 1 non-official public figure and 4 civilians killed in armed attack by suspected Taliban in Ghazni, Ghazni Province

- 5/21/2006 - 1 non official public figure kidnapped and killed, 1 civilian kidnapped by Taliban in Ghazni, Ghazni

- 5/25/2006 1 cleric kidnapped and killed in armed attack by suspected Taliban in Andar, Ghazni

- 6/15/2006 1 government Official kidnapped and killed, 2 civilians kidnapped by suspected Taliban in Qareh Bagh, Ghazni

- 7/05/2006 - 1 government employee kidnapped by Taliban in Ghazni, Ghazni 
The following table shows the time-step translation for these dates, using the time-scale of 3 days per simulation time step:

Table 4-3. Time-step translation

\begin{tabular}{|l|l|l|l|l|l|l|l|}
\hline & $3 / 18 / 06$ & $5 / 21 / 06$ & $5 / 25 / 06$ & $6 / 15 / 06$ & $7 / 05 / 06$ & Surge & End \\
\hline Time step & 1 & 22 & 23 & 30 & 37 & 37 & 62 \\
\hline
\end{tabular}

The resulting mindshare for these experiments is summarized in Figure 4-5, with the bars representing the number of runs (out of 60) that had a resulting mindshare for the Ghazni region within the indicated ranges:

Figure 4-5. Resulting Ghazni mindshare (a) without surge, (b) with surge set at 200, (c) with surge set at 400, (d) with economics turned off, and (e) with economics turned on

(a)

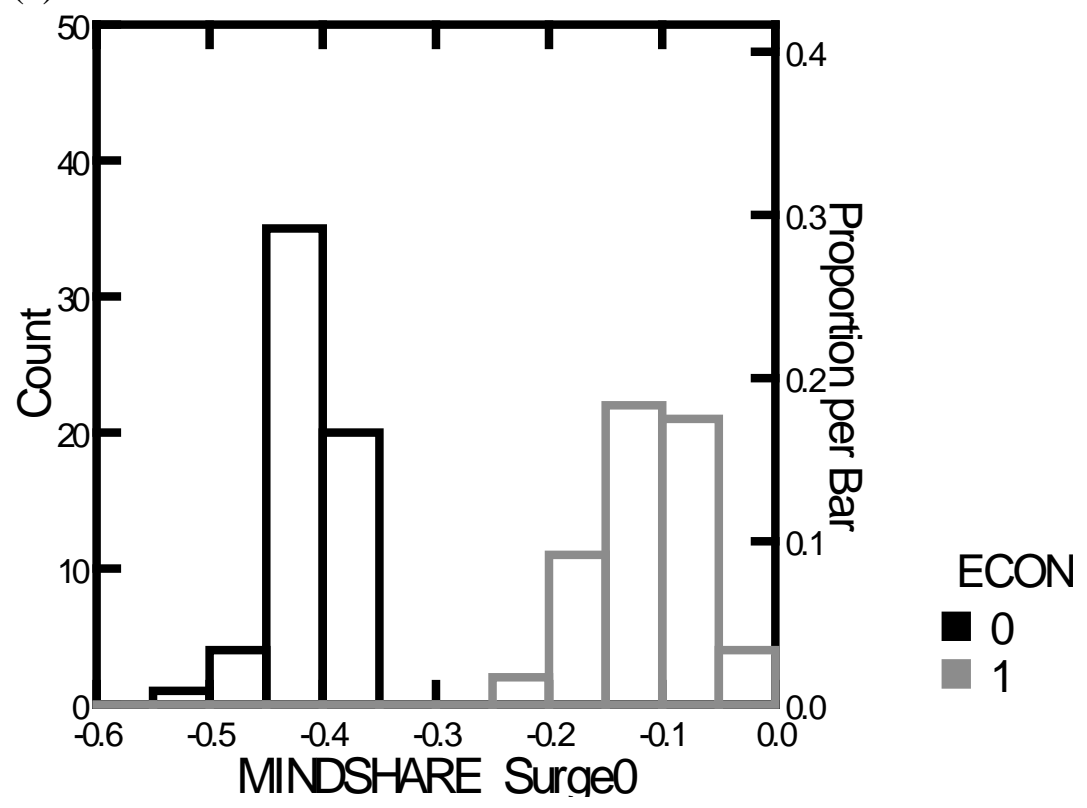


(b)

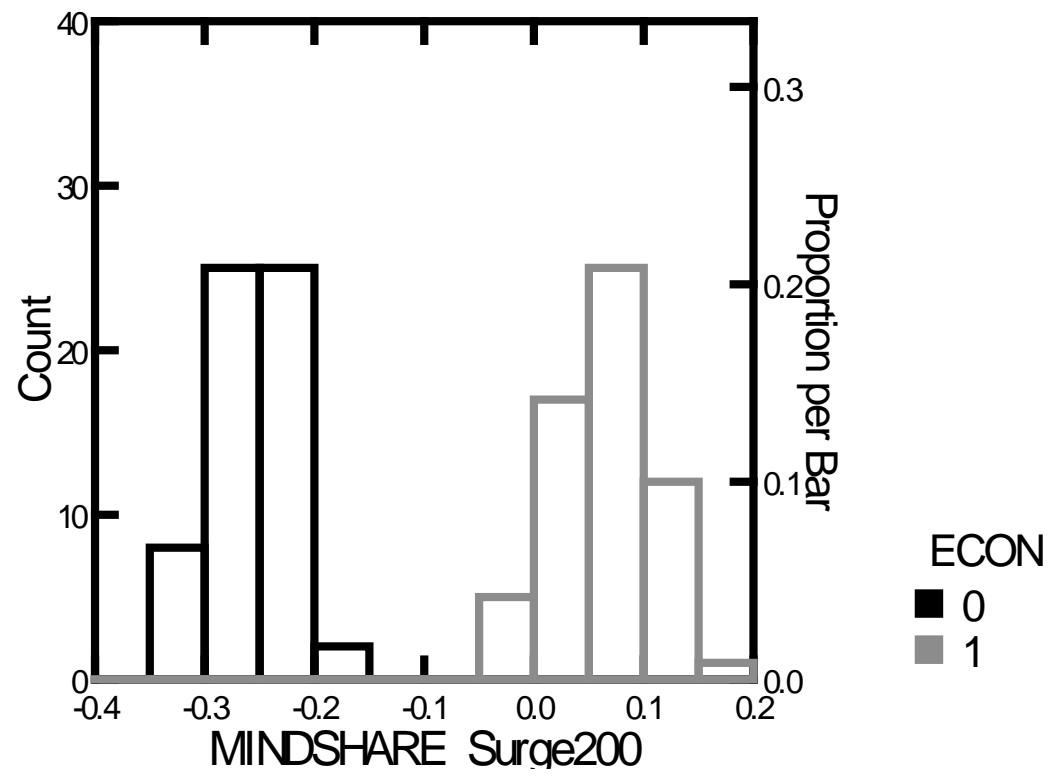

(c)

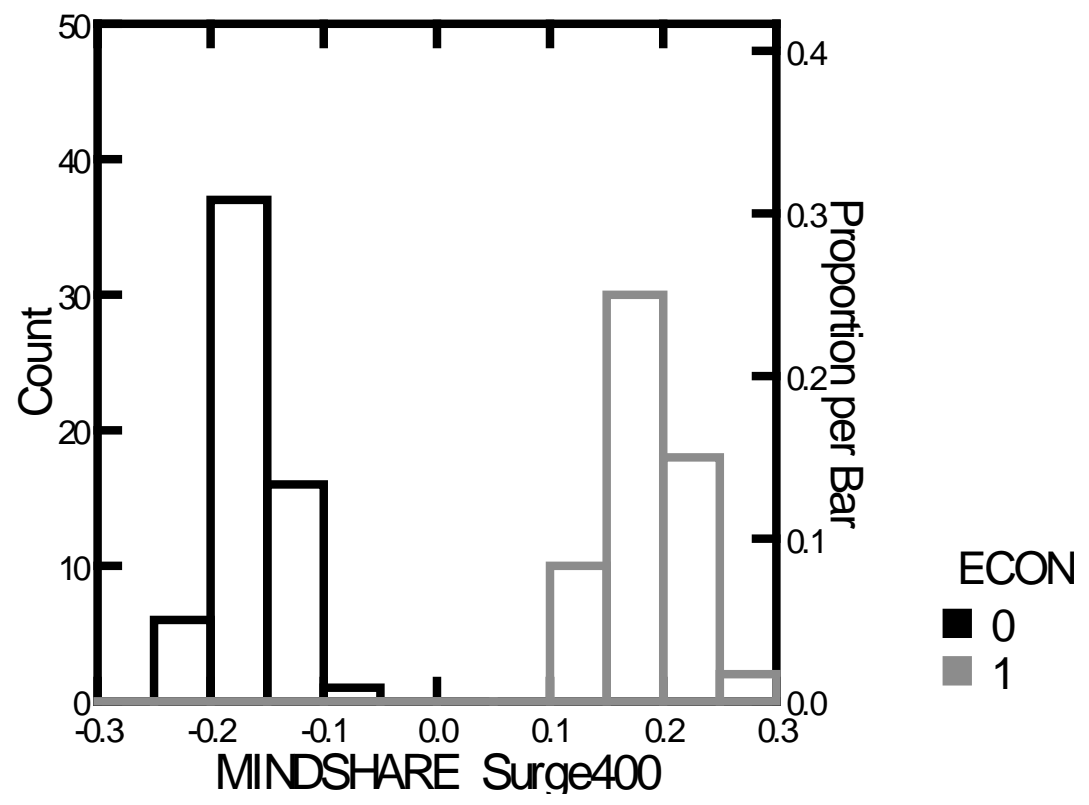


(d)

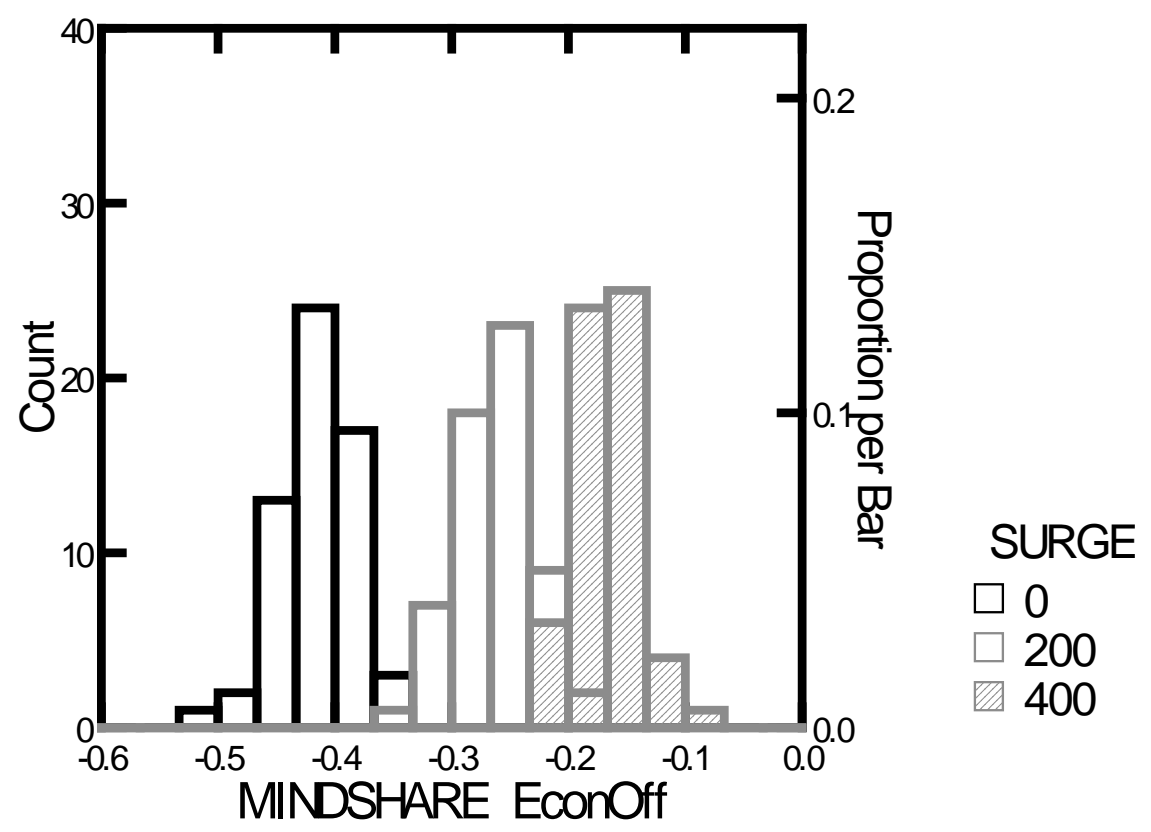

(e)

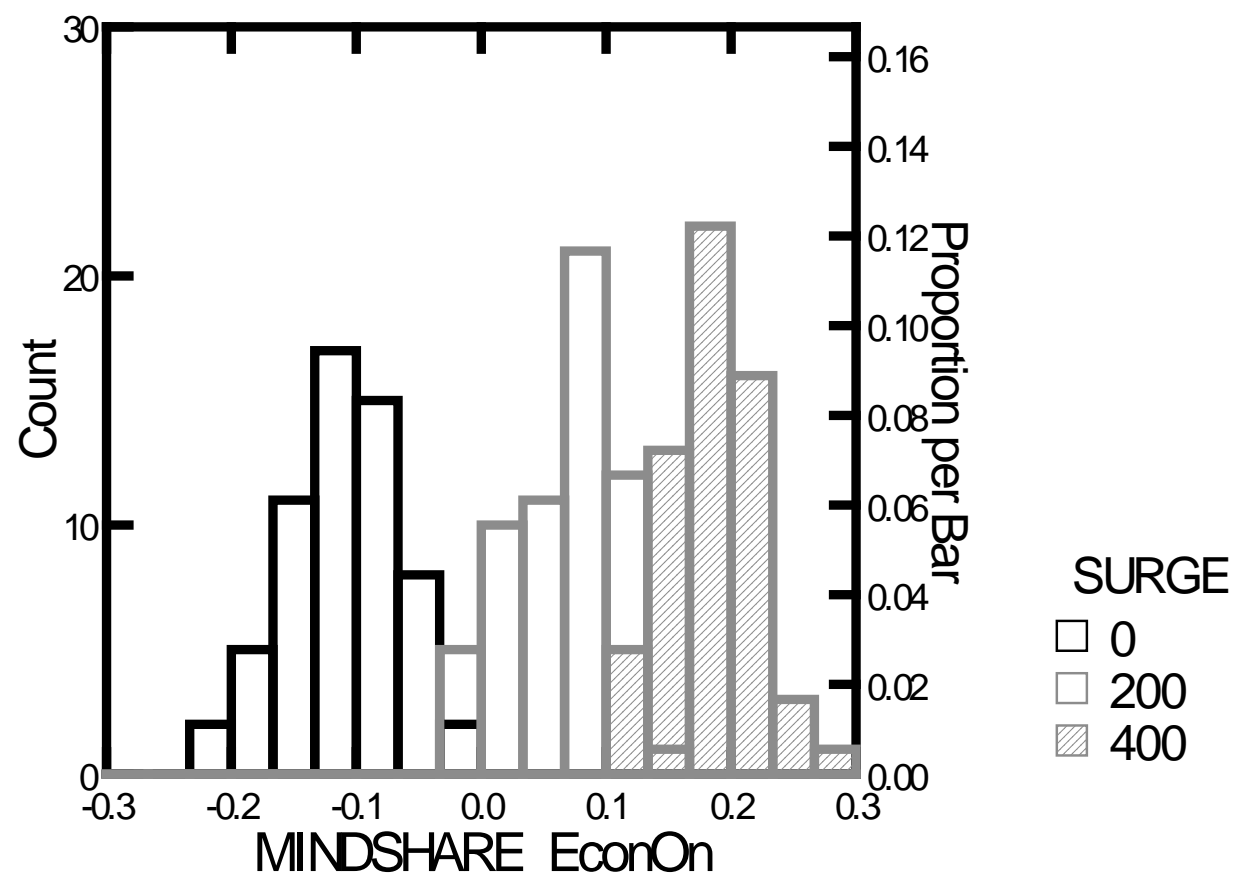

These experimental results show a significant correlation between (1) mindshare and surge level, and (2) mindshare and economics. Upon closer examination of these results, we can form two preliminary conclusions regarding our two COA parameters. First, even though a surge-as expected-does improve mindshare in the Ghazni region, we note 
that there are diminishing returns for surge levels. That is, the improvement in mindshare for the first $200 \mathrm{CF}$ agents is more than the subsequent improvement in mindshare for the second $200 \mathrm{CF}$ agents. (This holds true for both cases, i.e., whether economics is turned on or off.)

The second conclusion indicated by the data is that neither the economic incentives nor the troop surges are, by themselves, enough to guarantee that all 60 runs will result in an average mindshare for the Ghazni region that is above zero. In fact, only the combination of economics turned on and a troop surge of 400 is enough to meet this criterion.

Because the model does not currently include information about the cost of different interventions, it is not yet possible to make any cost-optimized strategy recommendations; the model will always favor sending in more troops and investing more money in Afghanistan. So while the base recommendation is that the United States is best advised to invest economically and militarily in Afghanistan, these recommendations are not tempered by the cost of the interventions and must be weighed accordingly. The general template of evaluating COAs using a simulation grounded in social science theory, however, remains in place as a valuable tool.

\section{Insights}

Within Chapter 4, we demonstrated the feasibility of explaining and predicting significant events in a complex environment, utilizing a social simulation such that command responses can be reasonably assessed in terms of their effectiveness for positively affecting outcomes. We have illustrated this by creating a CAS (complex adaptive system) model of Afghanistan on the order of 10,000 agents, each of which has its own rules and attributes that take into account social, economic, and military factors at the local level. Through the experiments detailed in this chapter we have identified two distinct types of surprising, or emergent, behavior, and we have used those results as a basis for evaluating various COAs. Appendix 8 discusses some of the issues involved in generating a natural-language narrative of these evaluations for presentation to nonexpert consumers of the model outputs. Appendix 9 demonstrates visual-analytic tools that not only allow for more direct manipulation of the model, but also help to extract and aggregate various outputs of the system for identifying causal relationships of the emergent behaviors. Finally, we have produced a distributive GA (genetic algorithm) that allows an automotive process for training the system based on real-world data.

In conjunction with the real-time update capability and automated calibration through a distributed GA (Chapter 3 and Chapter 2, respectively), this framework demonstrates the ability to create a realistic simulation that allows for testing and implementation of these social theories. The CAS model has demonstrated the emergent properties that constitute "surprise:" aggregate, nonlinear events that can be validated using data as identified in Chapter 1. Furthermore, we have created the visual-analytic tools to explore the state space of the model, which greatly facilitate identifying causal factors inherent in these surprising events, and we subsequently used these factors to make COA predictions for 2006 in the Ghazni region. 


\section{Chapter 5: Required Computational Complexity}

\section{Introduction}

The objective of Chapter 5 is to illustrate the feasibility of operating such a complex agent-based simulation system that can generate courses of action in computational times much less than 24 hours. The approach to this task included

- estimating the maximum hypothetical complexity of the agents that provides for each dimension of the HSBC, incorporating elements of modern social theory;

- characterizing the maximum complexity of the environment that allows for realtime updating of the environment with unfolding events;

- developing literature examples of analogous systems of comparable complexity;

- comparing employed computational resources to those currently available with similar run times for case studies.

Disaggregated models that also track the motion of individual social atoms are computationally intensive. The complexity of moving resources and people is compounded by increasing granularity in data that allows inclusion of finer behavioral dynamics, thereby increasing the computational intensity. Given that dynamic planning and operational support require large-scale simulations within narrow time windows, reducing execution times is the most critical challenge and can be met only with significant computing resources beyond desktop environments. High-performance computing is, therefore, a necessary enabler for simulations that generate multiple courses of action for a large social region.

Testing the 31-million-agent model of Afghanistan's population demonstrated the ability to computationally control and analytically manipulate a system with the large number of agents (i.e., $10^{8}$ ) necessary to model regions at the individual level. Simulations in computationally tractable times can be performed with computational costs that range from less than a dollar for simulations with simple interactions to a few hundred dollars for the largest and most complex simulations.

The current generation of societal modeling and simulation uses existing architectures that are based on linking independently developed models to predict effects resulting from specific triggering events. These systems are characterized by the following attributes:

- They are modular systems with each module (model) optimizing one or two elements of the HSBC domain. Multiple models are then linked together to cover the HSBC spectrum. The degree of aggregation in the models does not permit sensitivity analysis with respect to the causes of surprising changes in human behavioral dynamics without prohibitive computational power or analytical time. 
- Current agent-based models allow the agents to interact within their environment without constraints. The models generate a large number of potential futures but fail to quantify the probability of any single stream of future events. They incorporate little or no modern social scientific theory or analysis to adjust the rule base to mimic real-world observations. To generate realistic behaviors consistent with real-world observations, the entire range of HSBC theories must be leveraged to inform the simulation rule sets.

- These systems employ a flexible architecture to combine models from a variety of constituencies, thereby permitting a community (M\&S community, alliance community, interagency coordination and coalitions) to jointly experiment and understand intracommunity interactions. However, current models and simulations provide no capability for real-time or near real-time data updates as surprising events unfold or rapid changes occur in societal dynamics.

- These systems cannot conduct a sensitivity study of key causal effects of an observed change in a region's stability in reasonable computing times. They provide only limited capability for analysts to select streams of future events important for the question at hand. Current systems are difficult for analysts to intuitively comprehend and easily control.

Many recent advances permit us to consider the next generation of modeling and simulation systems that can support the ability to anticipate adverse human behavioral developments and that can evaluate alternative courses of action to mitigate the impact of these developments on U.S. national security interests. They include

- new approaches to multi-agent systems that allow us to envision goal-oriented agents and event responses that span the HSBC domain, as well as modern frameworks and architectures that support models using agents that obey rule sets informed by modern social theory;

- advances in high-performance computing (HPC) resources that can assist computationally intensive cases when large numbers of agents are required to adequately describe a regional stability and when aggregation obscures causal factors;

- emergence of architectures that can handle $10^{8}$ agents, disaggregated to the level of individuals behaving according to modern social theories within community scales important to regional stability, while still providing useful predictions in computationally tractable times.

This research thrust has as its central goal the ability to simulate population behavior in a way that anticipates adverse future developments and supports alternative course of action assessments to mitigate them. The objective of the proposed research is to develop the next generation of social simulations that integrates advances in social science theory and computational sciences. This new generation of societal simulation will be characterized by the following attributes:

- $\quad$ These systems will be independent of the linkage of separate models (i.e., a political model linked to an economic model, linked to an infrastructure model, etc.) that are limited by aggregation and by unrealistic behavior rule sets. 
- The new systems will be able to conduct sensitivity studies of key causal effects of an observed change in a region's stability (surprises) in reasonable computing times.

- These systems will allow analysts to intuitively comprehend and control the simulation of future event streams and will facilitate analysis of historical precursors.

The objective of this chapter is to provide a summary of the insights from the current effort to (1) demonstrate or document the ability to computationally control and analytically manipulate a system with the large number of agents (i.e., $10^{8}$ ) necessary to model regions at the level of the individual agent and (2) complete "what if" scenarios with clock times of much less than one day. The deliverable for this task included a literature survey of systems of comparable complexity or systems that can be extended to the size of systems required by the ultimate ACSES program.

\section{Addressing the Computational Complexity Issue}

The granularity of the data required to describe HSBC factors important to regional stability places stringent constraints on the computational system. Description of most geographic regions will require on the order of $10^{8}$ agents with complexity assumptions dictated by the richness of the data processing underlying the HSBC theoretical framework.

The objective of Task 5 is to illustrate the feasibility of operating such a complex agentbased simulation system that can generate courses of action in computational times much less than 24 hours. Because the simulation results are required to be actionable, longer computation times may not allow the simulation to execute within the commander's decision cycle. The approach to this task included

- estimating the maximum hypothetical complexity of the agents that provides for each dimension of the HSBC, incorporating elements of modern social theory;

- characterizing the maximum complexity of the environment that allows for realtime updating of the environment with unfolding events;

- developing literature examples of analogous systems of comparable complexity;

- comparing employed computational resources to those currently available with similar run times for case studies.

Within the literature, three important studies have described the construction of agent systems as well as construction of those systems needed to manipulate more than 100 million agents. The first system study (Germann et al.) modeled the propagation of avian influenza. Germann's model distributed a synthetic population of 281 million individuals among 65,334 census tracts to closely represent the actual population distribution according to publicly available 2000 U.S. census data. The second study also modeled the global transmission of avian influenza. The model, MIDAS, used 300 million agents, but these agents were simpler in design than the Germann agent. The third study, undertaken at the Brookings Center on Social and Economic Dynamics (CSED), reported 
development of a flexible, large-scale, distributed agent-based model that is capable of simulating up to four billion agents.

The basic agent-based computational model (ABM), unique for its scale and adaptability, has a 300-million-agent implementation specific to the United States. This spatially and demographically realistic implementation is based on the 2000 U.S. census data, contains a $4000 \times 4000$ travel matrix (encoding a Gravity Model), and is resolved at the level of the census track. This model, named the U.S. National Model, was developed as a tool for applied public policy research.

The ABM methodology was successfully applied to a wide variety of policy questions, including infectious disease transmission, public health challenges such as obesity and smoking, and civil violence. In brief, these "virtual societies" are typically characterized by the following:

1. Explicit space: Events transpire on an explicit space, such as an n-dimensional lattice, a social network, an organization, or, as in the U.S. National Model, an explicit map.

2. Heterogeneous autonomous individuals: Every single agent is explicitly modeled and tracked. They each differ in myriad ways - genetically, culturally, by social network, by their rules of behavior, by health status - all of which may evolve or adapt endogenously over time depending on their autonomous interactions with other agents and with their environment.

3. Local interactions: The traditional assumption of mass action kinetics is eschewed in favor of a more realistic depiction of local social contact dynamics in discrete and explicitly represented social units (the home, the workplace, etc.).

4. Bounded rationality: Unlike most traditional models, individual agents do not have perfect information, and they do not optimize. As with real people, they rely on simple heuristics, community norms, and biased and partial reporting in their decision-making.

Agent-models generate extremely high volumes of clean data that are analyzed statistically to yield very high-fidelity characterizations of their behavior, sensitivities, bifurcation points, etc. This innovative technique is particularly well suited to applied public policy research for three reasons: it allows rich and diverse human behaviors to be incorporated rigorously, it facilitates interdisciplinary research and stakeholder participation by providing an easily communicated common framework, and the computational environment provides a test-bed for public policy interventions that is both timely and cost-efficient.

The agent-based U.S. National Model now on an unprecedented scale allows analysis of the complex interactions between a particular policy and the environment that it is intended to affect. While the model is currently being used to design and test optimal policies to mitigate and contain an influenza pandemic in the United States, research 
teams continue to expand the core model's functionality with the goal of creating a spatially and demographically realistic global agent-based model.

Although at least three systems exist that provide applicable benchmarks, this calculation will compare the hypothetical ACSES system to the Germann system to provide a measure of the computational resources required to manipulate the model. Therefore, all comparisons associated with this task will benchmark against the Germann agents and simulation system.

The conceptual layout of the generic ACSES agent is shown in Figure 5-1.

Figure 5-1. The conceptual design of the agent is built with seven conceptual modules

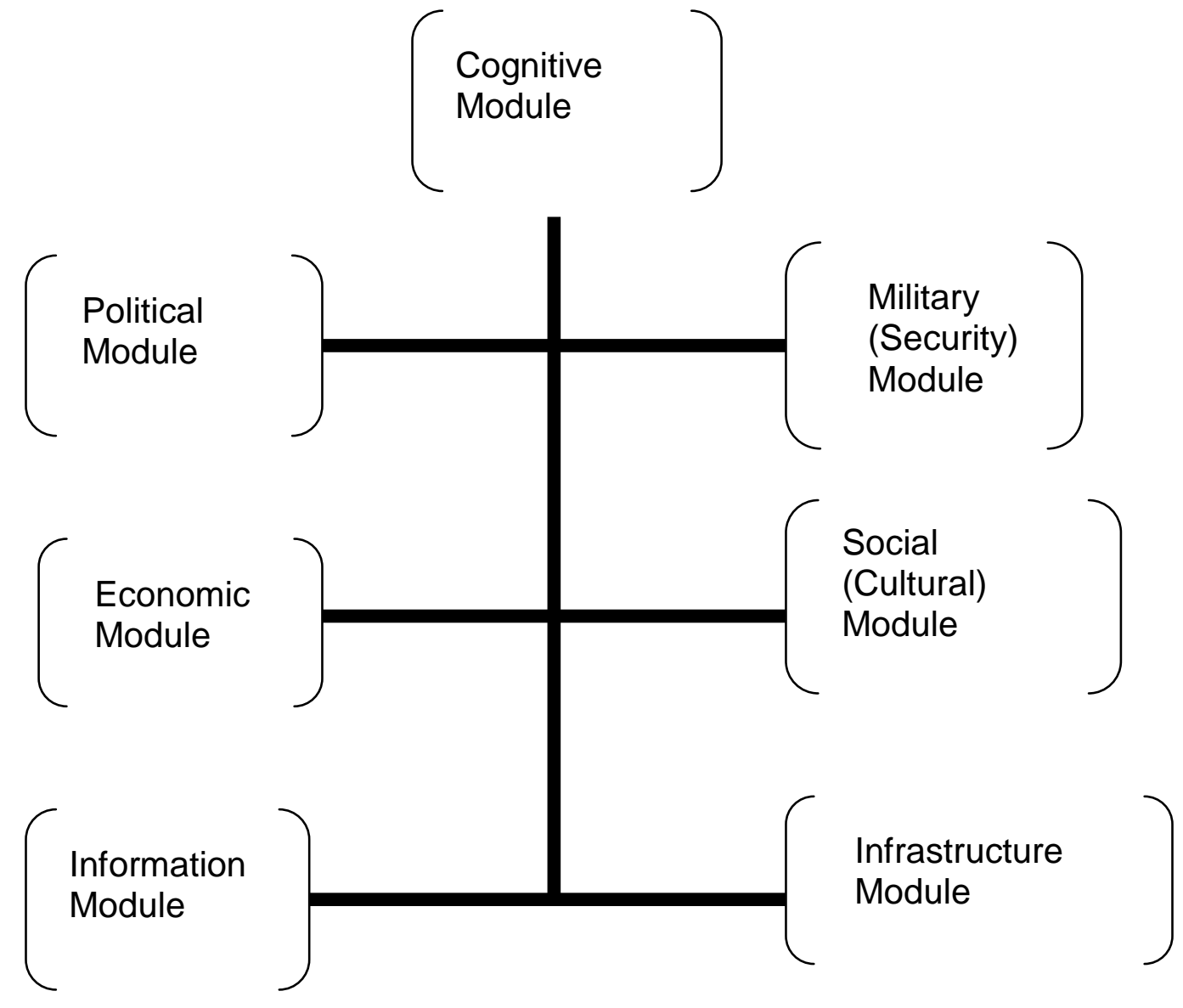

Because the purpose of the individual modules (excluding the cognitive module) is to provide a flexible framework to test emerging social theories, it is not possible to calculate the computational requirements of each module yet. However, the size of the individual modules must be large enough to ingest information and provide a metric to the cognitive module so that the agent can evaluate whether a change of state is to occur. As each of the six other modules receive inputs from sensing their environment, the 
algorithms embedded in the cognitive module will calculate the agents' change in state based upon this newly received information. Modern theories will inform the algorithms within each module.

The cognitive module represents the controller of the agent and after receiving the metrics from each of the six other modules, “decides” whether the agent undergoes a change in state. How complex each individual agent (and in turn, each of the seven modules) must be to actually generate these metrics is an on-going topic of research. However, the comparison of emerging computational capabilities will require a hypothesized estimate of the computational size of the seven modules.

To scale the computational requirements, we will assume an enhanced agent with maximized complexity based on the PMFServ design of Silverman et al. This hypothetical agent design assumes we use modules that reflect elements of modern social theory and is sufficiently flexible that current and emerging theories can be tested without rebuilding the system from first principles. We will assume this hypothetical-design-basis agent will have the modules based on an ontology of current modern social theory in each of the political, military, economic, social, information, and infrastructure (PMESII) domains as well as a cognitive module for balancing and trade-offs so that the agent may take action depending upon its updated environment. We will then compare this modified Silverman-style agent to the Germann agent to estimate the ACSES system's computational requirements.

The agent used in the Germann study is shown in Figure 5-2. This agent is much simpler than those expected to be required for the ACSES system. Although the system provides for a synthesized population operating according to information received in a relatively sophisticated manner, the framework envisioned below does not provide for trade-offs between the various domains, such as the six domains in the ACSES design. One would expect that Germann's model and the ACSES's political, economic, military, etc. modules would be of similar complexity. Therefore, one can imagine an ACSES agent comprising the Germann model reproduced six times plus a cognitive model (to provide the trade-offs among the six domains) that is at least as complex as any of the other modules. The computational intensity is that required for an agent consisting of the seven modules (cognitive plus six elements). 
Figure 5-2. Germann model notional architecture

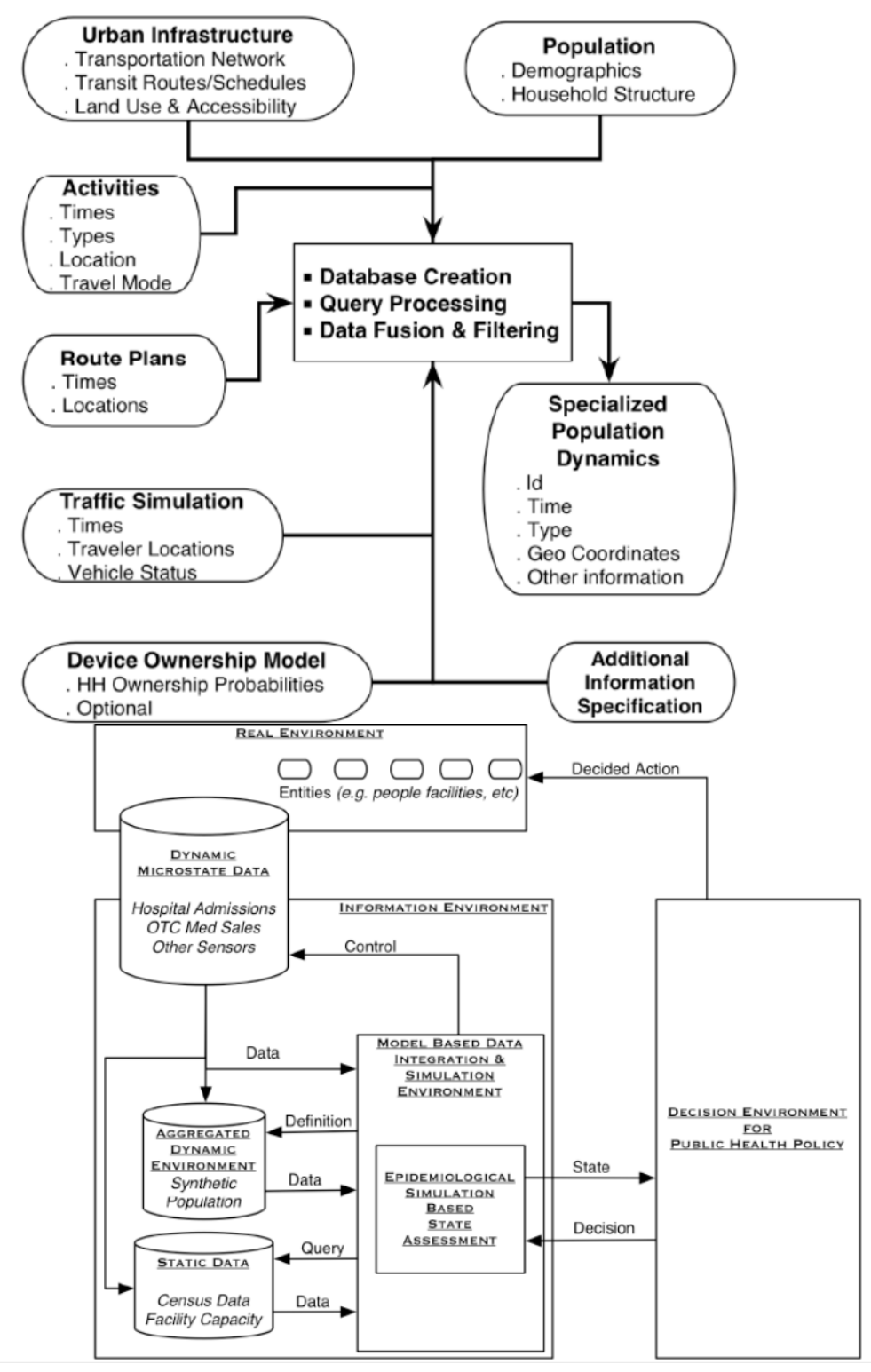

Starting with the cognitive module, Silverman et al. created goal, standards, and preferences (GSP) trees that hold the values that compose the agent's cognitive (or emotion) module and applies the hierarchy to evaluate the state of the world and the actions of others. They also are the motivators for the agent's own actions and give rise to event stress (failed values) as well as subjective utilities for next action choices. One wants to design the GSP trees so that each possible action in the scenario will impact some branch of one or more of the GSP trees. GSP tree examples are shown in Figure 53. 
Figure 5-3. Example goal, standard, and preference tree

\begin{tabular}{|c|c|c|}
\hline Som_Civilian_Female & Som_Civilian_Male & Som_Militant \\
\hline 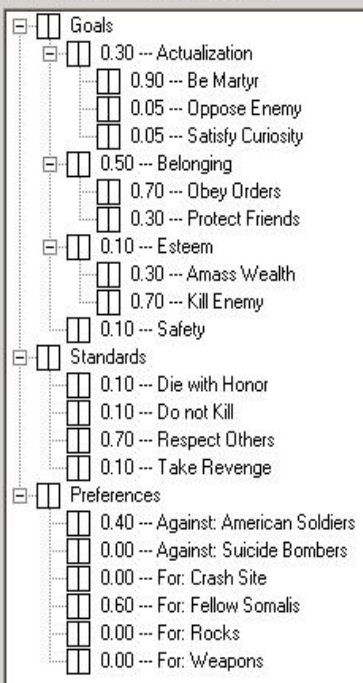 & 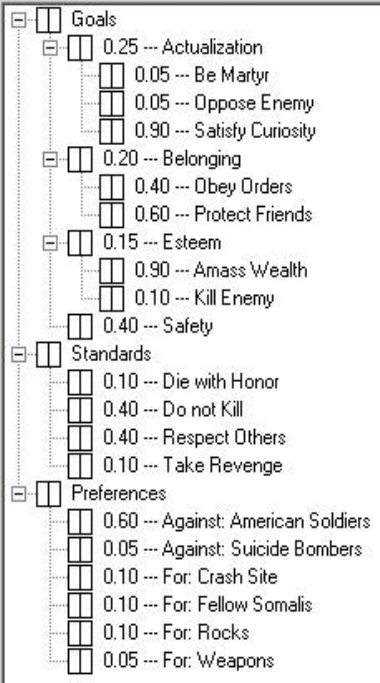 & 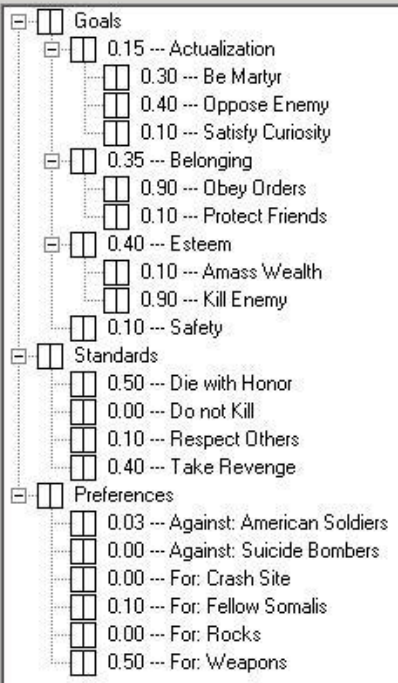 \\
\hline
\end{tabular}

Once the GSP tree structure is settled, one begins the process of assessing how important each branch is to a given archetype in the scenario. Empirical information is organized into evidence tables through a modified content analysis process by breaking statements into simpler units with one theme (replicating statements when necessary), adding additional fields, namely reliability and relevance, and then sorting.

Although the Silverman model above reflects only one of a number of possibilities as far as a cognitive module based on modern social theory, most cognitive modules are composed of some hierarchy upon which the metrics from the other six modules are weighted and evaluated against criteria for a transition of state. However, if we compare the agents used in the ACSES modules to those used in the Germann study, the complexity of any one of the seven modules would be on the same order as the complexity of the agents Germann used. In the Germann study, the agents were programmed to move and interact with their fellow agents according to observed population mobility models, but in reality, the agents were simple state machines.

Germann's simulation of pandemic flu propagation employed a model population of 281 million individuals distributed among 65,334 census tracts to closely represent the actual population distribution according to publicly available 2000 U.S. census data. Each tract was in turn organized into 2000-person communities. The model ran in cycles of two 12hour periods ("day" and "night"), during which individuals would associate within seven contexts ("mixing groups"). In six of these contexts (households, household clusters, preschools, playgroups, schools, and work groups), relatively close person-to-person association regularly occurs. Additionally, "neighborhoods" and "communities" provide unspecified contexts (e.g., shopping malls) within which occasional casual person-to- 
person association occurs. Daytime contacts occur in neighborhoods and communities as well as in the age-appropriate setting, and nighttime contacts occur only in households, household clusters, neighborhoods, and communities. U.S. census data on tract-to-tract worker flow is used to model the commute of working adults to their workplaces, thus accurately capturing the short- to medium-distance population mobility important for disease spread. In addition, each individual took occasional long-distance trips (three per year on average), lasting between 1 day and 3 weeks (4.1 days on average), matching Bureau of Transportation statistics.

Germann's simple model of long-range travel can be extended to account for different types of travel (e.g., business or leisure) or groups of travelers (such as a family) or to explicitly incorporate the airline network structure.

To model the introduction of pandemic influenza into the United States, Germann assumed that impenetrable borders are either prohibitively expensive or impossible to create, and that international air travel is the dominant mode of influenza introduction from outside the United States. Consequently, a small random number of incubating individuals, equivalent to $0.04 \%$ of arriving international passengers, is introduced each day at each of 14 major international airports in the continental United States. The simulation covered 180 days, roughly the length of a U.S. influenza season. We assume that, because of the uncertainty in diagnosis of influenza infections and the sporadic nature of the early stages of an outbreak, a cumulative number of 10,000 symptomatic individuals nationwide is required to trigger a nationwide pandemic alert.

Similar to Germann's approach, the ACSES's Afghanistan example starts with the distribution of a model population. The Afghanistan population in 2003 was estimated by the United Nations at 23,897,000. In that year approximately $3 \%$ of the population was over 65 years of age, with another $43 \%$ of the population under 15 years of age. The country had 107 males for every 100 females in 2003. According to the UN, the annual population growth rate for 2000-2005 was 3.88\%, with the projected population for the year 2015 at 35,473,000. The population density in 2002 was 42 per sq km (110 per sq $\mathrm{mi})$.

The Population Reference Bureau estimated that 22\% of the population lived in urban areas in 2001. The capital city, Kabul, had a population of 2,454,000 in that year. Other major population centers included Kandahar, 339,200; Mazār-e Sharif, 239,800; and Herat, 166,600. According to the United Nations, the urban population growth rate for 2000-2005 was 6.9\%. These figures are unreliable, however, because many city dwellers have left their urban homes for refuge in rural areas. Approximately $20 \%$ of the population is nomadic.

Afghanistan spans a geographic area of 647,500 square kilometers. As documented within Landscan Global, the population can be distributed among $1 \mathrm{sq} \mathrm{km}$ sections, and the nomadic population can be distributed based on historic migratory patterns. The number of agents per section, or district, varies from $10^{5}$ in Kabul to less than 20 in the outlying mountainous areas. 
Rather than the seven mixing groups assumed for the Germann example, we expect that the Afghanistan example will need approximately 22 contexts, or mixing groups, to describe how individuals can associate with and influence each other.

Although each individual may interact with any member of his or her mixing group, to assume that everyone within a mixing group interacts with every member within the country is an unreasonable aggregation when estimating the maximum complexity necessary to explain causes of regional stability. Communication is neither instantaneous across regions nor received in a uniform manner within the community. The maximum number of communities can be represented by 650,000 census tracks multiplied by the 22 mixing groups.

The table below compares the complexity of the hypothesized system with the complexity of the Germann system. As illustrated in this table, one would expect that the hypothesized system would be approximately 7 times more complex than the Germann system. If we assume quadratic scaling of the computational requirements, then the number of CPU years required to run a ACSES simulation is $7^{2}$ or 49 times that of a simulation using the Germann system.

Table 5-1. Complexity comparison between ACSES and Germann simulation systems

\begin{tabular}{|l|c|c|c|c|}
\hline System & $\begin{array}{c}\text { Number of } \\
\text { agents }\end{array}$ & Census tracts & $\begin{array}{c}\text { Mixing groups } \\
\text { per tract }\end{array}$ & $\begin{array}{c}\text { Number of } \\
\text { modules per } \\
\text { agent }\end{array}$ \\
\hline Germann et al. & $2.8 \times 10^{8}$ & $6.5 \times 10^{4}$ & 7 & 1 \\
\hline $\begin{array}{l}\text { Hypothesized } \\
\text { ACSES system }\end{array}$ & $2.4 \times 10^{7}$ & $6.5 \times 10^{5}$ & 22 & 7 \\
\hline
\end{tabular}

A typical production run as reported by Germann was performed on 256 central processing units (CPUs) of a 2048-processor Intel Xeon 2.4-GHz cluster with Myrinet interconnect. A typical computational run took 8-12 h to complete a simulation of 180 days. In all $\sim 200$ production runs were performed, amounting to a cumulative $\sim 70 \mathrm{CPU}$ years of computer time. On average, a Germann production run would require about 125 CPU days (70 CPU years $\times 365$ days per year/200 runs) to complete. The computational time estimate is approximately 6250 CPU days for running a single "what if” scenario. This assumes the ACSES agent is approximately 50 times more computationally complex than the Germann agent.

To compare this value to existing computational resources available at ORNL, we consider the Cray XT4 named Jaguar comprising 124 cabinets containing more than 11,700 dual-core AMD processors. The system achieved 101.7 teraflops on the Linpack benchmark, which is more than 85 percent of its theoretical peak of 119 teraflops. By this calculation, a scenario would require approximately 12.8 hours to complete.

Jaguar has at least one more upgrade in its future. That upgrade will more than double Jaguar's performance to a peak of 250 teraflops. By late 2008, a new one petaflops Cray 
'Baker' system will be installed. Both machines will employ the upcoming quad-core AMD Opteron processors. If one petaflop machine could be dedicated to this effort, then a typical scenario would be calculated in about 75 minutes.

Before the impending upgrade, the existing Jaguar system would be able to perform the expected ACSES simulations in clock times significantly less than one day. The actual time that would be required would depend on the actual agent design, the time period within the simulation necessary to observe emergent behavior, and the overheads associated with these simulations.

\section{Computational Costs}

Fiscal feasibility can be assessed not only from the estimated computational time of a maximum-size, 100-million-agent system but also from the cost of individual simulations. Surveying rental costs of HPC systems, IBM and Sun Systems have quoted 1024 processors racks on Blue-Gene-type systems (similar in capability to the Jaguar system) that can be rented on the basis of only those processors actually used and the time they are engaged. Although lower costs are usually negotiated, most bids cluster around one dollar per processor hour. At this rate, the simulation of 31 million agents (approximate 2006 population of Afghanistan) would require approximately $\$ 0.08$ per simulation time step.

To illustrate the maximum computational time and costs, a calculation was performed for the most complex agent envisioned for the ACSES system. Renting individual racks and paying only for those processors actually used, each of the most complex scenarios would require approximately $\$ 1250$ in computational time for a 1000-time-step simulation. A simulation with 3-day time steps for six months would cost on the order of $\$ 75$. Even at the extreme end of computational intensity, these costs are well within the costs of similar simulation programs currently under development.

Between these two extremes is a three-dimensional trade space that trades speed of simulation, simulation costs, and observed emergent behavior. Compromises to allow the synthetic populations to fit into existing computational platforms are no longer mandatory.

The lesson learned from this calculation is that compromises taken in the past to aggregate populations because small platforms were employed to perform social simulations can be eased through HPC applications. These applications will expand the opportunity to leverage human terrain results within a simulation environment and will offer new options for strategic planners.

Disaggregated models that also track the motion of individual social atoms are computationally intensive. The complexity of moving resources and people is compounded by increasing granularity in data that allows inclusion of finer behavioral dynamics, thereby increasing the computational intensity. Given that dynamic planning and operational support require large-scale simulations within narrow time windows, 
reducing execution times is the most critical challenge and can now be met with significant computing resources beyond desktop environments. High-performance computing is, therefore, a necessary enabler for simulations that generate multiple courses of action for a large social region.

The resulting insight is that HSBC simulations are an HPC computing problem in the same sense that weather prediction is an HPC problem; the computations can be done in hours or days (not months, like some "big physics" problems), but the need to meet an "actionable" deadline imposes a completion time of minutes or hours and, consequently, a need for HPC. The cost and availability of these resources have now placed these resources within the reach of the combatant commands.

\section{Simulation Setup of the ORMAC System}

Although the feasibility of performing disaggregated simulations has been demonstrated, the benefits of disaggregation in forecasting outcomes are not well-defined. An experiment was performed to gain some insights about the additional information available to commanders from disaggregated simulations. Two independent simulation systems ran a simple Afghanistan example using leadership theory at different levels of aggregation. A NetLogo system used about $10^{4}$ agents, and an ORMAC simulation used approximately 31 million agents. Although the synthetic populations and the underlying theories were identical in the two systems, minor adaptations to the NetLogo simplifying assumptions were used to operate at the disaggregated level of the individual. The following section provides a description of the ORMAC adaptations to the NetLogo simulation implementation and synthetic population data generation.

\section{Summary of the Adaptation of the Social Theories for the Comparison Experiment}

Three categories of social theories have been proposed for exploration by this research effort: leadership, learning, and social mobilization. Here, we will focus only on the computational aspects of these theories.

Although the implication and interpretation of the leadership theories and the social mobilization theories are different, their computational implementation can be achieved by using a single utility function:

$$
U=(1-L)^{w_{L}}(1-C)^{w_{C}}(1-I)^{w_{I}}(1-E)^{w_{E}}(1-V)^{w_{V}}(1-F)^{w_{F}}(1-R)^{w_{R}} .
$$

This utility function encompasses seven factors: $L$ is the agent's loyalty to the leader, $C$ is the coercion factor, $I$ is ideology, $E$ is economic welfare, $V$ is security against violence, $F$ is the influence of close associates, and $R$ is repression and social influence for defying repression. $L, C, I, E, V, F$, and $R$ fall in the range [0,1]. The weights are required to be nonnegative, to be less than or equal to 1 , and to sum to $1: w_{L}+w_{C}+w_{I}+w_{E}+w_{V}+w_{F}$ $+w_{R}=1$. The overall computational goal is to identify the behavior value (B) that would allow a citizen agent to maximize the value of its utility function. 
Let $\mathrm{O}$ denote the order of the leader $(+1$ : pro-government, 0 : neutral, and -1 : proTaliban), B denote the behavior of a citizen agent (+1: pro-government, 0 : neutral, and 1: pro-Taliban), $\bar{B}$ denote the average behavior of the citizen agents within a certain radius of the focal agent ( $\bar{B} \in[-1,1]$ ), $\alpha$ denote the relative magnitude of the resources that can be brought by the leader to bear against an agent ( $\alpha \in[0,1]$ ), and $\mathrm{P}$ denote the position of a citizen agent ( $P \in[-1,1])$. Eq. (1) can be rewritten as the following:

$$
\begin{aligned}
& U= \\
& {[1-|(O-B)| / 2]^{w_{L}}} \\
& {[1-\alpha \times|(O-B)| / 2]^{w_{C}}} \\
& {[1-|(P-B)| / 2]^{w_{I}}} \\
& (1-E)^{w_{E}} \\
& (1-V)^{w_{V}} \\
& {[1-\mid(B-\bar{B}) / 2]^{w_{F}}} \\
& (1-R)^{w_{R}}
\end{aligned}
$$

The effect of any of the seven factors in Eq. (2) can be turned off by setting the associated weight to 0 . When $w_{F}$ and $w_{R}$ are set to 0 , this utility function reflects the leadership theories. The lack of theories providing guidance for simulating the behavior of armed forces and their mobility patterns has led to the decision of disabling the repression factor $(\mathrm{R})$ by setting $w_{R}$ to 0 in our current simulation.

Learning theories were introduced to demonstrate potential changes in a citizen agent's ideology and loyalty over time. Incorporating learning theories into Eq. (2), we obtain the following new utility function:

$$
\begin{aligned}
& U(t)=\left[1-\lambda_{i}(t) \times\left|\left(O_{i}(t)-B_{i}(t)\right)\right| / 2\right]^{w_{L}} \\
& {\left[1-\alpha_{i} \times\left|\left(O_{i}(t)-B_{i}(t)\right)\right| / 2\right]^{w_{C}}} \\
& {\left[1-\left|\left(P_{i}(t)-B_{i}(t)\right)\right| / 2\right]^{w_{I}}} \\
& \left(1-E_{i}(t)\right)^{w_{E}} \\
& \left(1-V_{i}(t)\right)^{w_{V}} \\
& {\left[1-\left|\left(B_{i}(t)-\bar{B}_{i}(t-1)\right)\right| / 2\right]^{w_{F}}} \\
& \left(1-R_{i}\right)^{w_{R}}
\end{aligned}
$$

where $\lambda$ is a citizen agent's perception of his leader's legitimacy $(\lambda \in[0,1])$. The notation "variable $(t)$ " is interpreted as "the value of variable of citizen agent $i$ at time $t$." 
For instance, $\bar{B}_{i}(t-1)$ refers to the average behavior value in citizen agent $i$ 's territory at time $(t-1)$.

The four learning theories are cognitive dissonance, results-based, homophily, and socialization (no changes over time). These theories govern parameters $\lambda_{i}(t)$ and $P_{i}(t)$. More details on learning theories can be found in Chapter 2: Agent-Based Modeling. Based on data source availability, we implemented the cognitive dissonance and the homophily theories.

If the cognitive dissonance theory is applied, $\lambda_{i}(t)$ and $P_{i}(t)$ are calculated by Eq. (4) and Eq. (5), respectively.

$$
\begin{aligned}
& \lambda_{i}(t)=\lambda_{i}(t-1)+\delta_{C D} \times\left[1-5 / 2 \times\left|O_{i}(t-1)-B_{i}(t-1)\right|+1 / 2 \times\left[O_{i}(t-1)-B_{i}(t-1)\right]^{2}\right] / 2 \\
& P_{i}(t)=P_{i}(t-1)+\gamma_{C D} \times\left[B_{i}(t-1)-P_{i}(t-1)\right] / 2
\end{aligned}
$$

The values of $\delta \in[0,1]$ and $\gamma \in[0,1]$ indicate the speed with which cognitive dissonance operates.

If the homophily theory is applied, $\lambda_{i}(t)$ and $P_{i}(t)$ are updated by Eq. (6) and Eq. (7), respectively.

$$
\begin{aligned}
& \lambda_{i}(t)=\lambda_{i}(t-1)\left(1-\delta_{H}\right)+\bar{\lambda}_{i}(t-1) \times \delta_{H} \\
& P_{i}(t)=P_{i}(t-1)\left(1-\gamma_{H}\right)+\bar{P}_{i}(t-1) \times \gamma_{H}
\end{aligned}
$$

To ensure that $\lambda_{i}(t) \in[0,1]$ and that $P_{i}(t) \in[-1,1]$, the values are truncated as follows:

$$
\begin{aligned}
& \lambda_{i}(t)= \begin{cases}+1 & \text { if } \lambda_{\mathrm{i}}(\mathrm{t})>1 \\
\lambda_{\mathrm{i}}(\mathrm{t}) & \text { if } 0 \leq \lambda_{\mathrm{i}}(\mathrm{t}) \leq 1 \\
0 & \text { if } \lambda_{\mathrm{i}}(\mathrm{t})<0\end{cases} \\
& P_{i}(t)= \begin{cases}+1 & \text { if } \quad \mathrm{P}_{\mathrm{i}}(\mathrm{t})>1 \\
\mathrm{P}_{\mathrm{i}}(\mathrm{t}) & \text { if }-1 \leq \mathrm{P}_{\mathrm{i}}(\mathrm{t}) \leq 1 \\
-1 & \text { if } \mathrm{P}_{\mathrm{i}}(\mathrm{t})<-1\end{cases}
\end{aligned}
$$

In summary, the ORMAC simulation calculates the value of $B_{i}(t)\left(B_{i}(t) \in\{-1,0,1\}\right)$ for each citizen agent $i$ that allows her to maximize the utility function described in Eq. (3) at time t. 


\section{Design of the ORMAC Agent}

The simulation uses only one type of agent, the citizen agent. Each agent has both geographical attributes and mandatory attributes required by the social theories. These attributes are described below. The techniques used to build the synthetic population were described in Chapter 1: Evaluation of Data Availability and Worldwide Survey of Data Quality.

\section{Geographical Attributes}

The first step in building the synthetic population was geospatially locating the 31 million agents within grids approximately one kilometer on each side using the LandScan Global techniques for disaggregation. At the end of this step, the following attributes were assigned to each agent: district_id, cell_id, agent_id, x_coordinate, and y_coordinate. The $\mathrm{x}$ and $\mathrm{y}$ coordinates of an agent were the same as the coordinates of the center of the cell to which the agent belonged. The 31 million agents were distributed as shown in Figure 1-3 (in Chapter 1).

An agent's ideology (P) is determined from the Gallup World Poll, which reports the percentage of individuals, by three ethnic group designations, that are progovernment, pro-Taliban, and neutral. Among Pashtuns, these ideological affiliations vary depending on whether the agent resides in the war zone. Among Tajiks and all other ethnic groups, ideological affiliations do not vary geographically.

Table 5-2. Mindshare from Gallup World Poll

\begin{tabular}{|l|c|c|c|c|}
\hline & $\begin{array}{c}\text { Pashtun- } \\
\text { War Zone }\end{array}$ & $\begin{array}{c}\text { Pashtun- } \\
\text { Not War Zone }\end{array}$ & Tajik & Other Ethnicity \\
\hline Pro-Government & 53 & 70 & 71 & 95 \\
\hline Neutral & 33 & 16 & 27 & 4 \\
\hline Pro-Taliban & 14 & 14 & 2 & 1 \\
\hline
\end{tabular}

Attitudes seem to have significant cleavages along ethnic (Pashtun, Tajik, and other), location (north, south), and combat proximity (war zone vs. nonwar zone) lines. A map of war zone location is reproduced below. The order of the leader $(\mathrm{O})$ in the district is set to 1 if the district is in the nonwar zone, and the order is set to 0 if the district is in the war zone because we believe that leaders in the nonwar zone are more likely to be pro-government, while leaders in the war zone are under pressure from the Taliban and are more likely to take a neutral stand. The violence factor (V) is derived from the map below and the Gallup Poll. In the war zone, $32 \%$ of poll participants said that they felt safe, while $68 \%$ of those in the nonwar zone felt safe. Therefore, $\mathrm{V}=0.68$ for agents in the nonwar zone, and $\mathrm{V}=0.32$ for agents in the war zone. 
The indicator database for how economic welfare is perceived by the individual and is tied to behaviors in support of the leader in the Afghan case is highly dependent upon the social atom's proximity to opium production. The Afghan example is simpler than most countries where corruption indices might create usable indicator databases where economic activity under leadership control may be more complex. However, in Afghanistan, the primary source of discretionary economic resources that a leader might use to obtain behavior according to his order is directly connected to the leader's ability to control opium profits or his ability to control law enforcement actions. A map of opium districts is shown in Figure 1-6 (in Chapter 1).

The coercion motive is captured by $\alpha$, the relative magnitude of the resources that can be brought by the leader against an agent. We have hypothesized that agents who belong to the majority ethnic group in the district will have more resources brought against them than those in minority ethnic groups. Within each district, each ethnic group is ranked in one of three categories, from the most numerous to the least. If an agent belongs to the most numerous ethnic group, the resources $(\alpha)$ that may be brought against the agent are quantified by an $\alpha$ value of 0.75. Agents in the second-ranked ethnic group are given an $\alpha$ value of 0.5 , and agents in the least numerous ethnic groups are given a value of 0.25 .

We assumed that agents' initial perceptions $(\lambda)$ of the leader's legitimacy follow a normal distribution with a mean of 0.5 and a standard deviation of 0.25 .

\section{Mandatory Agent Attributes}

To calculate the utility function in Eq. (3), the following attributes must be quantified for each agent: $\mathrm{O}, \alpha, \mathrm{P}, \mathrm{E}, \mathrm{V}$, and $\lambda$. P and $\lambda$ change over time according to the learning theory applied. The rest of the attributes remain constant for the entire duration of the simulation.

In summary, each ORMAC ACSES agent is represented by a tuple (district_id, cell_id, ethnicity_id, agent_id, x_coordinate, y_coordinate, $O, \alpha, P_{i}(t), \mathrm{E}, \mathrm{V}$, $\left.\lambda_{i}(t)\right)$. The combination of "district_id + cell_id + ethnicity_id + agent_id" uniquely identifies an agent. 


\section{Flow diagram of the ORMAC Simulation}

The flow diagrams in Figures 5-4 and 5-5 illustrate the computational logic of the ORMAC ACSES simulation.

Figure 5-4. Flow diagram of "Social Mobilization Theories + Cognitive Dissonance Theory"

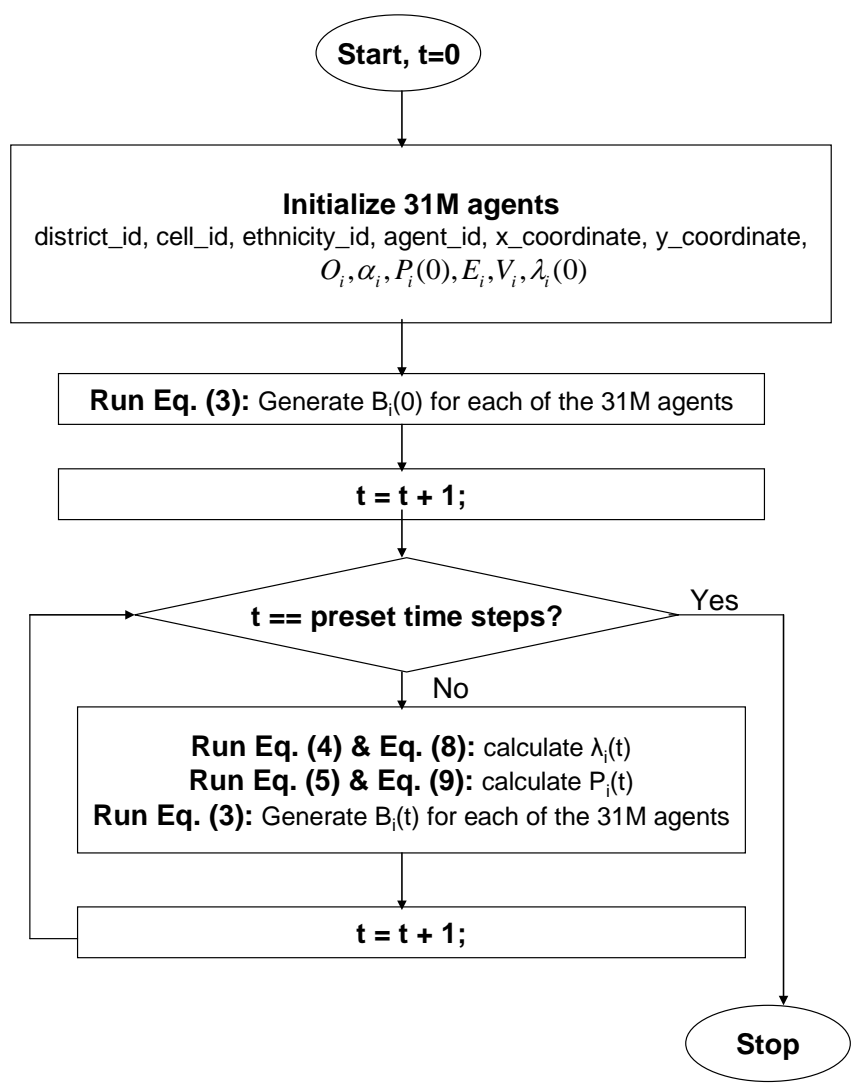


Figure 5-5. Flow diagram of "Social Mobilization Theories + Homophily Theory"

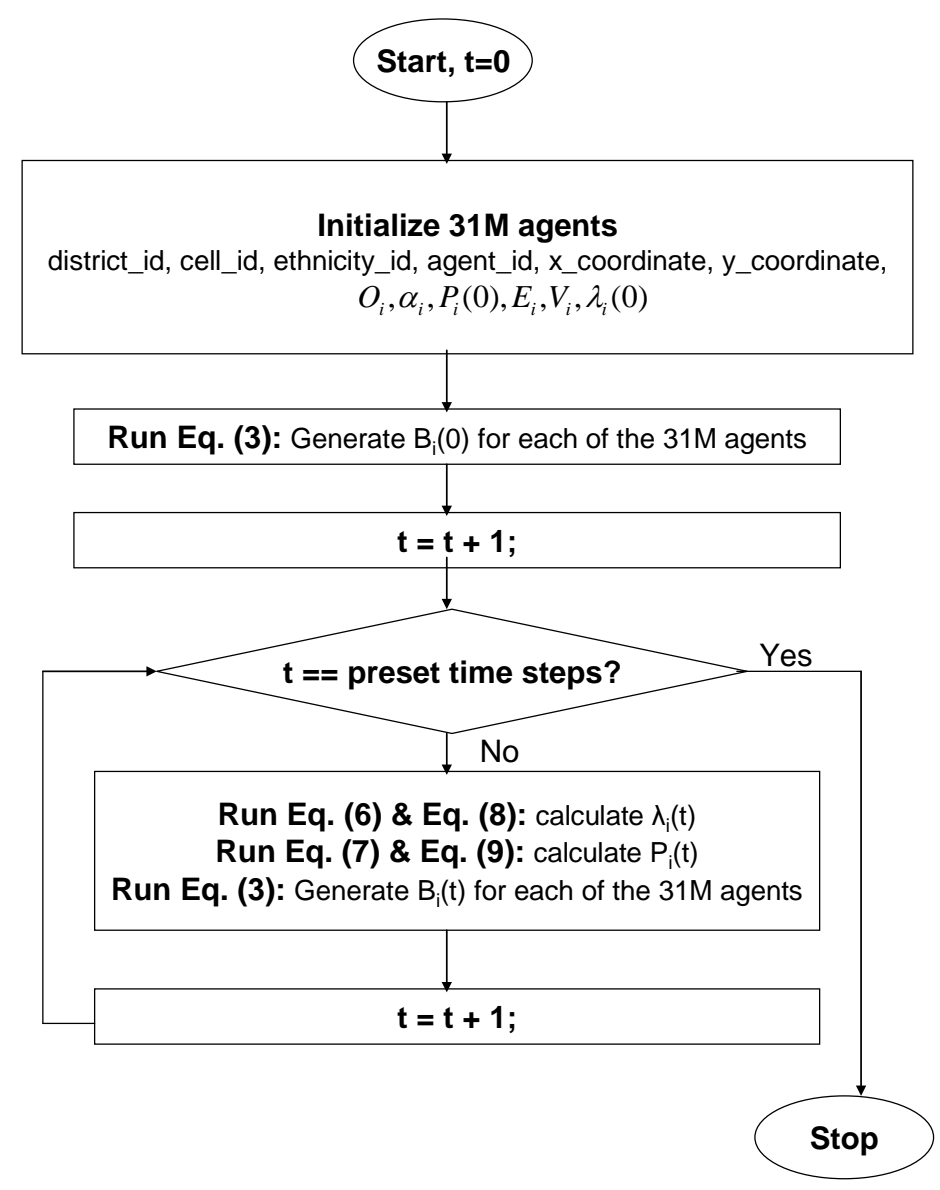

\section{ORMAC Simulation Output Description - Feasibility Experiment}

We operated the ORMAC simulation to compare the distributions of Afghan terrorist incidents in 2006 against the detailed results of the ORMAC simulation system. This experiment was not intended as a demonstration of predictability but rather as evidence that disaggregated systems can generate sufficiently detailed results to allow comparison with real-world data.

After operating the ORMAC simulation, we were able to observe small district-bydistrict differences in the rise of pro-Taliban populations. If we assign district identification numbers from 1 to 329, we can plot the distribution of these pro-Taliban populations and compare these predicted pro-Taliban cells with the geographic distribution of Afghan terror incidents. If data on the district-by-district distribution of these pro-Taliban populations can be correlated with increased terrorist incidences, then we might be able to evaluate courses of remedial action by leadership theory. The plot of these minority (pro-Taliban) population distributions is shown in Figure 5-6. 
Figure 5-6. Percentage pro-Taliban minority opinion by district

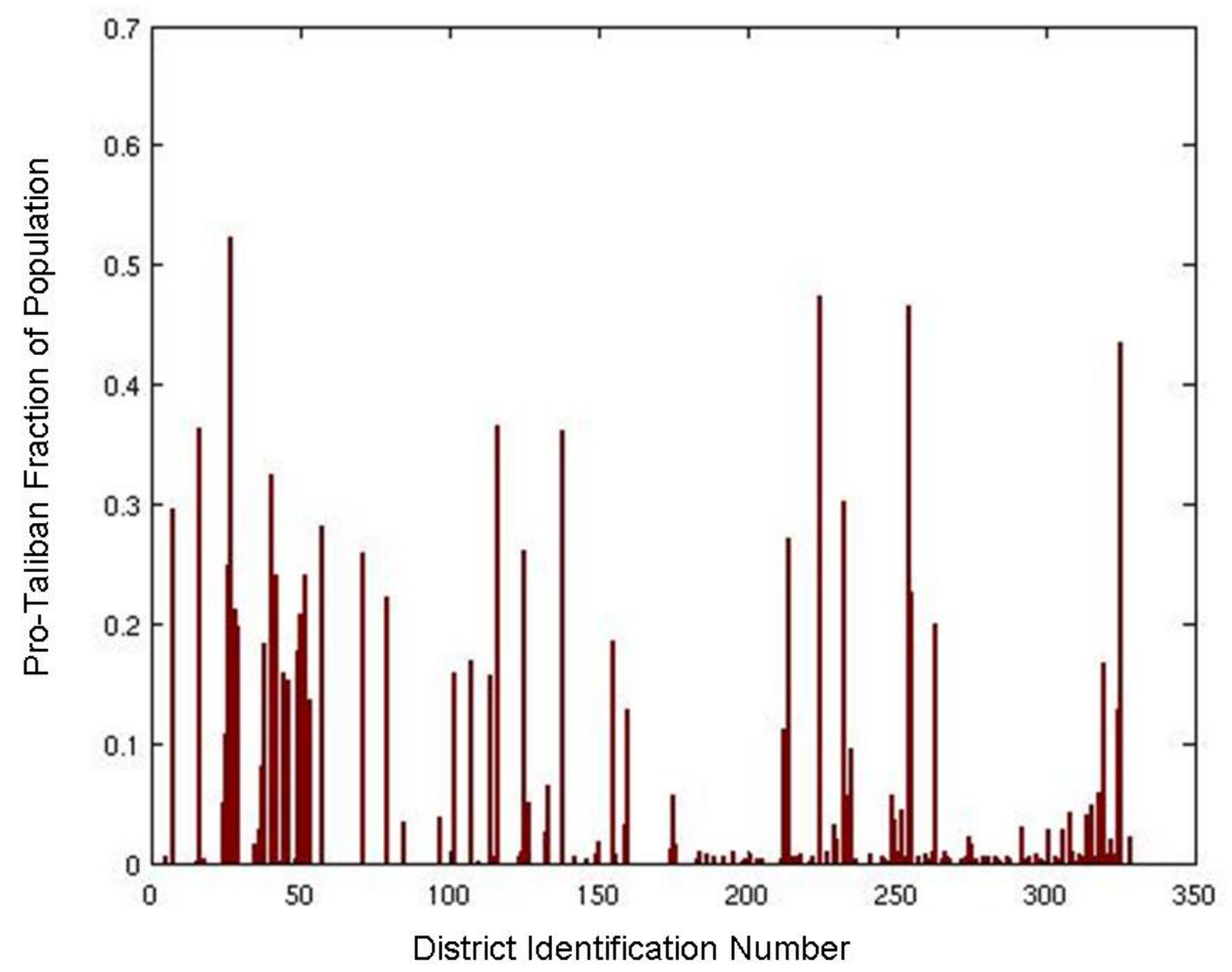

A geographic representation of the distributions (Figure 5-7) shows that the pro-Taliban populations seem to cluster around the boundaries between the Pashtun-dominated areas and those areas dominated by other ethnic groups.

Figure 5-7. Distribution of pro-Taliban minority opinion

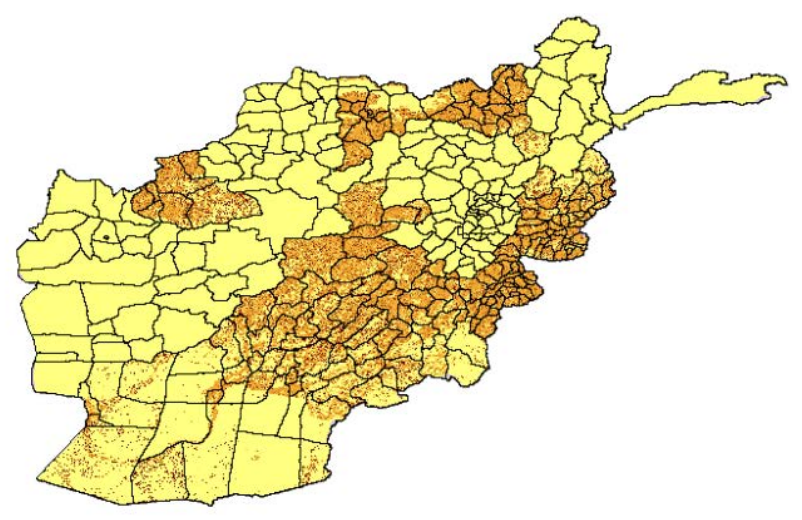


Figure 5-8 maps the distribution of terrorist incidents according to the National Center for Counterterrorism, which maintains the Worldwide Incident Tracking System (WITS).

Figure 5-8. Distribution of Taliban terrorist incidents

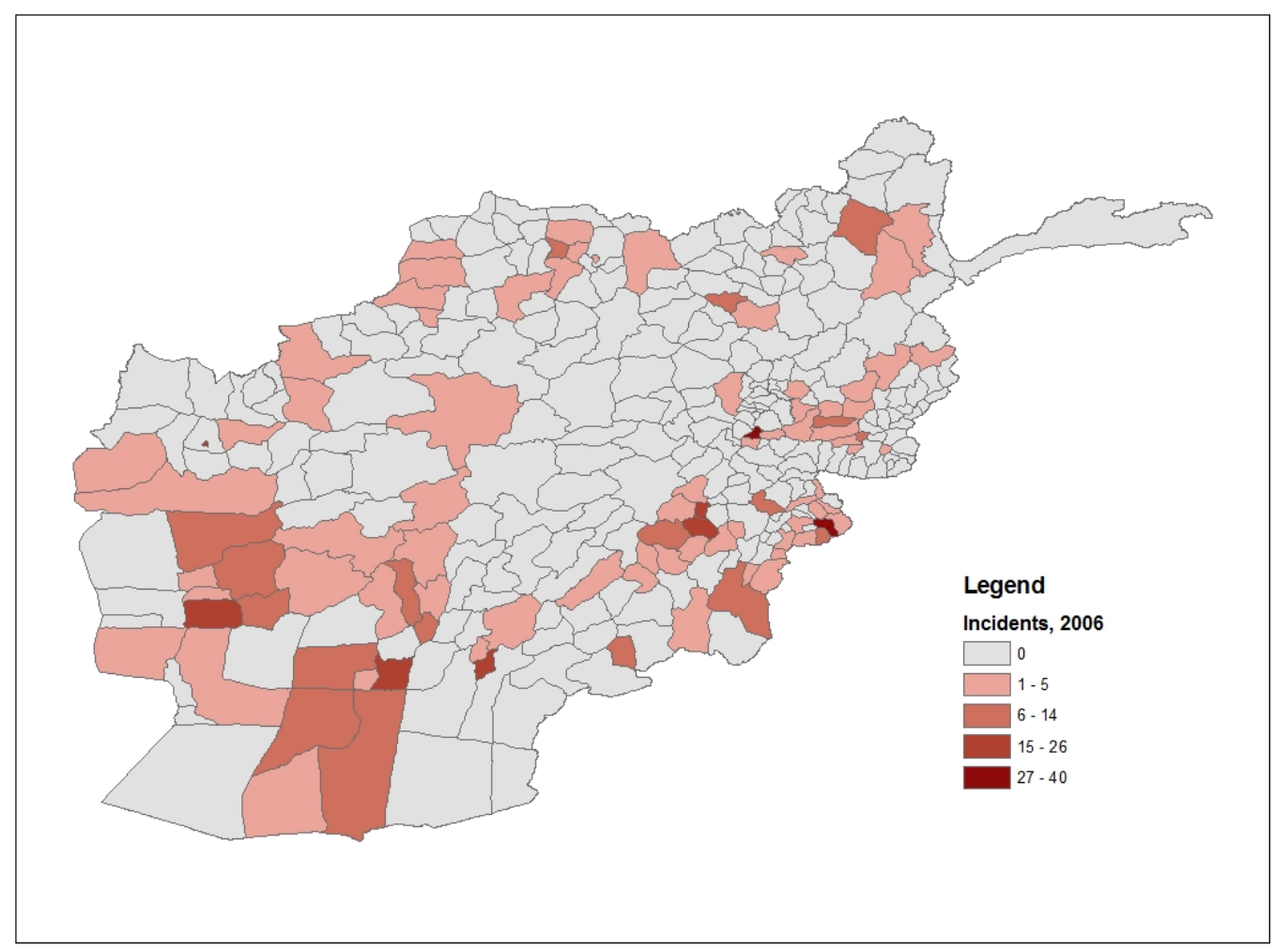

If we examine a small subregion (Ghazni Province) of the plotted terror incidents (Figure 5-9) and compare it to the district population of Taliban supporters (Figure 5-10) calculated in the ORMAC system, the qualitative correlation is apparent. 
Figure 5-9. Distribution of Taliban terror incidents with Ghazni province highlighted

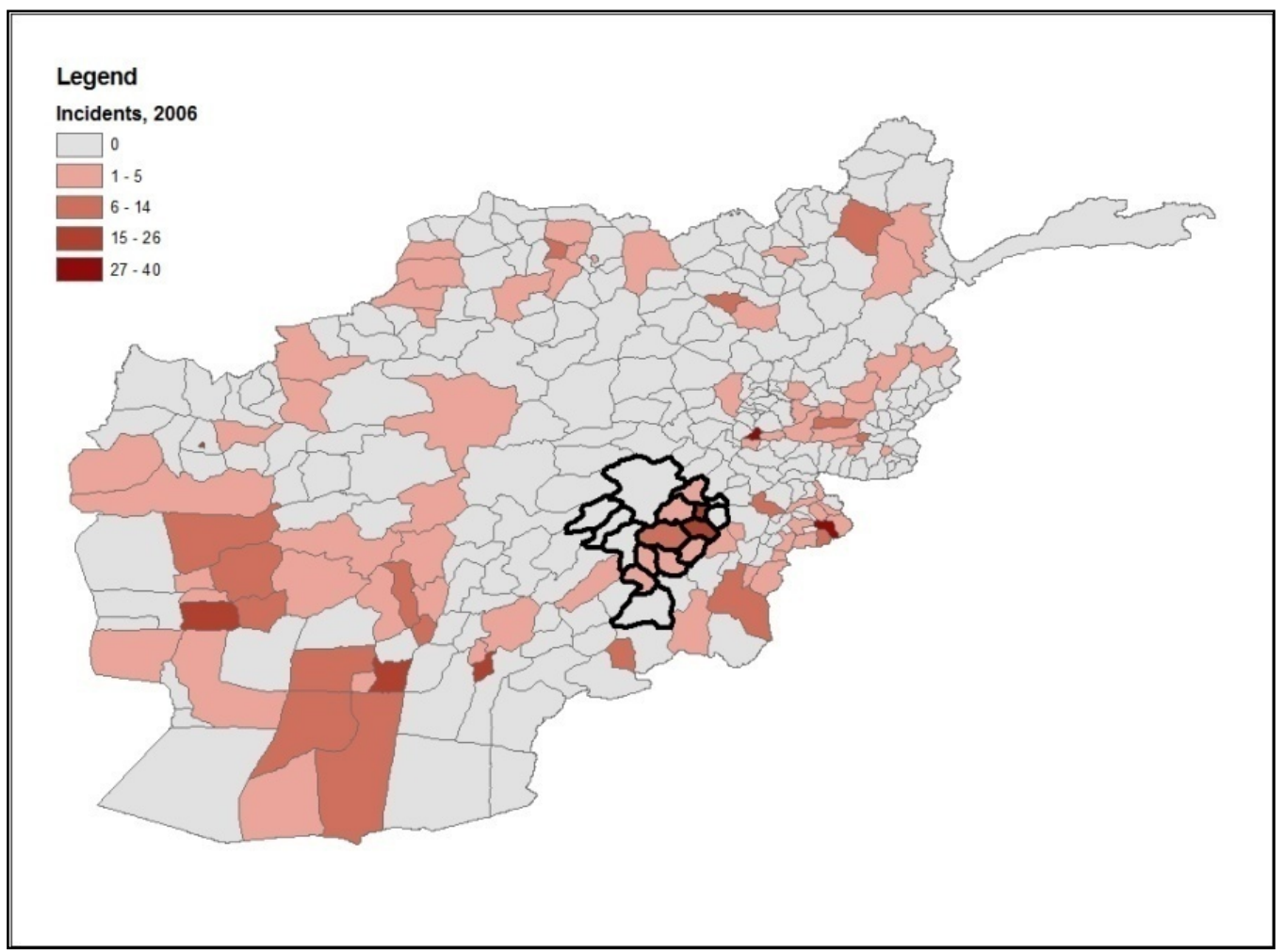

Figure 5-10. Distribution of pro-Taliban minority opinion under representative theory

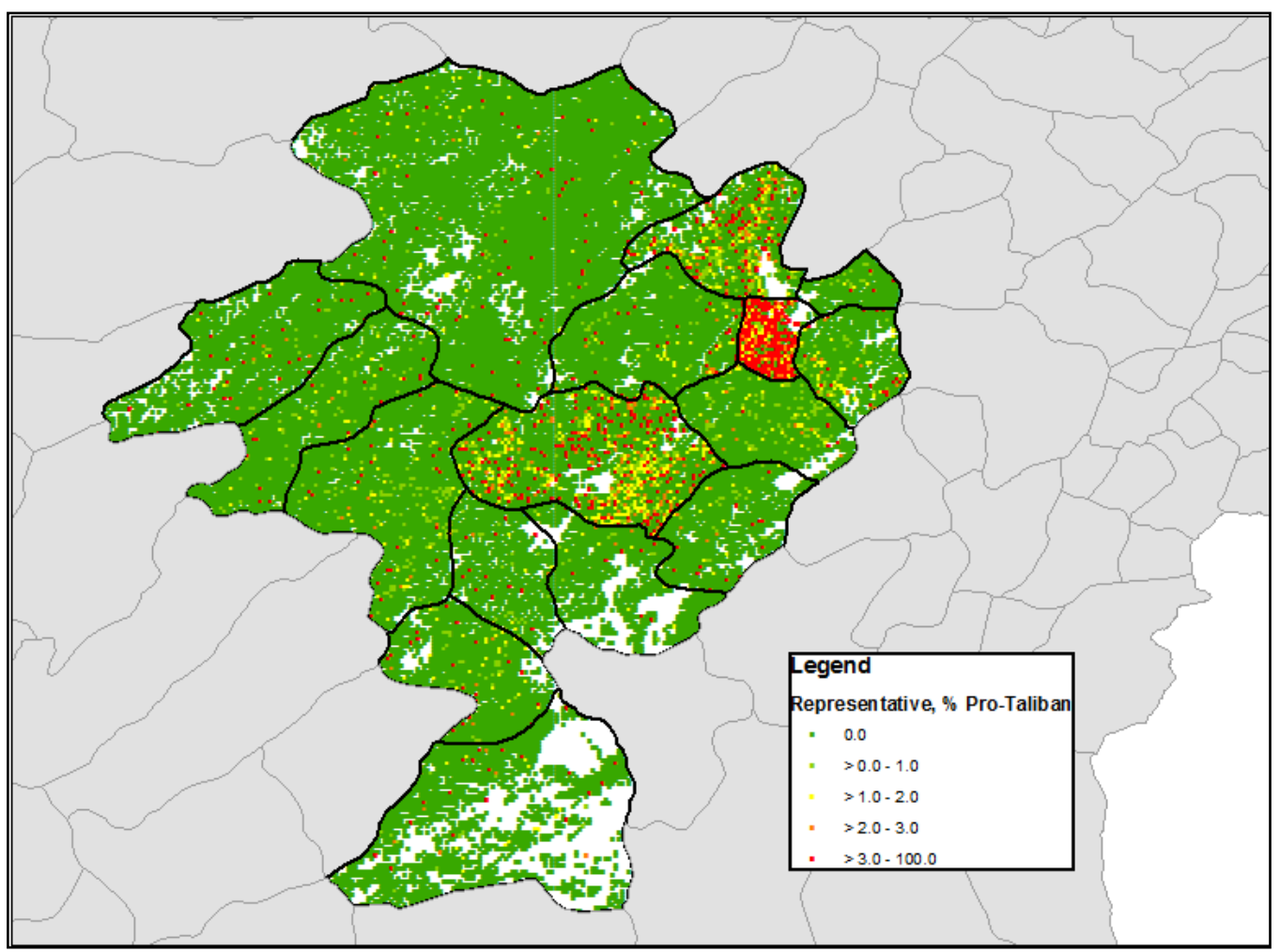


If we plot the location of terrorist incidents against the location of pro-Taliban populations, the resulting histogram (Figure 5-11) resembles the type of histogram we would predict under a coercive leadership.

Figure 5-11. Minority pro-Taliban opinion correlated with 2006 terrorist incidents

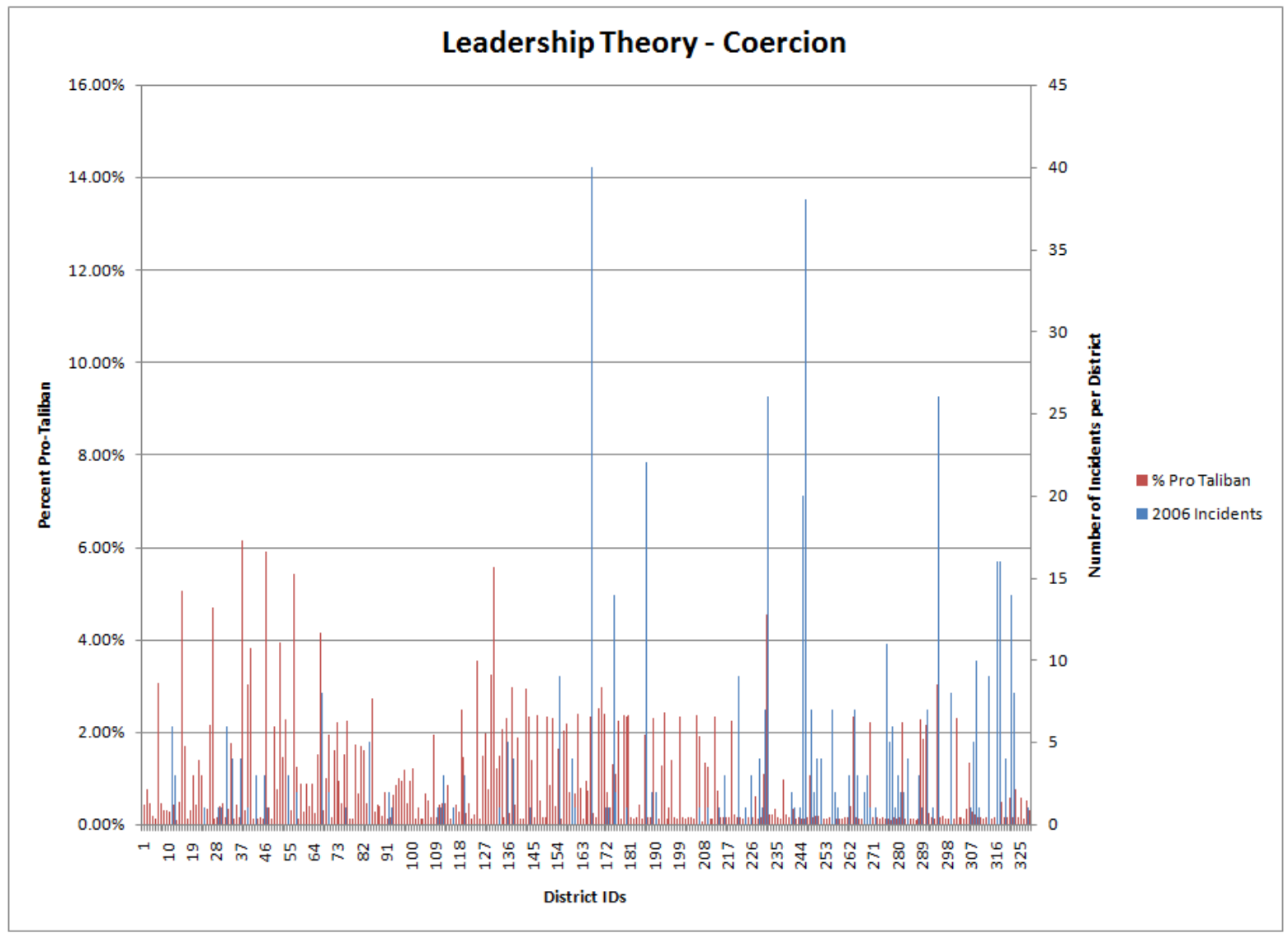

The correlation coefficients for three different leadership theories ${ }^{4}$ are shown in the table below. Coercion theory produces the strongest correlation with the data. Thus, manipulation of the input variables for the coercion model may allow the commander to see the impact of different courses of action intended to quell terror incidents in specific locations.

\footnotetext{
${ }^{4}$ The three leadership theories are defined as follows: (1) coercion: people of the local population are motivated primarily by coercion from their leader, which depends on his resources, (2) legitimacy: people of the local population are motivated primarily by their high loyalty to their leader, and (3) representative: people of the local population are motivated primarily by their economic situation, their dislike of violence, and their ideology.
} 
Table 5-3. Correlation coefficients between minority opinion and WITS databases

\begin{tabular}{|l|l|}
\hline Variables & Pearson Correlation Coefficients \\
& Reference: 2006 Incidents \\
\hline Coercion, \% Pro-Taliban & $.49564(<0.0001)$ \\
\hline Legitimacy, \% Pro-Taliban & $.20776(<0.0001)$ \\
\hline Representative, \% Pro-Taliban & $.48307(<0.0001)$ \\
\hline Incidents, 2007 & $.83159(<0.0001)$ \\
\hline
\end{tabular}

From this exercise, a few insights into the ORMAC system can be identified.

1) Although the coercion and representative theories have similar correlations to the actual NCTC data, the ORMAC simulation system identified coercion theory as most representative of the ground truth observed in Afghanistan.

2) Resolution of data allows small subpopulations to be identified. These small populations were not apparent in the more aggregated NetLogo model but were apparent at higher resolution modeling. Testing the modeling results against the presence of these populations would be a good validation exercise for this modeling approach.

3) Higher numbers of agents reveal features important to course-of-action analysis. We will discuss this further in the next section.

4) Using real-world data, it is possible to select among social science models and tease out proximate root causes. Because variables important in coercion theory produce the best correlation with real-world data, courses of action predicted by coercion theory to suppress problematic populations may be the most promising.

5) Model calibration approaches may be developed to illustrate forward anticipation as a next step. Calibration of the model with more historical data sets might lead to more refined weighting functions that would improve the correlations and forecasting skill.

Following this comparison of the ORMAC system to actual 2006 data, a comparison experiment between the NetLogo and ORMAC systems was conducted. The objective of this experiment was to observe whether different results were obtained when the scale of the simulation was disaggregated from 5000 agents to 31 million agents. 


\section{Comparison of NetLogo and ORMAC Results in Response to the Commander's Questions}

To compare the two simulation systems, we selected the following top-level, hypothetical question:

Taliban terrorist activity has increased in Ghazni Province in 2007 over the levels of 2006. My objective is to drastically reduce the level of violence in 2008. If I have the following courses of action at my disposal-

- surging of troops into Taliban-controlled areas,

- providing additional economic and social development resources to local leaders,

- replacing local leaders with more supportive officials,

- reducing the number of Taliban entering the border regions, and

- implementing social, political, or economic actions to reduce general population support for the Taliban

- what would the impact of these courses of action be?

A subset of the question above was selected to perform the side-by-side comparison. The complete NetLogo response for the commander's question can be found in Chapter 2 .

Selection of the experimental run to compare the effects of different levels of aggregation between the NetLogo system and the ORMAC system is described below.

Although the ORMAC simulation is capable of simulating the movement of fighter agents and the geographical movement of violence, for the comparison we selected a special case in which soldier agents were neither initially present nor "regenerated". This decision was made because the NetLogo scale for the citizen agents is different from the soldier agents in a variable and nondeterministic way. Therefore, the special case of no troops allows direct, side-by-side comparison of the different simulations. Following are the initializing conditions:

- No soldier agents were simulated. In the NetLogo simulation, "Add Agent at Setup", "Regeneration", and "Surge" were set to zero.

- "Influence" and "Repression" were both turned off.

- Initial O, P, R, E, and V values were determined as described below. For this simulation, our goal was to create input variables as similar to University of North Carolina at Charlotte's (UNCC’s) input variables as possible.

O - Values of Leader's Order are dependent upon ethnicity as follows (max of 1, min of $1)$ :

Pashtun $(\mathrm{O}=0.0)$

Tajik $(\mathrm{O}=0)$

Hazara $(\mathrm{O}=1)$

Uzbek $(\mathrm{O}=0)$ 
Turkmen $(\mathrm{O}=-1)$

Balouch $(\mathrm{O}=1)$

Nuristan $(\mathrm{O}=1)$

P - Values of political ideology are dependent on ethnicity. The mean value (with a standard deviation of 0.25 ) for all agents of each ethnicity is given below (max of 1 , min of -1$)$ :

Pashtun $(\mathrm{P}=-0.1)$

Tajik $(\mathrm{P}=0.45)$

Hazara $(\mathrm{P}=0.8)$

Uzbek $(\mathrm{P}=0)$

Turkmen $(\mathrm{P}=0.3)$

Balouch $(\mathrm{P}=-0.25)$

Nuristan $(P=-0.75)$

$\mathrm{R}$ - Resources leaders may use against agents:

$\mathrm{R}=1$ for all agents

E - Economic welfare of agents varied depending on the presence of opium in a district. In districts where opium was present, $38 \%$ of agents had $E=0.8718$; otherwise $\mathrm{E}=0$. The data used to determine the presence or absence of opium in a district was a patch-level map of opium districts used by UNCC. By comparing the geographical distribution of patches with opium with the location of districts in Afghanistan, six districts were determined to be opium producers (districts 27713, 27721, 27731, 27734, 27744, and 27747).

V- Violence welfare was not updated.

In the Netlogo formulation, violence is given as $\mathrm{vc}^{-} \mathrm{vc}^{-}$, where $\mathrm{vc}$ is defined as "foes"/ ("friends" + "foes"), and $\mathrm{vc}^{-}$is the worst possible value of vc. In this simulation, $\mathrm{V}$ reflects the perceived level of violence that individuals reported in the Gallup World Poll survey. $\mathrm{V}=0.69$ for all agents.

The Ghazni region as defined in the NetLogo simulation encompasses the following districts in the ORMAC simulation: 27701, 27713, 27721, 27723, 27726, 27731, 27734, 27736, 27744, 27746, 27747, 27761, 27764, 27767, 27778, 27785.

The learning theories were turned off.

We varied two parameters: number of days per tick $(3,7,14)$ and leadership theory $(\mathrm{C}, \mathrm{L}$, $\mathrm{R})$. C represents coercion theory, $\mathrm{R}$ corresponds to representative theory, and $\mathrm{L}$ represents legitimacy theory. The runs, given in the format of days per tick_theory, are

run1: 3L

run2: 3R

run3: $3 \mathrm{C}$

run4: 7L 
run5: 7R

run6: 7C

run7: $14 \mathrm{~L}$

run8: $14 \mathrm{R}$

run9: $14 \mathrm{C}$

Each leadership theory assigns the following values to the seven weights of the utility function:
C: $0.1 \quad 0.5$
$0.1 \quad 0.1$
0.2
0.0
0.0
R: $0.05 \quad 0.05 \quad 0.3$
0.3
$0.3 \quad 0.0$
0.0
L: 0.6
0.10 .1
0.2
$0.0 \quad 0.0$
0.0

Events were defined from the WITS data as district-level assassination attempts within Ghazni province. At each event the order of the leader changed. The number of days per tick determines the total number of ticks simulated and determines at which tick each event occurs:

Table 5-4. Points of event insertion

\begin{tabular}{|l|c|c|c|c|c|c|}
\hline & $\begin{array}{c}\text { Event - } \\
1\end{array}$ & Event - 2 & Event - 3 & Event - 4 & Event - 5 & End \\
\hline 3days/tick & 11 & 26 & 27 & 47 & 58 & 68 \\
\hline 7days/tick & 5 & 12 & 12 & 21 & 25 & 35 \\
\hline 14days/tick & 3 & 6 & 6 & 13 & 16 & 26 \\
\hline
\end{tabular}

After each event, the $\mathrm{O}$ values for Pashtuns decrease by 0.2 . The $\mathrm{O}$ value is truncated to be within the range of $[-1.0,1.0]$.

The results of these comparison experiments are plotted in Appendix 10 - Visual Plots of NetLogo and ORMAC Comparisons. At the completion of the nine experiments, the percentages of pro-Taliban, neutral, and pro-government agents were estimated and recorded. Because the stochastic elements randomized the initial conditions in the NetLogo model, we constrained the starting point for pro-Taliban and neutral agents to be time step zero and allowed the pro-government agents to constitute the residual. Comparisons such as these may help improve model outputs, in this case to correct bias errors. Simple correlation coefficients between the NetLogo and ORMAC simulation systems were calculated for each run, and the population's mindshare as a function of time was also plotted. Differences in the results of the two simulation systems could then be ascribed to differences in scale.

One interesting aspect is that the ORMAC outputs appear more responsive to disruptive events when there is a response, while the Netlogo outputs exhibit more random fluctuations but less response to the events. The cause of this observation is not precisely known, but the random fluctuations may be due to stochastic elements embedded within 
the NetLogo code. The lack of responsiveness is a common feature when acute events are averaged over large populations such as is necessary in aggregated systems.

In situations where both NetLogo and ORMAC exhibit what appear to be "legitimate" (i.e., occurring at the expected time steps) responses to events, the NetLogo responses seem somewhat damped compared to the ORMAC responses. This indeed may be caused by resolution effects, even though this empirical "rule" has a few exceptions. The lack of any ORMAC response (even the changes in NetLogo appear to be no more than random fluctuations) to the disruptive events when the representativeness (R) theory is instantiated may be worth noting. The lack of a leader may have less immediate effect on followers when the predominant behavior is representative. However such social explanations must be exercised with care given that the simulation runs appear pretty flat in each case when this theory is implemented (runs 2, 5, and 8). This may suggest an artifact of the specific experimental design. Although the causal explanations offered here are plausible but not proven unless further simulations are performed, the value of simple metrics (e.g., bias and first differences) together with visual representations may be apparent from the discussions here.

Traditional statistical distance measures are shown next. The correlation coefficients (CC) in the table below further indicate that the increase in the number of days per tick does not significantly improve the dependency between systems with respect to the number of agents in each class.

Table 5-5. Correlation coefficients between NetLogo and ORMAC outputs

\begin{tabular}{|c|c|c|c|c|c|}
\hline Run & Days/tick & Theory & Pro-Taliban & Neutral & Pro-Govt \\
\hline 1 & 3 & L & 0.9750 & 0.8870 & -0.2867 \\
\hline 4 & 7 & L & 0.9776 & 0.9463 & -0.4136 \\
\hline 7 & 14 & L & 0.9610 & 0.9109 & -0.6806 \\
\hline 2 & 3 & R & -0.2505 & -0.2614 & 0.1203 \\
\hline 5 & 7 & R & -0.3966 & -0.4101 & 0.1839 \\
\hline 8 & 14 & R & $-\mathbf{0 . 5 6 0 1}$ & $-\mathbf{0 . 6 0 4 8}$ & 0.2603 \\
\hline 3 & 3 & C & 0.8479 & 0.1196 & -0.2215 \\
\hline 6 & 7 & C & 0.9361 & 0.4923 & -0.3166 \\
\hline 9 & 14 & C & 0.9479 & 0.5612 & -0.4139 \\
\hline
\end{tabular}

If the pro-government behavior is ignored (because the ORMAC variations in that category are limited), the correlations are strongest for legitimacy (L) theory, followed by coercion (C). Correlations for representativeness (R) are negative, confirming the intuition that resolution effects may be very important for that theory. The mean squared errors (MSE) are rather low (Table 5-6), suggesting that on average for all the simulation time steps, the errors are not too high. 
Table 5-6. MSE between NetLogo and ORMAC outputs

\begin{tabular}{|c|c|c|c|c|c|}
\hline Run & Days/tick & Theory & Pro-Taliban & Neutral & Pro-Govt \\
\hline 1 & 3 & L & 0.0013 & $\mathbf{0 . 1 8 0 4}$ & $\mathbf{0 . 1 6 7 7}$ \\
\hline 2 & 3 & R & $\mathbf{0 . 0 6 4 4}$ & $\mathbf{0 . 1 6 4 8}$ & $\mathbf{0 . 0 2 4 4}$ \\
\hline 3 & 3 & C & $\mathbf{0 . 0 3 3 3}$ & $\mathbf{0 . 3 1 3 2}$ & $\mathbf{0 . 1 4 6 7}$ \\
\hline 4 & 7 & L & $\mathbf{0 . 0 0 1 8}$ & $\mathbf{0 . 1 5 6 7}$ & $\mathbf{0 . 1 7 1 5}$ \\
\hline 5 & 7 & R & $\mathbf{0 . 0 6 0 2}$ & $\mathbf{0 . 1 6 9 7}$ & $\mathbf{0 . 0 2 9 6}$ \\
\hline 6 & 7 & C & $\mathbf{0 . 0 2 8 4}$ & $\mathbf{0 . 3 0 8 9}$ & $\mathbf{0 . 1 5 3 2}$ \\
\hline 7 & 14 & L & $\mathbf{0 . 0 0 3 3}$ & $\mathbf{0 . 1 5 2 1}$ & $\mathbf{0 . 1 6 9 1}$ \\
\hline 8 & 14 & R & $\mathbf{0 . 0 5 9 8}$ & $\mathbf{0 . 1 5 9 6}$ & $\mathbf{0 . 0 2 6 3}$ \\
\hline 9 & 14 & C & $\mathbf{0 . 0 2 9 4}$ & $\mathbf{0 . 3 1 5 9}$ & $\mathbf{0 . 1 5 5 0}$ \\
\hline
\end{tabular}

A pair-wise comparison of the correlations between ORMAC and NetLogo runs based on instantiations of different leadership theories is shown in Tables 5-7 and 5-8. The results are interesting not just for exploring how the outputs from multiple theories relate to each other, but also for seeing how the relations themselves change between the two implementations.

Table 5-7. Correlation coefficients between runs for NetLogo outputs

\begin{tabular}{|c|c|c|c|c|c|}
\hline Runs & Days/tick & Theories & Pro-Taliban & Neutral & Pro-Govt \\
\hline 1,2 & 3 & $\mathbf{L}, \mathbf{R}$ & 0.7942 & 0.4615 & 0.6081 \\
\hline 1,3 & 3 & $\mathbf{L}, \mathbf{C}$ & 0.9166 & 0.4490 & 0.9096 \\
\hline 2,3 & 3 & $\mathrm{R}, \mathrm{C}$ & 0.5329 & -0.4681 & 0.7442 \\
\hline 4,5 & 7 & $\mathbf{L}, \mathbf{R}$ & 0.7759 & 0.3778 & 0.5842 \\
\hline 4,6 & 7 & $\mathbf{L}, \mathrm{C}$ & 0.9149 & 0.5631 & 0.9198 \\
\hline 5,6 & 7 & $\mathrm{R}, \mathrm{C}$ & 0.5144 & -0.4346 & 0.7077 \\
\hline 7,8 & 14 & $\mathbf{L}, \mathbf{R}$ & 0.8190 & 0.4502 & 0.6347 \\
\hline 7,9 & 14 & $\mathbf{L}, \mathbf{C}$ & 0.9378 & 0.6350 & 0.9006 \\
\hline 8,9 & 14 & $\mathrm{R}, \mathrm{C}$ & 0.6023 & -0.2874 & 0.7761 \\
\hline
\end{tabular}

Table 5-8. Correlation coefficients between runs for ORMAC outputs

\begin{tabular}{|c|c|c|c|c|c|}
\hline Runs & Days/tick & Theories & Pro-Taliban & Neutral & Pro-Govt \\
\hline $\mathbf{1 , 2}$ & $\mathbf{3}$ & L,R & $-\mathbf{0 . 1 8 7 9}$ & $-\mathbf{0 . 1 8 7 9}$ & -1.0000 \\
\hline $\mathbf{1 , 3}$ & $\mathbf{3}$ & L,C & $\mathbf{0 . 9 9 9 6}$ & $\mathbf{0 . 9 9 9 6}$ & $\mathbf{1 . 0 0 0 0}$ \\
\hline 2,3 & 3 & R,C & $-\mathbf{0 . 1 8 3 3}$ & $-\mathbf{0 . 1 8 3 4}$ & -1.0000 \\
\hline 4,5 & 7 & L,R & $-\mathbf{0 . 2 5 5 1}$ & $-\mathbf{0 . 2 5 5 1}$ & -1.0000 \\
\hline $\mathbf{4 , 6}$ & $\mathbf{7}$ & L,C & $\mathbf{0 . 9 9 9 6}$ & $\mathbf{0 . 9 9 9 6}$ & $\mathbf{1 . 0 0 0 0}$ \\
\hline 5,6 & 7 & R,C & $-\mathbf{0 . 2 5 0 3}$ & $-\mathbf{0 . 2 5 0 5}$ & -1.0000 \\
\hline 7,8 & 14 & L,R & -0.3626 & -0.3627 & -1.0000 \\
\hline 7,9 & 14 & L,C & $\mathbf{0 . 9 9 9 8}$ & $\mathbf{0 . 9 9 9 8}$ & $\mathbf{1 . 0 0 0 0}$ \\
\hline 8,9 & 14 & R,C & -0.3533 & -0.3534 & -1.0000 \\
\hline
\end{tabular}


The correlation between the outputs from the legitimacy $(\mathrm{L})$ and coercion $(\mathrm{C})$ theories, as implemented here, is high and appear to be least affected by the simulation granularity (i.e., NetLogo versus ORMAC). However, the relations appear completely altered for any comparison involving the representativeness (R) theory. Once again, this may be an artifact of the specific simulation, as all the plots with this theory appear relatively flat [Appendix 10, Figures A10-1 (b), (e), and (h)]. One other observation is that the change in number of days per tick seems to cause no statistical difference in the dependency between runs with respect to the number of agents in each class.

A detailed comparison of a relatively "lumped" or low-resolution model (e.g., the NetLogo-based implementation) with a relatively more spatially "distributed" or highresolution model (e.g., the ORMAC-based implementation) typically entails one of two approaches: either aggregate the distributed model outputs to the scales of the lumped model and compare at the aggregate scales or allocate the lumped model outputs to the scales of the distributed model and compare at the higher resolutions. A comparison based on aggregation may be fairer because the allocation process is not necessarily welldefined and may introduce errors. (In contrast, aggregation processes are typically welldefined; for example when agent counts are considered, a simple sum would almost always be considered appropriate.). Indeed, most of the previous comparisons in this section would fall in that category. However, in situations where the end users or stakeholders demand higher resolution outputs, or when the underlying dominant social processes can be best captured at higher resolutions, a comparison at those resolutions may be more appropriate. In such cases, allocations become necessary for comparisons. In the absence of ancillary information, simple methods like equal or area-weighted allocations are probably the only options.

In our case, the aggregate "patch" level outputs generated from NetLogo need to be allocated to the finer grids at which data are obtained from the Geographical Information Systems (GIS) and which are ultimately used by the ORMAC-based simulations. The simulation results must be compared at scales that matter to decision-makers (e.g., district levels in Afghanistan).

Motivated by the points discussed above, and driven by the need to develop proof-ofconcept comparison metrics and methods, we uniformly allocated the aggregate NetLogo outputs to the resolutions of the ORMAC simulations. Specifically, the number of agents for each class and each patch was converted into the corresponding number of agents for each district by multiplying the uniformly distributed number of agents by the number of patches that equal the geographical size of each district. In a sense, this is just an areaweighted allocation strategy. We focused the comparison on "Run 1" (see Table 5-6) and a few districts for illustrative purposes. 
Table 5-9. Linear correlation between ORMAC and NetLogo runs at district levels

\begin{tabular}{|c|c|c|c|c|}
\hline RUN & $\begin{array}{c}\text { District } \\
\text { ID }\end{array}$ & Pro-Taliban & Neutral & Pro-Govt \\
\hline \multirow{3}{*}{1} & 27713 & 0.9744 & 0.8855 & 0.0000 \\
\cline { 2 - 5 } & 27723 & 0.9759 & 0.8890 & 0.0000 \\
\cline { 2 - 5 } & 27726 & 0.9759 & 0.8897 & 0.0000 \\
\cline { 2 - 5 } & 27731 & 0.9744 & 0.8856 & -0.2867 \\
\cline { 2 - 5 } & 27747 & 0.9740 & 0.8846 & 0.0000 \\
\hline
\end{tabular}

Table 5-9 shows high correlations between the ORMAC and NetLogo outputs for proTaliban and neutral agents. The zero or negative correlations for pro-government agents may be ignored given that the number of these agents remains relatively constant during the simulation time period. The high correlation at a district level indicates that a simple allocation was able to handle the resolution effect in terms of the correlation measure. In addition, we note that correlation is one measure that captures linear associations among data fluctuations, but more nuanced differences may not be obvious by looking at this measure alone. Thus, the effects of resolution are clearly seen in the district-level agent distributions over time.

The following summarizes the insight gained from these comparison experiments:

1. The aggregated approach yields different results when stochastic elements are substituted for ground truth about initial conditions.

2. This randomness causes the aggregated system to be less responsive to acute localized events such as assassinations.

3. The two systems stabilized at similar, but not identical, final results for mindshare at the end of the simulation time.

The ORMAC and NetLogo simulation results differ in additional ways that would lead to different evaluations about potential courses of action. Under each of the theories, the NetLogo system produced final results significantly different from both the ORMAC system and the WITS data used for validation. To ensure that the level of aggregation does not adversely impact the usefulness of these simulations, disaggregated models executed on fast platforms within flexible and robust simulation environments may be important to capture the sudden, significant destabilizing events within such a simulation environment.

Until very recently, computational frameworks and widely available hardware have limited the selection of approaches to highly aggregated systems (the first two approaches). New architectures can now manage $10^{8}$ agents and allow simulations with predictive value in computationally tractable times. These architectures provide to the social theorist the entire simulation space to select the most efficient system based solely upon the theory and population under study.

We have learned a number of lessons: 
1. It is possible to manipulate 31 million agents and perform a calculation to compare different leadership theories without judging whether other theories are more appropriate to the question at hand.

2. Behaviors observed in disaggregated models differ from those observed in aggregated models. The former can be compared to real-world events.

3. Correlation with significant events is highly dependent on the scale used for the simulation. Depending on the theory to be examined, fast granular models are likely to be needed.

Future work is needed to define

1) the computational space, architecture, and framework required to apply many of the most common social theories;

2) the trade-off between increased simulation speed, agent aggregation, and observance of emerging or actionable results;

3) the criteria and metrics that might be used to select the appropriate computational scheme - desktop, cluster, cloud, or HPC applications-for a given region, population, question, and sequence of theories to be instantiated.

\section{Insights and Conclusions}

The objective of Task 5 was to illustrate the feasibility of operating a complex agentbased simulation system that could generate courses of action in computational times much less than 24 hours. The approach to this task included

- estimating the maximum hypothetical complexity of the agents that provides for each dimension of the HSBC, incorporating elements of modern social theory;

- characterizing the maximum complexity of the environment that allows for realtime updating of the environment with unfolding events;

- developing literature examples of analogous systems of comparable complexity;

- comparing employed computational resources to those currently available with similar run times for case studies.

Testing the 31-million-agent model of Afghanistan's population demonstrated the ability to computationally control and analytically manipulate a system with the large number of agents (i.e., $10^{8}$ ) necessary to model regions at the individual level. Simulations in computationally tractable times can be performed with computational costs that range from less than a dollar for simulations with simple interactions to a few hundred dollars for the largest and most complex simulations.

If the linear scaling holds, a 31-million-agent simulation would require approximately 5.2 hours to complete a time step. A 1000-time-step simulation would exceed the computational time permitted at 5200 hours. However, multiple processor systems can reduce the computational time to a practical range at modest cost. 
Even at the extreme end of computational intensity, these costs are well within the costs of similar simulation programs currently under development. Between these two extremes is a rich three-dimensional trade space that trades speed of simulation, simulation costs, and observed emergent behavior.

This effort compared and contrasted two different theory-driven, agent-based modeling approaches and explored how each could be implemented and evaluated. A very highresolution model and a more aggregated model were implemented using the same synthetic population and the same base set of social theories to explore a variety of response actions to an increase in Taliban-inspired terrorist activities. Simulations with agents representing individual social atoms were able to predict the development of small populations of pro-Taliban members that were geographically correlated with the terrorist incidents as recorded in the Worldwide Incident Tracking System. These correlations were very different at aggregation scales on the level of 5000 to 1.

The lesson learned from this task is that compromises taken in the past to aggregate populations because small platforms were employed to perform social simulations can be eased through HPC applications. These applications expand the opportunity to leverage human terrain results within a simulation environment and will offer new options for strategic planners.

The resulting insight is that HSBC simulations are an HPC problem in the same sense that weather prediction is an HPC problem; the computations can be done in hours or days (not months, like some "big physics" problems), but the need to meet an "actionable" deadline imposes a completion time of minutes or hours and, consequently, a need for HPC. The cost and availability of these resources have now placed these resources within the reach of the combatant commands. 


\section{References}

\section{Chapter 1}

Bhaduri, B. L., E. A. Bright, P. R. Coleman, and J. Dobson. 2002. LandScan: Locating people is what matters. Geoinformatics 5(2):34-7.

Bhaduri, B. L., E. A. Bright, P. R. Coleman, and M. L. Urban. 2007. LandScan USA: A high-resolution geospatial and temporal modeling approach for population distribution and dynamics. GeoJournal 69:103-17.

Dobson, J. E., E. A. Bright, P. R. Coleman, R. C. Durfee, and B. A. Worley. 2000. A global population database for estimating populations at risk. Photogrammetric Engineering \& Remote Sensing 66(7).

Ferguson, N. M., D. A. T. Cummings, S. Cauchemez, C. Fraser, S. Riley, A. Meeyai, S. Iamsirithaworn, and D. S. Burke. 2005. Strategies for containing an emerging influenza pandemic in southeast Asia. Nature 437:210, 213. http://www.nature.com/nature/journal/v437/n7056/pdf/nature04017.pdf

Gregory, I. A place in history: A guide to using GIS in historical research. AHDS Guides to Good Practice, ISSN 1463-5194. May, 2002.

\section{Chapter 2}

Ajzen, I. 2001. Nature and operation of attitudes. Annual Review of Psychology 52:2758.

Berger, J., C. L. Ridgeway, M. H. Fisek, and R. Z. Norman. 1998. The legitimation and delegitimation of power and prestige orders. American Sociological Review 63:379405.

Bourdieu, P. 1984. Distinction: A social critique of the judgement of taste. Cambridge, Massachusetts: Harvard University Press.

Carley, K. 1986. An approach for relating social structure to cognitive structure. Journal of Mathematical Sociology 12:137-89.

Coleman, J. S. 1990. Foundations of social theory. Cambridge, Massachusetts: Harvard University Press.

Festinger, L. 1957. A theory of cognitive dissonance. Stanford, California: Stanford University Press.

Nisbett, R. E., and L. Ross. 1980. Human inference: Strategies and shortcomings of social judgment. Englewood Cliffs, New Jersey: Prentice-Hall. 
Olson, J. M., P. A. Vernon, J. A. Harris, and K. Jang. 2001. The heritability of attitudes: A study of twins. Journal of Personality and Social Psychology 80:845-60.

Opp, K.-D., and C. Gern. 1993. Dissident groups, personal networks, and spontaneous cooperation: The East German Revolution of 1989. American Sociological Review 58:659-80.

Opp, K.-D., and W. Roehl. 1990. Repression, micromobilization, and political protest. Social Forces 69:521-48.

Petty, R. E., T. T. Wegener, and L. R. Fabrigar. 1997. Attitudes and attitude change. Annual Review of Psychology 48:609-47.

Tversky, A., and D. Kahneman. 1981. The framing of decisions and the psychology of choice. Science 211:453-8.

\section{Chapter 3}

Bond, D., J. Bond, C. Oh, J. Jenkins, and C. Taylor. 2003. Integrated data for events analysis (IDEA): An event typology for automated events data development. $J$ Peace Res 40(6):733.

Carlsson, C., and O. Hagsand. 1993. DIVE --- A multi-user virtual reality system. In 1993 IEEE Virtual Reality Annual Symposium (VRAIS '93), Seattle, Oregon, September 1993.

Chandy, K. M., and J. Misra. 1978. A nontrivial example of concurrent processing: distributed simulation. Proceedings of COMPSAC, pp. 822-6.

Chandy, K. M., and J. Misra. 1979. Distributed simulation: A case study in design and verification of distributed programs. IEEE Transactions on Software Engineering, SE-5, 5, 440-52.

Darema, F. 2004. Dynamic data driven applications systems: A new paradigm for application simulations and measurements. Volume 3038/2004 of Lecture Notes in Computer Science. Springer Berlin: Heidelberg.

Fujimoto, R. M. 1990. Parallel discrete event simulation. Communications of the ACM 33(10):30-53.

Funkhouser, T. A. 1995. RING: A client server system for multi-user virtual environments. ACM SIGGRAPH Special Issue on 1995 Symposium on Interactive 3D Graphics, Monterey, California, pp. 85-92.

Gintis, H. 2007. The dynamics of general equilibrium. The Economic Journal 117(523):1280-309. 
Kennedy, C., and G. Theodoropoulos. 2005. Towards intelligent data-driven simulation for policy decision support in the social sciences. Technical Report CSR-05-9, University of Birmingham, School of Computer Science. Available online at: ftp://ftp.cs.bham.ac.uk/pub/tech-reports/2005/CSR-05-9.ps.gz.

Lamport, L. 1978. Time, clocks, and the ordering of events in a distributed system. Communications of the ACM 21(7):558-65.

Macedonia, M. R., M. J. Zyda, D. Pratt, P. T. Barham, and S. Zeswitz. 1994. NPSNET: A network software architecture for large scale virtual environments. Presence 3(4):265-87.

Macedonia, M. R., and M. J. Zyda. 1997. A taxonomy for networked virtual environments. IEEE Multimedia 4(1):48-56.

National Research Council. 2006. Defense modeling, simulation, and analysis: Meeting the challenge. National Academies Press.

Oren, T. I., S. K. Numrich, A. M. Uhrmacher, L. F. Wilson, and E. Gelenbe. 2000. Agentdirected simulation-Challenges to meet defense and civilian requirements. Simulation Conference Proceedings 2:1757-62.

Plale, B., D. Gannon, D. Reed, S. Graves, K. Droegemeier, B. Wilhelmson, and M. Ramamurthy. 2005. Towards dynamically adaptive weather analysis and forecasting in LEAD. ICCS workshop on Dynamic Data Driven Applications.

Schrodt, P. 2001. Automated coding of international event data using sparse parsing techniques. International Studies Association, Chicago, February, 2001.

Singh, G., L. Serra, W. Png, and H. Ng. 1994. BrickNet: A software toolkit for networkbased virtual worlds. Presence 3(1):19-34.

Stytz, M. R.1996. Distributed virtual environments. IEEE Computer Graphics and Applications 16(3):19-31.

Testa, J., M. Aldinger, K. Wilson, and C. Caruana. 2006. Achieving standardized livevirtual constructive test and training interactions via TENA. Paper No. 2900 at The Interservice/Industry Training, Simulation \& Education Conference (I/ITSEC).

Wilensky, U. 1999. NetLogo. Center for Connected Learning and Computer-Based Modeling, Northwestern University, Evanston, Illinois. http://ccl.northwestern.edu/netlogo/.

\section{Chapter 4}

Eisenhardt, K. M. 1989. Building theories from case study research. The Academy of Management Review 14(4):532-50. 
Golden-Biddle, K., and K. Locke. 1993. Appealing work: An investigation of how ethnographic texts convince. Organization Science 4(4):595-616.

Jick, T. D. 1979. Mixing qualitative and quantitative methods: Triangulation in action. Administrative Science Quarterly 24(4):602-11.

Kittredge, R. L., and A. Polguère. 2000. The generation of reports from databases. In The Handbook of Natural Language Processing, by Hermann Moisl, H. L. Somers Robert Dale, Chapter 11. CRC Press.

Kukich, K. 1983. Design of a knowledge-based report generator. Proceedings of the 21st annual meeting on Association for Computational Linguistics, pp 145-50.

Kumar, M., D. Das, and A. I. Rudnicky. 2007. Summarizing non-textual events with 'briefing' focus. Proceedings of RIAO 2007, Pittsburgh, Pennsylvania.

Maybury, M. T. 1995. Generating summaries from event data. International Journal of Information Processing and Management: Special Issue on Text Summarization 31(5):735-51.

Miles, M. B. 1979. Qualitative data as an attractive nuisance: The problem of analysis. Administrative Science Quarterly 24:590-601.

Pentland, B. T. 1995. Grammatical models of organizational processes. Organization Science 6(5):541-56.

Pentland, B. T. 1999. Building process theory with narrative: From description to explanation. The Academy of Management Review 24(4):711-24.

Reiter, E., S. Sripada, J. Hunter, J. Yu, and I. Davy. 2005. Choosing words in computergenerated weather forecasts. Artificial Intelligence 167(1-2):137-69.

Sabherwal, R., and D. Robey.1993. An empirical taxonomy of implementation processes based on sequences of events in information system development. Organization Science 4(4): 548-76.

Van Maanen, J. 1979. Reclaiming qualitative methods for organizational research: A preface. Administrative Science Quarterly 24(4):520-6.

Yu, J., E. Reiter, J. Hunter, and C. Mellish. 2006. Choosing the content of textual summaries of large time-series data sets. Natural Language Engineering 13(1):2549.

\section{Chapter 5}

Germann, T. C., K. Kadau, I. M. Longini, and C. A. Macken. 2006. Mitigation strategies for pandemic influenza in the United States. PNAS 103(15):5935-40. 
Barrett, C., S. Eubank, V. A. Kumar, and M. Marathe. 2004. Understanding large scale social and infrastructure networks: A simulation based approach. In SIAM News, March 2004.

Barrett, C., J. P. Smith, and S. Eubank. 2005. Modern epidemiology modeling. Scientific American, March 2005.

Eubank, S., H. Guclu, S. Kumar, M. Marathe, A, Srinavasan, Z. Toroczkai, and N. Wang. 2004. Modeling disease outbreaks in realistic urban social networks. Nature 429:180-4.

Munson, Z. 2001. Islamic mobilization: Social movement theory and the Egyptian Muslim brotherhood. Sociological Quarterly 42(4):487-510.

Benford, R., and D. Snow. 2000. Framing processes and social movements: An overview and assessment. Annual Review of Sociology 26:11-39.

Earl, J., A. Martin, S. A. Soule, and J. D. McCarthy. 2004. The use of newspaper data in the study of collective action. Annual Review of Sociology 30:65-80.

Finkel, S. E., and J. B. Rule. 1986. Relative deprivation and related psychological theories of civil violence: A critical review. In K. and G. Lang, Research in Social Movements, Conflicts and Change 9:47-69. Greenwich, Connecticut: JAI Press.

Giugni, M. 1998. Was it worth the effort? The outcomes and consequences of social movements. Annual Review of Sociology 24:371-93.

Gurney, J. N., and K. J. Tierney. 1982. Relative deprivation and social movements: A critical look at twenty years of theory and research. Sociological Quarterly 23:3347.

Jenkins, C. 1983. Resource mobilization theory and the study of social movements. Annual Review of Sociology 9:527-53.

Meyer, D. S. 2004. Protest and political opportunities. Annual Review of Sociology 30:125-98.

Picardo, N. 1997. New social movements: A critical review. Annual Review of Sociology 23:411-30.

Snow, D. A., and R. Machalek. 1984. The sociology of conversion. Annual Review of Sociology 10:167-90.

Strang, D., and S. A. Soule. 1998. Diffusion in organizations and social movements: From hybrid corn to poison pills. Annual Review of Sociology 24:265-90.

Useem, B. 1998. Breakdown theories of collective action. Annual Review of Sociology 24:215-38. 
Chandler, J. 2005. The explanatory value of social movement theory. Strategic Insights $4(5)$.

Silverman, B. G., G. Bharathy, B. Nye, and R. J. Eidelson. 2007. Modeling factions for effects based operations: Part I-Leaders and followers. Comput Math Organ Theory DOI 10.1007/s10588-007-9017-8.

Modi, P. J., M. Spiros, W. M. Mongan, W. Regli, and I. Mayk. 2006. Towards a reference model for agent based systems. AAMAS, Hakodate, Hokkaido, Japan. 


\section{Appendix 1 - Research Plan}

\section{Objectives of the Program}

The goal of this project is to develop a TiVo ${ }^{\mathrm{TM}}$-like simulation capability to monitor, assess, forecast and respond to adverse human behavioral developments around the world in near real-time. This seedling proposal identifies some of the key technical challenges in developing such a system and proposes a research plan for assessing the feasibility of overcoming them.

The current generation of societal modeling and simulation uses existing architectures that are based on linking independently developed models to provide some measure of predictability of HSBC effects resulting from specific diplomatic, information, military, and economic (DIME) triggering events. The current generation of modeling and simulation systems is characterized by the following attributes:

- These are modular systems, with each module (model) optimizing one or two elements of the HSBC domain. Multiple models are then linked together to cover the HSBC spectrum. The degree of aggregation in the models does not permit sensitivity analysis with respect to the causes of surprising changes in human behavioral dynamics without prohibitive computational power or analytical time.

- Current agent-based models allow the agents to interact within their environment without constraints. The models generate a large number of potential futures but fail to quantify the probability of any single stream of future events. They incorporate little or no modern social scientific theory or analysis to adjust the rule base to mimic real-world observations. To generate realistic behaviors consistent with observations, the entire range of HSBC theories must be leveraged to inform the simulation rule sets.

- These systems employ a flexible architecture to combine models from a variety of constituencies (such as the NATO application), thereby permitting a community (M\&S community, alliance community, interagency coordination and coalitions) to jointly experiment and understand intracommunity interactions. However, current models and simulations provide no capability for real-time or near realtime data updates as surprising events unfold or rapid changes occur in societal dynamics.

- These systems cannot conduct a sensitivity study of key causal effects of an observed change in a region's stability in reasonable computing times. They provide only limited capability for analysts to select streams of future events important for the question at hand. Current systems are difficult for analysts to intuitively comprehend and easily control in an agile manner.

Many recent advances permit us to consider the next generation of modeling and simulation systems that can support the ability to anticipate adverse human behavioral developments and that can evaluate alternative courses of action to mitigate the impact of these developments on U.S. national security interests. They include

- new approaches to multi-agent systems that allow us to envision agents with goals and that allow events that the goal-oriented agent could respond to according to the rule sets that span the HSBC domain; 
- advances in high-performance computing (HPC) resources that can assist computationally intensive cases when large numbers of agents are required to adequately describe regional stability and when aggregation complicates sensitivity analyses;

- emergence of architectures that can handle $10^{8}$ agents, and that facilitate disaggregation to the level of individuals behaving according to modern social theories within community scales important to regional stability.

This research thrust has as its central goal the ability to simulate human behavior in a way that anticipates adverse future developments and supports alternative course of action assessments to mitigate them. The objective of the proposed research is to develop the next generation of social simulations that integrates advances in social science theory and computational sciences. This new generation of societal simulation will be characterized by the following attributes:

- These systems will be independent of the linkage of separate models (i.e., a political model linked to an economic model, linked to an infrastructure model, etc.) that are limited by aggregation and by unrealistic behavior rule sets.

- The new systems will be able to conduct sensitivity studies of key causal effects of an observed change in a region's stability (surprises) in reasonable computing times.

- These systems will allow analysts to intuitively comprehend and control the simulation of future event streams and will facilitate analysis of historical precursors.

Key to the next generation of social modeling and simulation is the ability to (a) accurately simulate observed surprise events that threaten or impact a region's stability, (b) model observed historical events that led to the event with sufficient transparency to allow insight into causal factors, and (c) use this insight to evaluate DIME options for influencing emerging events that will increase regional stability.

\section{Research Plan}

The first phase addresses the feasibility of the five scientific tasks that are cornerstones of the new simulation system:

1. Identify and characterize global data sources and granularity needed to synthesize and model populations at the level required to support the simulation environment. The metrics for this task are as follows:

- Demonstrate, or document the feasibility of, direct use of observed or simulated data to seed the behavioral dynamics governing heterogeneous agents representing the entire HSBC domain at each scale of aggregation.

- Demonstrate or document the adequacy of available public domain or classified data sources that relate directly to individual human behavior as well as overall social or economic phenomena, with the objective of guiding 
the formulation of CAS-based models capable of generating actionable predictive insights as well as for modeling surprising emergent behavior.

- Demonstrate or document the adequacy of disparate, multiscale, and possibly dynamic data sources that can validate the causal patterns and the predictive insights extracted from CAS-based models for social or economic systems.

- Demonstrate the feasibility of utilizing methodologies and tools that can employ multiscale, multisource, disparate, dynamic, and sparse and/or possibly incomplete observations to continually improve the simulation models.

- Demonstrate the feasibility of utilizing the observed and simulated data in a scalable fashion within a realistic modeling and simulation environment. Specifically, demonstrate the feasibility of enabling the online data-model feedback loop in an environment with more than a 100 million agents, with complex behavior based on realistic social or economic theory, and with disparate data feeds coming in at real-time or near real-time.

In summary, for the seedling effort, the overall metric for Task 1 is the degree of success in providing convincing evidence that the data and related tools exist for enabling the rest of the tasks (2-5).

2. Identify, compare, and contrast several different theory-driven, agent-based modeling approaches, and explore how each could be implemented and evaluated. The task will begin by exploring three candidate approaches:

- static, hard-coded rules;

- dynamic, fitness function-driven, evolvable rules that are initialized from state-of-the-art sociological models of human behavior;

- dynamic, evolvable rules that are initialized from random models of human behavior.

The metric for this task will be the model's response time and accuracy. Of those that are sufficiently responsive, only the CAS models that achieve $80 \%$ predictive accuracy will be considered adequate or successful.

3. Prototype the capabilities needed to update the agent environment with real-time data, and train the agents so that their emergent behavior will mimic real-world events without prescribing the eventual result. Following is the metric for this effort:

- Demonstrate, or document the existence of, a scheme that maps real-time data updates to a semantic model understood by the CAS used in these experiments. 
- Demonstrate, or document the existence of, a CAS agent design that can incorporate real-time data updates.

4. Demonstrate the ability to account for the context precipitating a surprising or emergent historical event, and characterize causal factors followed by the development of future doctrinal actions to forecast future events. The metrics for assessing the feasibility of this task are as follows:

- Demonstrate, or document the existence of, a CAS design that is sufficiently flexible and true-to-life that it can generate unexpected results (surprises).

- Demonstrate, or document the existence of, a data aggregation system (ontologies, data mappings, extraction processes) that facilitates the review of simulation history to identify potentially causal factors.

- Demonstrate, or document the existence of, a programmatic way to generate a text-based (human) narrative summarizing key simulation events.

5. Demonstrate the ability to computationally control and analytically manipulate a system with the large number of agents (i.e., $10^{8}$ ) necessary to model regions at the individual level. The metrics for task 5 are listed below:

- Demonstrate, or document the existence of, frameworks of more than 100 million agents with ruleset complexities on the order of that found in Task 1.

- Demonstrate, or document the existence of, the ability of high-performance or grid computing resources to accommodate these frameworks.

- Demonstrate, or document the existence of, the ability to perform scenario simulations in less than one day of computational time.

The individual tasks to accomplish these five objectives are listed below.

\section{Task 1. Data Characterization}

- Identify variables, attributes, etc., of data set needed for initial modeling

- Identify sources. What are available and are they sufficient?

- Determine needed versus available attributes and dimensions

- $\quad$ Characterize data (reduce, extract, etc.) and populate spreadsheet

\section{Task 2. Comparison of Three Approaches}

- Identify and generate the three agent-representation candidates (existing social theory, bootstrapped with social theory, emergent from primitives) 
- Simulate the above

- Evaluate and write up

\section{Task 3. Real-Time Updating}

- Identify existing ways of online text analysis, real-time news feeds, etc., that can be mapped to Goldstein categories

- Document approaches for turning categorical data into a state vector delta

\section{Task 4. Accounting for Context and Surprise}

- Train the agents to produce the event, then retrospectively determine causal relationships

- Perform a partial-order temporal analysis of state space to determine the evolution of the society

- Search the literature and generate a narrative from one of the above

- Perform visual analytics

Task 5. Scale Test

- Survey literature of $10^{8}$ agent scalability

- Demonstrate or analyze a similarly scaled, agent-based simulation.

- Compare the demonstrated environment to the anticipated ABS environment. 


\section{Appendix 2 - Data Sources for Independent Variables}

\section{Political}

National government

Legislative bodies, characteristics, role and power relative to executive

Executive roles (president, vice-president, ministers, etc.) and how selected, degree to which positions are filled by members of particular social groups

Representatives (of executive) throughout country: distribution, resources, degree to which national government controls outlying territory

Descriptors (numbers, resources) for:

Administrative bodies (agencies for tax collection, welfare, regulation, licensing, etc.)

Domestic intelligence agencies including secret police

Voting system for selecting representatives and executive, rate of turnover of individuals in elective office

- Corruption data from World Resources Institute (WRI): environmental governance and institutions:

$\underline{\text { http://earthtrends.wri.org/searchable_db/index.php?theme }=10}$

- Politics and Freedom data from WRI: http://earthtrends.wri.org/searchable_db/index.php?theme $=10$

- Data from CIA World Factbook: https://www.cia.gov/library/publications/the-world-factbook/geos/af.html

- Economist Intelligence Unit Country Reports

- http://en.wikipedia.org/wiki/List_of_legislatures_by_country

- Lijphart Elections Archive (http://dodgson.ucsd.edu/lij/)

- International Institute for Democracy and Electoral Assistance worldwide electoral systems data (http://www.idea.int/esd/world.cfm)

- Trinity College's Electoral systems Web site with its least squares (election result disproportionality) index (http://www.tcd.ie/Political_Science/Staff/Michael.Gallagher/ElSystems/i ndex.php)

Judicial system

Distribution and characteristics of courts

Method by which judges are chosen

Distribution and characteristics of police

Level of government that controls police

Penal system: distribution of jails; number, characteristics, and distribution of incarcerated; control over jails

- Jurist Legal Intelligence World Law Web site (http://jurist.law.pitt.edu/world/)

- International Centre for Prison Studies’ World Prison Population List and Efficiency of Courts measure (http://www.prisonstudies.org/) 
- Library of Congress's legal information for the nations of the world (http://www.loc.gov/law/help/guide/nations.html)

Subnational governments

Type, distribution, depth (how many levels), resources, degree of formal and informal independence from higher levels of government

Rules for suffrage and conducting elections, vote participation, freeness and fairness of elections

- Lijphart Elections Archive (http://dodgson.ucsd.edu/lij/)

- International Institute for Democracy and Electoral Assistance voter turnout data (http://www.idea.int/vt/index.cfm)

Political rights and civil liberties, press freedom, degree to which a political opposition is tolerated and can organize

- Freedom House's political rights, civil liberties, and press freedom indices (http://www.freedomhouse.org/)

- Reporters Without Borders Worldwide Press Freedom Index (http://www.rsf.org/article.php3?id_article=24025)

Degree of security and stability

- CIDCM's Peace and Conflict Instability Ledger (http://www.cidcm.umd.edu/pc/chapter02//)

- Economist Intelligence Unit/Vision of Humanity’s Global Peace Index (http://www.visionofhumanity.com/index.php)

- George Mason University’s Political Instability Task Force Internal Wars and Failures of Governance datasets (http://globalpolicy.gmu.edu/pitf/)

- George Mason University’s State Fragility Index (in book Global Report on Conflict, Governance and State Fragility 2007)

- Foreign Policy-Fund for Peace’s Failed States Index (http://www.fundforpeace.org/web/index.php?option=com_content\&task= view\&id=229\&Itemid=366)

Level of corruption with respect to government and the distribution of where that corruption occurs, transparency of government activities

- Transparency International's Corruption Perceptions Index (http://www.transparency.org/policy_research/surveys_indices/cpi)

- International Budget Project's Open Budget Index (http://www.openbudgetindex.org/)

- International Budget Project’s Latin American Index of Budget Transparency (http://www.fundar.org.mx/fundar/indicelat/pg/2007/docregionales.php)

- Global Integrity’s Integrity Scorecard (http://report.globalintegrity.org/)

\section{Military}

Government:

Different institutions and their functions 
- Peacekeeping operational organizations: United Nations Peacekeeping Operations: http://www.un.org/Depts/dpko/dpko/bnote.htm

- Stockholm International Peace Research Institute's FIRST database (http://first.sipri.org/index.php/)

Numbers, distributions, bases and their distribution, ethnic composition

- Monthly summary data of contributors of military and civilian police personnel from 1995-2007: United Nations Peacekeeping Operations: http://www.un.org/Depts/dpko/dpko/contributors/

- Monthly data of gender statistics in peacekeeping military from 2006-2007: United Nations Peacekeeping Operations: http://www.un.org/Depts/dpko/dpko/contributors/gender/dec07.pdf

- Monthly financing data of United Nations Peacekeeping Operations: http://www.un.org/Depts/dpko/dpko/contributors/financing.html

- Yearly fatalities statistics from 1948-2008: United Nations Peacekeeping Operations: http://www.un.org/Depts/dpko/fatalities/ Activities: protect against foreign attack or domestic threats, pursue terrorists, economic

- Peacekeeping activities including military, police, logistics support, mine action service, medical support, norms of conduct, and contingent owned equipment: United Nations Peacekeeping Operations Agency: http://www.un.org/Depts/dpko/dpko/

- Peace agreements (descriptive data only): UN Peacemaker http://peacemaker.unlb.org/advancedsearch.php

Characteristics of members: why joined, pay, distribution of important membership groups (e.g., ethnic groups), experience, loyalties

Domestic, nongovernment (militias, paramilitary groups, terrorist organizations): Size, location, composition in terms of social groups

Allegiances

Activities: types (protection, bombing, kidnapping, fighting), frequencies, locations

Ties to other organizations:

Organizational, informational, or economic ties

To domestic or international, military or political organizations

- Global Security.org's para-military groups database Foreign:

(http://www.globalsecurity.org/military/world/para/index.html/)

Size, locations, loyalties, activities, ties to other countries, ties to other military groups

Conflicts: size, frequency, location, seasonal concentration, casualties, combatants

- Data from UN Environment programme GEO Data Portal: http://www.grid.unep.ch/data/index.php

- Uppsala Conflict Data Project (http://www.pcr.uu.se/research/UCDP/UCDP toplevel.htm/) 
- George Mason University's Armed Conflict and Intervention project data (http://www.systemicpeace.org/aci.htm)

\section{Economic}

Occupational distribution of population

- Data from international labor organization: http://www.ilo.org/public/english/employment/strat/kilm/

Sector distribution of economic activity

- County statistical information database: World Bank datacenter: http://web.worldbank.org/WBSITE/EXTERNAL/DATASTATISTICS/0, contentMDK:20541648 menuPK:64133152 pagePK:64133150 piPK:64 133175 theSitePK:239419,00.html

- IMF development and statistics data

- Industrial statistics from economic statistics of UN statistics division: http://unstats.un.org/unsd/economic_main.htm

- Agricultural and food data from WRI: http://earthtrends.wri.org/searchable_db/index.php?theme $=8$

Distribution of employment in terms of nature of place of employment (government, government-controlled firm, large firm, small business, selfemployed)

Rate of change of those three distributions

Job growth in comparison to population growth

- International Labor Organization’s labor statistics database (http://laborsta.ilo.org/)

Principal products (agricultural, manufactured): absolute and proportional value, concentration of and location of production, location of markets, profit, growth

- Afghanistan data (and other Asian countries) of population, employment, GDP by industrial origin, structure of output, structure of demand, at constant 2002 factor cost, money and banking, government finance, export and trade, direction of trade, balance of payments, international reserves, external indebtedness: Asian Development Bank: http://www.adb.org/Documents/Books/Key_Indicators/2007/pdf/afg.pdf

- National Account data from UN Economic Statistics: http://unstats.un.org/unsd/economic_main.htm

Largest companies: size, locations, domestic or multinational, ties to other companies, ties to government

- Doing business in Afghanistan 2008 from Doing Business project: http://www.doingbusiness.org/ExploreEconomies/Default.aspx?economyi $\mathrm{d}=2$

- Trade statistics from UN Economics Statistics: http://unstats.un.org/unsd/economic_main.htm

Financial system: characteristics of central bank (is there one? is it autonomous?), links of banks to international firms, distribution throughout country, access of population to loans, capitalization 
- Yearly data of external debt, GDP, GNI, life expectancy, population, population growth, school, surface area in 2005 and 2006: World Bank data center:

http://web.worldbank.org/WBSITE/EXTERNAL/DATASTATISTICS/0, contentMDK:20535285 menuPK:1192694 pagePK:64133150 piPK:641 33175 theSitePK:239419,00.html

- FDI flows 2004: from UN conference on trade and development: http://www.unctad.org/sections/dite_dir/docs/wir04_fs_af_en.pdf

- International debts and governmental expenditure data from WRI: http://earthtrends.wri.org/searchable_db/index.php?theme $=10$

Degree to which economy is integrated with and competitive in global economy

- KOF's Index of Globalization (http://globalization.kof.ethz.ch/)

- ATKearney-Foreign Policy’s Globalization Index (http://www.atkearney.com/main.taf?p=5,4,1,127)

- IMD's World Competitiveness Yearbook (http://www.imd.ch/research/publications/wcy/index.cfm)

- World Economic Forum's Global Competitiveness Index (http://www.weforum.org/en/initiatives/gcp/Global\%20Competitiveness\% 20Report/index.htm)

Degree of economic freedom

- Heritage Foundation/Wall Street Journal's Index of Economic Freedom (http://www.heritage.org/research/features/index/index.cfm)

- Fraser Institute's Economic Freedom of the World Index (http://www.freetheworld.com/reports.html)

Degree of income and wealth inequality (Gini)

- World Income Inequality Database (http://62.237.131.23/wiid/wiid.htm)

Extent of business corruption

- Transparency International's Corruption Perceptions Index (http://www.transparency.org/policy_research/surveys_indices/cpi)

- World Bank’s Worldwide Governance Indicators (http://info.worldbank.org/governance/wgi2007/)

Extent and concentration of poverty and hunger

- United Nation's Human Development Report Human Development Index and Human Poverty Index (http://hdr.undp.org/en/statistics/indices/)

- CIESIN's Global Poverty Data, Food Security Catalog, Small Area Estimates of Poverty and Inequality, and Unsatisfied Basic Needs datasets (http://sedac.ciesin.columbia.edu/povmap/)

\section{Social}

Demographic (perhaps by region): Age distribution, gender distribution, birth rate, infant mortality rate, life expectancy, immigration and emigration rates

- Population, health, nutrition, poverty, gender, World Bank data center: http://web.worldbank.org/WBSITE/EXTERNAL/DATASTATISTICS/0, contentMDK:20535285 menuPK:1192694 pagePK:64133150 piPK:641 33175 theSitePK:239419,00.html 
- Population data from UN statistics division of Demographic and Social Concerns: http://unstats.un.org/unsd/demographic/default.htm

Education: years of education (by region, gender?), type of school (religious, vocational, etc.), proportions with professional and technical (engineering) training

- Yearly data of education in 2006: World Bank data center: http://web.worldbank.org/WBSITE/EXTERNAL/DATASTATISTICS/0, contentMDK:20535285 menuPK:1192694 pagePK:64133150 piPK:641 33175 theSitePK:239419,00.html

- Education data from UN statistics division: http://unstats.un.org/unsd/demographic/default.htm

Health care: doctors/other health care people/facilities per 1000 population, nature of health care system (government, private, mixed, etc.), geographic distribution of health care facilities/personnel

- World Bank’s Millennium Development Goals dataset (http://ddpext.worldbank.org/ext/GMIS/gdmis.do?siteId=2\&menuId=LNAV01HOM E3)

Family: distribution of family types (nuclear, polygynous, single-parent, etc.), distribution of marital statuses (by age)

- Marriage and divorce data from UN statistics division: http://unstats.un.org/unsd/demographic/default.htm

Minority groups: major identity-defining characteristics (e.g., social class, ethnic group, caste, clan, region, language, religion, native-born vs. immigrant), relative size of groups, whether minority groups are concentrated geographically or dispersed, relative circumstances of groups, degree to which minority groups are at risk of harm or disappearance

- University of Maryland's Minorities at Risk dataset (http://www.cidcm.umd.edu/mar/)

Principal social cleavages: dimension(s) of cleavage (as above plus age and sex), relative treatment of groups by government, severity of discrimination based on identity, intensity of cleavage(s)

- UN statistics and indicators on women and men: http://unstats.un.org/unsd/demographic/default.htm

- University of Maryland's Minorities at Risk dataset (http://www.cidcm.umd.edu/mar/)

NGOs (e.g., Red Cross): Characteristics such as size, locations, resources, foreign links, political involvement and leanings

- Yearly data of UN houses from 1996 to 2005: United Nations Development Group: http://www.undg.org/index.cfm?P=214

- Yearly data of UN country development teams from 2003 to 2005: United Nations Development Group: http://www.undg.org/unct.cfm?module=CountryTeams\&page=Region\&R egionID=RAS

- USAID’s NGO Sustainability Index for Central and Eastern Europe and Eurasia (http://www.usaid.gov/locations/europe_eurasia/dem_gov/ngoindex/) 
- Duke University's resources Web page regarding NGOs (http://library.duke.edu/research/subject/guides/ngo_guide/index.html)

Religious organizations (e.g, churches, cults): Characteristics such as size, locations, resources, foreign links, political involvement and leanings, influence over population

- Adherency.com's religious statistics (http://www.adherents.com)

- Wikipedia Web page on religions by country (http://en.wikipedia.org/wiki/Religions_by_country)

Degree of religious freedom

- Hudson Institute's Religious Freedom Index (book publication imminent)

- U.S. Dept. of State Annual Report on International Religious Freedom (http://www.state.gov/g/drl/rls/irf/)

Other social movements (e.g., land reform, temperance): characteristics such as size, locations, resources, foreign links, political involvement and leanings

- Data sources as below

Social Safety Net: presence or absence, who is covered, magnitude of support

- U.S. Social Security Administration's Social Security Programs Throughout the World database (http://www.ssa.gov/policy/docs/progdesc/ssptw/)

Mechanisms for empowerment and creating opportunities

- World Economic Forum's Global Gender Gap Index (http://www.weforum.org/en/initiatives/gcp/Gender\%20Gap/index.htm)

- Avon Products, Inc.'s Worldwide Women's Poll and Empowerment Index (http://shop.avon.com/shop/landing/global_summit/summit_press4.html)

- United Nation's Human Development Report Gender Related Development Index and Gender Empowerment Index (http://hdr.undp.org/en/statistics/indices/)

Crime: type; distribution by region, social category, etc.; probability of personally experiencing a violent crime

- Yearly data of human development indices, including income, inequality, poverty, education, gender, family, demography, health, water, sanitation, nutrition, survival, political participation, technology, economic performance, trade, international aid, public spending, energy, pollution, environment, refugee, crime, gender empowerment, human rights from 1975-2008: United Nations Human Development Program: http://hdrstats.undp.org/countries/

- Data UNC JIN statistics and research sources: http://www.uncjin.org/Statistics/statistics.html

\section{Infrastructure}

Transportation:

Roads: network (location), capacity, condition, suitability for different types of transport (foot, animal, car, truck, military)

Vehicle ownership: type, concentration in population by location, class, etc. 
Other transportation: type (animal, foot); patterns in population by location, income, etc.

Water transport: network (location), capacity, condition, suitability for different types of transport

Rail transport: network (location), capacity, condition

Ports: locations, capacity, seasonal limitations

Air: number, location, and condition of airports; number and type of airplanes, control over airports and airplanes

- Same data sources as below

Communication:

Telephone networks: distribution of network, distribution of telephones

Television: channels/networks, access to channels, distribution of TVs, control over content

Internet: distribution of access, government (or other) control over content Print (newspapers, pamphlets): distribution, control over content

- Same data sources as below

- Internet, telecommunications, telephone, digital access index, main telephone lines:

http://earthtrends.wri.org/searchable_db/index.php?theme $=10$

Utilities:

Electrical, water: sources, distribution, capacity, reliability and vulnerability, access of population

- Water resources and freshwater ecosystems data from WRI: http://earthtrends.wri.org/searchable_db/index.php?theme $=2$

- Electricity data from WRI: same as above Fuel: type (gasoline, coal, etc.), sources, distribution, capacity, reliability and vulnerability, access of population

- Afghanistan data from World Bank Group from 1990 to 2006: http://ppi.worldbank.org/explore/ppi_exploreCountry.aspx?countryID=14 $\underline{9}$

- UN Energy statistics from UN Environment and Energy statistics: http://unstats.un.org/unsd/environment_main.htm

- Fuel data from WRI: http://earthtrends.wri.org/searchable_db/index.php?theme $=6$

- Mineral Resources of Afghanistan from UN Economics and Social Commission for Asia and the Pacific: http://unescap.org/publications/detail.asp?id=62

Note: Asia Society provides complete data for Afghanistan: http://www.asiasource.org/profiles/ap_mp_04.cfm 


\title{
Appendix 3 - LandScan Methodology
}

\author{
Methodology for Dasymetric Modeling for Building Synthetic Populations
}

\section{Summary}

The LandScan ${ }^{\mathrm{TM}}$ Global Population Database describes the 24-hour average (i.e., ambient) population distribution for every 30 arc-second (approximately $1 \mathrm{~km} \times 1 \mathrm{~km}$ ) grid cell covering the world. This database is produced through an innovative, flexible, and dynamically adaptable spatial model that refines census data utilizing remotesensing-derived earth observation data and geographic information systems (GIS) technology. The LandScan algorithm incorporates spatial data and imagery analysis technologies and a multivariable dasymetric modeling approach to disaggregate census counts within an administrative boundary. Since no single population-distribution model can account for the differences in spatial data availability, quality, scale, and accuracy as well as the differences in cultural settlement practices, LandScan population distribution models are tailored to match the data conditions and geographical nature of each individual country and region. Improvements to the population distribution data using new spatial data, imagery, census information, and algorithm enhancements are made annually. High-resolution imagery is used to refine population distributions and correct spatial data errors.

\section{Introduction}

The LandScan global population distribution model is a multilayered, dasymetric, spatial modeling approach that is also referred to as a "smart interpolation" technique.

Dasymetric modeling is analogous to areal interpolation [a technique by which data from one set of polygons are redistricted onto a set of overlapping, but nonhierarchical target polygons (Gregory 2002)] except that ancillary spatial data aids interpolation. Typically, in dasymetric mapping, source layers in the region are first transformed into a surface lattice. Rather than using purely mathematical algorithms to interpolate lattice cell values from known data associated with the original source zones, an ancillary data layer is added to the lattice and a weighting scheme is applied to cell counts according to known or derived density levels associated with values in the ancillary data.

In LandScan, the typical dasymetric modeling is improved by integrating and employing multiple ancillary or indicator data layers. The LandScan modeling process involves the collection of the sub-national level census counts for each country and primary geospatial input or ancillary datasets, namely land cover, roads, slope, and urban areas and village locations and high resolution imagery analysis; all of which are key indicators of population distribution. Relationships between any of these datasets and population distribution are not globally uniform. For example, roads play a critical role in human settlements, independent of other forms of transport. However, residential population density in proximity to major roads varies significantly across the world. Such as, residential population is much higher near primary roads in south Asia countries, where 
the opposite is true in the United States. Similarly, the tendency to reside on steeper slopes rather than on gentler (or flat) terrain is generally a function of the abundance of available areas with gentler slope as well as local climatic regimes. Based on this variability in cultural and settlement geography, each country is considered to have a unique set of settlement characteristics.

Within each country, the population distribution model calculates a "likelihood" coefficient for each LandScan cell and applies the coefficients to the census counts, which are employed as control totals for appropriate areas. For example, in the United States, census blocks serve as the polygonal unit or control population but generally census data are aggregated to and are associated with an administrative boundary. These administrative boundaries are divided into finer grid cells $(1 \mathrm{~km})$, and each cell is evaluated for the likelihood of being populated based on its geospatial characteristics. Based upon the land cover category (such as agricultural or commercial) and upon the socioeconomic and cultural understanding of an area, cells are preferentially weighted to accommodate the likelihood of the presence of workers. This represents a 24-hour average population rather than just a residential population. The total population for that area is then allocated to each cell proportionally to the calculated population coefficient, which represents the likelihood of any cell being populated. The resultant population count is an ambient or average day/night, population count.

\section{Ambient Versus Residential Population}

Commonly available census population data are severely constrained both in space and time and do not capture the population dynamics as functions of space and time. Indeed, from a spatial perspective, census data are limited by census accounting units, such as blocks, where often there is great uncertainty about the spatial distribution of residents within those accounting units. This is particularly true in suburban and rural areas, where the population is dispersed to a greater degree than in urban areas. From a temporal perspective, census counts represent "residential" or "nighttime" population, and their use in a daytime event is highly inaccurate. Because of this uncertainty, there is significant potential to misclassify people with respect to their location in relation to hazards, for example, pollution sources. Consequently, it becomes extremely challenging to determine, with the required accuracy, whether certain subpopulations are more likely than others to get differential environmental exposure. To a large degree, these limitations can be overcome by developing population data with a finer resolution in both space and time at sub-census levels. ORNL has developed a very high resolution (90 meter) diurnal (nighttime residential and daytime) population distribution database for the U.S. (LandScan USA). However the goal of LandScan is to produce a distribution of an "unwarned” or 24-hour average population.

The LandScan data represents an average or ambient population that integrates diurnal movements and collective travel habits into a single measure. This is desirable for purposes of emergency response. Consider, for example, the hypothetical case of a cell with a major, multilane highway passing through a desert. If a nuclear, biological, or chemical (NBC) release contaminates the cell, the potential exists for many lives to be at 
risk even though no one lives there. Most official census counts, if available at such fine resolution, would show zero population because most national censuses are concerned with residential population based primarily on where people sleep rather than where they work or travel. In the LandScan procedure, population will be apportioned to the cell based on the presence of ambient highway traffic. Consider another cell containing a large agricultural field and no houses. Most censuses would place farm workers in their village residences and record zero populations for their fields. Yet, a few lives are at risk in the fields, depending on when the NBC release occurs. Hence, our procedure will show population in the agricultural cell (based on the region's agricultural practices) and a slightly reduced population in the village to suggest, albeit imprecisely, the collective time that some villagers are in their fields rather than their homes. For certain nations, even arid grassland cells will have sparse populations assigned to simulate the movements of nomads and other herders. The same can be said of factories, airports, and other places of work and travel.

\section{Census Counts}

All population counts, even the most sophisticated high-resolution official censuses of advanced nations like the United States, are stochastic estimates. Accuracy and precision are limited by the census takers' access to homes and even to whole neighborhoods; by the census takers' understandings of personal work and travel habits; and by the frequency with which censuses can be undertaken. These limits are exacerbated in many nations by lack of resources and, all too often, outright manipulation of census figures to meet political objectives. In addition, many nations are reluctant to release detailed census counts. The variable quality of census figures from country to country presents a major challenge to global population distribution efforts such as LandScan. The International Program Center (IPC) at the U.S. Bureau of Census provides ORNL updated census data at the sub-national administrative level. Individual nations conduct their census at different intervals, thus "new" census information is acquired by the IPC each year. For inter-census years, the IPC is tasked for producing the midyear population projection for each subnational administrative area. These projections become the “official” U.S. estimate of international populations and are supplied to ORNL as a spreadsheet. Certain national census estimates provided by other organizations (e.g., United Nations) may differ from those produced by IPC. However, all census counts incorporated into the models used by LandScan are produced by the IPC.

\section{Input Data and Data Management}

In addition to census data, large volumes of high-resolution satellite-derived spatial data as well as traditional mapping data are used during the LandScan modeling process and during the verification and validation $(\mathrm{V} \& \mathrm{~V})$ of the model results. Datasets include

- $1 \mathrm{~m}$ and 5 m satellite imagery (CIB01 and CIB05),

- High Resolution Commercial Satellite Imagery (Ikonos and Quickbird),

- $\quad$ Derived land cover [MODIS]

- $\quad$ Shuttle Radar Topography Mission [SRTM] formats 1 and 3,

- $\quad$ Digital Terrain Elevation Data [DTED] levels 1 and 2, 
- Vector Smart Map [VMAP] levels 1 and 2,

- Urban Vector Smart Map [UVMAP] data,

- GeoCover Land Cover data,

- Coastal Change Analysis Program [CCAP] Land Cover Data,

- $\quad$ National Land Cover Database [NLCD] data, and

- $\quad$ Compressed ARC Digitized Raster Graphics [CARDG] data.

\section{Spatial Data Input Variables}

\section{Vector Data}

- Transportation networks (i.e., roads, railroads, airports, and navigable waterways) are primary indicators of population. As a single indicator, roads are preferred because of their vital role in human settlements with or without other forms of transport.

- Populated places include polygonal outlines of urban areas as well as point locations of smaller towns and villages. Attributes for populated places data may provide the name but not the population associated with each location.

\section{Raster Data}

- Elevation and slope are important variables in calculating the LandScan population probability coefficient because most human settlements occur on flat to gently sloping terrain. Even in regions noted for hillside settlement, relative measures of slope may correspond (inversely) with population density. Rather than calculating an average slope value for each 30 second cell which may mask many small relatively flat areas, a slope "index" is produced by processing higher resolution elevation data into discrete slope categories. This methodology captures the percentage of land preferable for habitation within each 30 arcsecond cell.

- Land cover is perhaps the best single indicator of population density. In most regions population would range from extremely low densities in desert, wetlands, ice, or tundra land cover to high densities in developed land cover associated with urban land use. Arid grasslands, forests, and cultivated lands would range in between. LandScan analysts assign relative weights to each land cover type based upon the region's cultural settlement patterns and agricultural intensity practices, and employ these weights in calculating the probability coefficient for each cell. Most land cover data used in LandScan was processed using Landsat Thematic Mapper (TM) at a resolution of $30 \mathrm{~m}^{2}$. For certain areas where no TM-derived data is available, land cover information is derived from the Moderate Resolution Imaging Spectroradiometer (MODIS) at a nominal $1 \mathrm{~km}^{2}$ resolution. Even at 1 $\mathrm{km}^{2}$ resolution, land cover can be a good indicator of relative population density, and its efficacy improves as resolution approaches the typical size of individual home sites. 


\section{High-Resolution Imagery}

- High-resolution imagery is incorporated throughout the LandScan population distribution modeling process and facilitates the assignment of relative weights to land-cover and slope categories for each particular region. Imagery is used to update or correct existing land-cover information and includes capturing the spatial extents of growing urban areas as well as correcting spatial data anomalies such as town and village precision errors or omissions.

\section{Exclusion Areas}

- $\quad$ Some areas, such as cells whose land cover are composed of $100 \%$ water as well as islands with no permanent inhabitants, are excluded from the ambient population distribution although there may be people in these locations for a short period of time.

\section{Administrative Boundaries and Coastlines}

- Considerable effort is required to reconcile the positional accuracy of diverse global databases, and mismatches among databases are most conspicuous on administrative boundaries and coastlines. Accurate administrative boundaries (especially at the $2^{\text {nd }}$ order-e.g., subprovince) that are associated with the census data as control totals remain problematic for much of the world. Many digital versions of sub-national boundary information exist, but none are authoritative. In fact, when many of these boundaries are overlain on geo-rectified imagery it can be seen that spatial precision and/or attribute correctness is lacking. ORNL continually searches for, and in some cases creates, more precise $2^{\text {nd }}$ order or higher administrative boundaries. The same precept hold true for coastlines. Typically, a coastline differs somewhat from the related line representing the seaward boundary of administrative units. For this reason, all coastal administrative boundaries used in LandScan are extended seaward to avoid omitting any coastal land.

\section{Verification and Validation (V\&V)}

Verification of spatially explicit global population databases is inherently limited by the difficulty of establishing a suitable reference database for purposes of comparison. The ideal reference database would be actual census counts for sample areas at the same resolution or finer resolution than the database being evaluated, in this case $30 " \times 30 "$ cells. A traditional validation or ground-truthing of the LandScan data is implausible due to the tremendous resources necessary for such a process as well as the logistical issues of replicating a "census" for a foreign state. Such a comparison may be feasible for certain countries that collect and disseminate high resolution (e.g., block or tract level) census data, but this applies only to urbanized areas of the United States, certain other advanced countries, and a few less developed countries. Unfortunately, suitable reference data are least likely to be available for unstable hostile regions where improved 
population distributions are most needed for government planning, i.e., to assess risks to military and diplomatic personnel and civilian populations or to provide humanitarian relief.

However, there are some measures by which qualitative assessments of the LandScan data may be made. By examining the characteristics (scale/resolution, date, etc.) of the various LandScan model input data layers, relative comparisons are possible that enable a general “confidence level” to be considered for assorted processing tasks. V\&V activities include tests of (a) consistency with ancillary data and/or (b) surrogate analysis of similar areas. The accuracy of spatial models such as LandScan is tied to the resolution and accuracy of the data layers used in the model. New digital spatial data and imagery are created on a daily basis and the LandScan model attempts to incorporate as much new spatial data as time and budget constraints allow. However, these data are often not consistent in scale, resolution, spatial precision, or attribute accuracy on a global basis, and in fact, may vary considerably within a country. For this reason, the "confidence level” of the LandScan population distribution varies from country to country.

LandScan receives all population and demographic information from the International Program Center, Bureau of the Census (IPC). The IPC acquires foreign census information and further validates and extrapolates the population information to produce a midyear estimate at the province or sub-province level. LandScan uses a normalization process that guarantees that the population counts distributed throughout an administrative boundary will reflect exactly the number reported by IPC for that coinciding area. However, individual countries conduct their census at diverse intervals and to various level of detail. The mid-year estimates produced by IPC may not reflect certain population movements [e.g., migrations, internally displaced persons (IDPs), refugees, etc.] since the last official census conducted by a country. In addition, the administrative boundary level by which the census data is distributed varies considerably in size and spatial precision from country to country. Generally, the LandScan model will reflect population distributions more accurately for those areas with smaller and spatially precise administrative boundaries.

Most digital spatial data and imagery used in the LandScan model are produced by or for the NGA and supplied as input to the LandScan model. These data (e.g., land cover, elevation/slope, roads, urban areas, village locations, etc.) vary considerably in resolution and accuracy throughout the world. For example, derived land cover may vary in type and scale from the MODIS at a nominal $1 \mathrm{~km}^{2}$ resolution to that derived from the Landsat Thematic Mapper (TM) at a resolution of $30 \mathrm{~m}^{2}$ to that derived from submeter resolution imagery analysis. However, producing accurate land-cover data at a high resolution is a difficult and time-consuming process, and for many areas of the world the land-cover data reflect imagery that is now many years old. Thus many rapidly growing urban areas are not adequately captured in the land-cover data and must be interpreted manually using high-resolution imagery. Vector information included in datasets such as the VMAP1 series include data such as roads, urban boundaries, and village locations and are digital representations of a paper map series (i.e., 1:250,000 scale). Thus the accuracy and currency of the VMAP data not only depends upon the raster and vector processes used 
to produce the VMAP data, but also upon the accuracy of the original compilation of the paper map. Therefore, data precision and completeness varies by the individual maps and can be seen both between and within various VMAP data tiles. Generally, the LandScan model will reflect population distributions more accurately for those areas with recent and higher spatial resolution.

\section{Results}

As access to increasingly better input data from the NGA (and its predecessors) has improved, so has the modeling and model results validation. Indeed, the use of quality input datasets results in a superior population distribution. ORNL has included the use of very high-resolution imagery during the model results validation process, and each year, several entire countries are validated by using $1 \mathrm{~m}$ and $5 \mathrm{~m}$ imagery products and by conducting photographic interpretation of the countries. As part of the validation effort, all countries are provided with photographic interpretation of the data. This effort ensures that the model has appropriately assigned population to cells. It also locates previously undocumented village locations across entire countries and helps to locate the true urban zones. Other validation datasets are used in a similar fashion. ORNL uses local landcover datasets developed by organizations in-country to aid in the validation of the LandScan modeling results. 


\section{Appendix 4 - Narrative Description of Key Data Sources}

\section{Granular Placement of Social Atoms-The National Geospatial-Intelligence Agency}

The National Geospatial-Intelligence Agency (NGA) is responsible for the USG programs in GEOINT ( GEOspatial INTelligence). GEOINT is an intelligence discipline comprising the exploitation and analysis of satellite information to describe, assess, and visually depict physical features and geographically referenced activities on the Earth. As a beginning, we will describe the ability to place social atoms geospatially within OCONUS regions of U.S. interest.

The LandScan ${ }^{\mathrm{TM}}$ dataset comprises a worldwide population database compiled on a $30 " \times 30 "$ latitude/longitude grid. Outside the United States, the census region corresponds to grids about one kilometer on a side. Census counts (at subnational level) were apportioned to each grid cell based on likelihood coefficients, which are based on proximity to roads, slope, land cover, nighttime lights, and other all-source information. LandScan has been developed as part of the Oak Ridge National Laboratory (ORNL) Global Population Project for estimating ambient populations at risk.

The LandScan dataset files are available via the internet in ESRI grid format by continent and for the world, and in ESRI raster binary format for the world. Figure A4-1 provides a country-by-country assessment of the country coverage.

Figure A4-1. The LandScan quality map represents data quality on a nation by nation basis.

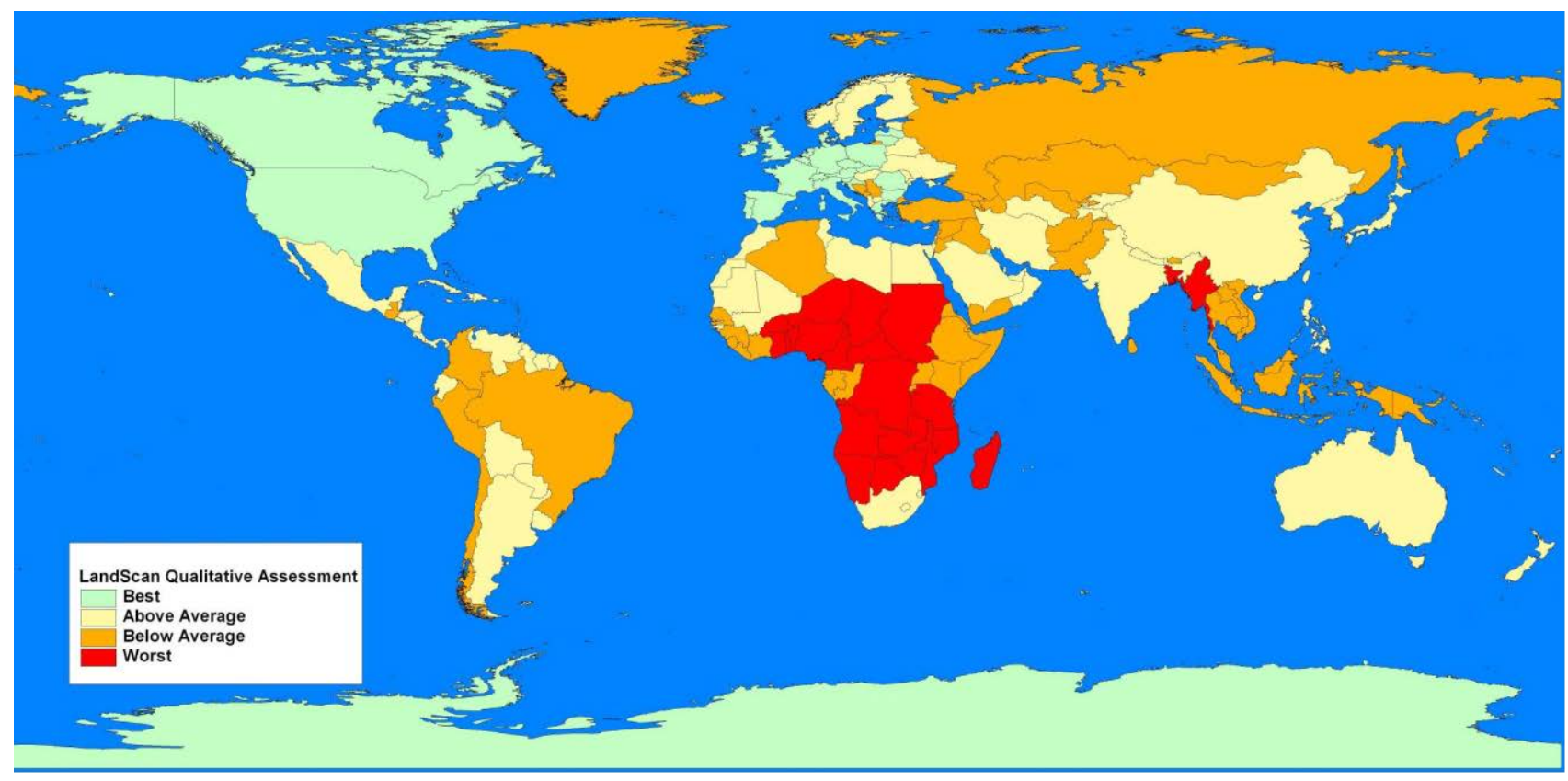


LandScan datasets are released annually. In the most recent (fourth) version, NASA's earth observing system (EOS) moderate resolution imaging spectroradiometer (MODIS) land-cover database was used for input data. The MODIS land-cover data replaced an earlier AVHRR-derived global land-cover database. The land-cover data was refined substantially using National Imagery and Mapping Agency's (NIMA) VMAP-1, CIB, and/or scanned maps, CADRG/ADRG. Accurate administrative boundaries are an integral part of the LandScan population distribution modeling process. ORNL received or developed an additional 700 international second-order administrative boundaries that were used for population distribution. For certain countries, the precision of the administrative boundaries were improved. These boundaries coincide with new census counts provided by the International Programs Center (IPC) of the United States Bureau of the Census and represent the most recent census for all countries. New and revised National Imagery and Mapping Agency's (NIMA) VMAP-1 tiles were incorporated, continuing the improvement of road networks, populated places, and waterbodies. Also, new and revised NIMA's DTED-1 tiles were added, thus enabling us to calculate how much land within each cell is suitable for settlement. ORNL made extensive use of NIMA's Controlled Image Base (CIB) imagery for verification and validation and for refining urban built-up areas. Additional algorithm refinements were developed and implemented for the population models.

\section{The Afghan Example}

An example of the LandScan results for our Afghan case study is shown in Figure A4-2. Even though the data quality for Afghanistan is described as below average, census data is currently being collected in Afghanistan, illustrating that placement of social atoms within one kilometer grids is feasible even in areas that are data sparse. 
Figure A4-2. 2002 Afghan Population Density

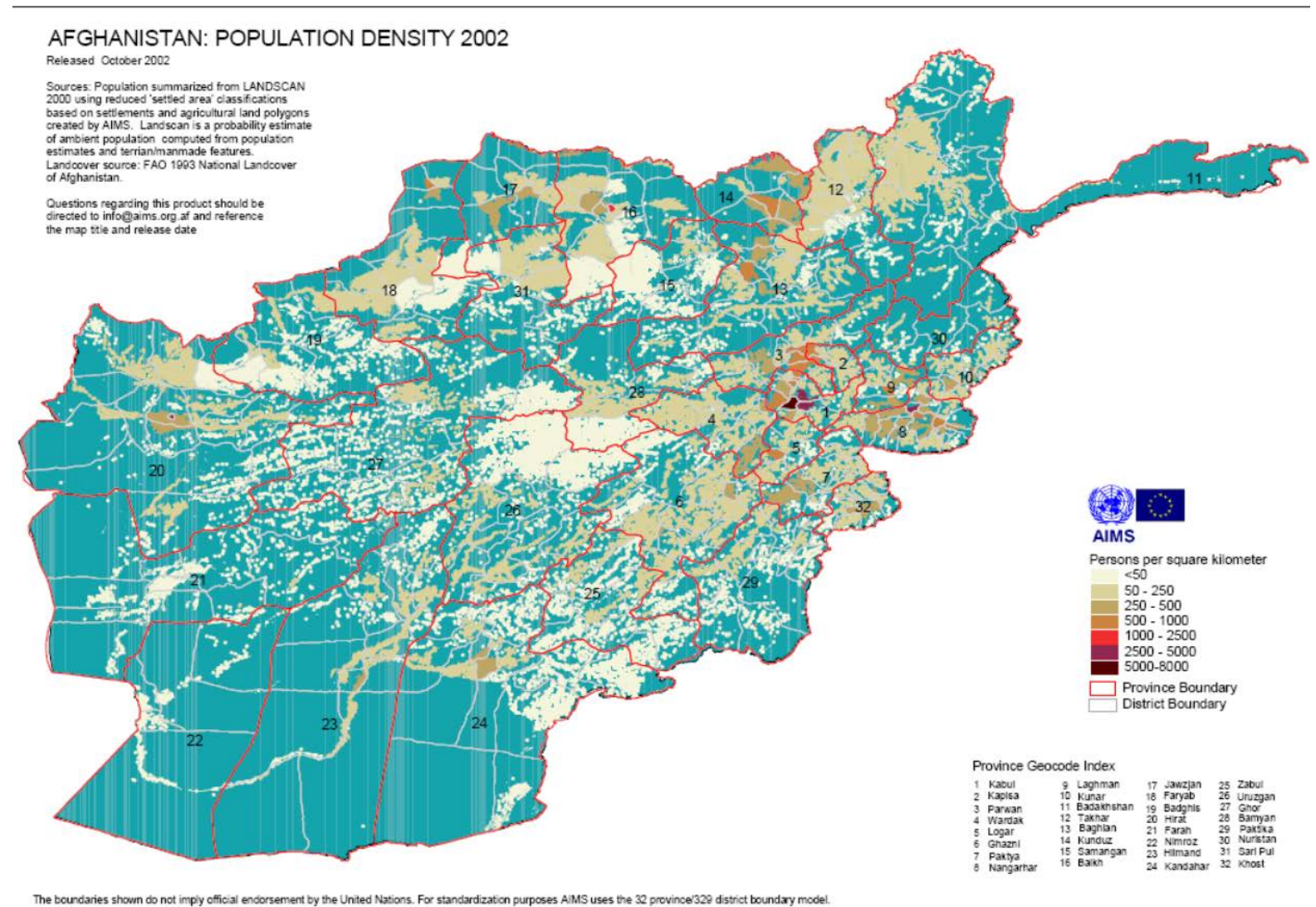

\section{Data Description of Nongovernmental Agencies}

To address the location, efforts, and nature of nongovernmental agencies (NGOs), informal groups, and indicators of actions and intentions, we will describe long-term data collection programs that show the feasibility of obtaining the required data and then show selected examples for the Afghanistan case study.

\section{Database of Archives of Non-Governmental Organizations (DANGO)}

The database of archives of NGOs since 1945, DANGO is a UK on-line, free resource, enabling researchers to identify NGOs and then access both existing and new information about the content, location, and accessibility of archival holdings relating to those bodies. DANGO is an Arts and Humanities Research Council (AHRC)-funded project that will be maintained until at least 2012. Information can be accessed through the DANGO Web site where the prototype database is updated on a daily basis.

Database users can search for NGOs in various ways, such as by name and areas of interest. Links to on-line catalogues are provided, as well as summaries of information held elsewhere, like the NRA's catalogue collection, with the aim of bringing together 
everything that is currently in the public domain. All the essential information for researchers such as location, extent, and access conditions is also provided.

\section{Office of the United Nations High Commissioner for Refugees}

Because regional stability issues often revolve around human migration (Darfur, Iraq, Afghanistan, Bosnia, Kosovo, Israel-Palestine, Lebanon), NGO activities in these areas are important additions to direct humanitarian activities. The following provides an overview of the activities of Global Migration Group (GMG) members in the fields of migration- and development-related policy research, data-collection and publications.

The GMG is an inter-agency group bringing together heads of agencies that seeks to promote the wider application of all relevant international and regional instruments and norms relating to migration, and to encourage the adoption of more coherent, comprehensive, and better coordinated approaches to the issue of international migration. The GMG consists of 10 organizations that are actively involved in international migration and related issues. The following four organizations are the most important for the purposes of the ASCES and are described in the sections below:

International Labor Organization (ILO)

United Nations Department of Economic and Social Affairs (UNDESA)

United Nations Population Fund (UNFPA)

World Bank

\section{International Labor Organization (ILO)}

Collection of data and statistics on the international migration of workers forms part of a comprehensive body of labor and employment information compiled by the office. This makes it possible to relate migration information to broader employment and labor market issues facing countries. ILO's work is divided into several areas:

- Contribution to methodology and guidelines for developing migration statistics: The ILO has developed comprehensive manuals and guidelines for collecting migration statistics. The ILO serves on interagency expert committees on improving migration and related statistics. It has developed a migration module to be attached to existing household surveys on the labor force, and is testing it in certain countries. Particular attention is paid to the measurement of migrant remittances. The goal is to create questions that effectively measure various dimensions of labor migration in both source countries and destination countries and can be periodically added to household surveys. The module is currently being tested in both Armenia and Thailand.

- Global estimates on labor migration and trafficking: ILO made an estimate of the number of migrant workers globally and regionally based on UN estimates of migrant stocks for the year 2000. These estimates are 
currently being updated. The ILO (Program for Promotion of the Declaration) has made a minimum estimate of the incidence of forced labor at the global and regional levels, including those trafficked.

- Databases on labor migration information and statistics: The online International Labor Migration Database, which has been operational since 1999, consists of statistics on labor migration for 86 member states, compiled in cooperation with EUROSTAT for Europe and through a questionnaire sent to other countries.

The information collected and research outputs are disseminated through special publications and reports, working papers (mainly the series on International Migration Papers and Perspectives in Labor Migration), presentations at various conferences/workshops, newsletters, and the ILO Web. Most studies and publications can be accessed and freely downloaded from the MIGRANT Web site.

\section{United Nations Department of Economic and Social Affairs (UNDESA)} The Population Division of UNDESA monitors global international migration trends and policies. It carries out research and publishes reports on international migration and development and maintains various databases. In the context of preparing the estimates and projections of the world population, it produces estimates of net migration flows. The following UNDESA compilations are currently available:

- International migrant stock: This database contains estimates of the number of international migrants by sex in each country or area of the world as of 1 July 1960, 1965, 1970, 1975, 1980, 1985, 1990, 1995, 2000, and 2005. The estimates are derived mostly from data on the number of foreign-born persons enumerated by censuses. Estimates of the number of female migrants and refugees are also included.

- Net migration estimates: Estimates and projections of net international migration for each country of the world and for each five-year period from 1950 to 2050 are available as part of the set of population estimates and projections released under biennial revisions of World Population Prospects. The most recent is the 2004 Revision.

The Population Division publishes the International Migration Report on a periodic basis. The report presents relevant data on international migration trends and policies for each country of the world. It also issues a biennial report on World Population Policies providing an overview of governments' views and policies on population, including those relative to international migration. The 2005 edition is the most recent, covering the period 1976 to 2005. 
The Population Division carries out research on the various aspects of international migration, including its demographic implications. The report entitled Replacement Migration, for instance, explores the potential impact of international migration in countering population ageing.

The UNDESA Statistics Division collects and disseminates statistics on stocks and flows of international migrants. It uses the Population Census Questionnaire to compile census data on the number and characteristics of foreign-born and foreign persons enumerated by censuses. The data collected are disseminated via the Demographic Yearbook. As of early October 2006, 71 countries had reported data on the native and foreign-born population by age, sex and urban/rural residence for censuses taking place from 1985 to 1994, and 62 countries had done so for censuses carried out from 1995 to 2004. The population by country of citizenship and sex had been reported by 71 countries for censuses occurring from 1985 to 1994 and by 53 countries for censuses with dates ranging from 1995 to 2004. The foreign-born population by country of birth, age, and sex had been reported by 61 countries with censuses taking place between 1985 and 1994 and by 47 countries with censuses occurring between 1995 and 2004. The foreignborn population enumerated in a country who had lived abroad five years before the census, classified by country of birth, age, and sex, was reported by 17 countries each for the periods 1985-1994 and 1995-2004. The economically active foreign-born population by occupation, age, sex, and urban/rural residence was reported by 29 countries with censuses dating from 1985 to 1994 and by 24 censuses dating from 1995 to 2004. 


\section{United Nations Population Fund (UNFPA)}

UNFPA supports collection, analysis, and dissemination of good-quality and timely age- and sex-disaggregated migration data for evidence-based decisionmaking and policy formulation. UNFPA supports operational research that fosters increased understanding of the migration process and addresses the needs of migrants, especially female migrants.

\section{World Bank}

The World Bank is developing its research and statistical activities with a focus on enhancing the quality of the policy debate on migration and development. These efforts include activities on data collection, processing and reporting on migration and remittances. The analytical work is exploring in detail issues related to gender, subregional migration, poverty, social security, and trade, among other topics.

As part of its research program, the Bank is piloting different instruments for collection of information on migrants and their behavior. These include

- separate migration modules in household surveys, such as those already implemented in Morocco, Guatemala, and Ghana; and

- alternative sampling designs for the migrants' survey to better capture the migrant population.

The database was recently expanded to include information on the age of migrants and the location of their education. A specific focus on the health sector includes data on the bilateral migration of medical personnel between developing countries and OECD countries.

\section{International Programs Center Demographic and Socioeconomic Research Services}

The International Programs Center (IPC) of the U.S. Bureau of the Census conducts studies covering a wide range of demographic, socioeconomic, and health issues. These include

- population projections and trends for all countries of the world and selected subnational areas,

- tracking and projecting HIV/AIDS prevalence and assessing the demographic consequences,

- economic and social status of populations in transition to market economies, and

- the role and status of women. 


\section{Afghanistan Case Study}

The development of the data for the Afghanistan case study draws on the above generally available global databases. However, the United Nations Office for Coordination of Humanitarian Affairs (UNOCHA) has undertaken a program to synthesize available data for Afghanistan, and we will draw from their synthesis here.

The UNOCHA effort had its origin in the Project Management Information System (ProMIS) that was started in Islamabad, Pakistan under direct supervision of the U.N. Food and Agriculture Organization (FAO) in 1997. The purpose was to enhance the capacity of UN Agencies, NGOs, and the donor community to undertake analysis and planning for operational activities as well as common programming for Afghan rehabilitation and development activities. The ProMIS data center in Islamabad produced, maintained, and distributed data and information related to Afghanistan.

After 11 September 2001, UNOCHA proposed to utilize the information developed by ProMIS in conjunction with a new Humanitarian Information Center (HIC). An agreement between the U.N. Development Program (UNDP), FAO, and UNOCHA allowed the three agencies to combine resources for delivering information management services to the humanitarian community. This new project was named HICFA (Humanitarian Information Center for Afghanistan).

Subsequently the Afghanistan Information Management Service (AIMS) was established in late 2001 as a joint venture between UNDP and UNOCHA through the merging of two projects - the Humanitarian Information Center for Afghanistan (HICFA) and ProMIS. At the same time AIMS established regional offices in collaboration with the U.N. Assistance Mission in Afghanistan (UNAMA) in five Afghan cities.

Throughout 2002 and into 2003, AIMS continued to provide a variety of GIS, database, and information management services to the government and humanitarian community. In 2003 AIMS became a Directly Executed (DEX) project of UNDP, administered and managed by UNDP and reporting to the Resident Representative with a new mandate of building information management capacity in the government of Afghanistan.

\section{Example Data}

The AIMS data profiled each of the 388 Afghan districts, creating a series of district profiles. Each profile lists the province and district; representative villages; ethnic composition; returnee population; leadership ethnicity and party; government departments; leadership; NGOs including UN, national, and international groupings; employment; housing stock; and access to basic social services. An abbreviated example of a district profile is reproduced below. 
Table A4-1. Example district profile

\begin{tabular}{|c|c|c|c|c|c|c|c|}
\hline \multicolumn{8}{|c|}{ DISTRICT PROFILE } \\
\hline Data Collection Period: & \multicolumn{7}{|c|}{9 October 2004 to 15 October 2004} \\
\hline \multicolumn{2}{|l|}{ PROVINCE } & \multicolumn{6}{|c|}{ Farah } \\
\hline \multicolumn{2}{|l|}{ DISTRICT } & \multicolumn{6}{|l|}{ Gulistan } \\
\hline \multicolumn{2}{|l|}{ Villages Visited } & \multicolumn{6}{|c|}{$\begin{array}{l}\text { Dehak Raswa, Midan Jabha, Siab, Gandachah, Kotalaka, Dajahangir, Tarkhi, Dehsabz, Takht } \\
\text { Safid, Ghozini, Qala Sorkhkar, Chinigay, Faiz Abad, Pasaw Helalzai, Baghroad, Saidal, } \\
\text { Tangitaw, Kosha, Gudai, Shirsorkh, Ahmad Abad, Dehak Bala, Qumbaran, Saikosha, Pasaw, } \\
\text { Kham Shawlat, Tangi Naw, Shatoot, Larti, Baghdasht, Nal, Ghorma, Guldare, Sang Sorakh, } \\
\text { Khangek, Kokcha, Qalai Kohna and Ghormash }\end{array}$} \\
\hline \multicolumn{2}{|l|}{ ESTIMATED POPULATION } & \multicolumn{6}{|c|}{ 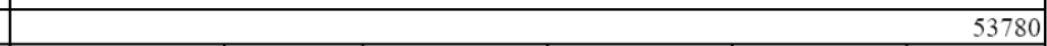 } \\
\hline \multicolumn{2}{|l|}{ ETHNIC COMPOSITION } & Pashtun (80\%) & & Tajik (20\%) & & & \\
\hline \multicolumn{3}{|c|}{ ESTIMATED RETURNEE POPULATION } & \multicolumn{5}{|r|}{704} \\
\hline \multicolumn{8}{|c|}{ AUTHORITY } \\
\hline District Governor & Name & M. Qasem Majbor & Ethnicity & Pashton & Party & Mahaz-e-Mili & \\
\hline D. District Governor & Name & Jan Mohamad & Ethnicity & Pashton & Party & Parcham & \\
\hline Functioning Departments & \multicolumn{3}{|c|}{ Director Name } & \multicolumn{2}{|c|}{ Nationality } & \multicolumn{2}{|c|}{ Party } \\
\hline Attorney & \multirow{2}{*}{\multicolumn{3}{|c|}{$\begin{array}{l}\text { Amir Mohd } \\
\text { M. Hashem }\end{array}$}} & \multicolumn{2}{|c|}{ Pashton } & \multicolumn{2}{|c|}{ Hizb-e-Khalq } \\
\hline Statistic & & & & \multicolumn{2}{|c|}{ Pashton } & \multicolumn{2}{|c|}{ Afghan Milat } \\
\hline Communication & & \multicolumn{2}{|c|}{ Tajik } & Hizb-e- & Chalq \\
\hline Finance & \multicolumn{3}{|c|}{ Sarajuddin } & Taj & & Hizb-e- & Shalq \\
\hline Rights & & hulam Mohaiuddin & & Pash & hton & Hizb-e- & Shalq \\
\hline Agriculture & & Sarajuddin & & Pash & hton & Hizb-e- & Chalq \\
\hline & & HUMANITAI & RIAN ACT & IVITIES & & & \\
\hline UN Organizations & UNICEF & Activities: Health a & and Educatio & & & & \\
\hline INGOs & DACAAR & Activities: Water S & supply & & & & \\
\hline INGOS & ALISIE & Activites: Clinic $\mathrm{Cc}$ & onstruction, & Wells, Road Grav & velling and Shel & & \\
\hline NeO & $\mathrm{CHA}$ & Activities: Health & & & & & \\
\hline NNGOS & $\mathrm{ADA}$ & Activities: Home-B & Based School & 1, Kariz Cleaning & and Agriculture & & \\
\hline National Programs & & & & & & & \\
\hline & & EMP & LOYMENT & & & & \\
\hline Type of Employment & Farmer & Husbandry & Daily Wage & $\begin{array}{c}\text { Seasonal within } \\
\text { Afghanistan }\end{array}$ & $\begin{array}{c}\text { Seasonal } \\
\text { outside } \\
\text { Afghanistan }\end{array}$ & Others & \\
\hline & Yes & No & No & Yes & Yes & & \\
\hline $\begin{array}{l}\text { Comments: People are in deb } \\
\text { of employment }\end{array}$ & due to the lack & of employment in th & e area. Some & e returnees sold th & heir belongings & $s$ a result of dro & ight and lack \\
\hline & & HOUSE AI & ND LAND I & ISSUE & & & \\
\hline House Recovered & \begin{tabular}{|l} 
House \\
Destroved
\end{tabular} & $\begin{array}{l}\text { House } \\
\text { Confiscated }\end{array}$ & Comments: & & & & \\
\hline Yes & Yes & & Destruction & n was seen by $50^{\circ}$ & & & \\
\hline & & ACCESS TO BAS & SIC SOCIAI & L SERVICES & & & \\
\hline A.Water & Acces & s to Source & Proble & m of Access & Comments: & & \\
\hline & Kariz an & d Shallow well & Low & water table & & & \\
\hline B. Education & $\begin{array}{l}\text { Facilities for } \\
\text { boys }\end{array}$ & Facilities for & $r$ girls & Problem & of access & Comments: & \\
\hline & Yes $(10 \%)$ & No & & Lack of School & Is and teachers & & \\
\hline C. Health & $\begin{array}{l}\text { Facilties } \\
\text { available }\end{array}$ & $\begin{array}{l}\text { Facilities for } \\
\text { women }\end{array}$ & Proble & of access & Comments: & & \\
\hline & No & No & Lack & of Clinics & $\begin{array}{l}\text { There is only o } \\
\text { equipped. }\end{array}$ & e clinic with, br & poorly \\
\hline
\end{tabular}


These district profiles can be coalesced into general maps of NGO identification, location, and activities necessary for the ACSES modeling effort. A 2003 example from the Afghan Information Management Services, (http://www.aims.org.af/) ,is reproduced in Figure A4-3.

Figure A4-3. Local NGO sectoral activities from http://www.aims.org.af/

\section{Local NGOs Sectoral Activities in Afghanistan - February 2003}

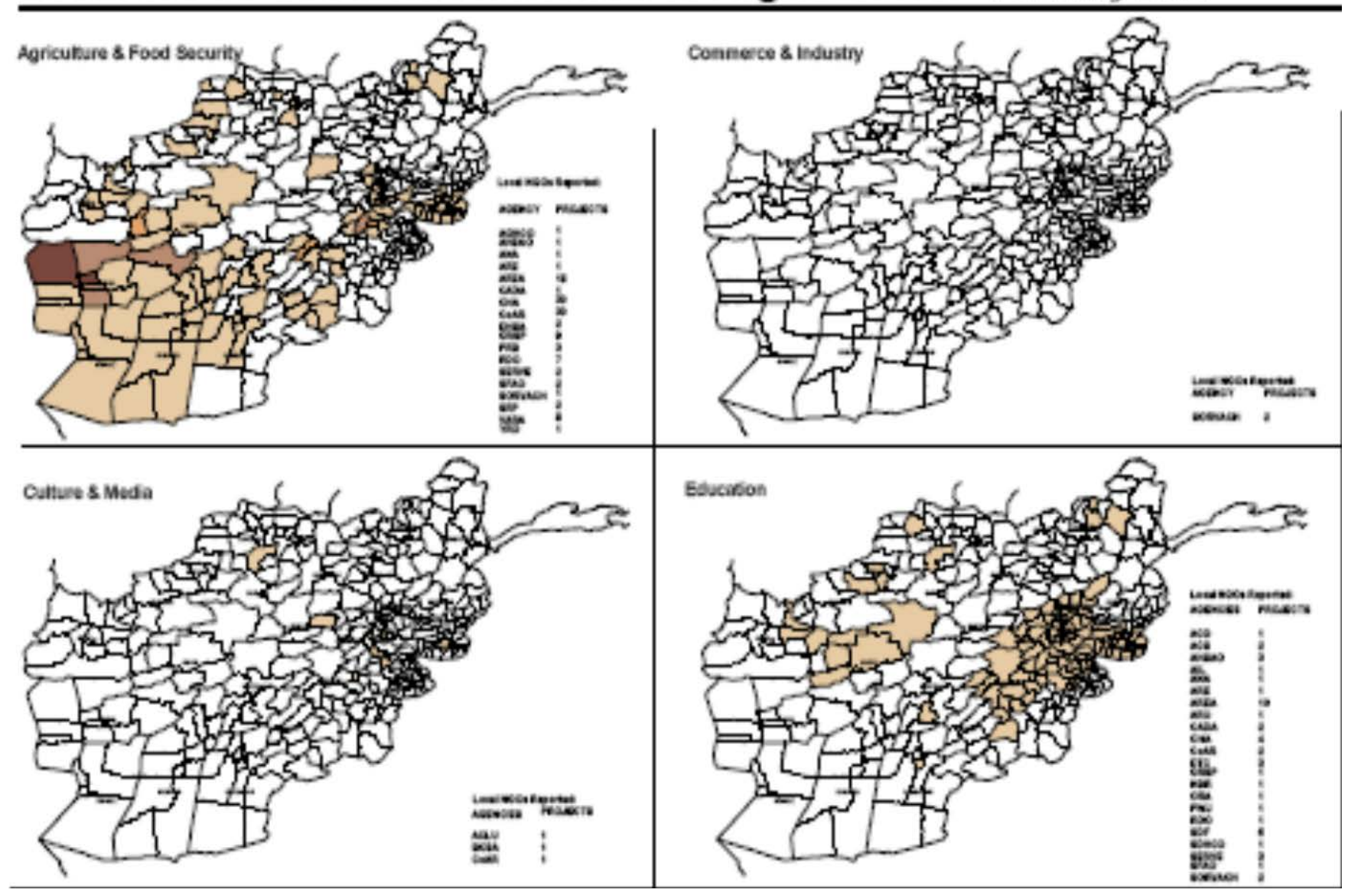

Considering the data programs gathering NGO and leadership data on a global scale, it appears feasible to identify, enumerate, and distribute people, organizations, and governmental institutions through the simulation system according to the communities and mixing groups necessary to develop causal factors and actionable future courses of action. However, once the agents are distributed among the communities and mixing groups, we must rely on global data-collection efforts to populate the agents with beliefs. 


\section{Worldwide Surveys of Community and Mixing-Group Beliefs}

The programs available to develop and maintain databases of beliefs, attitudes, and intentions for the assumed communities and mixing groups must be

- worldwide in scope,

- collected with common and consistent methodology, and

- maintained with known currency.

To illustrate what is available, we will examine an example of a worldwide sampling program.

\section{Gallup World Poll}

The Gallup World Poll maintains the capability to survey attitudes and beliefs in the countries listed below. The Gallup Organization is entirely responsible for the management, design, control, and funding of the poll. Any individual, institution, or governmental agency may access the database, regardless of nationality.

Table A4-2. Gallup World Poll coverage from www.Gallup.com

\begin{tabular}{|c|c|c|c|c|}
\hline Afghanistan & Colombia & Ireland & Netherlands & Spain \\
\hline Albania & Costa Rica & Israel & New Zealand & Sri Lanka \\
\hline Algeria & Croatia & Italy & Nicaragua & Sudan \\
\hline Angola & Cuba & Jamaica & Niger & Sweden \\
\hline Argentina & Cyprus & Japan & Nigeria & Switzerland \\
\hline Armenia & Czech Republic & Jordan & Norway & Taiwan, Province of \\
\hline Australia & Denmark & Kazakhstan & Pakistan & China \\
\hline Austria & Dominican Republic & Kenya & Palestinian Territory & Tajikistan \\
\hline Azerbaijan & Ecuador & Kosovo & Panama & Tanzania \\
\hline Bangladesh & Egypt & Kuwait & Paraguay & Thailand \\
\hline Belarus & El Salvador & Kyrgyzstan & Peru & Togo \\
\hline Belgium & Estonia & Latvia & Philippines & Trinidad \& Tobago \\
\hline Benin & Ethiopia & Lebanon & Poland & Tunisia \\
\hline Bolivia & Finland & Lithuania & Portugal & Turkey \\
\hline \multirow{2}{*}{$\begin{array}{l}\text { Bosnia and } \\
\text { Herzegovina }\end{array}$} & France & Macedonia & Puerto Rico & Uganda \\
\hline & Georgia & Madagascar & Romania & Ukraine \\
\hline Botswana & Germany & Malawi & Russia & United Arab Emirates \\
\hline Brazil & Ghana & Malaysia & Rwanda & United Kingdom \\
\hline Bulgaria & Greece & Mali & Saudi Arabia & Uruguay \\
\hline Burkina Faso & Guatemala & Mauritania & Senegal & USA \\
\hline Burundi & Haiti & Mexico & Serbia & Uzbekistan \\
\hline Cambodia & Honduras & Moldova & Sierra Leone & Venezuela \\
\hline Cameroon & Hungary & Montenegro & Singapore & Vietnam \\
\hline Canada & India & Morocco & Slovakia & Yemen \\
\hline Chad & Indonesia & Mozambique & Slovenia & Zambia \\
\hline Chile & Iran & Myanmar (Burma) & South Africa & Zimbabwe \\
\hline China & Iraq & Nepal & South Korea & \\
\hline
\end{tabular}


The Gallup World Poll uses two primary methodological designs:

- A Random-Digit-Dial (RDD) telephone survey design is used in countries where $80 \%$ or more of the population has landline phones. This situation is typical in the United States, Canada, Western Europe, Japan, Australia, etc.

- In the developing world, including much of Latin America, the former Soviet Union countries, nearly all of Asia, the Middle East, and Africa, an area frame design is used for face-to-face interviewing.

The following are key aspects of the overall Gallup World Poll survey philosophy:

- The sample represents all parts of each country, including all rural areas. Countries are reviewed on a case-by-case basis when part of a country cannot be included in the sample design. The review determines whether the survey should be carried out.

- The target population includes all individuals aged 15 and older.

- Face-to-face interviews are approximately one hour in length. Telephone interviews are considerably shorter, about 30 minutes in length.

- A standard set of questions is used worldwide.

- In the parts of the world where face-to-face surveys are conducted, the questionnaire includes questions tailored to each region. For example, the questions used in heavily indebted poor countries are tailored toward providing information about progress on the Millennium Development Goals.

- The questionnaire is translated into the major languages of each country.

- Interviewing supervisors and interviewers are trained, not only on the questionnaire, but also on the execution of field procedures. This interviewing training usually takes place in a central location.

- Quality control procedures are used to validate that correct samples are selected and that the correct person is randomly selected in each household.

Random respondent selection uses either the latest birthday method or the Kish Grid. The typical World Poll survey in a country consists of 1000 completed questionnaires.

However, in some countries, oversamples may be collected in major cities. For example, we collected an additional 500 interviews in Moscow.

In countries where face-to-face surveys are conducted, census listings of Primary Sampling Units (PSU), consisting of clusters of households, are the main way of selecting the sample. Typically, the PSU are stratified this way: 
I. Cities with population $=1,000,000$ or more

II. Cities with population $=500,000$ to 999,999

III. Cities with population $=100,000$ to 499,999

IV. Cities with population $=50,000$ to 99,999

V. Towns with population $=10,000$ to 49,999

VI. Towns/Rural villages with populations under 10,000

PSU are proportionally allocated to the population in each stratum and typically 125 PSU are sampled with an average of eight interviews, one interview per sampled household, per PSU. If maps of the PSU are available, then they are used; otherwise, the selected PSU must be mapped. Random route procedures are used to select sampled households.

\section{Available Indices Routinely Generated}

In addition to the worldwide gathering of specific questions needed for instantiation of a specific region, indices of beliefs, intentions, and attitudes and perceptions are routinely generated. A full listing is presented below. This broad database could support a wide range of HSBC models and would allow flexibility in our choice of model. 
Table A4-3. Performance indices of the World Path from www.Gallup.com.

\section{Performance Indexes of the World Path}

1. National Leadership Index

This index is an evaluation of national leadership as defined by the confidence people have in key institutions. Institutional components evaluated include the national government, the military, the judicial system, and elections. These items have been a part of Gallup's long history of assessing country-level institutional effectivenses.

2. Law and Order Index

This index represents the security level that citizens observe for themselves and their families. Mcet items in this index emerged from a series of studies on crime and safety that Gallup has conducted in the United States and Europe.

3. Food and Shelter Index

This series of items assesses the capabilitise people have to meet their everyday, basic needs. The items were based on meesures used by the World Bark, USAID, the United Nations, and others.

4. Work Index

Items in this index focus on the degree of personal engagement at work - whether it is paid or a part of day-to-day family subsistence. Many of the items included in this index have emerged from Gallup's expertise in workplace research.

5. Economics Index

The key issues included in this index focus on people's evaluations and outlook for their standard of living and the national economic situation. Galup has consistently investigated this area of "consumer confidence" and related topics.

6. Health Index Personal perceptions of one's own health and incidence of pain, sadness, and wony are measured as a part of this index.

7. Welk-Being Index

Numerous inchiduals, pubications, and groups provided extensive input on this index. Several of the items focus on the works of Cantri, and the later work of Dariel Kahneman and others. Respondents are asked to rate their quality of life now and in the past, and whether they laughed a lot or learned something the day prior to the survey.

8. Engaged Citizens Index

Engaged citiens are positive about the commurities they live in and actively give back to them. Items in this index focus on respondents' satisfaction with their communitise, and their inclination to volunteer their time, money, or assistance to others.
NEW:

9. Emvironment Index

There are several key indicators in this index. Satisfaction with air and water quality is measured along with satisfaction with efforts to deal with erwircnmental issues.

10. Youth Development Index General measures of "development of youth" and "respect for youth" are included in this index along with satisfaction with the educational system.

11. Community Quality of Life Index

This index is a combination of mary sub-indekes covering all aspects of community life. Its purpose is to evaluate a community from the perspective of the pecple who live there. Sub-inderes include:

- Overall satisfaction with community

- Commurity jobs

- Commurity healthcare

- Commurity transportation

- Commurity appearance

- Commurity education

- Commurity leadership

- Commurity housing

- Commurity emvironmental quality

12. Religiosity Index

This index is a meesure of the importance of religion for respondents and their self-reported attendance of religious services. For religions in which attendance at services is limited, care must be used in interpreting the data.

13. Entrepreneurship Index

This index focuses on the intent of respondents in business for themselves and the supportive nature of the community for such a business initiative.

14. Corruption Index

Two items define this indexc respondents' perceptions of comuption in business and perceptions of corruption in govemment.

15. Tolerance Index

This index measures overell perceived cpenness to diversity in respondents' communitise.

16. Communications Index These items measure communications technology available to a respondent. 


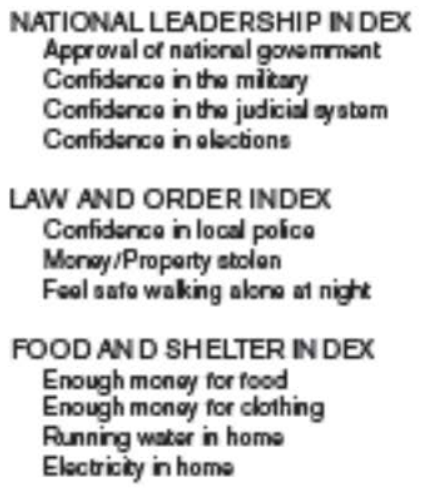

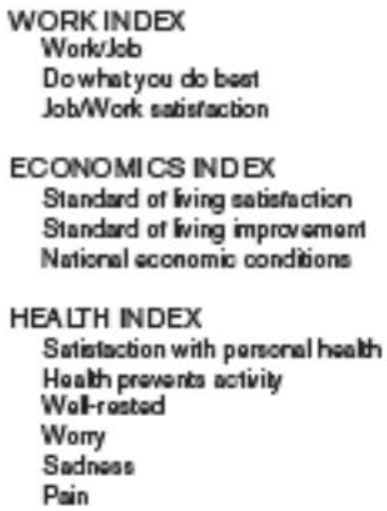

Worry

Sadnese

Pain

WELL-BEING INDEX

Overallire quality - todvy

Overallitre quality - syears from now

Trosked rospectruly

Laughter

Lourn eomething

\section{ENGAGED CITZEN INDEX}

Satiefactionwith community

Perecral freedsms

Generosity

Good place for minoritios

Good place for gywlestiens

\section{OTHER WOR LD POLL INDICATORS}

\section{COMMUNITY QUALTY}

\section{OFUFE NDEX}

Overall satiefaction with community

- Sotiefaction with community

- Pecommend cormunity 10 othere

Community jobs

- Avaiability of good jobs in the community

Community hodthcare

- Access to quality hediheare in tho community

Cormunity transportation

- Public iranepertation in the cormunity

- Poods and highways in the community

Community apposrance

- Besuty of the cormmunity

- Cleaniness of the commurity

Comrunity education

- Community's edxatien rystem
REUGIOSITY IN DEX

Rolgien impertance

Rolgioun eorvice attendence

YOUTH DEVELOPMENT INDEX

Child trostrment

Child loarning opporturity

Schoolveducation sotiefoction

ENTREPRENEURSHIP INDEX

Plan own bueiness

Own busineso

Good place for entreprensure

\section{ENVIRONMENT INDEX}

Ar quality sabisfaction

Water quality sabistacion

Envircnmental gyetom eobisfoction

\section{CORRUPTION INDEX}

Business corruption

\section{TOLERANCE INDEX}

Help stranger

Good place for minortios

Good place for goylestisns

Good place for immigrants

COMMUNICATIONS INDEX

Does your home heve a 1olvision?

Does your home heve a computon?

Do you heve access to the Imiemet? 


\section{Appendix 5 - Dimension Data Quality by Country}

\begin{tabular}{|c|c|c|c|c|c|c|}
\hline Country & Political & Military & Economic & Social & Informational & Infrastructure \\
\hline Andorra & 3 & 0 & 3 & 3 & 3 & 3 \\
\hline $\begin{array}{l}\text { United Arab } \\
\text { Emirates }\end{array}$ & 3 & 3 & 3 & 2 & 3 & 3 \\
\hline Afghanistan & 2 & 3 & 1 & 2 & 2 & 3 \\
\hline Antigua \& Barbuda & 3 & 3 & 3 & 3 & 3 & $\overline{3}$ \\
\hline Anguilla & 3 & 3 & 3 & 3 & 3 & 3 \\
\hline Albania & 2 & 3 & 2 & 2 & 3 & 2 \\
\hline Armenia & 2 & 3 & 2 & 2 & 2 & 2 \\
\hline $\begin{array}{l}\text { Netherlands } \\
\text { Antilles }\end{array}$ & 3 & 3 & 3 & 3 & 3 & 3 \\
\hline Angola & 1 & 1 & 1 & 1 & 1 & 1 \\
\hline Antarctica & 0 & 0 & 0 & 0 & 0 & 0 \\
\hline Argentina & 3 & 3 & 2 & 2 & 2 & 2 \\
\hline American Samoa & 3 & 3 & 3 & 3 & 3 & 3 \\
\hline Austria & 3 & 3 & 3 & 3 & 3 & 3 \\
\hline Australia & 3 & 3 & 3 & 3 & 3 & 3 \\
\hline Aruba & 3 & 0 & 3 & 3 & 3 & 3 \\
\hline Azerbaijan & 1 & 2 & 2 & 1 & 2 & 1 \\
\hline $\begin{array}{l}\text { Bosnia and } \\
\text { Herzegovina }\end{array}$ & 2 & 2 & 2 & 2 & 2 & 1 \\
\hline Barbados & 3 & 3 & 3 & 3 & 3 & 3 \\
\hline Bangladesh & 3 & 2 & 1 & 2 & 2 & 1 \\
\hline Belgium & 3 & 3 & 3 & 3 & 3 & 3 \\
\hline Burkina Faso & 1 & 1 & 1 & 1 & 1 & 1 \\
\hline Bulgaria & 2 & 2 & 2 & 2 & 2 & 2 \\
\hline Bahrain & 2 & 2 & 3 & 2 & 3 & 3 \\
\hline Burundi & 1 & 1 & 1 & 1 & 1 & 1 \\
\hline Benin & 1 & 1 & 1 & 1 & 1 & 1 \\
\hline Bermuda & 3 & 3 & 3 & 3 & 3 & 3 \\
\hline Brunei Darussalam & 3 & 3 & 3 & 3 & 3 & 3 \\
\hline Bolivia & 2 & 2 & 2 & 2 & 2 & 2 \\
\hline Brazil & 2 & 3 & 3 & 2 & 2 & 2 \\
\hline Bahama & 3 & 3 & 3 & 3 & 3 & 3 \\
\hline Bhutan & 2 & 1 & 1 & 1 & 1 & 1 \\
\hline \multicolumn{7}{|l|}{$\begin{array}{l}\text { Burma (no longer } \\
\text { exists) }\end{array}$} \\
\hline Bouvet Island & 1 & 1 & 1 & 1 & 1 & 1 \\
\hline Botswana & 3 & 3 & 3 & 3 & 3 & 3 \\
\hline Belarus & 3 & 3 & 3 & 3 & 3 & 3 \\
\hline Belize & 1 & 1 & 1 & 1 & 1 & 1 \\
\hline Canada & 2 & 2 & 2 & 2 & 2 & 2 \\
\hline $\begin{array}{l}\text { Cocos (Keeling) } \\
\text { Islands }\end{array}$ & 1 & 1 & 1 & 1 & 1 & 1 \\
\hline $\begin{array}{l}\text { Central African } \\
\text { Republic }\end{array}$ & 2 & 1 & 1 & 1 & 1 & 1 \\
\hline
\end{tabular}




\begin{tabular}{|c|c|c|c|c|c|c|}
\hline Country & Political & Military & Economic & Social & Informational & Infrastructure \\
\hline Congo & 1 & 1 & 1 & 1 & 1 & 1 \\
\hline Cook Islands & 3 & 3 & 3 & 3 & 3 & 3 \\
\hline Chile & 3 & 3 & 3 & 3 & 3 & 3 \\
\hline Cameroon & 2 & 2 & 2 & 2 & 2 & 2 \\
\hline China & 3 & 2 & 2 & 2 & 3 & 3 \\
\hline Colombia & 3 & 2 & 3 & 3 & 2 & 3 \\
\hline Costa Rica & 3 & 0 & 3 & 3 & 3 & 3 \\
\hline \multicolumn{7}{|l|}{$\begin{array}{l}\text { Czechoslovakia } \\
\text { (no longer exists) }\end{array}$} \\
\hline Cuba & 1 & 1 & 1 & 1 & 1 & 1 \\
\hline Cape Verde & 3 & 3 & 3 & 3 & 3 & 3 \\
\hline Christmas Island & 0 & 0 & 0 & 0 & 0 & 0 \\
\hline Cyprus & 2 & 2 & 2 & 2 & 2 & 2 \\
\hline Czech Republic & 3 & 3 & 3 & 3 & 3 & 3 \\
\hline \multicolumn{7}{|l|}{$\begin{array}{l}\text { German } \\
\text { Democratic } \\
\text { Republic (no } \\
\text { longer exists) } \\
\end{array}$} \\
\hline Germany & 3 & 3 & 3 & 3 & 3 & 3 \\
\hline Djibouti & 2 & 2 & 2 & 2 & 2 & 2 \\
\hline Denmark & 3 & 3 & 3 & 3 & 3 & 3 \\
\hline Dominica & 3 & 3 & 3 & 3 & 3 & 3 \\
\hline $\begin{array}{l}\text { Dominican } \\
\text { Republic }\end{array}$ & 3 & 2 & 2 & 3 & 3 & 3 \\
\hline Algeria & 3 & 2 & 2 & 2 & 2 & 2 \\
\hline Ecuador & 3 & 2 & 3 & 2 & 2 & 2 \\
\hline Estonia & 3 & 2 & 2 & 2 & 2 & 2 \\
\hline Egypt & 2 & 2 & 2 & 2 & 2 & 2 \\
\hline Western Sahara & 1 & 1 & 1 & 1 & 1 & 1 \\
\hline Eritrea & 1 & 1 & 1 & 1 & 1 & 1 \\
\hline Spain & 3 & 3 & 3 & 3 & 3 & 3 \\
\hline Ethiopia & 1 & 3 & 1 & 1 & 1 & 1 \\
\hline Finland & 3 & 3 & 3 & 3 & 3 & 3 \\
\hline Fiji & 2 & 2 & 2 & 2 & 2 & 2 \\
\hline $\begin{array}{l}\text { Falkland Islands } \\
\text { (Malvinas) }\end{array}$ & 2 & 3 & 3 & 3 & 3 & 3 \\
\hline Micronesia & 2 & 2 & 2 & 2 & 2 & 2 \\
\hline Faroe Islands & 2 & 2 & 2 & 2 & 2 & 2 \\
\hline France & 3 & 3 & 3 & 3 & 3 & 3 \\
\hline $\begin{array}{l}\text { France, } \\
\text { Metropolitan }\end{array}$ & 3 & 3 & 3 & 3 & 3 & 3 \\
\hline Gabon & 1 & 1 & 1 & 1 & 1 & 1 \\
\hline
\end{tabular}




\begin{tabular}{|c|c|c|c|c|c|c|}
\hline Country & Political & Military & Economic & Social & Informational & Infrastructure \\
\hline $\begin{array}{l}\text { United Kingdom } \\
\text { (Great Britain) }\end{array}$ & 3 & 3 & 3 & 3 & 3 & 3 \\
\hline Grenada & 2 & 2 & 2 & 2 & 2 & 2 \\
\hline Georgia & 2 & 2 & 2 & 2 & 2 & 2 \\
\hline French Guiana & 3 & 3 & 3 & 3 & 3 & 3 \\
\hline Ghana & 1 & 1 & 1 & 1 & 1 & 1 \\
\hline Gibraltar & 3 & 3 & 3 & 3 & 3 & 3 \\
\hline Greenland & 3 & 3 & 3 & 3 & 3 & 3 \\
\hline Gambia & 1 & 1 & 1 & 1 & 1 & 1 \\
\hline Guinea & 1 & 1 & 1 & 1 & 1 & 1 \\
\hline Guadeloupe & 2 & 2 & 2 & 2 & 2 & 2 \\
\hline Equatorial Guinea & 1 & 1 & 1 & 1 & 1 & 1 \\
\hline Greece & 3 & 3 & 3 & 3 & 3 & 3 \\
\hline $\begin{array}{l}\text { South Georgia and } \\
\text { the South } \\
\text { Sandwich Islands }\end{array}$ & 2 & 2 & 2 & 2 & 2 & 2 \\
\hline Guatemala & 1 & 1 & 1 & 1 & 1 & 1 \\
\hline Guam & 3 & 3 & 3 & 3 & 3 & 3 \\
\hline Guinea-Bissau & 2 & 2 & 2 & 2 & 2 & 2 \\
\hline Guyana & 1 & 1 & 1 & 1 & 1 & 1 \\
\hline Hong Kong & 3 & 3 & 3 & 3 & 3 & 3 \\
\hline $\begin{array}{l}\text { Heard \& } \\
\text { McDonald Islands }\end{array}$ & 1 & 1 & 1 & 1 & 1 & 1 \\
\hline Honduras & 1 & 2 & 1 & 1 & 1 & 1 \\
\hline Croatia & 1 & 3 & 2 & 2 & 2 & 2 \\
\hline Haiti & 1 & 3 & 2 & 2 & 2 & 2 \\
\hline Hungary & 3 & 3 & 3 & 3 & 3 & 3 \\
\hline Indonesia & 3 & 3 & 3 & 3 & 3 & 3 \\
\hline Ireland & 3 & 3 & 3 & 3 & 3 & 3 \\
\hline Israel & 3 & 3 & 3 & 3 & 3 & 3 \\
\hline India & 3 & 3 & 3 & 3 & 3 & 3 \\
\hline $\begin{array}{l}\text { British Indian } \\
\text { Ocean Territory }\end{array}$ & 2 & 2 & 2 & 2 & 2 & 2 \\
\hline Iraq & 3 & 2 & 2 & 2 & 2 & 2 \\
\hline $\begin{array}{l}\text { Islamic Republic of } \\
\text { Iran }\end{array}$ & 3 & 2 & 2 & 2 & 2 & 2 \\
\hline Iceland & 3 & 3 & 3 & 3 & 3 & 3 \\
\hline Italy & 3 & 3 & 3 & 3 & 3 & 3 \\
\hline Jamaica & 3 & 3 & 3 & 3 & 3 & 3 \\
\hline Jordan & 2 & 2 & 2 & 2 & 2 & 2 \\
\hline Japan & 3 & 3 & 3 & 3 & 3 & 3 \\
\hline Kenya & 2 & 2 & 1 & 1 & 1 & 1 \\
\hline Kyrgyzstan & 2 & 1 & 1 & 1 & 1 & 1 \\
\hline Cambodia & 2 & 2 & 2 & 1 & 1 & 1 \\
\hline Kiribati & 3 & 3 & 3 & 3 & 3 & 3 \\
\hline
\end{tabular}




\begin{tabular}{|c|c|c|c|c|c|c|}
\hline Country & Political & Military & Economic & Social & Informational & Infrastructure \\
\hline Comoros & 2 & 3 & 3 & 3 & 3 & 3 \\
\hline St. Kitts and Nevis & 2 & 1 & 3 & 3 & 3 & 3 \\
\hline $\begin{array}{l}\text { Korea, Democratic } \\
\text { People's Republic } \\
\text { of }\end{array}$ & 3 & 3 & 3 & 3 & 3 & 3 \\
\hline Korea, Republic of & 3 & 3 & 3 & 3 & 3 & 3 \\
\hline Kuwait & 2 & 3 & 3 & 3 & 3 & 3 \\
\hline Cayman Islands & 2 & 2 & 2 & 2 & 2 & 2 \\
\hline Kazakhstan & 2 & 2 & 2 & 2 & 2 & 2 \\
\hline $\begin{array}{l}\text { Lao People's } \\
\text { Democratic } \\
\text { Republic }\end{array}$ & 2 & 2 & 1 & 1 & 1 & 1 \\
\hline Lebanon & 3 & 2 & 1 & 1 & 1 & 1 \\
\hline Saint Lucia & 3 & 3 & 3 & 3 & 3 & 3 \\
\hline Liechtenstein & 3 & 3 & 3 & 3 & 3 & 3 \\
\hline Sri Lanka & 2 & 2 & 2 & 2 & 2 & 2 \\
\hline Liberia & 2 & 1 & 1 & 1 & 1 & 1 \\
\hline Lesotho & 3 & 3 & 3 & 3 & 3 & 3 \\
\hline Lithuania & 3 & 3 & 3 & 3 & 3 & 3 \\
\hline Luxembourg & 3 & 3 & 3 & 3 & 3 & 3 \\
\hline Latvia & 3 & 3 & 3 & 3 & 3 & 3 \\
\hline $\begin{array}{l}\text { Libyan Arab } \\
\text { Jamahiriya }\end{array}$ & 2 & 2 & 2 & 2 & 2 & 2 \\
\hline Morocco & 2 & 2 & 1 & 1 & 1 & 1 \\
\hline Monaco & 3 & 3 & 3 & 3 & 3 & 3 \\
\hline $\begin{array}{l}\text { Moldova, Republic } \\
\text { of }\end{array}$ & 2 & 2 & 2 & 2 & 2 & 2 \\
\hline Madagascar & 2 & 2 & 2 & 2 & 2 & 2 \\
\hline Marshall Islands & 2 & 2 & 2 & 2 & 2 & 2 \\
\hline Mali & 1 & 1 & 1 & 1 & 1 & 1 \\
\hline Mongolia & 3 & 2 & 1 & 1 & 1 & 1 \\
\hline Myanmar & 3 & 3 & 2 & 2 & 2 & 2 \\
\hline Macau & 3 & 3 & 3 & 3 & 3 & 3 \\
\hline $\begin{array}{l}\text { Northern Mariana } \\
\text { Islands }\end{array}$ & 3 & 3 & 3 & 3 & 3 & 3 \\
\hline Martinique & 3 & 3 & 3 & 3 & 3 & 3 \\
\hline Mauritania & 2 & 2 & 2 & 2 & 2 & 2 \\
\hline Monserrat & 2 & 2 & 2 & 2 & 2 & 2 \\
\hline Malta & 2 & 2 & 2 & 2 & 2 & 2 \\
\hline Mauritius & 1 & 1 & 1 & 1 & 1 & 1 \\
\hline Maldives & 1 & 1 & 1 & 1 & 1 & 1 \\
\hline Malawi & 1 & 1 & 1 & 1 & 1 & 1 \\
\hline Mexico & 3 & 3 & 3 & 3 & 3 & 3 \\
\hline Malaysia & 2 & 2 & 2 & 2 & 2 & 2 \\
\hline Mozambique & 1 & 1 & 1 & 1 & 1 & 1 \\
\hline Namibia & 2 & 2 & 2 & 2 & 2 & 2 \\
\hline New Caledonia & 2 & 2 & 2 & 2 & 2 & 2 \\
\hline Niger & 1 & 1 & 1 & 1 & 1 & 1 \\
\hline
\end{tabular}




\begin{tabular}{|c|c|c|c|c|c|c|}
\hline Country & Political & Military & Economic & Social & Informational & Infrastructure \\
\hline Norfolk Island & 3 & 3 & 3 & 3 & 3 & 3 \\
\hline Nigeria & 2 & 2 & 1 & 1 & 1 & 1 \\
\hline Nicaragua & 2 & 3 & 2 & 2 & 2 & 2 \\
\hline Norway & 3 & 3 & 3 & 3 & 3 & 3 \\
\hline Nauru & 3 & 3 & 3 & 3 & 3 & 3 \\
\hline \multicolumn{7}{|l|}{$\begin{array}{l}\text { Neutral Zone (no } \\
\text { longer exists) }\end{array}$} \\
\hline Niue & 1 & 1 & 1 & 1 & 1 & 1 \\
\hline New Zealand & 3 & 3 & 3 & 3 & 3 & 3 \\
\hline Oman & 2 & 2 & 2 & 2 & 2 & 2 \\
\hline Panama & 3 & 3 & 3 & 3 & 3 & 3 \\
\hline Peru & 2 & 2 & 2 & 2 & 2 & 2 \\
\hline French Polynesia & 3 & 3 & 3 & 3 & 3 & 3 \\
\hline $\begin{array}{l}\text { Papua New } \\
\text { Guinea }\end{array}$ & 2 & 2 & 2 & 2 & 2 & 2 \\
\hline Philippines & 3 & 3 & 3 & 3 & 3 & 3 \\
\hline Pakistan & 2 & 2 & 2 & 2 & 2 & 2 \\
\hline Poland & 2 & 2 & 2 & 2 & 2 & 2 \\
\hline $\begin{array}{l}\text { St. Pierre \& } \\
\text { Miquelon }\end{array}$ & 2 & 2 & 2 & 2 & 2 & 2 \\
\hline Pitcairn & 1 & 1 & 1 & 1 & 1 & 1 \\
\hline Puerto Rico & 3 & 3 & 3 & 3 & 3 & 3 \\
\hline Portugal & 3 & 3 & 3 & 3 & 3 & 3 \\
\hline Palau & 3 & 3 & 3 & 3 & 3 & 3 \\
\hline Paraguay & 2 & 2 & 2 & 2 & 2 & 2 \\
\hline Qatar & 2 & 2 & 2 & 2 & 2 & 2 \\
\hline Réunion & 1 & 1 & 1 & 1 & 1 & 1 \\
\hline Romania & 3 & 3 & 3 & 3 & 3 & 3 \\
\hline $\begin{array}{l}\text { Russian } \\
\text { Federation }\end{array}$ & 2 & 2 & 2 & 2 & 2 & 2 \\
\hline Rwanda & 1 & 1 & 1 & 1 & 1 & 1 \\
\hline Saudi Arabia & 2 & 2 & 2 & 2 & 2 & 2 \\
\hline Solomon Islands & 2 & 2 & 2 & 2 & 2 & 2 \\
\hline Seychelles & 2 & 2 & 2 & 2 & 2 & 2 \\
\hline Sudan & 2 & 2 & 2 & 2 & 2 & 2 \\
\hline Sweden & 3 & 3 & 3 & 3 & 3 & 3 \\
\hline Singapore & 3 & 3 & 3 & 3 & 3 & 3 \\
\hline St. Helena & 3 & 1 & 2 & 2 & 2 & 2 \\
\hline Slovenia & 3 & 3 & 3 & 3 & 3 & 3 \\
\hline $\begin{array}{l}\text { Svalbard \& Jan } \\
\text { Mayen Islands } \\
\end{array}$ & 2 & 2 & 2 & 2 & 2 & 2 \\
\hline Slovakia & 3 & 3 & 3 & 3 & 3 & 3 \\
\hline Sierra Leone & 1 & 1 & 1 & 1 & 1 & 1 \\
\hline San Marino & 2 & 2 & 2 & 2 & 2 & 2 \\
\hline Senegal & 1 & 1 & 1 & 1 & 1 & 1 \\
\hline Somalia & 1 & 1 & 1 & 1 & 1 & 1 \\
\hline
\end{tabular}




\begin{tabular}{|c|c|c|c|c|c|c|}
\hline Country & Political & Military & Economic & Social & Informational & Infrastructure \\
\hline Suriname & 1 & 2 & 1 & 1 & 1 & 1 \\
\hline $\begin{array}{l}\text { Sao Tome \& } \\
\text { Principe }\end{array}$ & 2 & 2 & 2 & 2 & 2 & 2 \\
\hline $\begin{array}{l}\text { Union of Soviet } \\
\text { Socialist } \\
\text { Republics (no } \\
\text { longer exists) }\end{array}$ & 0 & 0 & 0 & 0 & 0 & 0 \\
\hline El Salvador & 2 & 2 & 2 & 2 & 2 & 2 \\
\hline $\begin{array}{l}\text { Syrian Arab } \\
\text { Republic } \\
\end{array}$ & 2 & 2 & 2 & 2 & 2 & 2 \\
\hline Swaziland & 3 & 3 & 3 & 3 & 2 & 2 \\
\hline $\begin{array}{l}\text { Turks \& Caicos } \\
\text { Islands }\end{array}$ & 3 & 3 & 3 & 3 & 3 & 3 \\
\hline Chad & 1 & 1 & 1 & 1 & 1 & 1 \\
\hline $\begin{array}{l}\text { French Southern } \\
\text { Territories }\end{array}$ & 1 & 1 & 1 & 1 & 1 & 1 \\
\hline Togo & 1 & 1 & 1 & 1 & 1 & 1 \\
\hline Thailand & 3 & 3 & 3 & 3 & 3 & 3 \\
\hline Tajikistan & 2 & 2 & 2 & 2 & 2 & 2 \\
\hline Tokelau & 1 & 1 & 1 & 1 & 1 & 1 \\
\hline Turkmenistan & 1 & 1 & 1 & 1 & 1 & 1 \\
\hline Tunisia & 2 & 2 & 2 & 2 & 2 & 2 \\
\hline Tonga & 1 & 1 & 1 & 1 & 1 & 1 \\
\hline East Timor & 1 & 1 & 1 & 1 & 1 & 1 \\
\hline Turkey & 3 & 3 & 3 & 3 & 3 & 3 \\
\hline $\begin{array}{l}\text { Trinidad \& } \\
\text { Tobago }\end{array}$ & 1 & 1 & 1 & 1 & 1 & 1 \\
\hline Tuvalu & 1 & 1 & 1 & 1 & 1 & 1 \\
\hline $\begin{array}{l}\text { Taiwan Province } \\
\text { of China }\end{array}$ & 3 & 3 & 3 & 3 & 3 & 3 \\
\hline $\begin{array}{l}\text { Tanzania United } \\
\text { Republic of }\end{array}$ & 1 & 1 & 1 & 1 & 1 & 1 \\
\hline Ukraine & 2 & 2 & 2 & 2 & 2 & 2 \\
\hline Uganda & 1 & 1 & 1 & 1 & 1 & 1 \\
\hline $\begin{array}{l}\text { United States } \\
\text { Minor Outlying } \\
\text { Islands }\end{array}$ & 3 & 3 & 3 & 3 & 3 & 3 \\
\hline $\begin{array}{l}\text { United States of } \\
\text { America }\end{array}$ & 3 & 3 & 3 & 3 & 3 & 3 \\
\hline Uruguay & 2 & 2 & 2 & 2 & 2 & 2 \\
\hline Uzbekistan & 2 & 2 & 1 & 1 & 1 & 1 \\
\hline $\begin{array}{l}\text { Vatican City } \\
\text { State (Holy See) }\end{array}$ & 2 & 2 & 2 & 2 & 2 & 2 \\
\hline
\end{tabular}




\begin{tabular}{|c|c|c|c|c|c|c|}
\hline Country & Political & Military & Economic & Social & Informational & Infrastructure \\
\hline $\begin{array}{l}\text { St. Vincent \& the } \\
\text { Grenadines }\end{array}$ & 2 & 2 & 2 & 2 & 2 & 2 \\
\hline Venezuela & 3 & 3 & 2 & 2 & 2 & 2 \\
\hline $\begin{array}{l}\text { British Virgin } \\
\text { Islands }\end{array}$ & 3 & 3 & 3 & 3 & 3 & 3 \\
\hline $\begin{array}{l}\text { United States } \\
\text { Virgin Islands }\end{array}$ & 3 & 3 & 3 & 3 & 3 & 3 \\
\hline Viet Nam & 2 & 2 & 1 & 1 & 1 & 1 \\
\hline Vanuatu & 1 & 1 & 1 & 1 & 1 & 1 \\
\hline $\begin{array}{l}\text { Wallis \& Futuna } \\
\text { Islands }\end{array}$ & 1 & 1 & 1 & 1 & 1 & 1 \\
\hline Samoa & 3 & 3 & 3 & 3 & 3 & 3 \\
\hline $\begin{array}{l}\text { Democratic } \\
\text { Yemen (no } \\
\text { longer exists) }\end{array}$ & 0 & 0 & 0 & 0 & 0 & 0 \\
\hline Yemen & 2 & 2 & 2 & 1 & 1 & 1 \\
\hline Yugoslavia & 3 & 3 & 3 & 3 & 3 & 3 \\
\hline South Africa & 3 & 3 & 3 & 3 & 3 & 3 \\
\hline Zambia & 1 & 1 & 1 & 1 & 1 & 1 \\
\hline Zaire & 1 & 1 & 1 & 1 & 1 & 1 \\
\hline Zimbabwe & 1 & 1 & 1 & 1 & 1 & 1 \\
\hline
\end{tabular}




\section{Appendix 6 - Real-World Correlation}

The correlation measures the agreement between two data series: the mindshare that is reported by the simulation at each time step (tick), and the dates on which terrorist events occurred (from the WITS database). For a single simulation, the two series might map to a timeline as shown in Figure A6-1, below, in which the red triangles above the timeline are simulated time steps with corresponding mindshare values, and the gray triangles beneath the timeline are WITS incidents.

Figure A6-1. Raw mindshare and WITS incidents

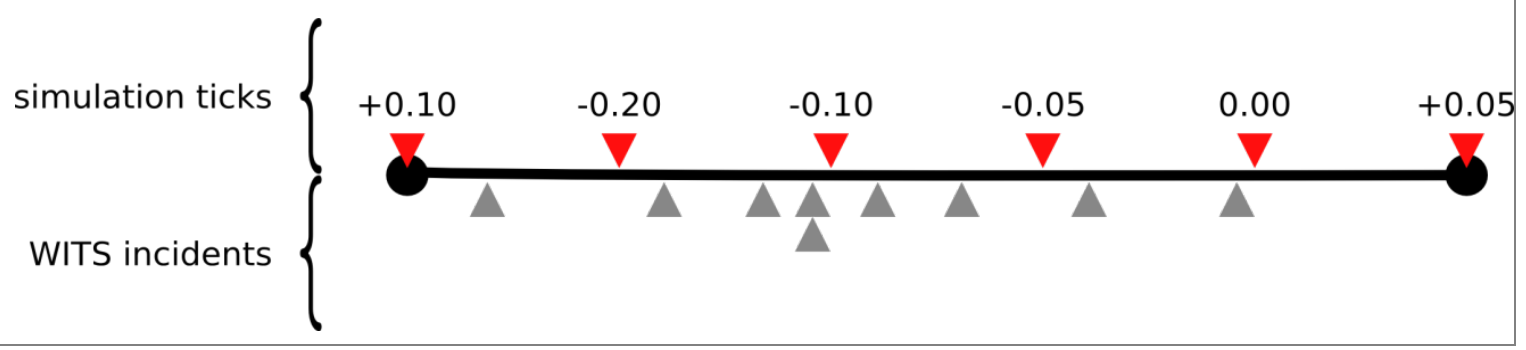

The main challenge to building a correlation is in identifying how best to divide the seven-month (215-day) timeline into regular periods so that it can be sampled consistently across all runs. The data series are both discrete, but the mindshare data represent a sample drawn from a continuously changing quantity, whereas the WITS data are specific incidents that occurred on specific days. Each of these data series is examined separately.

\section{Standardizing Mindshare Data}

Not all of the simulations have the same number of mindshare data points, because the time scale was one of the parameters varied across runs: at 3 days per tick, the seven months elapse in the simulation after only 70 ticks, whereas at 14 days per tick, the same seven-month period includes only 25 ticks.

The simplest way to augment these data points is to rely on linear interpolation, as depicted in Figure A6-2, allowing us to estimate the mindshare values for any day within the 215-day simulation window. The mindshare data are presumed to be nonlinear, as that is one of the key advantages to a CAS simulation, but as each discrete sample represents the smallest increment that the simulation considered, a linear approximation is probably no less reasonable than any other spline; Occam's Razor favors the most direct solution. 
Figure A6-2. Interpolating mindshare

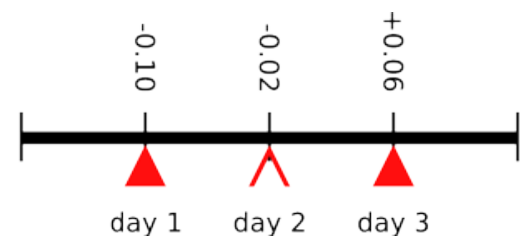

For each of the simulations, linear interpolation will be used to estimate 215 mindshare values, one per day.

\section{Standardizing WITS Data}

The WITS data are gathered from daily observations of terrorist events and inherently include data for all 215 days. Unfortunately, only 60 separate WITS events occurred within this time frame (not all on separate days), so the counts per day are $0,1,2$, or 3 . Even aggregation does not address this problem adequately, as the zeros dominate the data set.

Interpolation and splines are inappropriate, as they presume a continuous variable underlies the data, but the WITS data are discrete. Our approach, as illustrated in Figure A6-3 is to transform each WITS incident from a single-day event into a 5-day event, distributing its 1.0 unit occurrence in a manner that approximates a Gaussian distribution.

Figure A6-3. Distributing a single discrete WITS incident over 5 days

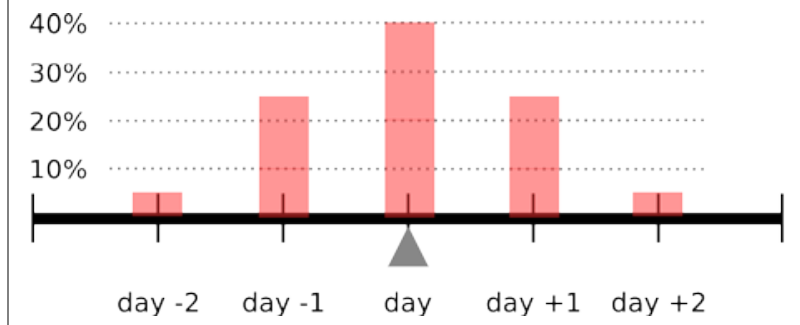

The justification for doing so is that, while the physical act of violence itself has a hard onset, the underlying emotional violence within the populace does not likely ignite instantly but instead waxes and wanes with some period greater than a day. ${ }^{5}$ When these distributions overlap each other, they are summed, as shown in Figure A6-4 below.

\footnotetext{
${ }^{5}$ The CAS can exhibit such flash-point behaviors, but none of our simulations operate on a time scale of as little as one day, so a 5-day cycle may be justified.
} 


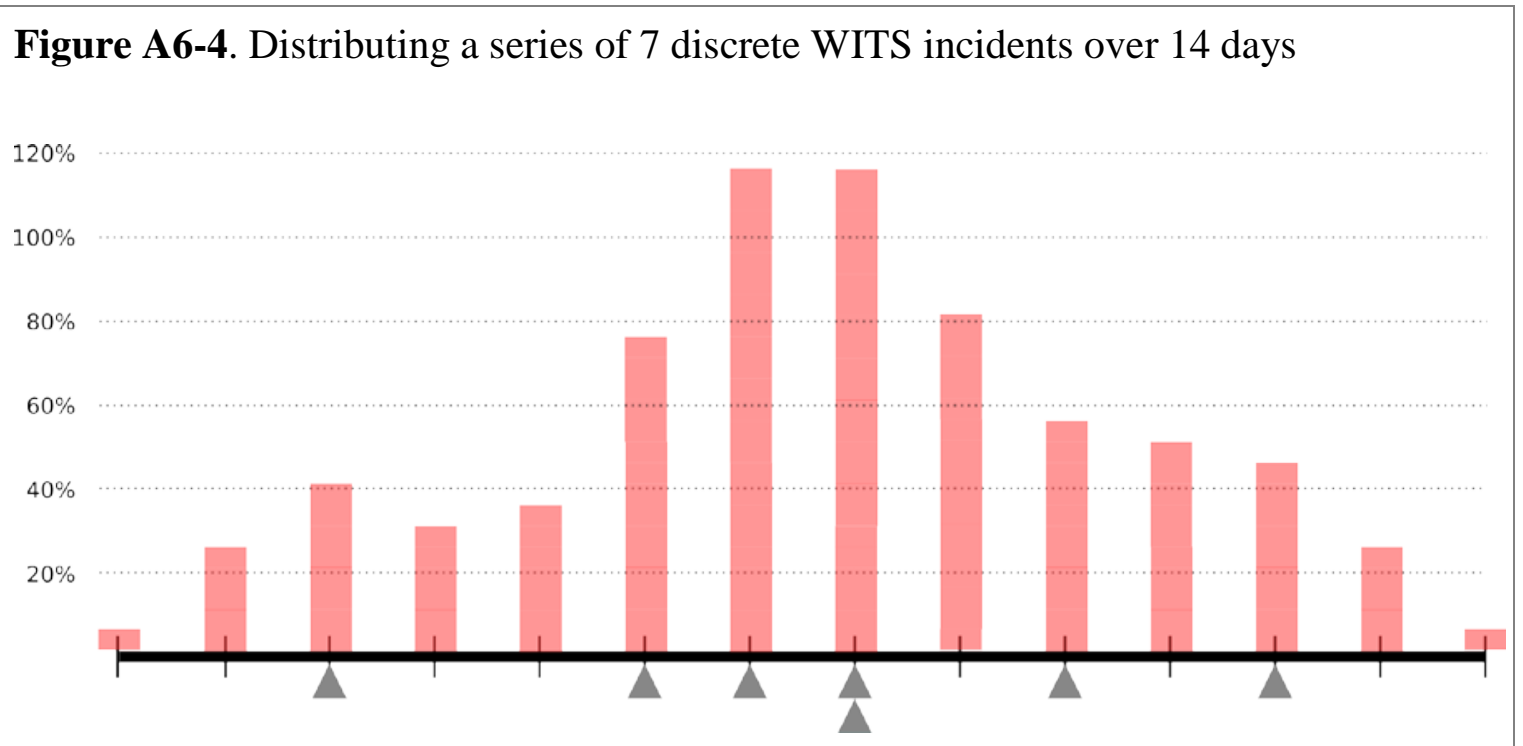

This scheme allows us to smooth the WITS incidents crudely over the period, preserving the trend in the number of incidents per any given subinterval. The total number of WITS incidents across the 215-day period remains 60, but they are distributed more smoothly across the days. The following chart (Figure A6-5) shows the real data from 3/15/2007 to 10/15/2007, both as given (discrete events) and estimated (distributed events).

Figure A6-5. Visualization of distributed WITS data

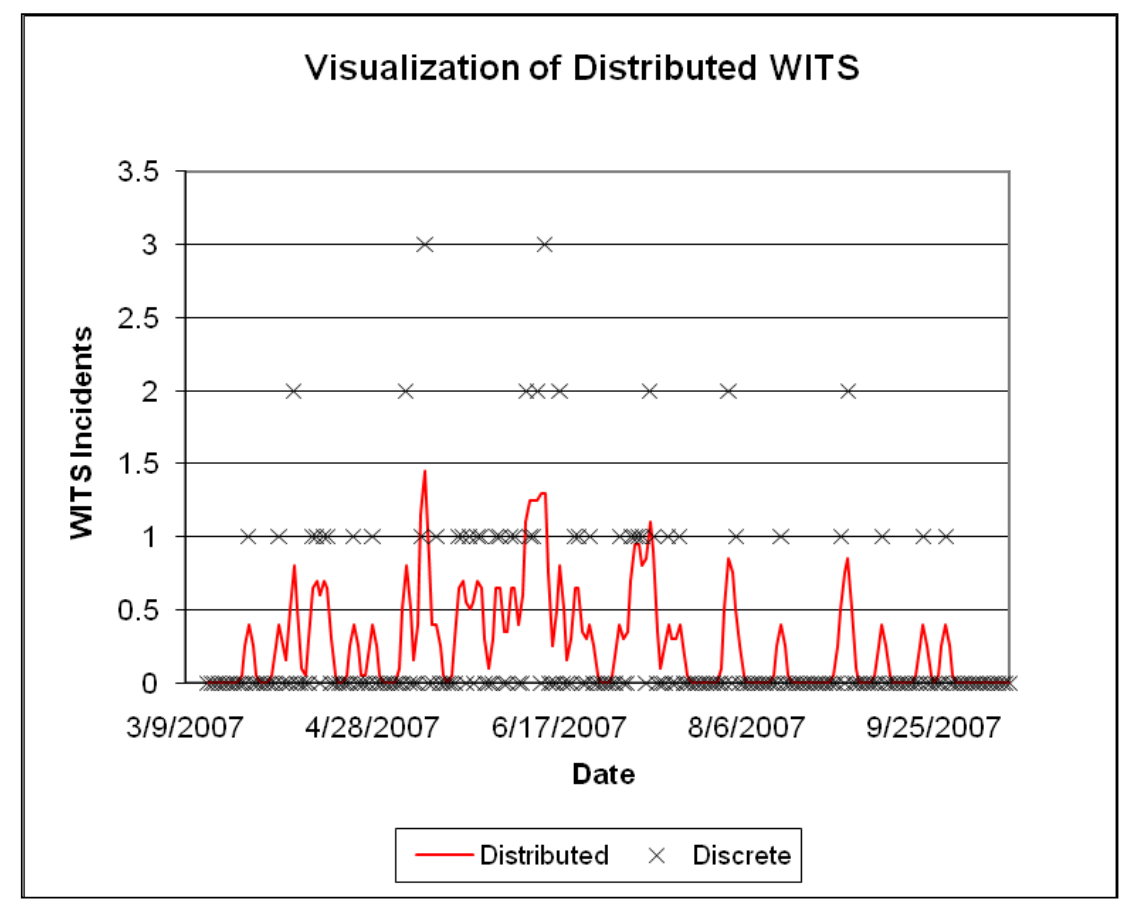




\section{Constructing the Correlation}

Applying the methods discussed in the preceding two sections, we can divide the sevenmonth simulation timeline into as many periods (samples) as we wish to use for constructing the correlation. The question remains: How many periods should we use in the analysis?

We conducted a study across the range of possible periods, from 3 to 100, and observed what happened to the minimum, mean, and maximum correlations between mindshare and WITS incidents across all 180 of the simulations, assuming a 30-day lag between the two data series. The results are reported in Figure A6-6.

Figure A6-6. Correlations with assumed delay

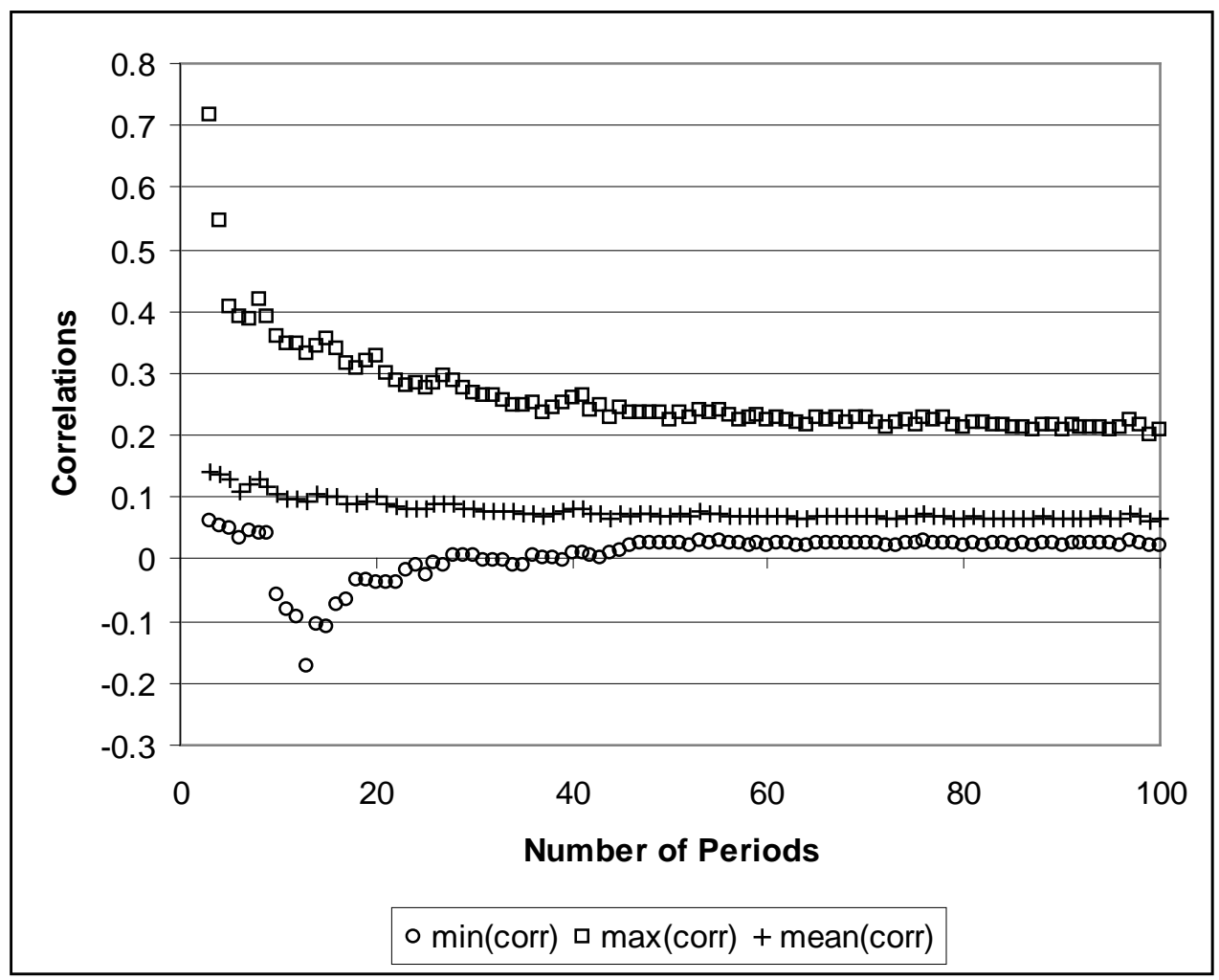

As the 215-day period is divided into more and more periods, the range in correlations asymptotically approaches a constant. 


\section{Appendix 7 - Real-Time Update Code Description}

\section{TimerExample.nlogo}

WHAT IS IT?

Wall-clock is intended as an easy way to pass dates to and from an extension, and to synchronize a simulation with some notion of real time.

This is the "hello world" example for the wall-clock extension. There are four commands and two reporters. The commands are:

set-scale-factor : this command takes the number of milliseconds in a single tick of the simulation. This example uses 86400000 as its scale factor by default, the number of milliseconds in a day.

set-format : this command takes the format you want to use to communicate dates to and from the extension. This is intended to be the easiest possible format, from the simulation programmer's perspective, that java's SimpleDateFormat will accept. For a complete specification of this format, see Java's API

documentation on java.text.SimpleDateFormat.

set-start-time : this command takes the start date of your simulation (the value for tick 0 ), in the format specified above. It's important to set this before the simulation starts running (i.e., at tick $\odot$ )!

set-display-format : this command takes the format you want to use to display dates, typically for "nicely" formatted reporters. This should be a format that is easily readable.

The reporters are:

display-time : reports the current simulation time, in "nice" format.

time : reports the current simulation time, in the default

format.

real-time : reports the wall-clock time, using the

java.util. Calendar class.

SETUP

- - - -

Make sure the wall-clock jar file from the seedling/jar/folder of the project is in the <netlogo install dir>/extensions/wallclock/ folder.

HOW IT WORKS

There isn't really a model in this example, and that isn't really the purpose of this extension! The extension makes use of java.util.Date and java.text.SimpleDateFormatter to provide a simple means for dealing with dates and wall-clock time. The constructor for Date takes the number of milliseconds past Jan. 01, 1970. The extension takes the number of ticks in the 
simulation, multiplies it by the scale factor, and adds the milliseconds after $01-01-70$ of the start date. The result is returned in the format specified by the simulation.

The start time is actually recalculated each time you change the scale factor. See the things to try section for details.

HOW TO USE IT

- - - - - - - -

Initialization:

If you remove the default settings from the procedures, you'll notice the formatters, start date, and scale factor all have default values within the extension. They are:

scale factor $=1000.0$ (one model tick per second)

start date $=$ Jan 01,1970 $00: 00: 00.0 \mathrm{GMT}$, the natural start date for java Dates

format = "MMddyyyy"

display-format = "MMM dd, yyyy hh:mm:ss zzz"

No initialization necessary, you can use the reporters at any time to get wall-clock

values for your simulation.

THINGS TO NOTICE

The monitors show off the reporters doing what they do best, and the inputs allow you to set all the parameters of the extension.

The smallest allowed granularity is milliseconds. This is a restriction of the java Date class and the date format. However, this also means that even if you specify reporting format that's only accurate to the minute, the extension will always keep track of it to the millisecond precision.

THINGS TO TRY

Click go to watch it step through a day at a time, or click goforever and experiment with the scale-factor slider.

Run the experiment for a long time with a large scale factor, then slow time way down to watch how the extension calculates the new time. Notice the extension behaves as you would expect, time slows down. The extension doesn't continue to calculate time based on the offset from the simulation's start time, which would cause a jump backwards in time if the scale factor was set to something much smaller mid-simulation.

EXTENDING THE MODEL

This could be extended to any simulation that needs a real-time component. 


\section{GeocodeExample.nlogo}

WHAT IS IT?

This example demonstrates the geocode functionality as a standalone netlogo extension. In the seedling/jar folder is another jar, fullsimframe.jar, that has all the geocoding, wall-clock, and simframe functionality bundled in one extension.

ìTalibanî is a general model that allows users to explore interactions and consequences among the diverse forces currently in Afghanistan. This example added the geocode functionality to the Taliban model, to demonstrate its functionality on a pertinent map. Two additional buttons have been added to the original model: "Kabul" and "Kandahar." Each button highlights the patch corresponding to each city.

SETUP

- - - -

Make sure the geocode.jar from seedling/jar is in the <netlogo install>/extensions/geocode/ folder.

HOW IT WORKS

The geocode extension uses the Google Map API to request lat-long coordinates. The extension caches these results, reporting them to the model as either patch coordinates (based on the bounds of the world), or lat-long coordinates, reported in decimal degrees.

HOW TO USE IT

open a browser to maps.google.com. Google maps accepts all forms of unstructured queries, and any query that returns a valid result on the website will also return a geocode with the extension. For the two examples provided here, "Kabul" was a valid query. However, for Kandahar, the query "Kandahar, Afghanistan" was required to receive a result.

In order to use the google maps api, you must apply for a key at http://code.google.com/apis/maps/signup.html. The key provided in the example SHOULD NOT be reused.

This key, along with the bounds of the map, are passed in to the extension's initialization procedure. The map bounds in terms of patch coordinates are retrieved automatically when the initialization procedure is called. Currently, the geocode extension only supports box worlds.

For this example, the following initialization was used: geocode:init 29.40611 60.50417 38.47198 74.91574 "ABQIAAAAmd7V71yw9ZddA๑s8Z3wSKBQ0Y93X9JJIv4oRUIhVDwundpXiNhTCZmoj XGlyeRZ7KKGgGRE@GgKFpQ"

The four numbers are the latitude and longitude of the top and bottom corners. These values were retrieved from the "Afghanistan" article on Wikipedia. The string is the Google key. 
The extension comes with four reporters, xpcor, ypcor, lat, and lon. All these reporters take in a string query, and return a decimal vaue. Xpcor and Ypcor provide the patch coordinates that can be passed directly to ask patch, as in the show-city procedure in this example. Lat and lon return decimal degree values.

THINGS TO NOTICE

-.-.-.-.-.-.-.

Google's geocoding isn't perfect. Kandahar is actually an example of the inaccuracies that it can occasionally produce. If you navigate to maps.google.com and zoom in on

Afghanistan, you'll see Kandahar labeled on the map. If you then actually * search* for

Kandahar, the search result points to an area about 50 miles

Southwest of where the map says Kandahar is. It's the *search result location, * not the actual labeled location, that would be returned by this geocoding extension. 


\section{RealtimeTest.nlogo}

WHAT IS IT?

This model shows how to use the simframe extension, bundled with the wall-clock functionality, to deliver events to a running simulation.

This example only shows invocation of simulation functions. The simframe2 model shows how to use the reaction grammar to add extensions to simframe in the form of additional jar files.

SETUP

Make sure that "events.txt" and "test.txt" are in the same folder as this model. Also, make sure seedling/jar/simframe.jar is in $<$ netlogo install>/extensions/simframe/.

HOW IT WORKS

Simframe uses two initialization files, a reaction file (test.txt) and an events file (events.txt). The reaction file specifies all the information necessary to take events as they arrive and use them to update the running simulation at the appropriate time.

Let's examine the reaction file line-by-line:

event-attributes specifies the names of the different attributes an event will have. They can be referred to as parameters passed to functions, and also to select which event handler to use for a given event. The line must begin with "event-attributes"

followed by a white-space-delimited list of attribute names.

The next line specifies the type of each attribute in the same order. Possible values are numeric, string, and time.

The next section of the reaction file specifies transforms. In netlogo, these correspond to reporters, or in general, functions that return a value. These values can then be passed as parameters to other transforms. If a transform returns a set of agents or patches, other functions can be called *on* these returned values. For instance, the line "sim.die() on sim.blueturtles()" would call the die function on the agents that the blue-turtles transform returns. A transform declaration should be of the form:

transform return-type name ( param-types)

Return-type can be numeric, string, time, agent, agents, location, or locations. The name should start with "sim." or "realtime." denoting whether it is a simulation function or an extension function written in java that simframe should invoke directly. The param-types can be numeric, string, or time, and should appear in a comma-delimited list.

The next section of the file includes event handlers. They are declared as follows: 


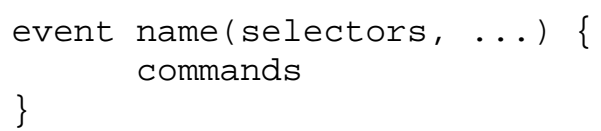

The name must be unique. Selectors are the values of the event attributes that trigger this particular event handler. They can either be specific values, or "*" for any value. For instance, in the sample text file, the event handler red_run will be triggered for every event with id $=1$ and any headline.

Commands are the procedures which are called when this event is triggered. The line starts with a function to call, either beginning with "sim." or "realtime." Parameters can be literal values, or event attributes. For example, if the event id were to be passed to a particular command, it would be referenced as "event.id":

sim.left(event.id) on sim.red-turtles()

Optionally, a transform that specifies a particular agent or location set can be added to the end of the command, using the keyword "on." For sim commands, use the function name exactly as it would appear in netlogo (left, forward, separate, red-turtles, and blue-turtles, are all functions defined in the nlogo file). For realtime commands, use the fully-qualified class name, followed by the method name (for an example of this see the simframe2 model).

The events file is simply a text file full of events. The first token is the date, specified in the format given in the nlogo file (using the wall-clock functionality). The attributes of the event itself are speicied inside the parenthesis, as a commadelimited list of literals (strings, numerics, or times).

HOW TO USE IT

Click setup, then go-forever, and watch as the turtles react to the events as they arrive at different times.

The "test" button shows a simple example of how simframe works: during each tick, the simulation receives a string of code from simframe to interpret and run. Test-code simply returns the string "separate" from simframe, and then netlogo runs it. 


\section{RealtimeExample2.nlogo}

WHAT IS IT?

This model shows how to use the simframe extension, bundled with the wall-clock functionality, to deliver events to a running simulation.

This example shows invocation of simulation and realtime functions. The simframe 2 model shows how to use the reaction grammar to add extensions to simframe in the form of additional jar files.

SETUP

- - - -

Make sure that "events.txt" and "test.txt" are in the same folder as this model. Also, make sure seedling/jar/simframe.jar and seedling/jar/mapmgr.jar are in <netlogo

install>/extensions/simframe/.

HOW IT WORKS

-......... - -

Simframe uses two initialization files, a reaction file (test.txt) and an events file (events.txt). The reaction file specifies all the information necessary to take events as they arrive and use them to update the running simulation at the appropriate time.

Let's examine the reaction file line-by-line:

event-attributes specifies the names of the different attributes an event will have. They can be referred to as parameters passed to functions, and also to select which event handler to use for a given event. The line must begin with "event-attributes"

followed by a white-space-delimited list of attribute names.

The next line specifies the type of each attribute in the same order. Possible values are numeric, string, and time.

The next section of the reaction file specifies transforms. In netlogo, these correspond to reporters, or in general, functions that return a value. These values can then be passed as parameters to other transforms. If a transform returns a set of agents or patches, other functions can be called *on* these returned values. For instance, the line "sim.die() on sim.blueturtles()" would call the die function on the agents that the blue-turtles transform returns. A transform declaration should be of the form:

transform return-type name ( param-types)

Return-type can be numeric, string, time, agent, agents, location, or locations. The name should start with "sim." or "realtime." denoting whether it is a simulation function or an extension function written in java that simframe should invoke directly. The param-types can be numeric, string, or time, and should appear in a comma-delimited list. 
The next section of the file includes event handlers. They are declared as follows:

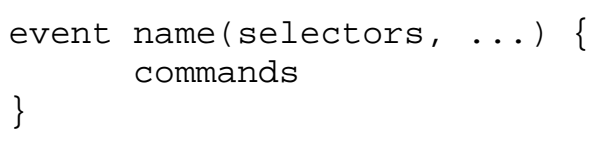

The name must be unique. Selectors are the values of the event attributes that trigger this particular event handler. They can either be specific values, or "*" for any value. For instance, in the sample text file, the event handler red_run will be triggered for every every with $i d=1$ and any headline.

Commands are the procedures which are called when this event is triggered. The line starts with a function to call, either beginning with "sim." or "realtime." Parameters can be literal values, or event attributes. For example, if the event location were to be passed to a particular command, it would be referenced as "event.location":

realtime.geocode.MapManager.geoCodeX ( event.location )

Optionally, a transform that specifies a particular agent or location set can be added to the end of the command, using the keyword "on." For sim commands, use the function name exactly as it would appear in netlogo (left, forward, separate, red-turtles, and blue-turtles, are all functions defined in the nlogo file). For realtime commands, use the fully-qualified class name, followed by the method name:

realtime.realtimeext.MapManagerExt.init ( $\odot . \odot, 0 . \odot, 40 . \odot, 40 . \odot$, 29.40611, 60.50417, 38.47198, 74.91574, "googlekey" )

The events file is simply a text file full of events. The first token is the date, specified in the format given in the nlogo file (using the wall-clock functionality). The attributes of the event itself are speicied inside the parenthesis, as a commadelimited list of literals (strings, numerics, or times).

\section{EXTENSIONS TO SIMFRAME}

This example shows a method for adding extensions to simframe in the form of external jar files. This particular example exposes the geocode functionality as an extension to simframe rather than as its own extensions to netlogo. Realistically it makes more sense for geocoder to be a netlogo extension, it was merely used in this way for demonstrative purposes. Ideally, simframe should be used as a bundle with both the wall-clock and geocode funtionality. To do this, delete the simframe and mapmgr jars from the <netlogo install>/extensions/simframe/, rename fullsimframe.jar to simframe.jar, and copy it to <netlogo install>/extensions/simframe/.

HOW TO USE IT

Click load, and then the step button ">" twice, and watch as the two cities passed in from the events are highlighted. 


\section{RealtimeExample3.nlogo}

WHAT IS IT?

This model shows how to use the simframe extension, bundled with the wall-clock functionality, to deliver events to a running simulation.

This example shows invocation of simulation and realtime functions. The simframe3 model extends simframe2 to show how to receive events from distributed services, using SOAP requests.

SETUP

- -

Make sure that "events.txt" and "test.txt" are in the same folder as this model. Also, make sure seedling/jar/simframe.jar and seedling/jar/mapmgr.jar are in <netlogo

install>/extensions/simframe/. Also, 4 libs from the seedling/lib folder need to be in the <netlogo

install>/extensions/simframe/ folder:

-xerces.jar

-activation.jar

-mail.jar

- soap.jar

To set up the SOAP service, the remote machine needs to have some servlet container (I used Apache Tomcat). Copy the soap.war file from seedling/jar to the webapps folder of your servlet container. Also, copy the events.txt file from the simframe2 example to the bin/ folder of the servlet container (this is the default root path, and the remote service will read this file in and provide those events to the model).

To deploy the service, the easiest way is from the command line:

java org.apache.soap.server.ServiceManagerClient

http://localhost:8080/soap/servlet/rpcrouter deploy soap.xml

The classpath will need to point to the four jars listed above for this java command to work properly.

HOW IT WORKS

- - - - - - - - -

Simframe uses two initialization files, a reaction file (test.txt) and $a{ }^{*}$ local* events file (events.txt), which can be empty in this case since we're pulling events from a remote source. The reaction file specifies all the information necessary to take events as they arrive and use them to update the running simulation at the appropriate time.

Let's examine the reaction file line-by-line:

event-attributes specifies the names of the different attributes an event will have. They can be referred to as parameters passed to functions, and also to select which event handler to use for a given event. The line must begin with "event-attributes"

followed by a white-space-delimited list of attribute names. 
The next line specifies the type of each attribute in the same order. Possible values are numeric, string, and time.

This reaction file has a new declaration, a CHANNEL. Channels take a url and an id. In the example provided, the remote service is actually being hosted locally, so for a true remote test this url would need to point elsewhere.

The next section of the reaction file specifies transforms. In netlogo, these correspond to reporters, or in general, functions that return a value. These values can then be passed as parameters to other transforms. If a transform returns a set of agents or patches, other functions can be called *on* these returned values. For instance, the line "sim.die() on sim.blueturtles()" would call the die function on the agents that the blue-turtles transform returns. A transform declaration should be of the form:

transform return-type name ( param-types )

Return-type can be numeric, string, time, agent, agents, location, or locations. The name should start with "sim." or "realtime." denoting whether it is a simulation function or an extension function written in java that simframe should invoke directly. The param-types can be numeric, string, or time, and should appear in a comma-delimited list.

The next section of the file includes event handlers. They are declared as follows:

event name(selectors, ...) \{ commands \}

The name must be unique. Selectors are the values of the event attributes that trigger this particular event handler. They can either be specific values, or "*" for any value. For instance, in the sample text file, the event handler red_run will be triggered for every every with $i d=1$ and any headline.

Commands are the procedures which are called when this event is triggered. The line starts with a function to call, either beginning with "sim." or "realtime." Parameters can be literal values, or event attributes. For example, if the event location were to be passed to a particular command, it would be referenced as "event.location":

realtime.geocode.MapManager.geoCodeX ( event.location )

Optionally, a transform that specifies a particular agent or location set can be added to the end of the command, using the keyword "on." For sim commands, use the function name exactly as it would appear in netlogo (left, forward, separate, red-turtles, and blue-turtles, are all functions defined in the nlogo file). For realtime commands, use the fully-qualified class name, followed by the method name: 
realtime.realtimeext.MapManagerExt.init ( $\odot . \odot, 0 . \odot, 40 . \odot, 40 . \odot$, 29.40611, 60.50417, 38.47198, 74.91574, "googlekey" )

The events file is simply a text file full of events. The first token is the date, specified in the format given in the nlogo file (using the wall-clock functionality). The attributes of the event itself are speicied inside the parenthesis, as a commadelimited list of literals (strings, numerics, or times).

\section{EXTENSIONS TO SIMFRAME}

This example shows a method for adding extensions to simframe in the form of external jar files. This particular example exposes the geocode functionality as an extension to simframe rather than as its own extensions to netlogo. Realistically it makes more sense for geocoder to be a netlogo extension, it was merely used in this way for demonstrative purposes. Ideally, simframe should be used as a bundle with both the wall-clock and geocode funtionality. To do this, delete the simframe and mapmgr jars from the <netlogo install>/extensions/simframe/, rename fullsimframe.jar to simframe.jar, and copy it to <netlogo install>/extensions/simframe/.

HOW TO USE IT

Click load, and then the step button ">" several times, and watch as the three cities passed in from the events are highlighted, two remotely and one locally. 


\section{Appendix 8 - Generating Narrative}

The relatively simple simulations that have been generated as part of this seedling effort still generate rather large volumes of data. Approximately 10 data values could be reported for each of the few thousand agents (and for each of the $~ 800$ patches) per time step, with 50 or more time steps in short runs. We have been tracking only cursory data elements from these runs, and storing them in a common database to facilitate the data entry, but with well over 2000 runs in the collection, the total amount of data has been manageable only through SQL queries and human analyst intervention.

The challenge clearly is how best to communicate what has happened and what is important to casual users of the system. Commanders in the field, for example, cannot be expected to troll through millions of rows of data describing a simulation, but instead wish to know what interventions are most likely to have the effects they seek, and to be informed of the most-often encountered side effects. Satisfying these requirements is an exceptionally difficult task (Kittredge 2000) and will require dedicated study. This document attempts to summarize some of the issues that are required to address the problem.

Fundamentally, this is the problem of reducing voluminous quantitative data to parsimonious qualitative data. There appear to be three general themes:

1. framing the task

2. the mechanics of solution

3. the art of solution

Each of these will be reviewed in a subsequent section. We also connect each of these three themes to our Afghanistan model using a subset of our experimental runs as an example. This subset concerns the evaluation of multiple COAs, as taken from Task 4: Section III - Evaluating Command Options for Ghazni in 2006.

\section{Framing the Task}

Qualitative data are important in their own right (Van Maanen 1979). And yet, multiple layers are implicit in the construction of any narrative (Pentland 1999), with each layer having an independent context that matters (Miles 1979). Qualitative data represent a significant form of data reduction, eliding the details that do not pertain to the core message, and highlighting the most important elements of the idea being expressed. And yet without the appropriate context, these compressed ideas become increasingly misleading.

In this seedling, we have tried to demonstrate how a generic, workbench-type framework can be developed to support multiple social theories and multiple engagement types, but the aggregation and reporting of simulation results to a field commander require an 
implicit understanding of the analysis task to be performed. We have to know what questions are being asked, so that we know how best to sieve through the simulation details to summarize what has occurred (Kukich 1983).

In one of our experimental sets of simulation runs, we evaluated what would happen in Ghazni during 2006 given six different combinations of economic incentives and troop surge levels. This is an example of the type of information a field commander would find useful when evaluating various options. It also highlights some of the pitfalls that could occur if the simulation results are not properly framed within the context of key assumptions made by the model or the experimental design.

For example, we consider three different surge levels, but without defining what form such a surge would take. Does the surge represent a single large base of operations or multiple forward-operating bases (FOBs)? Are the troops patrolling as a well-defended force or in smaller groups that have more direct interaction with the local population? Presumably these details are known in the calibration and validation of the Afghanistan model, but such issues must be properly communicated when presenting the results of the experiments so the commander will have a greater degree of confidence in the model outputs and a framework for understanding their significance.

\section{The Mechanics of Solution}

The specific mechanics of solution vary across proposals. Some systems use prestructured grammars to map event data patterns to plain-text expressions (Pentland 1995). Others use human-defined templates to aggregate event-level data (Kumar 2007). All of these solutions concern how best to reduce the quantity and specificity of the data to the smaller domain of the human problem being summarized in natural language ( $\mathrm{Yu}$ 2006, Maybury 1995). The key problems are that while replication may boost confidence in the results (Eisenhardt 1989, Jick 1979), even simple aggregation methods such as distance functions for binning can be woefully inconsistent (Sabherwal 1993).

The results of the experiment referred to above would require any such templates or grammars to be very domain-and even model-specific. The visual-analytic tools that are described in Appendix 9 go a long way in addressing some of the issues with aggregating the outputs of various experiments in real-time, as well as allowing more direct and intuitive control of simulation runs as they are progressing. Nevertheless, additional tools and templates would be needed to, for example, perform statistical analysis across multiple, repeated experiments. Currently in our experiments, these types of aggregation and analysis are mainly performed using external databases, spreadsheets, and statistical tools, which are then summarized by expert operators of the model.

\section{The Art of Solution}

As would be expected of any system producing output for non-expert users, the form of the presentation matters. This means that simple issues (for a human writer) such as word choice can have substantial influence on the quality of the outcome (Reiter 2005). 
Though we think of the machines and software as cold and antiseptic, the rhetoric we use in framing the map from data to natural language also can have a substantial impact on the message the end-user takes away (Golden-Biddle 1993).

Such a presentation would necessarily include a translation or mapping from the simulation data to real-world situational parameters. For example, it is not enough to say that $400 \mathrm{CF}$ agents and pro-government economic incentives are needed to achieve favorable mindshare for the Ghazni region. The definition of a synthetic agent in terms of how many soldiers (and what type of soldiers) it represents must be incorporated to articulate a meaningful COA. Also, a description of the costs, the assumptions, and the detailed characteristics of these COAs would have to be included so that the commander can fully understand the issues and tradeoffs involved in choosing among multiple options suggested by the simulation experiments. Presumably, these mappings would be an inherent aspect of a rigorously designed and validated model. Still, integrating these parameters into a useful presentation of experimental results is a necessary component of generating a natural language narrative for the nonexpert end user. 


\section{Appendix 9 - Visual Analytics}

Our agent-based simulation allows users to visualize the results of experimenting with different social theories and scenarios in Afghanistan. Here we see the original NetLogo interface (A9-1). Notice the large portion of screen-space dedicated to sliders and other control elements.

Figure A9-1. Original NetLogo home page

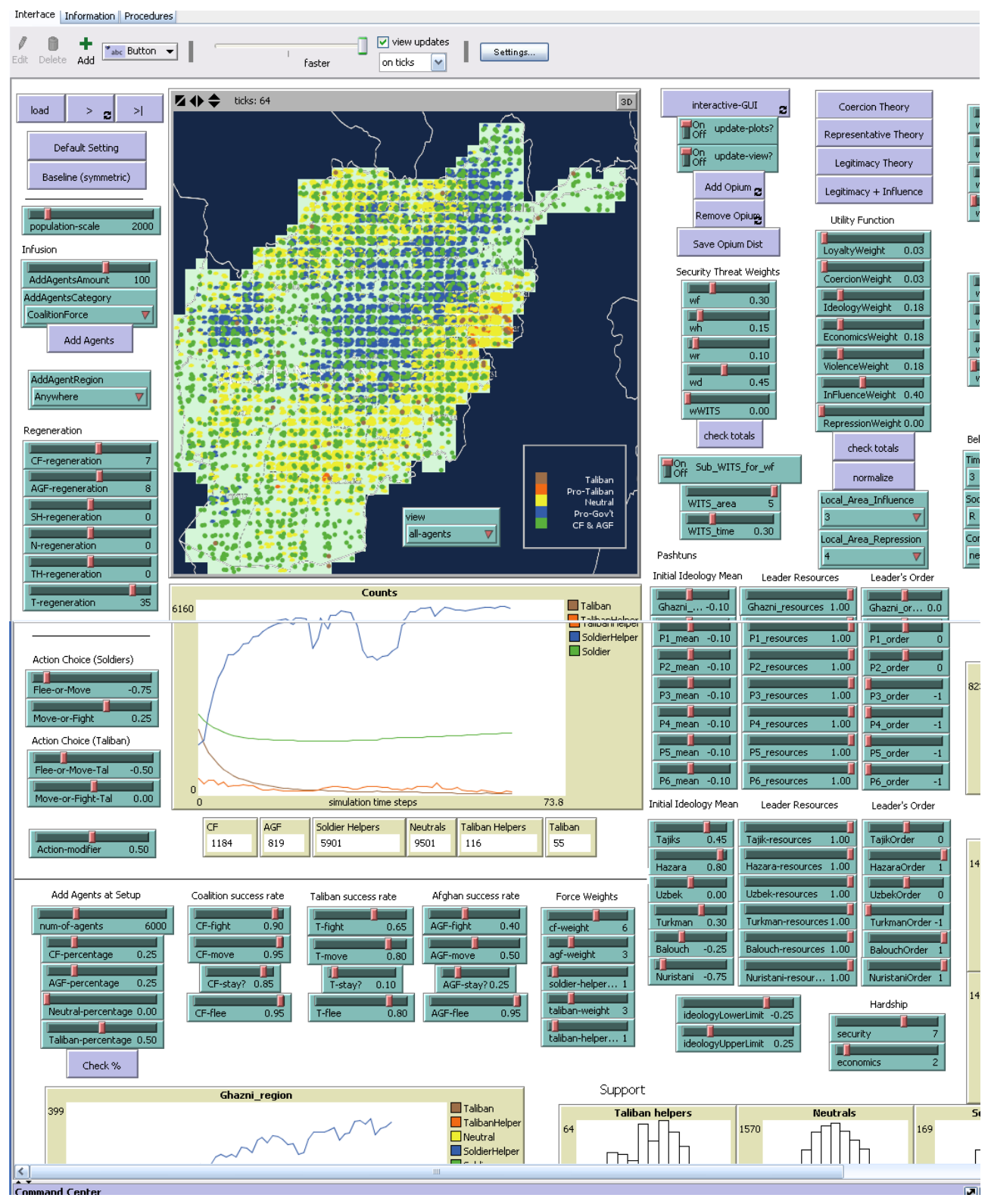


NetLogo is limited to a single perspective that is tied to the viewable map area. Similarly, the additional infovis views in the agent-based tool, such as the bar chart and the timeseries view, are also tied to this single perspective.

The main purpose of this agent-based tool is to allow the user to manipulate variables within the simulation and visualize the effects of the changes. Some of these variables are global in that they affect the simulation of the entire country, while others are tied to fixed single locations or to specific regions. It is clear that without proper organization, an exponential number of controls is needed to capture all combinations of all the variables. In fact, Figure A9-1 shows many of the 200 sliders that are needed to operate the original NetLogo model.

To facilitate control and analysis of the Afghanistan model, we apply the "probe" concept to the existing visualization of the NetLogo agent-based system. We define a probe as a pair consisting of a user-defined region-of-interest and a pane containing any variety of information visualizations coordinated to depict and interact with the data within that region-of-interest. The region-of-interest and the visualization pane are linked either directly (e.g., by a line) or indirectly (e.g., the region-of-interest and the pane's background are shaded the same color).

To create a probe, the user selects a region-of-interest (e.g., a central focal point and extent radius, or a manually selected irregularly shaped region) and then chooses a location for the visualization pane to be overlaid directly within the main geospatial visualization. Once created, a probe visually presents a focused, local view into the dataset/model along with an intuitive visual linkage back to the overall dataset/model.

The introduction of probes almost completely transformed the NetLogo tool (Figure A92). 
Figure A9-2. An example workspace in our new, probe-based interface. Notice that the user can add any number of different overview maps. Probes can then be inserted into these maps, spawning linked coordinated visualization/interaction panes. This extends observation and interaction across all levels, from global to individual cells.

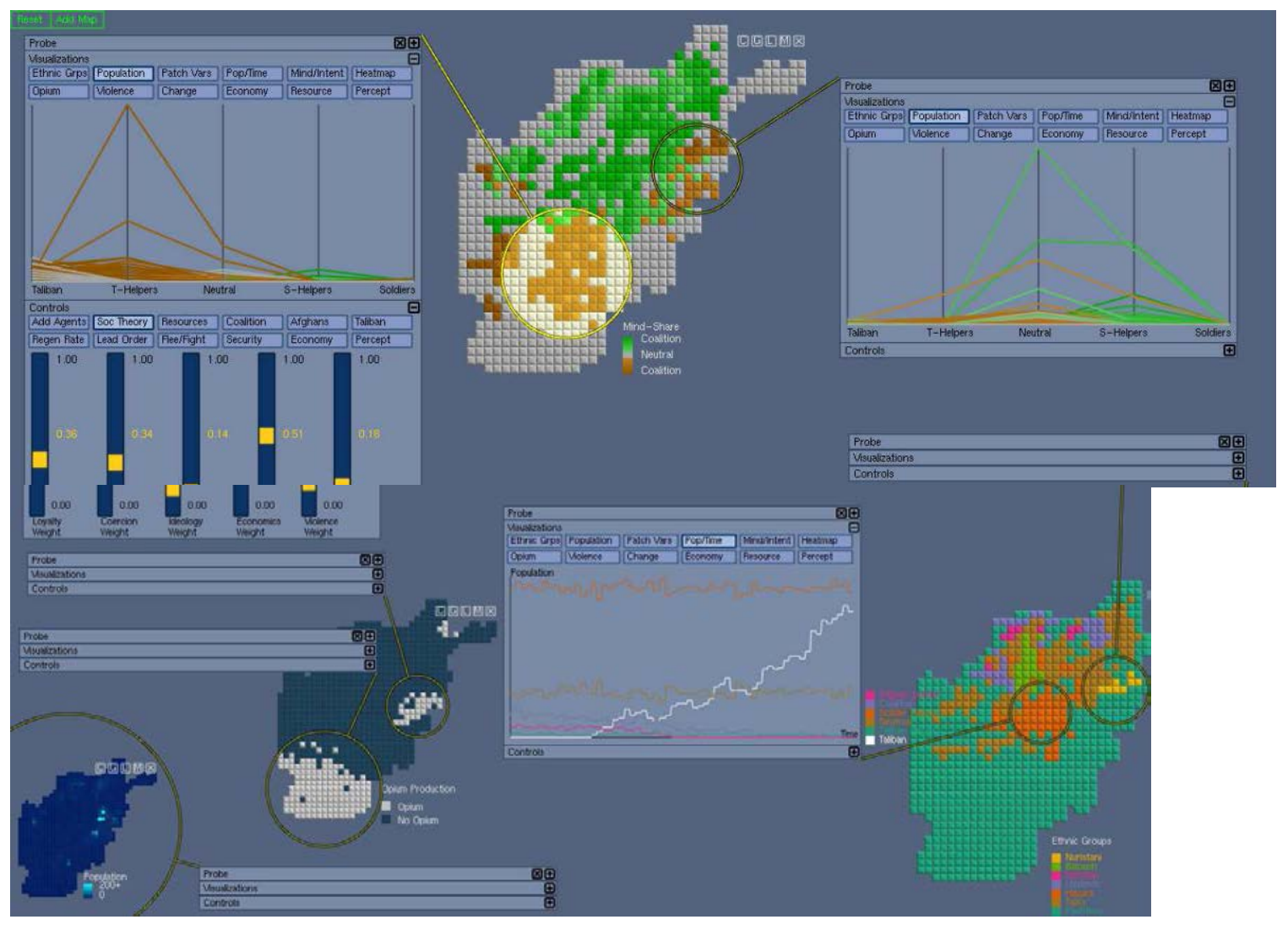

In addition to the issue of overcrowding from excessive sliders (Figure A9-1), the agentbased tool suffers another equally severe interaction issue, in that the sliders offer no spatial context in terms of their relationships to the corresponding geographical regions. Users and observers of this agent-based simulation are often left wondering which slider to operate to affect a specific region-of-interest. This incongruity between the visualization and its controls greatly diminishes the effectiveness of the simulation as an experimental platform for testing social theories.

By applying our probe concept, we can greatly increase the effectiveness of the interface. As can be seen in Figure A9-2, multiple maps are now allowed, with each map colored based on a particular dimension in the data (e.g., ethnic group, loyalty, etc.). Most importantly, the 200 sliders can now be replaced by an "on-demand” tabbed control panel of sliders directly associated with each probe (Figure A9-3). This combination of sliders with geolocated probes makes the effect of each slider intuitive and obvious, in that interaction with a slider now affects only the region tied to its corresponding probe. 
The implication of a visualization that has capabilities for both passive inspection and active manipulation is striking. As shown in Figure A9-3, the user has selected two nested regions to test the impact of an increased economy in a small selected region and its effect in the surrounding areas. With the probe interfaces, the user can directly modify the economy of the small selected region and observe its effects in the probe associated with the surrounding areas.

Figure A9-3. The use of localized control capabilities is shown in this scenario. Here, the user places a probe (the smaller circle) over the city of Kabul and expands the control section of that probe's interface and manipulates local variables to test out a new social theory within the city limits. A second probe (the larger circle) has been added to encompass the surrounding region, which has some pockets of Taliban loyalists (brown cells). This probe is set up to graph the relative populations of various factions over time. The user can easily see that after the new social theory is enacted within Kabul, the number of Taliban agents (white line) in the surrounding areas decreases.

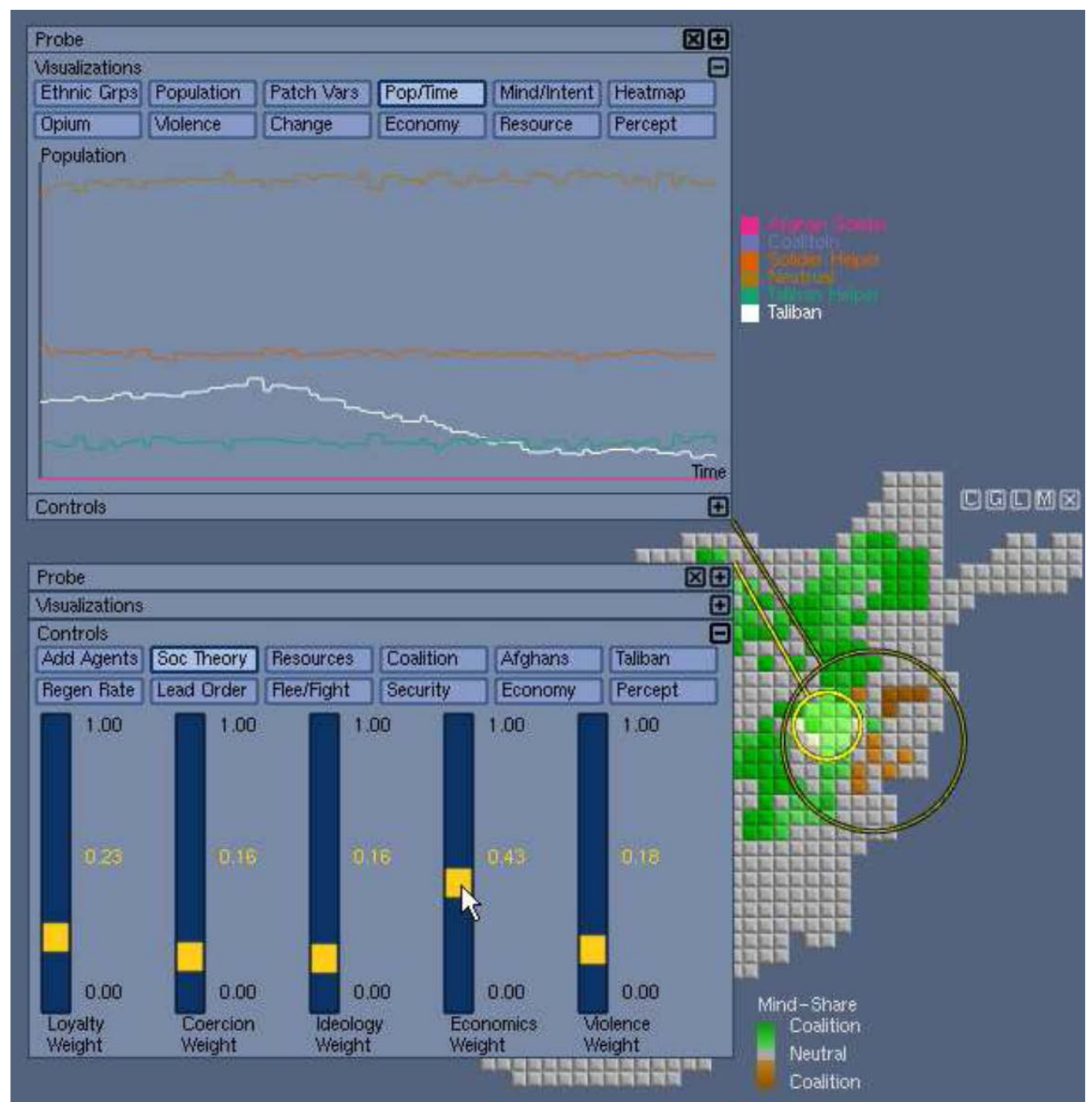


Figure A9-4. Shown here, the user has created two probes, one over a Taliban-controlled area (magenta circle/brown cells) and one over a coalition-controlled area (yellow circle/green cells). Each is set to display the relative populations of each type of agent using parallel coordinates. Then by choosing to create a direct comparison (bottom right pane) of the two probes, the user can see the values from each region-of-interest together in a single visualization.

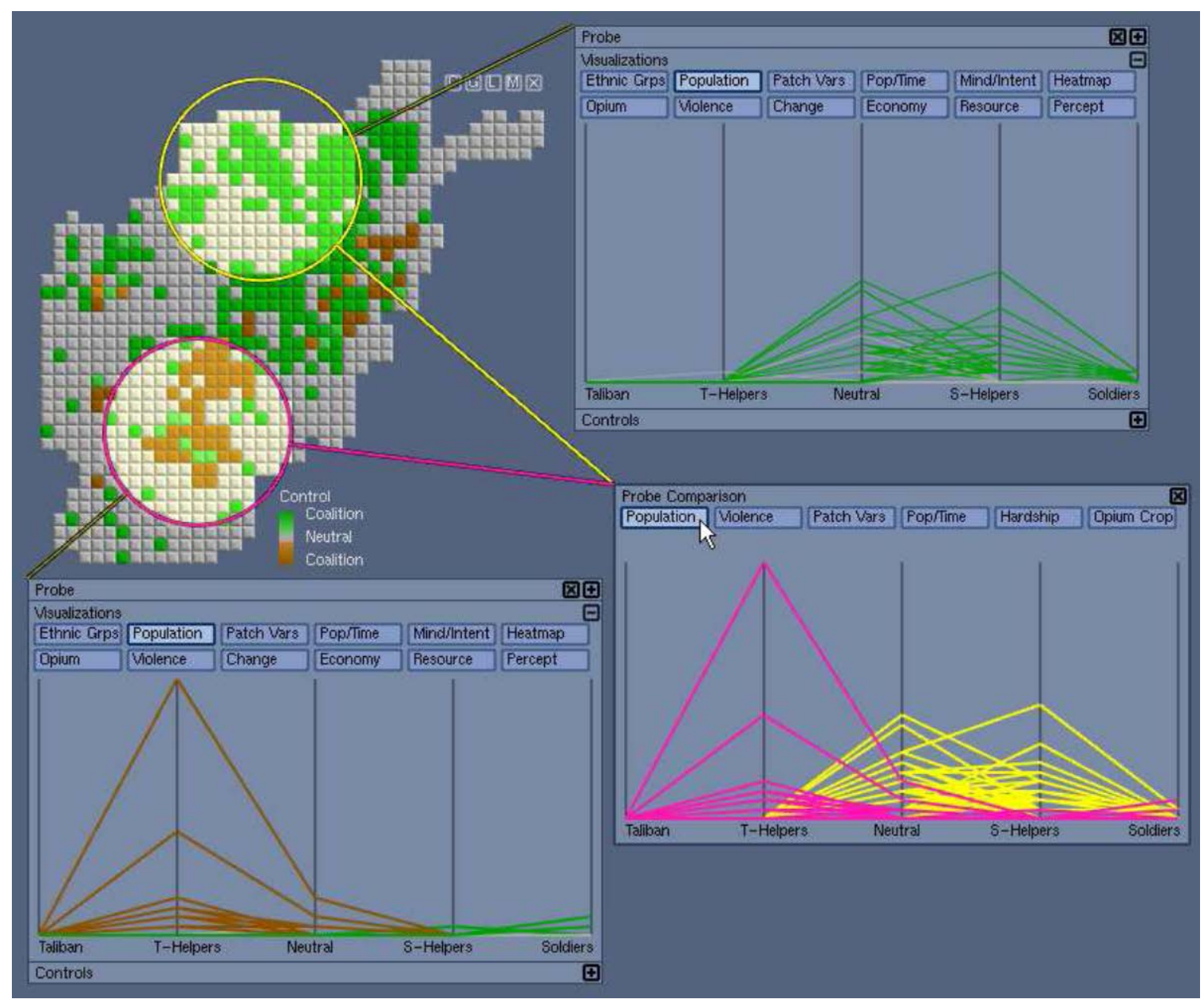

A common task when testing social theories is to directly compare two regions-ofinterest. With the probe interface, this task is again trivial and obvious. As shown in Figure A9-4, a comparison pane can be created between two existing probes to visualize the relationships between the two regions. Numerous possible operations are possible within this framework. In this example, the user has selected a "union” operation, combining the two selected regions into a single view, immediately revealing the differences in population characteristics.

The most obvious benefit gained by adding probes to this application is the ability to inspect multiple local regions at once. Whereas the original fixed visualizations once reflected the global model's values, we now have any number of dynamically created 
visualizations, each able to reflect the values in regions-of-interest of every size and shape imaginable. We can now provide superior comparison capabilities by directly presenting regions-of-interest together or separately in their own visualizations.

The probe interface also allows geospatial-based manipulation of the simulation and visualization. The user gains the freedom to choose regions-of-interest of any size and shape and interact with their properties directly, facilitating experimentation of complex social theories and immediate visualization of their effects. Finally, by replacing the static interface with an unrestrained workspace, enabling and encouraging the user to add and remove "on-demand" interface elements as needed, we not only remove the original clutter and wasted screen space, but also extend the single-user application into one that has potential to support multiple users. 


\section{Appendix 10 - Visual Plots of NetLogo and ORMAC Comparisons}

Figure A10-1. Time-series plots of each run, outputs from both NetLogo and ORMAC simulations

(a) 3 days/tick, legitimacy theory

Results for RUN 1

Pro-Taliban agents

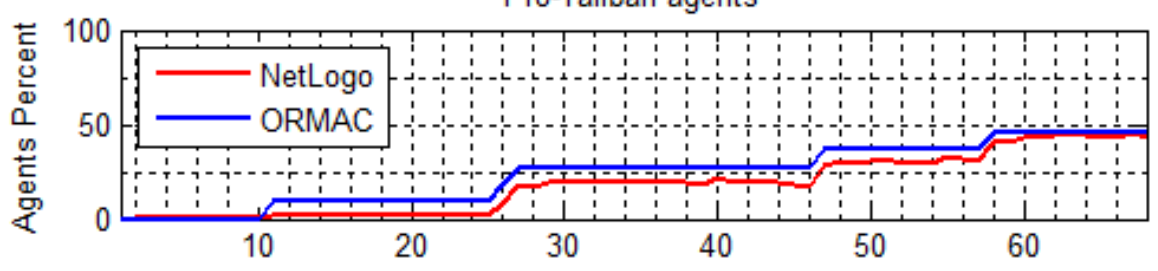

Neutral agents

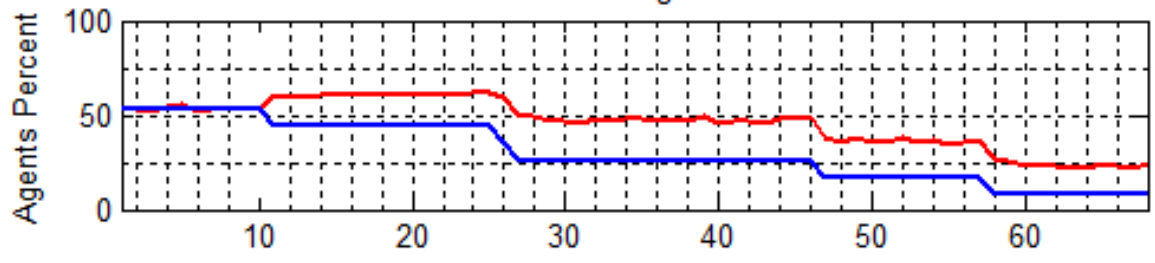

Pro-Govt. agents

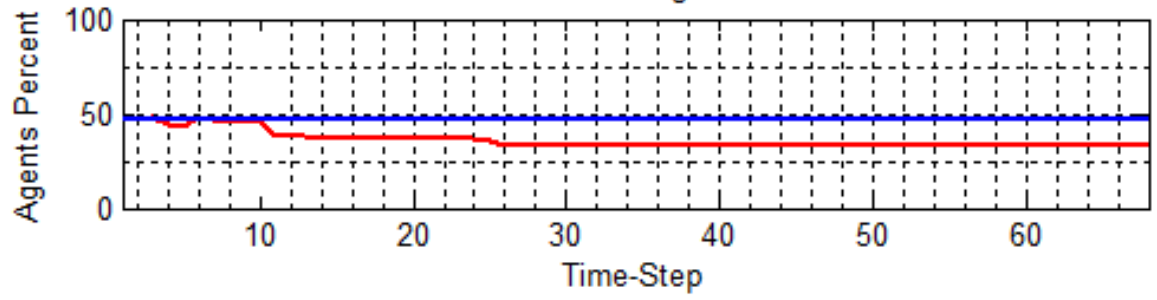


(b) 3 days/tick, representative theory
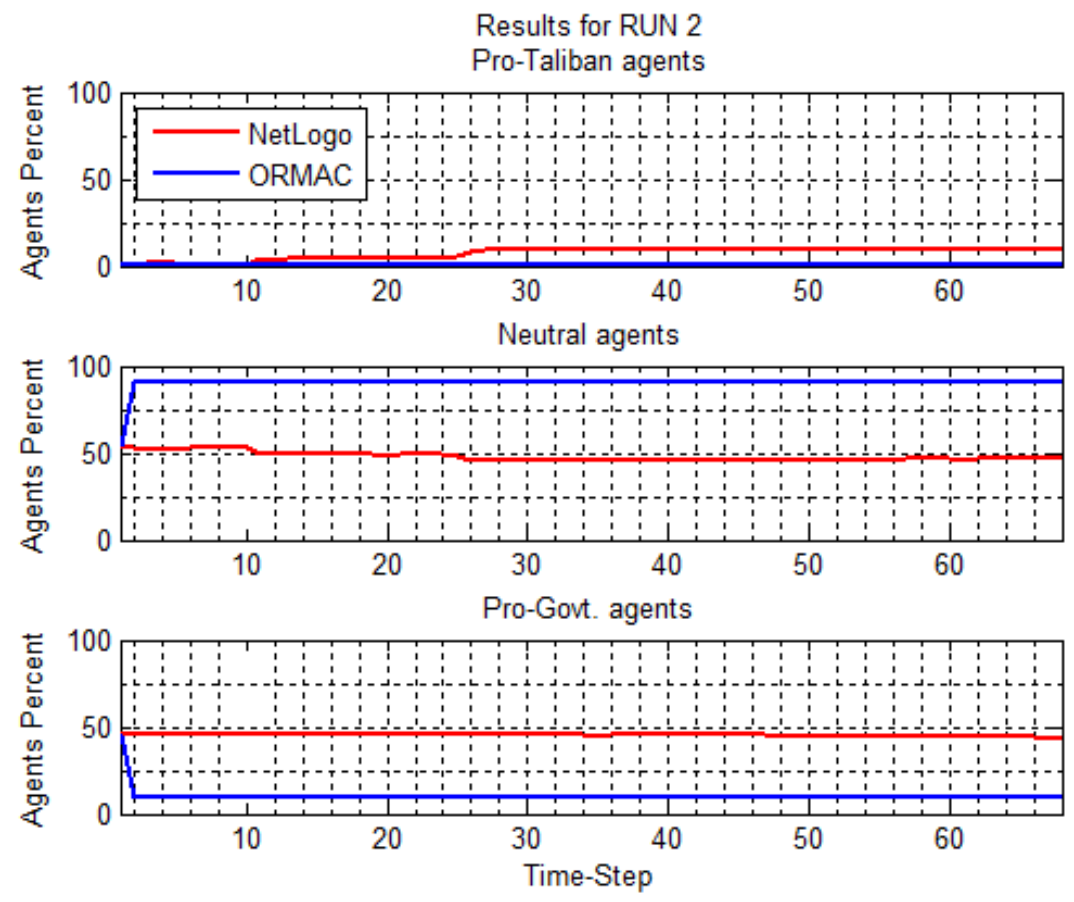

(c) 3 days/tick, coercion theory
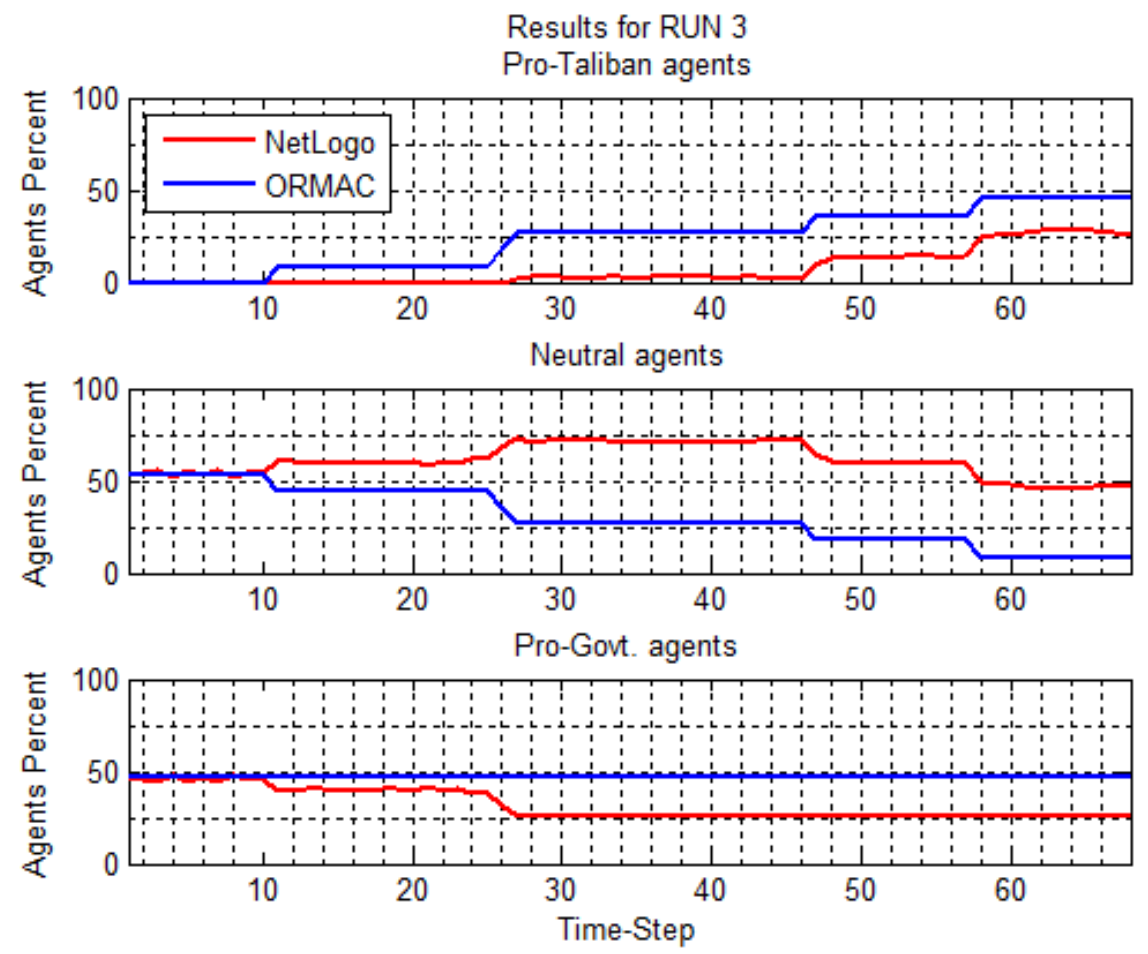
(d) 7 days/tick, legitimacy theory
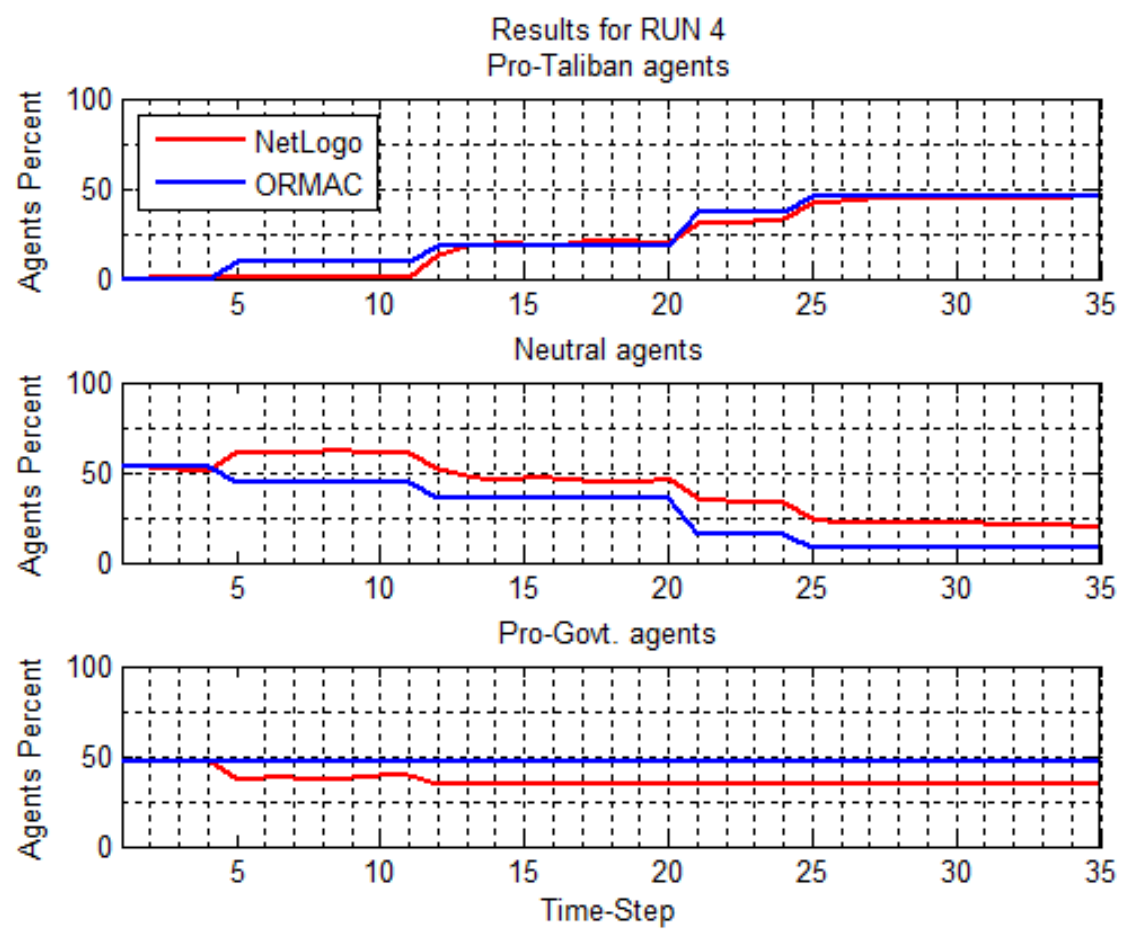

(e) 7 days/tick, representative theory
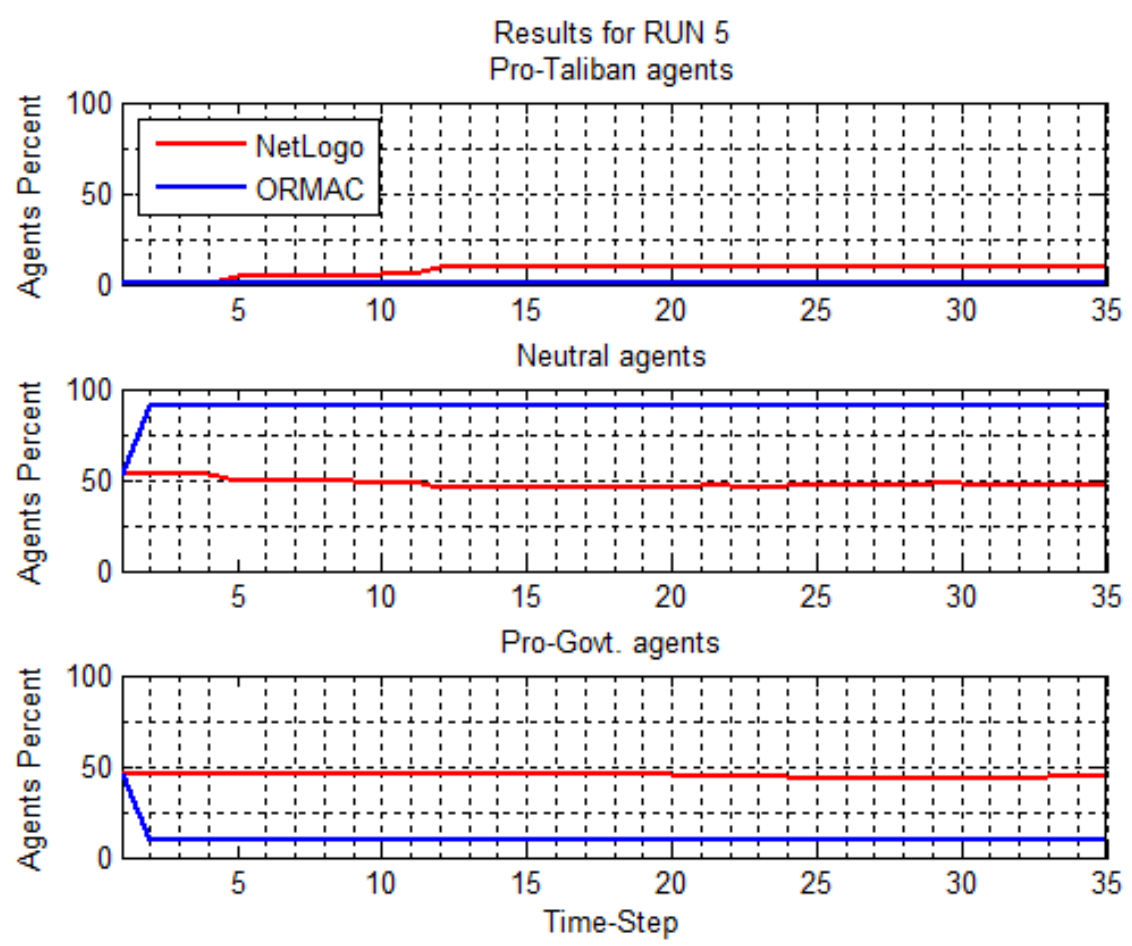
(f) 7 days/tick, coercion theory
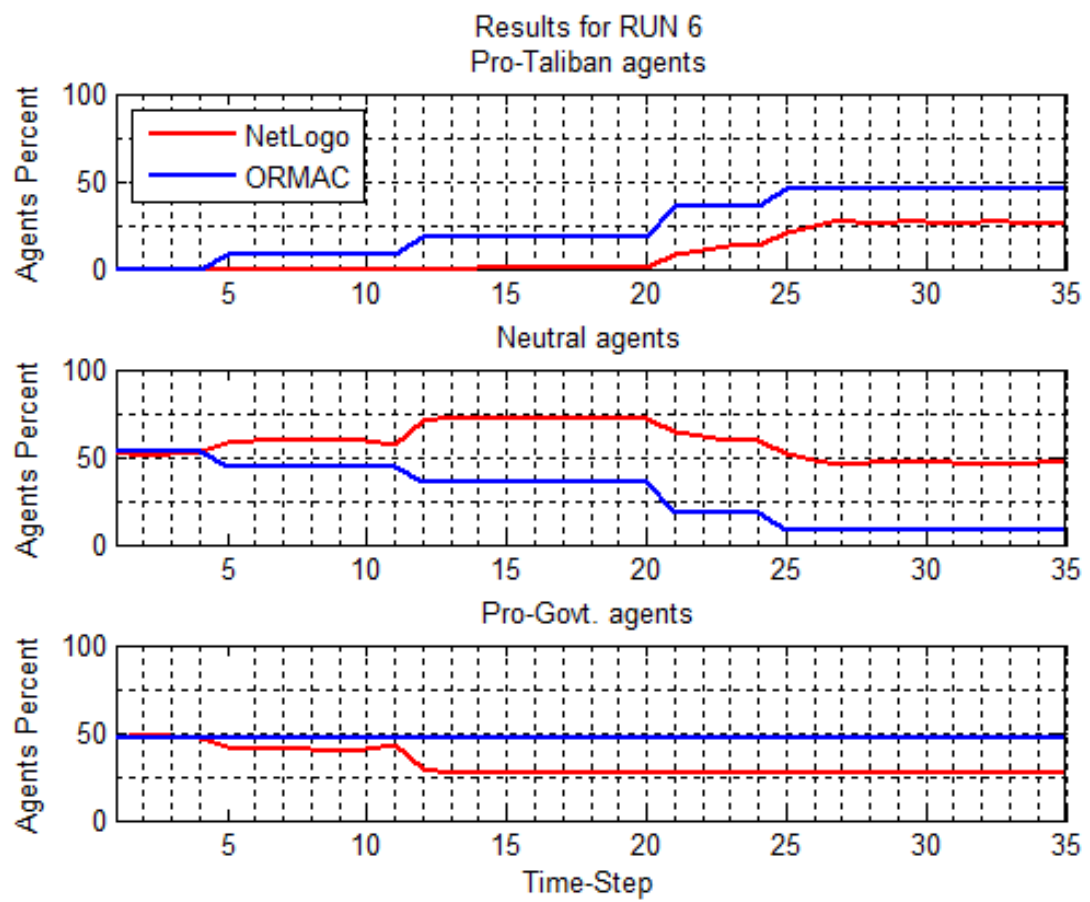

(g) 14 days/tick, legitimacy theory
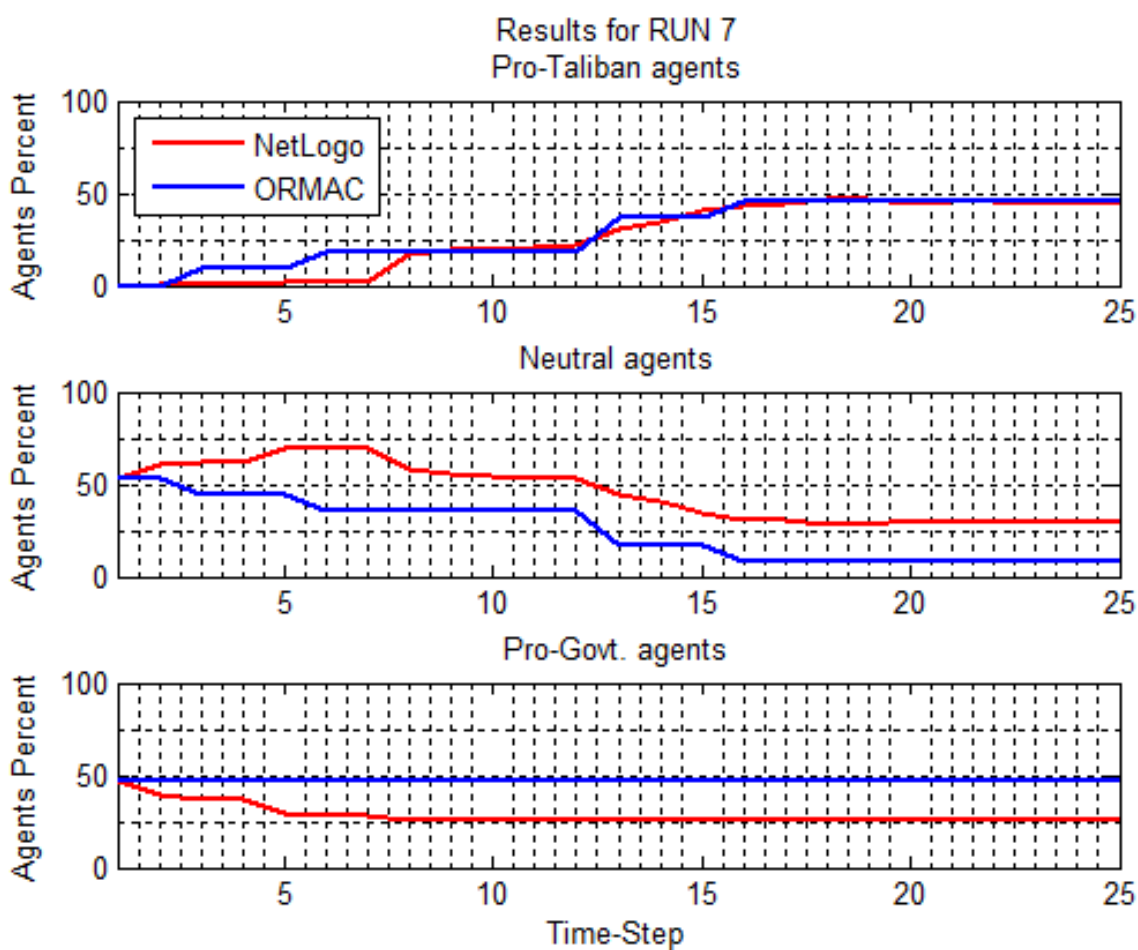
(h) 14 days/tick, representative theory
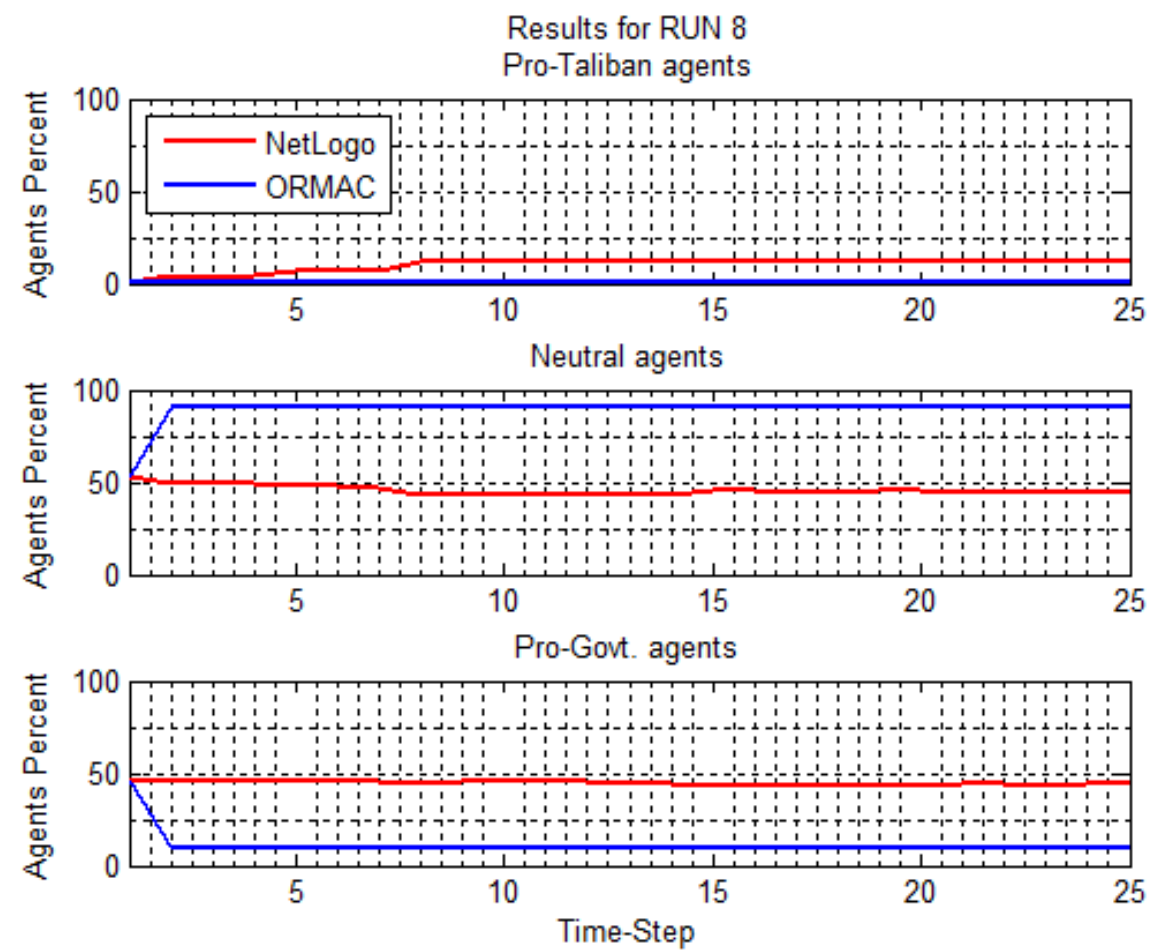

(i) 14 days/tick, coercion theory

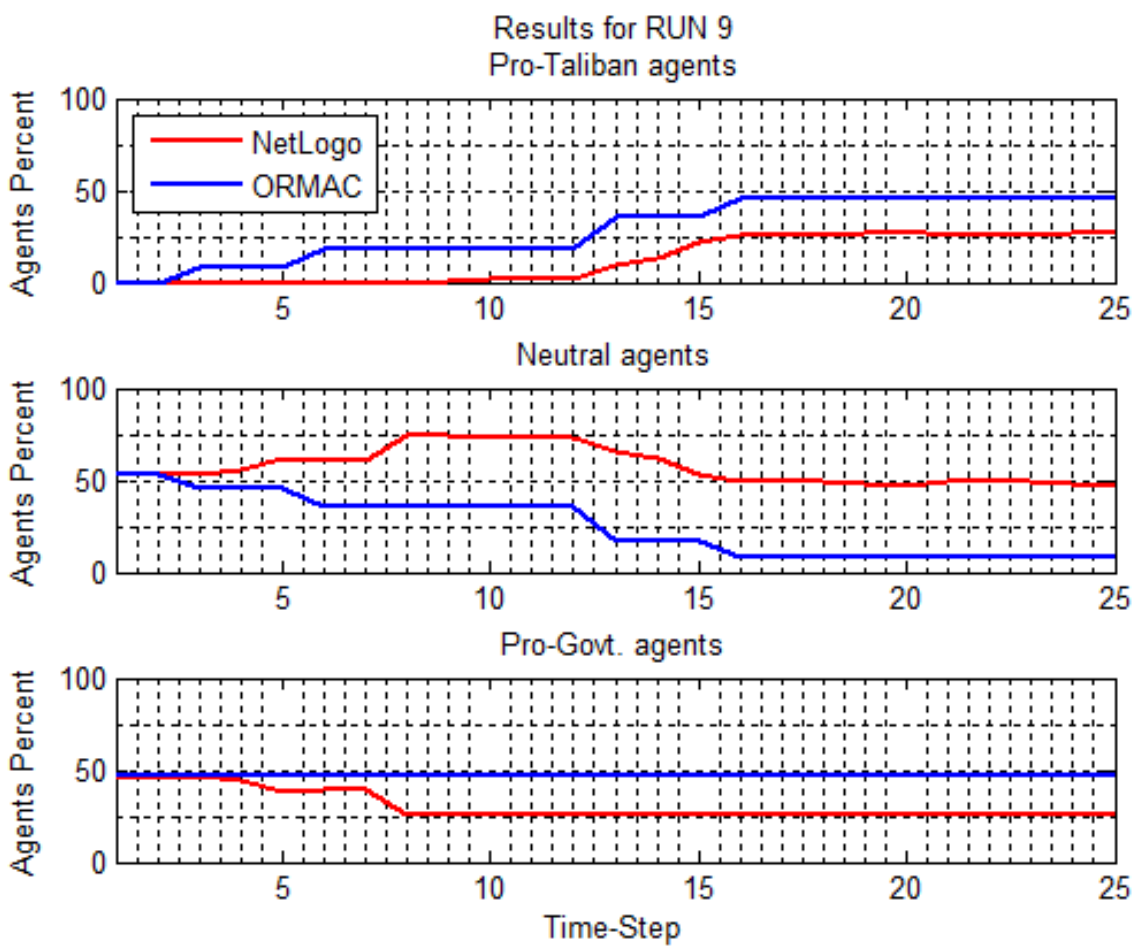

